\title{
Untersuchung von akustischen Strömungen im kHz- und GHz-Bereich
}

\author{
Dissertation \\ zur Erlangung des mathematisch-naturwissenschaftlichen Doktorgrades \\ "Doctor rerum naturalium" \\ der Georg-August-Universität Göttingen \\ im Promotionsprogramm ProPhys \\ der Georg-August University School of Science (GAUSS)
}

vorgelegt von

TILL NOWAK

geboren in

Preetz

Göttingen 2013 
$\underline{\text { Betreuungsausschuss }}$

Prof. Dr. Christoph F. Schmidt,

Drittes Physikalisches Institut, Universität Göttingen

Dr. Robert Mettin,

Drittes Physikalisches Institut, Universität Göttingen

$\underline{\text { Mitglieder der Prüfungskommission }}$

Referent: Prof. Dr. Christoph F. Schmidt,

Drittes Physikalisches Institut, Universität Göttingen

Korreferent: Prof. Dr. Werner Lauterborn,

Drittes Physikalisches Institut, Universität Göttingen

Weitere Mitglieder der Prüfungskommission:

Prof. Dr. Ulrich Parlitz,

Biomedical Physics Group, MPIDS Göttingen

Prof. Dr. Martin Rein,

Institut für Aerodynamik und Strömungstechnik, DLR Göttingen

Prof. Dr. Jörg Enderlein,

Drittes Physikalisches Institut, Universität Göttingen

Prof. Dr. Stefan Luther,

Biomedical Physics Group, MPIDS Göttingen

Tag der mündlichen Prüfung: $\quad$ 23.01.2014 




\section{Inhaltsverzeichnis}

$\begin{array}{ll}\text { 1. Einleitung } & 1\end{array}$

2. Grundlagen 5

2.1. Akustische Strömungen . . . . . . . . . . . . . 5

2.1.1. Mathematische Beschreibung .......... . 5

2.1.2. Näherung nach Eckart . . . . . . . . . . . . . 8

2.1.3. Akustische Strömungen bei sehr hoher Dämpfung . . . . . 9

2.2. Kavitation . . . . . . . . . . . . . . . . . . 10

2.2.1. Blake-Schwelle . . . . . . . . . . . . . . . . . . . 12

2.2.2. Bjerknes-Kräfte . . . . . . . . . . . . . . . . . . 13

2.2.3. Virtuelle Masse . . . . . . . . . . . . . . . . . . . . . . . . 18

2.2.4. Reibungskraft . . . . . . . . . . . . . . 20

2.2.5. Oberflächeninstabilitäten . . . . . . . . . . . . 21

2.2.6. Simulation der Blasendynamik . . . . . . . . . . . . 22

2.2.7. Blasentranslation . . . . . . . . . . . . . . . . 24

2.3. Einfluss von Gasblasen auf akustische Strömungen . . . . . . . . . 25

2.3.1. Schalldämpfung durch Blasen . . . . . . . . . . . 26

2.3.2. Strömungen im Kavitationsblasenfeld . . . . . . . . . . . . 28

3. Experimenteller Aufbau 31

3.1. Akustische Strömungen im Kavitationsblasenfeld . . . . . . . . . 31

3.1.1. Fluoreszenzmikroskop . . . . . . . . . . . . . 31

3.1.2. Wasserversorgung . . . . . . . . . . . . . 35

3.1.3. Sonotrode ................. . . 37

3.1.4. Küvette . . . . . . . . . . . . . . . . . . 38

3.2. Akustische Strömungen im Gigahertzbereich . . . . . . . . . . . . 40

3.2.1. Gigahertz-Schallwandler . . . . . . . . . . . . . . . . . 41

3.2.2. Elektrochemische Messungen . . . . . . . . . . . . . 44 
4. Untersuchung von Strömungen mit und ohne Kavitation 47

4.1. Kalibrationsmessungen und Methodik . . . . . . . . . . . . . . . . 47

4.1.1. Positionskalibration . . . . . . . . . . . . . . . 48

4.1.2. Rohdatenverarbeitung . . . . . . . . . . . . . . . . 49

4.1.3. Kalibrationsmessungen der Auslenkung . . . . . . . . . . . 50

4.2. Vergleich der Kavitationsblasenfelder bei Parametervariation . . . 55

4.2.1. Verschiedene Spannungen . . . . . . . . . . . . . 55

4.2.2. Verschiedene Temperaturen und Gasgehalte . . . . . . . 55

4.3. Strömungsfeld bei 2 bar Überdruck . . . . . . . . . . . . . . . . . 59

4.4. Strömungsfeld bei Normaldruck . . . . . . . . . . . . . . . 68

4.5. Zusammenhang zwischen Kavitations- und Strömungsfeld . . . . . 79

4.6. Energieabsorption in Abhängigkeit von Temperatur und Gasgehalt 84

4.6.1. Vergleich der Energien . . . . . . . . . . . . . . . . 90

4.7. Charakterisierung der Strömung . . . . . . . . . . . . . . . 100

4.7.1. Zeitliche Korrelation der Strömung . . . . . . . . . . . . . 101

4.7.2. Reynolds-Zahlen mit und ohne Kavitation . . . . . . . . . 103

4.7.3. Frequenzspektren . . . . . . . . . . . . 106

4.8. Detailaufgelöste Aufnahmen . . . . . . . . . . . . . . . . 108

4.8.1. Analyse von Blasenschwingung und Strömung . . . . . . . 110

5. FEM-Simulation von Schallfeld und Strömung 117

6. Diskussion der Ergebnisse mit und ohne Kavitation 121

6.1. Strömungsfelder mit und ohne Kavitation . . . . . . . . . . . . . 121

6.2. Zusammenhang zwischen Kavitations- und Strömungsfeld . . . . . 122

6.3. Zunahme der kinetischen Energiedichte mit der Spannung . . . . 123

6.4. Turbulenzmaße Korrelation und Reynolds-Zahl . . . . . . . . . . . 125

6.5. Vergleich der Energien . . . . . . . . . . . . . . . . . . 126

6.6. Detailaufgelöste Aufnahmen . . . . . . . . . . . . . . . . . 127

6.7. Einfluss des Schallfeldes auf die Strömung . . . . . . . . . . . . 128

7. Untersuchung von Strömungen im Gigahertzbereich 129

7.1. Messungen und Methodik . . . . . . . . . . . . . . . . . 129

7.1.1. PIV-Messungen und Tintenfront-Verfolgung . . . . . . . . 129

7.1.2. Elektrochemische Messungen . . . . . . . . . . . . . 130

7.2. Akustische Strömungen an einem Array-Schallwandler . . . . . . . 131

7.2.1. Großräumige Strömungen in der Küvette . . . . . . . . . . 132

7.2.2. Wirbel an der Wandleroberfläche . . . . . . . . . . . . . 133

7.2.3. Elektrochemische Messung . . . . . . . . . . . . . . . . 137 
7.3. Akustische Strömungen an einem Zwillings-Schallwandler . . . . . 141

7.3.1. Elektrische Pulsformen und Leistung . . . . . . . . . . . . . 142

7.3.2. Gepulste Strömung in der Küvette . . . . . . . . . . . . . 144

7.3.3. Strömungen bei verschiedenen Leistungen . . . . . . . . . 149

7.3.4. Elektrochemische Messung . . . . . . . . . . . . . 153

8. FEM-Simulation von Schallfeld und Strömung 155

8.1. Akustische Simulationen mit Comsol . . . . . . . . . . . . 155

8.1.1. Schallfelder und Strömungen bei $400 \mathrm{MHz}$. . . . . . . . . 155

8.1.2. Schallfelder und Strömungen bei $2 \mathrm{GHz}$. . . . . . . . . . . 159

8.2. Volumenkraftsimulationen mit Comsol . . . . . . . . . . . . 163

8.2.1. Array-Schallwandler . . . . . . . . . . . . . . . . . . . 164

8.2.2. Zwillings-Schallwandler . . . . . . . . . . . . 167

$\begin{array}{ll}\text { 9. Diskussion der Ergebnisse im Gigahertzbereich } & 173\end{array}$

9.1. PIV-Aufnahmen . . . . . . . . . . . . . . . . 173

9.2. Elektrochemische Messungen . . . . . . . . . . . . . . . . 175

9.3. Vergleich zwischen Simulation und Experiment . . . . . . . . . 176

$\begin{array}{lr}\text { 10.Zusammenfassung und Ausblick } & 179\end{array}$

$\begin{array}{ll}\text { A. Konstruktion der Sonotroden } & 183\end{array}$

A.1. Anpassen des Exponentialhorns . . . . . . . . . . . . . . . . . 183

A.2. Berechnung der Rückmasse . . . . . . . . . . . . . . . . . . . 185

$\begin{array}{lr}\text { Symbolverzeichnis } & 190\end{array}$

$\begin{array}{ll}\text { Literaturverzeichnis } & 201\end{array}$ 



\section{Kapitel 1 Einleitung}

In dieser Arbeit werden akustische Strömungen mit und ohne Kavitation untersucht und die Ergebnisse vergleichend gegenüber gestellt. Akustische Strömungen [1-4] sind gleichförmige, gerichtete Strömungen, die durch Schallabsorption innerhalb von flüssigen oder gasförmigen Medien erzeugt werden. Durch Dämpfung und nichtlineare Effekte findet ein Impulsübertrag der akustischen Wechselströmung (Schallschnelle) auf das Medium statt und erzeugt eine gerichtete Nettoströmung. In vielen industriellen Anwendungen spielen akustische Strömungen eine Rolle. So führen sie in Ultraschallreinigungsanwendungen zum Transport der abgereinigten Stoffe [5-7], sorgen in chemischen Prozessen für eine bessere Durchmischung der Edukte [8] oder werden inzwischen in der Forschung in „Lab on a Chip Anwendungen" zum Pumpen von Flüssigkeiten benutzt [9].

In Flüssigkeiten kann bei hohen Schallwechseldrücken zusätzlich zur akustischen Strömung Kavitation [10-12] auftreten. Durch starken Unterdruck in der negativen Schalldruckphase reißt die Flüssigkeit auf und es entstehen dampf- oder gasgefüllte Blasen, die in der Überdruckphase kollabieren können. Innerhalb von Kavitationsblasenfeldern sind akustische Strömungen für viele Effekte der Blasentranslation und der Strukturbildung verantwortlich. Durch die Relativbewegung zwischen Flüssigkeit und Blase und deren Wechselwirkungen beeinflussen die akustischen Strömungen im Endeffekt auch die Oberflächendynamik der Blasen [13]. Umgekehrt beeinflusst das Blasenfeld durch verstärkte Schalldämpfung [14, 15] und durch die Blasendynamik, die Mikroströmungen hervorrufen kann, wiederum die Strömung der Flüssigkeit [16-19]. Die Untersuchung von Strömungen innerhalb von Blasenwolken ist komplex, da sich die Kavitationsblasen nicht nur mit unterschiedlicher Geschwindigkeit, sondern sogar entgegengesetzt der Strömung bewegen können [20]. Speziell bei niedrigen Frequenzen sind Kavitationsblasen instabil - ihre Lebensdauer beträgt unter Umständen nur wenige Schallperioden und durch die Volumenoszillation sind sie je nach Aufnahmerate nicht immer sichtbar [21]. Zudem schwankt die räumliche Dichte der Kavitationsblasen bei niedrigen Frequenzen stark. Somit können Kavitationsblasen nicht als Indikato- 
ren für die Strömung der Flüssigkeit herangezogen werden. Im Rahmen dieser Arbeit werden deshalb (Fluoreszenz-)Tracer-Partikel [22] zur Messung der Strömung eingesetzt. Diese werden mit einer bestimmten Wellenlänge angeregt (mit $532 \mathrm{~nm}$ ) und emittieren Licht einer anderen, meist höheren, Wellenlänge (etwa $590 \mathrm{~nm})$. Um den Kontrast zu erhöhen und die Partikel von den Blasen unterscheiden zu können, werden zusätzlich geeignete Filter benötigt, um das an den Blasen gestreute anregende Licht herauszufiltern. Zu diesem Zweck wurde ein spezieller Fluoreszenzmikroskopaufbau entwickelt, mit dem es möglich ist, die Blasenbewegung, die Blasenoszillation und die Strömung simultan und zeitaufgelöst aufzunehmen. Dieser Aufbau nutzt einen kontinuierlich arbeitenden frequenzverdoppelten Neodym-YAG-Laser (Wellenlänge $532 \mathrm{~nm}$ ) zum Anregen der Partikel und eine gepulste LED gleicher Wellenlänge zur Hintergrundbeleuchtung, um die Kavitationsblasen sichtbar zu machen. Um nur die Partikel einer Ebene, senkrecht zur Beobachtungsrichtung, sehen zu können, wird der Laserstrahl mit spezieller Optik zu einem sogenannten Lichtschnitt aufgeweitet. Beide Bilder werden mit Hilfe von zwei Kameras synchron, aber leicht gegeneinander verzögert, aufgenommen.

In Kapitel 4 werden Untersuchungen der Strömungen an einer Sonotrode bei etwa $17 \mathrm{kHz}$ bei verschiedenen Temperaturen und Gasgehalten gezeigt. Um die durch die Blasen dissipierte Energie abschätzen zu können, kann die Kavitation durch Anlegen eines Überdrucks unterdrückt werden. So können die Strömungen bei gleichem Schalldruck mit und ohne Kavitation verglichen werden. Die Auswertung der Partikelbewegung erfolgt mit Hilfe von Particle Image Velocimetry (PIV) [23]. Bei konventionellen PIV-Techniken wird meist ein Doppelpulslaser verwendet. Dieser ermöglicht eine sehr hohe Zeitauflösung, aber durch die meist geringe Wiederholrate kann die Strömung innerhalb der Blasenwolke nicht zeitaufgelöst aufgenommen werden. Damit ist es unmöglich, kleinräumige Strömungen auf einzelne Blasen zurückzuführen. Der in dieser Arbeit vorgestellte Aufbau ermöglicht dies bei entsprechend genauer Kalibration. Eine alternative Methode, um die Strömungen mit der räumlichen Dichte der Blasen zu korrelieren, wäre die gleichzeitige Lasertomografie [24,25]. Allerdings lassen sich auch hiermit keine einzelnen Blasen innerhalb der Blasenwolke beobachten.

Von besonderem Interesse sind akustische Strömungen hoher Geschwindigkeit. Diese lassen sich unter Anderem durch Erhöhung der Schallleistung erzeugen, was zu oft unerwünschter Kavitation führen kann. Der im Rahmen dieser Arbeit verfolgte Ansatz verwendet sehr hohe Frequenzen, die ebenfalls zu hohen Strömungsgeschwindigkeiten führen. Mit hohen Frequenzen nimmt die Dämpfung des Schalls zu. Damit wird ein höherer Anteil des Schallimpulses in eine gleichgerichtete, schnellere Strömung überführt. Außerdem nimmt mit zuneh- 
mender Frequenz, und damit Dämpfung, die Reichweite des Schalls ab. Eine dem Wandler gegenüberliegende Grenzfläche verursacht dann schon bei geringen Abständen keine Stehwellen mehr. Der Antrieb der Strömung wird unabhängig vom Abstand des Wandlers von einer gegenüberliegenden Grenzfläche. Lediglich die Größe der sich ausbildenden Wirbel wird noch durch den Abstand beeinflusst. Bei akustischen Strömungen bei niedrigen Frequenzen ist die durch den dissipierten Schall entstehende Kraft auf die Flüssigkeit weitgehend unabhängig vom Abstand (Eckart-Strömung [1]). Beim Übergang zu hohen Frequenzen wird die Strömung dagegen nicht mehr weiträumig und fern vom Wandler angetrieben, sondern der Schall wird innerhalb einer sehr kurzen Entfernung komplett dissipiert. Dadurch findet der gesamte Impulsübertrag ebenfalls in einem sehr kleinen Volumen statt („Lighthill“-Strömung [4]). Eine Strömung außerhalb der Reichweite des Schalls wird dennoch durch den dann vorhandenen Flüssigkeitsimpuls angetrieben. Um diese Strömungen zu erzeugen, werden im Rahmen dieser Arbeit neu entwickelte, bei bis zu $2 \mathrm{GHz}$ arbeitende Dickenschwinger verwendet. Mit diesen Wandlern können sehr schnelle „Jet“-Strömungen erzeugt werden, für die es vielfältige Anwendungsmöglichkeiten gibt. So können Strömungen in sehr schmalen Spalten erzeugt [26] und so diffusionslimitierte Prozesse wie elektrochemische Beschichtung [27, 28] und chemische Reaktionen beschleunigt werden. Außerdem könnten Wandler mit sehr hohen Frequenzen nach weiterer Optimierung zum Abreinigen von Partikeln innerhalb sehr kleiner Strukturen eingesetzt werden [29]. Die meisten bisherigen Anwendungen sehr hoher Frequenzen nutzen Oberflächenwellen, sogenannte „Surface Acoustic Waves“ (SAW), zum Erzeugen von akustischen Strömungen. Diese werden in „Lab on a Chip“-Anwendungen [30] zum Transport von Flüssigkeiten [31-34], zum Beeinflussen von Partikeln [35-37] und zum Mischen $[38,39]$ benutzt. Bei Frequenzen im Gigahertzbereich wurden bisher nur wenige Versuche durchgeführt [29, 40,41].

In Kapitel 7 werden erste Untersuchungen solcher vom Flüssigkeitsimpuls getriebenen Strömungen gezeigt und es werden verschiedene Wandler verglichen. Um die Strömungen zu untersuchen, wurden Particle Shadow Velocimetry (PSV) [42], Tintenfront-Verfolgung und Chronoamperometriemessungen [43] durchgeführt. $\mathrm{Zu}$ beiden Frequenzbereichen wurden außerdem Finite Elemente Simulationen (FEM) mit Comsol [44] durchgeführt. Die Simulationen im GHz-Bereich wurden sowohl akustisch, als auch vereinfachend als reine Volumenkraftsimulationen durchgeführt. Hierbei wurde die Simulation an die beobachtete Strömung angepasst, um die erreichte Volumenkraft, und somit den Schalldruck der Schallwandler, abschätzen zu können. Die Ergebnisse werden in den Kapiteln 5 und 8 gezeigt. 



\section{Kapitel 2 Grundlagen}

\subsection{Akustische Strömungen}

Wird Schall in eine Flüssigkeit eingekoppelt, breitet er sich in dieser aus. Dadurch werden die Moleküle zu translatorischen Schwingungen angeregt. Die Geschwindigkeit dieser schwingungsförmigen Bewegung wird Schallschnelle genannt. Zusätzlich zur Bewegung durch die Schallschnelle kann durch nichtlineare Effekte und Dämpfung des Schalls ein Impulsübertrag von der oszillatorischen Bewegung der Moleküle auf eine gleichförmige Strömung stattfinden. Außerdem können aufgrund von erhöhter Dämpfung durch Kavitationsblasen sekundäre Strömungen, wie zusätzliche Nettoströmungen, und durch die Blasendynamik Verwirbelungen auftreten. In den folgenden Kapiteln soll auf einige dieser Effekte näher eingegangen werden. Hierbei werden $(\dot{)})=\mathrm{d} / \mathrm{d} t$ gleichbedeutend benutzt und bedeuten die Ableitung nach der Zeit.

\subsubsection{Mathematische Beschreibung}

In diesem Kapitel soll die aus einer Volumenkraft resultierende akustische Gleichströmung aus den hydrodynamischen Grundgleichungen abgeleitet werden. Diese Herleitung orientiert sich größtenteils an der Argumentation von NyBORG [2]. Für ein bewegtes Fluid gilt für die Kraft, die auf ein Volumenelement ausgeübt wird, die Navier-Stokes-Gleichung:

$$
\vec{f}=\rho\left[\frac{\partial \vec{u}}{\partial t}+(\vec{u} \cdot \vec{\nabla}) \vec{u}\right]=-\vec{\nabla} p+\left[\mu^{\prime}+(4 / 3) \mu\right] \vec{\nabla} \vec{\nabla} \cdot \vec{u}-\mu \vec{\nabla} \times \vec{\nabla} \times \vec{u} .
$$

Hierbei sind $p(x, y, z, t)$ der Druck, $\rho(x, y, z, t)$ die Dichte und $\vec{u}(x, y, z, t)$ die Geschwindigkeit des Fluidelements. $\mu$ ist die Scher- und $\mu^{\prime}$ die Volumenviskosität. Die Volumenviskosität spielt bei der Schallausbreitung (und Stoßwellenausbreitung) in Fluiden eine Rolle und beschreibt Relaxationsphänomene der Flüssigkeit. 
Mithilfe der Kontinuitätsgleichung

$$
\frac{\partial \rho}{\partial t}+\vec{\nabla} \cdot \vec{u}=0
$$

folgt aus Gleichung (2.1) die allgemeine Navier-Stokes-Gleichung ohne Vernachlässigung der Kompressibilität $\mathrm{d} \rho / \mathrm{d} t$ :

$$
\frac{\partial(\rho \vec{u})}{\partial t}-\vec{F}^{\prime}=-\vec{\nabla} p+\left[\mu^{\prime}+(4 / 3) \mu\right] \vec{\nabla} \vec{\nabla} \cdot \vec{u}-\mu \vec{\nabla} \times \vec{\nabla} \times \vec{u} .
$$

Hierbei ist

$$
-\vec{F}^{\prime}=\rho(\vec{u} \cdot \vec{\nabla}) \vec{u}+\vec{u} \vec{\nabla} \cdot \rho \vec{u}
$$

die durch den Schall auf ein Flüssigkeitselement wirkende Kraft.

Druck, Dichte und Geschwindigkeit lassen sich nun in einen statischen Anteil $\left(p_{0}, \rho_{0}\right.$, das Fluid wird zu Beginn als ruhend angenommen), unter Annahme einer sinusförmigen Schallwelle in einen periodisch mit der Zeit fluktuierenden Anteil $\left(p_{1}, \rho_{1}, \vec{u}_{1}\right)$ und Anteile höherer Ordnung zerlegen:

$$
\begin{aligned}
p-p_{0} & =p_{1}+p_{2}+\ldots \\
\rho-\rho_{0} & =\rho_{1}+\rho_{2}+\ldots \\
\vec{u} & =\vec{u}_{1}+\vec{u}_{2}+\ldots .
\end{aligned}
$$

Die hier gesuchten zeitunabhängigen Größen für die Beschreibung der akustischen Strömungen sind die Terme zweiter Ordnung $p_{2}, \rho_{2}$ und $\vec{u}_{2}$. Um die Näherung zweiter Ordnung zu erhalten, wird zunächst eine Lösung erster Ordnung gesucht. Unter der Annahme, dass die Schallschwingung, also die zeitliche Veränderung der Terme erster Ordnung, sinusförmig erfolgt, lassen diese sich als Realteil einer komplexen Zahl mit der Zeitabhängigkeit $\mathrm{e}^{\mathrm{i} \omega t}$ schreiben. Hierbei sind $\omega$ die Kreisfrequenz und $t$ die Zeit. Mit Hilfe der akustischen Näherung

$$
p_{1}=c^{2} \rho_{1} \text {, mit der Schallgeschwindigkeit } c,
$$

folgt aus Gleichung (2.1) für die Näherung erster Ordnung für die Kraft $\vec{f}$ :

$$
\overrightarrow{f_{1}}=-c^{2} \vec{\nabla} \rho_{1}+\left[\mu^{\prime}+(4 / 3) \mu\right] \vec{\nabla} \vec{\nabla} \cdot \vec{u}_{1}-\mu \vec{\nabla} \times \vec{\nabla} \times \vec{u}_{1} .
$$

Unter der Annahme, dass die Dichteänderung durch die Schallwelle sehr gering ist, lässt sich aus Gleichung (2.2) folgern:

$$
\dot{\rho}_{1}=-\rho_{0} \vec{\nabla} \cdot \vec{u}_{1} .
$$

Mit derselben Annahme lässt sich nun mit Hilfe der Gleichungen (2.3) und (2.6) eine von $p_{1}$ und $\rho_{1}$ unabhängige Gleichung für $\vec{u}_{1}$ aufstellen:

$$
\left[2 k^{-2}+\mathrm{i} b \beta^{-2}\right] \vec{\nabla} \vec{\nabla} \cdot \vec{u}_{1}+2 \vec{u}_{1}=\mathrm{i} \beta^{-2} \vec{\nabla} \times \vec{\nabla} \times \vec{u}_{1} .
$$


Durch die obigen Annahmen ergeben sich $k, \beta$ und $\mu b$ als Konstanten zu:

$$
k=\frac{\omega}{c} ; \quad \beta^{2}=\frac{\omega \rho}{2 \mu} ; \quad \mu b=\left[\mu^{\prime}+(4 / 3) \mu\right] .
$$

Zusammen mit der Wellenzahl $k=k_{r}-\mathrm{i} \alpha_{0}$ (Dispersionsrelation) ergibt sich der Dämpfungskoeffizient zu:

$$
\alpha_{0}=\frac{\left((4 / 3) \mu+\mu^{\prime}\right) \omega^{2}}{2 \rho_{0} c^{3}} .
$$

Die Amplitude einer sich in einem Medium in $z$-Richtung ausbreitenden Schallwelle beträgt dann $A(z)=A_{0} \mathrm{e}^{-\alpha_{0} z}$. Aus den obigen Betrachtungen ergeben sich zwei Fälle. Im ersten Fall, dass sich die Schallwelle im unbegrenzten Medium ausbreitet, ist das Schallfeld rotationsfrei und es gelten unter der Annahme, dass $\alpha_{0} \ll k$, folgende Zusammenhänge:

$$
\vec{\nabla} \times \vec{u}_{1 \mathrm{a}}=0, \quad \vec{\nabla} \vec{\nabla} \cdot \vec{u}_{1 \mathrm{a}}=\vec{\nabla}^{2} \vec{u}_{1 \mathrm{a}}=-\left(k-\mathrm{i} \alpha_{0}\right)^{2} \vec{u}_{1 \mathrm{a}} .
$$

Im zweiten Fall, nahe an Grenzflächen, ist das Schallfeld wegen der akustischen Grenzschicht, der Bedingung, dass die Schallschnelle an der Oberfläche gleich Null ist, nicht als wirbelfrei anzunehmen und es gilt:

$$
\vec{\nabla} \cdot \vec{u}_{1 \mathrm{~b}}=0, \quad-\vec{\nabla} \times \vec{\nabla} \times \vec{u}_{1 \mathrm{~b}}=\vec{\nabla}^{2} \vec{u}_{1 \mathrm{~b}}=2 \mathrm{i} \beta^{2} \vec{u}_{1 \mathrm{~b}} .
$$

Die Geschwindigkeit erster Ordnung $\vec{u}_{1}$ setzt sich folglich aus einem rotationsfreien Teil $\vec{u}_{1 \mathrm{a}}$ und einem inkompressiblen Teil $\vec{u}_{1 \mathrm{~b}}$ zusammen. Der kompressionsfreie Teil spielt beim Rayleigh-Streaming [45] in stehenden Wellen und beim Schlichting-Streaming [46] in Grenzschichten eine große Rolle. Auf diese beiden Arten der akustischen Strömungen wird im Rahmen dieser Arbeit aber nicht näher eingegangen, sondern nur der wirbelfreie Fall behandelt, da es sich bei den untersuchten Strömungen um größtenteils freie Strömungen handelt.

Um die Geschwindigkeit der akustischen Strömung $\vec{u}_{2}$ zu erhalten, muss Gleichung (2.3) bis zur zweiten Ordnung entwickelt werden. Da die resultierende Geschwindigkeit zweiter Ordnung im Fall schwacher Dämpfung zeitlich konstant ist, wird eine Zeitmittelung durchgeführt. Außerdem ist der Zeitmittelwert der instationären Beschleunigung $\partial\left(\rho \vec{u}_{1}\right) / \partial t$ im eingeschwungenen Zustand Null. Es folgt für die resultierende Kraft:

$$
-\vec{F}=-\vec{\nabla} p_{2}+\left[(4 / 3) \mu+\mu^{\prime}\right] \vec{\nabla} \vec{\nabla} \cdot \vec{u}_{2}-\mu \vec{\nabla} \times \vec{\nabla} \times \vec{u}_{2},
$$

wobei sich die linke Seite der Gleichung als die aus der Geschwindigkeit erster Ordnung resultierende Kraft schreiben lässt:

$$
-\vec{F} \equiv \rho_{0}\left\langle\left(\vec{u}_{1} \cdot \vec{\nabla}\right) \vec{u}_{1}+\vec{u}_{1}\left(\vec{\nabla} \cdot \vec{u}_{1}\right)\right\rangle .
$$


Der Zeitmittelwert wird hierbei durch $\langle\ldots\rangle$ bezeichnet. Im einfachsten Fall einer sich in einem unbegrenzten Medium in $z$-Richtung ausbreitenden ebenen gedämpften Schallwelle ergibt sich für die Schallschnelle:

$$
u_{1}=A \mathrm{e}^{-\alpha_{0} z} \cos (\omega t-k z) .
$$

Hierbei ist $A$ die Schallschnelleamplitude des Schalls direkt an der Schallquelle (Schallwandler). Die wirkende Kraft ergibt sich also zu:

$$
F_{z}=-2 \rho_{0}\left\langle u_{1} \frac{\partial u_{1}}{\partial z}\right\rangle=\rho_{0} \alpha_{0} A^{2} \mathrm{e}^{-2 \alpha_{0} z}
$$

Dieser Zusammenhang mit nur einer Komponente der Volumenkraft in Richtung der Ausbreitungsrichtung der Schallwellen wurde auch für die Simulation der akustischen Strömungen im Ergebnisteil dieser Arbeit verwendet (Kapitel 8).

\subsubsection{Näherung nach Eckart}

Eine Lösung des Strömungsfeldes einer akustischen Strömung wurde von ECKART [1] gezeigt. Hierbei wird ein Kanal, beziehungsweise eine Röhre, mit dem Durchmesser $r_{0}$ angenommen (Abbildung 2.1). An einem Ende, in eine geschlossene Wand eingelassen, befindet sich der Schallwandler (Schallquelle), der einen scharf begrenzten Schallstrahl mit dem Radius $r_{1}$ abstrahlt. Das andere Ende ist für die Flüssigkeitsströmung geschlossen, lässt den Schall aber ungehindert passieren. Für eine Strömung in einer zylindrischen Röhre mit dem Durchmesser $r_{0}$ und schallabsorbierendem geschlossenen Ende folgt mit einem Radius $r_{1}<r_{0}$ des scharf begrenzten, sich in $z$-Richtung ausbeitenden Schallstrahls für die Strömungsgeschwindigkeit in $z$-Richtung:

$$
u_{2 z}= \begin{cases}U_{0}\left\{\frac{1}{2}\left(1-\frac{x^{2}}{y^{2}}\right)-\left(1-\frac{1}{2} y^{2}\right)\left(1-x^{2}\right)-\ln y\right\} & , 0 \leq x \leq y, \\ -U_{0}\left\{\left(1-\frac{1}{2} y^{2}\right)\left(1-x^{2}\right)+\ln x\right\} & , y \leq x \leq 1\end{cases}
$$

Hierbei sind $x=r / r_{0}$ die normierte Position, $y=r_{1} / r_{0}$ die normierte Grenze des Schallstrahls und

$$
U_{0}=\frac{\alpha_{0} \rho_{0} u_{1}^{2}}{\mu} r_{1}^{2}
$$

die Geschwindigkeit in der Mitte (in $r$-Richtung) des Schallstrahls. Durch das geschlossene Ende gibt es eine negative Rückströmung außerhalb des Schallstrahls (für $y \leq x \leq 1$ ) an den Rändern des Rohres. Für diese Lösung wird angenommen, dass sich der Schallstrahl trotz der Schalldämpfung nicht wesentlich abschwächt. Die Strömung wird an jedem Punkt des Schallstrahls in z-Richtung gleichstark 


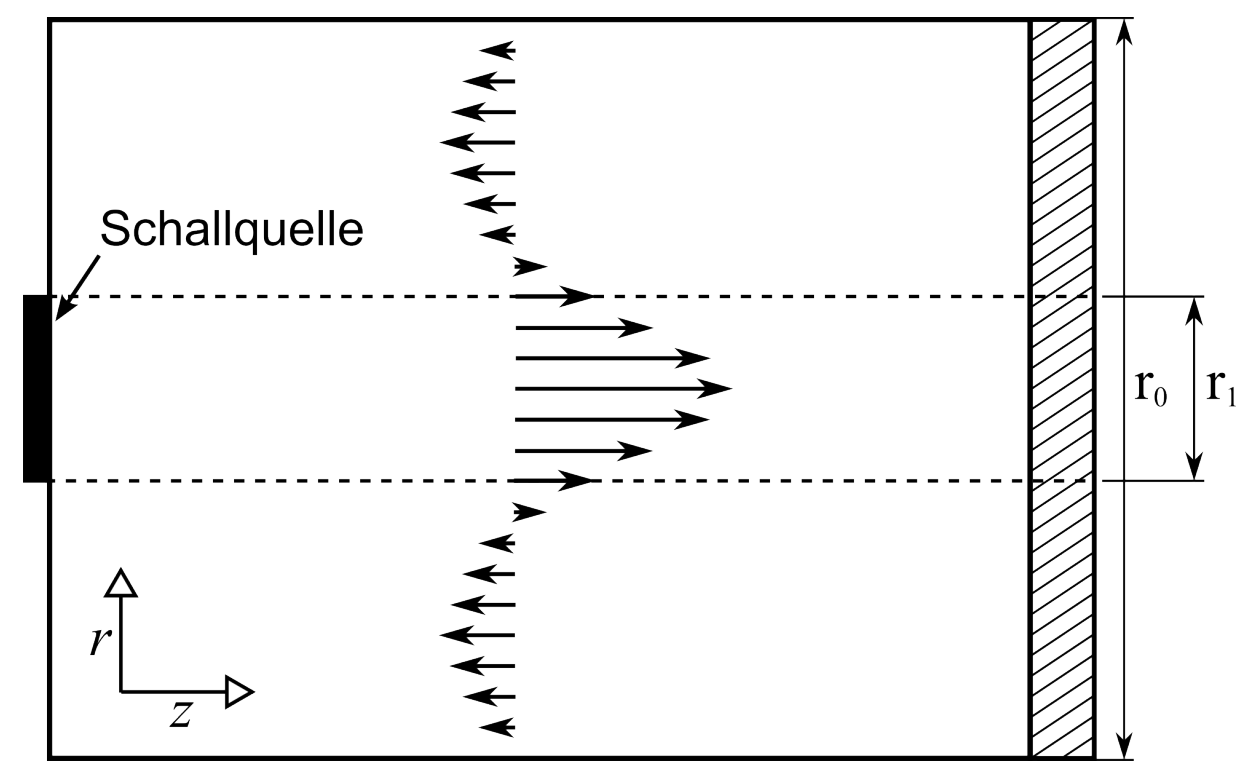

Abbildung 2.1.: Akustisches Strömungsfeld in einem geschlossenen Kanal nach ECKART (Zeichnung nach [47]).

getrieben, die Geschwindigkeit ist also unabhängig von der Entfernung. Außerdem überwiegen bei sehr langsamen Strömungen die Viskositätsterme, weshalb die Strömung zeitlich konstant bleibt und zum Stillstand kommt, sobald der Impulsübertrag durch die Schallschnelle (Impulsterm erster Ordnung) nicht mehr stattfindet. Die akustische Strömungsgeschwindigkeit $u_{2 z}$ ist proportional zur kinetischen Energiedichte des Schalls $E_{\text {kin, Schall }}=1 / 2 \rho u_{1}^{2}$, mit der Schallschnelle $u_{1}$. Die Schallschnelle ist wiederum proportional zum Schalldruck, der linear mit der am Schallwandler anliegenden elektrischen Spannung $U_{\text {el }}$ steigt. Wird die kinetische Energie der akustischen Strömung (proportional zu $u_{2 z}^{2}$ ) betrachtet, folgt diese also $U_{\mathrm{el}}^{4}$.

\subsubsection{Akustische Strömungen bei sehr hoher Dämpfung}

Für Strömungen bei sehr hohen Schallfrequenzen, oder allgemeiner bei sehr hoher Schalldämpfung, lassen sich die Vereinfachungen der klassischen Betrachtung der akustischen Strömungen nach NyBORG nicht mehr anwenden, da diese Impulseffekte innerhalb der akustischen Strömung (also die Impulsterme höherer Ordnung) vernachlässigen. Wird der Dämpfungskoeffizient $\alpha_{0}$ erhöht, bleibt die gesamte ausgeübte Volumenkraft $\left(\int \vec{F} \mathrm{~d} V\right)$ auf die Flüssigkeit konstant. Diese wird allerdings auf einer kürzeren Strecke ausgeübt, was zu einer höheren Energiedichte und somit zu höheren Geschwindigkeiten in der Nähe der Schallquelle führt. In der Lösung der akustischen Strömung nach NyBORG wurde der Impuls- 
term aufgrund seiner mathematischen Ordnung vernachlässigt. Für Strömungen bei niedrigen Reynolds-Zahlen (Reynolds-Zahlen setzen die Geschwindigkeit und eine charakteristische Länge der Strömung mit der Viskosität der Flüssigkeit in Beziehung) überwiegen bei Weitem die viskosen Terme zweiter Ordnung, weshalb diese Vereinfachung gerechtfertigt ist. Das heißt, die Strömung kommt zum Erliegen, sobald die treibende Kraft nicht mehr vorhanden ist. Wird die Strömungsgeschwindigkeit aber höher, lassen sich die Impulsterme der Strömung nicht mehr vernachlässigen, der in der Strömung vorhandene Impuls sorgt für ein Bestehen der Strömung über die Reichweite oder Dauer der treibenden Kraft hinaus. Bei hohen Dämpfungen, zum Beispiel durch hohe Schallfrequenzen, muss deshalb eine erweiterte Theorie nach Lighthill [4] angewendet werden. Die Schallschnelle und somit die kinetische Energie im Schallfeld nimmt durch die Dämpfung mit zunehmender Entfernung von der Quelle ab, es wird also immer weniger Energie auf die gleichförmige Strömung übertragen. Der durch die Schallschnelle übertragene Impuls $\left(\propto u_{1}\right)$ nimmt mit zunehmender Entfernung ab. Durch den höheren Impulsterm der Navier-Stokes-Gleichung wird aber ein zusätzlicher Impuls durch die getriebene Strömung übertragen, der dem Schallimpuls aufaddiert wird. Die resultierende Strömungsgeschwindigkeit nimmt mit der Entfernung von der Quelle zu, bis der Schall vollkommen dissipiert ist. Das heißt, die Strömung nimmt die Form eines „Jets“ an (siehe Abbildung 2.2). Ein extremes Beispiel hierfür ist die Strömung bei sehr hohen Frequenzen. Der Schall ist nach einer Entfernung $L=1 / \alpha_{0}$ um 1/e in kinetische oder thermische Energie dissipiert und die resultierende Strömung wird rein durch den Impulsübertrag der sich ausbreitenden Flüssigkeit getrieben.

\subsection{Kavitation}

Das folgende Kapitel über Kavitation ist zu großen Teilen aus meiner Diplomarbeit übernommen [20].

Aus Gründen der Konvention werden in diesem Kapitel Zeitableitungen des Blasenvolumens, des Blasenradius und der Position der Blase mit $\dot{V}, \dot{R}$ beziehungsweise $\dot{X}$ und zweite Ableitungen mit $\ddot{R}$ bezeichnet.

Kavitation bezeichnet allgemein die Bildung von Hohlräumen in einer Flüssigkeit durch mechanische Zugspannung [48]. Hierbei muss zwischen harter Kavitation, bei der die Blasen einen starken Kollaps ausführen, und weicher Kavitation, bei der die Blasen nur schwach schwingen, unterschieden werden.

Für Kavitation gibt es zwei übergeordnete Ursachen [49]. Ein Grund ist ein Energieeintrag in eine Flüssigkeit. Dies kann durch lokale Wärmezufuhr, Funkenent- 


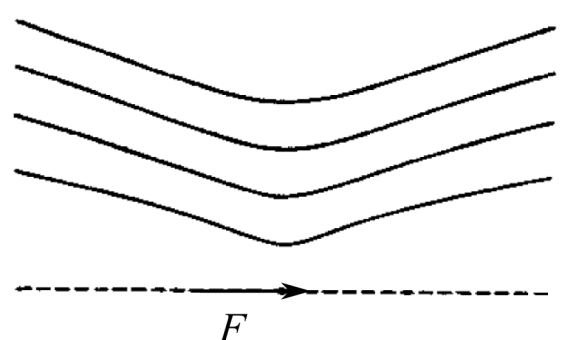

a)

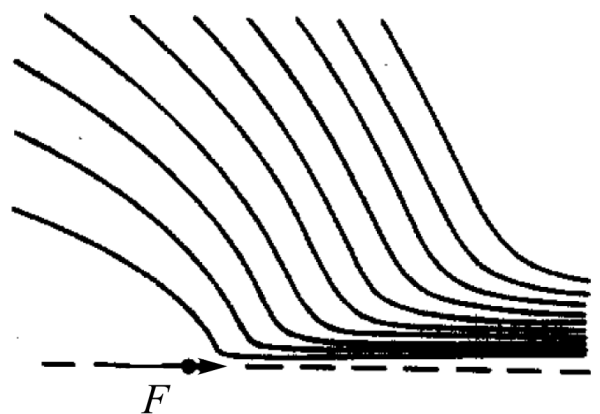

b)

Abbildung 2.2.: Strömungslinien von akustischen Strömungen beim Übergang von geringer Dämpfung bei niedrigen Frequenzen (a) zu hoher Dämpfung bei hohen Frequenzen (b). Der Volumenstrom zwischen den Strömungslinien ist konstant. Die die Strömung antreibende Kraft ist $F=c^{-1} P_{\text {akustik }}$ mit der akustischen Leistung $P_{\text {akustik. }}$. Die gestrichelte Linie repräsentiert die Symmetrieachse. Mit Zunahme der Dämpfung wird die Zuströmung unsymmetrisch und die Strömung nimmt die Form eines Jets an. (Zeichnungen entnommen aus [4])

ladung [50] oder zum Beispiel durch Laserbeschuss in einer ansonsten „kalten“ Flüssigkeit erfolgen [51,52]. Bei diesen Ursachen handelt es sich im Grunde um lokales Sieden. Sie werden im Rahmen dieser Arbeit nicht näher betrachtet, da der Fokus der Arbeit auf der zweiten Form liegt.

Eine zweite Form der Kavitation ist das Aufreißen einer Flüssigkeit unter Zugspannung. Dies kann unter anderem in an Gegenständen vorbeiströmenden Flüssigkeiten und bei in Flüssigkeiten bewegten Gegenständen auftreten. Diese Form der Kavitation in Strömungen heißt hydrodynamische Kavitation. Da sie Leistungsverluste, Geräusche und starke Beschädigungen an den umströmten Gegenständen hervorrufen kann [53], die meist unerwünscht sind, gilt es, sie in den meisten Fällen zu verhindern.

Die in dieser Arbeit untersuchte Kavitation entsteht aufgrund von starken Druckschwankungen durch eingestrahlte Schallfelder [12,54,55], in denen die Flüssigkeit in der Unterdruckphase punktuell aufreißt. Auftretende Blasen werden oft von wenigen bis zu einigen hundert Mikrometern aufgezogen, um bei Beginn der Überdruckphase schlagartig zu kollabieren. Diese akustische Kavitation wird unter anderem zur Reinigung eingesetzt [56]. Allerdings ist auch die akustisch induzierte Kavitation oft, wie zum Beispiel in einigen Fällen des diagnostischen oder therapeutischen Ultraschalls, unerwünscht, ja sogar schädlich. Ein Grund für die reinigende, aber auch zerstörerische Wirkung ist der nach der Unter- 
druckphase auftretende Kollaps der Hohlräume (Blasen). Durch die sehr hohen Wandgeschwindigkeiten und das meist sehr kleine Volumen im Kollaps entstehen während des Kollabierens sehr hohe Strömungsgeschwindigkeiten der umgebenden Flüssigkeit und sehr starke Druckwellen im Moment des kleinsten Volumens. Außerdem können die Temperaturen in kollabierenden Blasen durch die Fokussierung der Kollapsenergie und die dadurch entstehende hohe Energiedichte sehr hoch werden. Kollabiert eine Blase vor einer festen Grenzfläche, bildet sich durch die ungleichmäßige Zuströmung ein Druckgradient aus. Durch diesen oder durch andere vorhandene Druckgradienten (Schallfelder, Gravitation, Strömungen) kann die Blase zudem mit einem sogenannten Jet kollabieren. Dies ist ein schneller Flüssigkeitsstrahl, der durch die Blase hindurch in Richtung des schwächeren Drucks gerichtet ist (zum Beispiel auf feste Grenzflächen) und ebenfalls Schäden verursachen kann.

Für das Entstehen von Kavitation spielt die sogenannte Kavitationsschwelle [10] eine Rolle. Sie bezeichnet den Druck bei dem die Flüssigkeit aufreißt. Diese Kavitationsschwelle ist abhängig von verschiedenen Eigenschaften der beschallten Flüssigkeit, wie der Temperatur, dem Gasgehalt und dem Vorhandensein von Fremdkörpern. Diese Fremdkörper werden auch Kavitationskeime [57] genannt. Außerdem lässt sich die Kavitationsschwelle durch Erhöhung des statischen Drucks zu höheren Schalldrücken verschieben. Des Weiteren ist die Kavitationsschwelle von der Schallfrequenz abhängig. Je höher die Schallfrequenz, desto höher ist der benötigte Schalldruck. Im Gigahertzbereich werden je nach Temperatur und Gasgehalt einige hundert bis tausend Bar benötigt um Kavitation zu erzeugen.

\subsubsection{Blake-Schwelle}

Wenn der äußere Druck dem Innendruck einer sphärischen Blase entspricht, befindet sich diese im statischen Gleichgewicht. Hierbei entspricht der Innendruck dem Gas- oder Dampfdruck gelöster Gase beziehungsweise der jeweiligen Flüssigkeit und der äußere Druck setzt sich aus dem statischen Druck $p_{0}$ und der Oberflächenspannung $\sigma$ der Blase zusammen. Das heißt also für die Gleichgewichtsbedingung:

$$
p_{\mathrm{i}}=p_{0}+\frac{2 \sigma}{R_{\mathrm{n}}}, \text { mit dem Gleichgewichtsradius } R_{\mathrm{n}} .
$$

Im isothermen Fall gilt für eine einem konstanten Unterdruck $\left(p_{0}-p_{\mathrm{A}}\right)$ mit $\left(p_{\mathrm{A}}>0\right)$ ausgesetzte Blase:

$$
\hat{p}_{\mathrm{i}}=p_{\mathrm{i}}=\left(\frac{R_{\mathrm{n}}}{\hat{R}_{\mathrm{n}}}\right)^{3}=p_{0}-p_{\mathrm{A}}+\frac{2 \sigma}{\hat{R}_{\mathrm{n}}} .
$$


Hierbei ist $\hat{p}_{\mathrm{i}}$ der nach $p_{\mathrm{i}} V_{\mathrm{i}}=\hat{p}_{\mathrm{i}} \hat{V}_{\mathrm{i}}$ reduzierte Innendruck und $\hat{R}_{\mathrm{n}}$ der neue Gleichgewichtsradius. Wird die Gleichung nach $\hat{R}_{\mathrm{n}}$ aufgelöst, lässt sich feststellen, dass ab einem Schwellenwert von $p_{\mathrm{A}}$ keine Gleichgewichtslösung mehr existiert: Die Blase expandiert dann ohne Grenzen. Für eine Blase mit $R_{\mathrm{n}}=R_{\mathrm{B}}$ gilt für diese Schwelle (Blake-Schwelle):

$$
P_{\mathrm{B}}=p_{0}+\frac{8 \sigma}{9}\left[\frac{3 \sigma}{2\left[p_{0}+\left(2 \sigma / R_{\mathrm{B}}\right)\right] R_{\mathrm{B}}^{3}}\right]^{1 / 2} .
$$

Wird die Gleichung umgekehrt für festes $P_{\mathrm{B}}$ gelesen, lässt sich ein kritischer Radius $R_{\mathrm{B}}$ finden, der die Schwelle für unbegrenztes Blasenwachstum anzeigt. Diese Schwelle heißt auch (statische) Blake-Schwelle [10]. Dieses Phänomen tritt ebenfalls bei periodisch getriebenen Blasen zu Tage. Hier schwingen bei vorgegebener Anregungsfrequenz und -amplitude die Blasen oberhalb eines bestimmten Ruheradius stark auf (mit anschließendem Kollaps, da sie mit Wechseldruck angeregt werden). Kleinere Blasen schwingen dagegen nur schwach um ihren Ruheradius. Diese sogenannte dynamische Blake-Schwelle bezeichnet bei höheren Anregungsdrücken demnach den Übergang zwischen kleinen inaktiven Blasen, die schwach sinusförmig schwingen, und großen, stark aufschwingenden und kollabierenden aktiven Blasen. Außerdem lässt sich erkennen, dass bei Erhöhung des statischen Druckes die Blake-Schwelle ansteigt. Starke Oszillation der Blasen wird demnach durch einen erhöhten Umgebungsdruck zu höheren Anregungsdrücken verschoben.

\subsubsection{Bjerknes-Kräfte}

Unter Anwesenheit von Druckgradienten wirken unterschiedliche Kräfte auf die sich in einer Flüssigkeit befindenden Blasen. Die einfachste und bekannteste ist die Auftriebskraft. Diese verursacht das Aufsteigen der Blasen in Flüssigkeiten, in denen durch ein Schwerefeld ein Druckgradient vorhanden ist. Wird in die Flüssigkeit Schall eingestrahlt, entstehen zeitlich veränderliche Druckgradienten. Die akustischen Kräfte, die durch diese Gradienten verursacht werden, wurden zuerst von BJERKNES [58,59] untersucht und werden deshalb Bjerknes-Kräfte genannt. Hierbei wird zwischen der primären und der sekundären Bjerknes-Kraft unterschieden.

\section{Primäre Bjerknes-Kraft}

Durch die Ausdehnung, die eine Blase im Schallfeld hat, wirken durch den Druckgradienten auf die einzelnen Punkte der Blasenoberfläche unterschiedliche Drücke 
(siehe Abbildung 2.3). Bei hinreichend kleiner Blase und nicht zu schneller Änderung des Druckfeldes bleibt die Blase durch die Oberflächenspannung dennoch nahezu sphärisch. Im Folgenden kann also weiter von einer kugelförmigen Blase ausgegangen werden. Außerdem kann bei im Vergleich zur Wellenlänge kleinen Blasen der Druckgradient $\vec{\nabla} p$ über die Blase als konstant angenommen werden. Wird mit Hilfe des Gauß'schen Satzes über alle auf die Oberflächenelemente wirkenden Kräfte summiert, ergibt sich für die auf die Blase mit dem Volumen $V$ wirkende Kraft:

$$
\begin{aligned}
\vec{F} & =-\oiint_{\partial V} p \mathrm{~d} \vec{s} \\
& =-\iiint_{V} \vec{\nabla} p \mathrm{~d} V \\
& \approx-\vec{\nabla} p \iiint_{V} \mathrm{~d} V \\
& =-V \vec{\nabla} p, \text { falls } \vec{\nabla} p \approx \text { const über das Blasenvolumen. }
\end{aligned}
$$

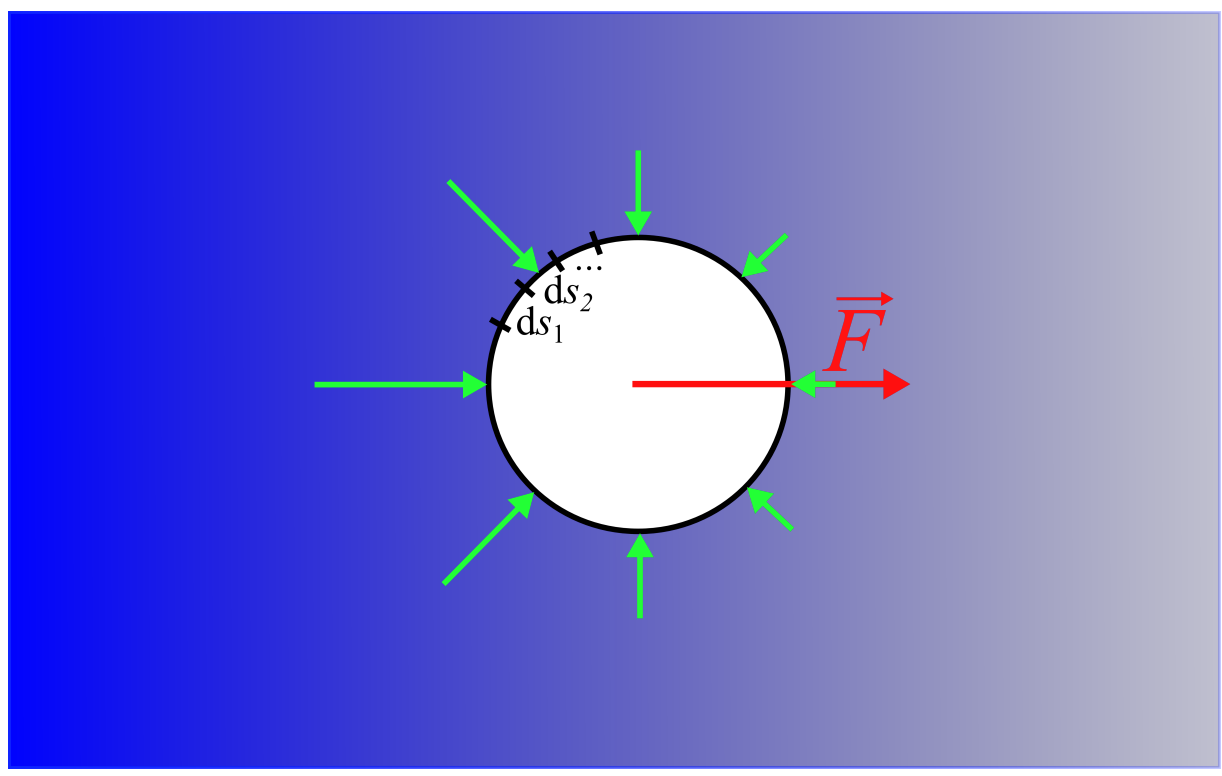

Abbildung 2.3.: Durch den Druckgradienten, der hier durch einen Farbverlauf dargestellt ist, wirken unterschiedlich starke Drücke auf die Oberflächenelemente der Blase (dargestellt durch die grünen Pfeile). Die resultierende Kraft ist durch den roten Pfeil dargestellt. 
In einem harmonisch oszillierenden Schallfeld und bei Mittelung der Kraft über eine Periode, ergibt sich für die primäre Bjerknes-Kraft

$$
\vec{F}_{\mathrm{B} 1}=-\langle V \vec{\nabla} p\rangle
$$

In einer ebenen harmonischen, stehenden Welle, in der das Druckfeld die Form

$$
p(\vec{x}, t)=p_{0}+2 p_{\mathrm{A}} \sin (k \vec{x}) \cos \left(\omega_{\mathrm{A}} t\right)
$$

habe, folgt daraus für die ortsabhängige Bjerknes-Kraft im Stehwellenfeld

$$
\begin{aligned}
& \vec{F}_{\mathrm{B} 1}(\vec{x})=-2 p_{\mathrm{A}} k V_{0} \cos (k \vec{x}) \\
& \cdot\left\langle\left(1-\frac{3 A_{0}}{R_{0}} \sin (k \vec{x}) \cos \left(\omega_{\mathrm{A}} t+\phi(\vec{x})\right)\right) \cos \left(\omega_{\mathrm{A}} t\right)\right\rangle .
\end{aligned}
$$

Das Volumen der Blase wurde dabei ebenfalls als harmonisch veränderlich gesetzt [54]:

$$
V(\vec{x}, t)=V_{0}\left[1-\frac{3 A_{0}}{R_{0}} \sin (k \vec{x}) \cos \left(\omega_{\mathrm{A}} t-\phi(\vec{x})\right)\right] .
$$

Dies ist für hinreichend kleine Anregungen der Fall (siehe auch Kapitel 2.2.1). Hierbei ist $A_{0}$ die Schwingungsamplitude, $V_{0}=\frac{4}{3} \pi R_{0}^{3}$ das Ruhevolumen der Blase mit dem Ruheradius $R_{0}, p_{\mathrm{A}} \equiv p_{1}$ der Anregungsdruck und $k=2 \pi / \lambda$ die Wellenzahl. Der Phasenwinkel $\phi(x)$ trägt der Tatsache Rechnung, dass die Schwingung der Blase nicht in Phase mit dem anregenden Schallfeld sein muss. Wenn der Ruheradius $R_{0}$ der Blase signifikant größer als der Resonanz- beziehungsweise Minnaert-Radius $R_{\mathrm{r}} \approx 2 \pi \cdot 3 \mathrm{~m} \mathrm{~s}^{-1} / \omega_{\mathrm{A}}[54,60]$ ist, der bei geringen Drücken den Übergang zwischen schwach schwingenden und stark schwingenden Blasen bezeichnet, gilt $\phi \approx \pi$. Für Blasen, deren Ruheradius signifikant kleiner ist als der Resonanzradius, gilt $\phi \approx 0$. Neben diesen Extremwerten können je nach Blasengröße beliebige Zwischenwerte auftreten. Dieser Aspekt wird allerdings hier nicht weiter betrachtet.

Für einen Ruheradius $R_{0}$ größer als der Resonanzradius $R_{\mathrm{r}}$ ergibt sich nach Anwendung der Zeitmittelung und verschiedener Additionstheoreme [54] entsprechend

$$
\vec{F}_{\mathrm{B} 1, R_{0}>R_{\mathrm{r}}}=-\frac{3 p_{\mathrm{A}} k A_{0} V_{0} \sin (2 k \vec{x})}{2 R_{0}}
$$

und für einen kleineren Ruheradius als der Resonanzradius

$$
\vec{F}_{\mathrm{B} 1, R_{0}<R_{\mathrm{r}}}=+\frac{3 p_{\mathrm{A}} k A_{0} V_{0} \sin (2 k \vec{x})}{2 R_{0}} .
$$

Dies bedeutet unterschiedliche Richtungen der Kraft je nach Größe der Blasen. Blasen, die kleiner als der Resonanzradius sind, werden also zum Druckbauch 


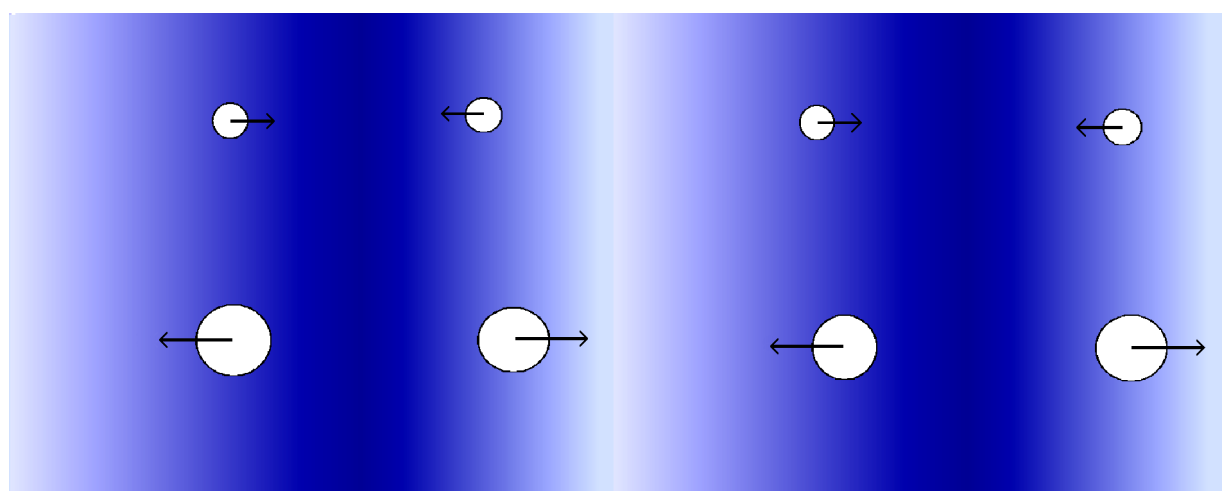

Abbildung 2.4.: Durch den Druckgradienten im Stehwellenfeld (dunkel Druckbäuche, hell Druckknoten), dessen Amplitude hier durch den Farbverlauf dargestellt ist, erfahren die Blasen, die größer als der Resonanzradius sind, eine Kraft zum Druckknoten, und diejenigen, die kleiner als der Resonanzradius sind, eine Kraft zum Druckbauch hin.

gezogen, Blasen, die größer sind als der Resonanzradius, dagegen zum Druckknoten (siehe Abbildung 2.4). Bei zeitaufgelöster Betrachtung ergibt sich durch Umkehr der Richtung des Druckgradienten zwischen Über- und Unterdruckphase auch ein Richtungswechsel der Kraft. Die Blasen schwingen also während einer Schallperiode vor und zurück. Da die in Phase schwingenden Blasen in der Unterdruckphase aber sehr viel größer sind als in der Überdruckphase und die Kraft auf die Blase somit in der Unterdruckphase größer ist, ergibt sich im Zeitmittel die oben beschriebene Richtung der Kraft und eine daraus Nettobewegung in eine Richtung. Aufgrund der Phasenverschiebung, die größere Blasen gegenüber den kleineren Blasen aufweisen, ist hier im maximal aufgeschwungenen Zustand der Druckgradient gerade umgekehrt. Daraus folgt auch die entgegengesetzte Richtung der resultierenden Kraft im Vergleich zu kleineren in Phase schwingenden Blasen. Für Blasen in beliebigen Druckfeldern kann eine allgemeinere Form für die Bjerknes-Kraft angegeben werden [61]:

$$
\vec{F}_{\mathrm{B} 1}=\underbrace{-\vec{\nabla} p(\vec{x})\langle V(t) \cos (\omega t-\phi(\vec{x}))\rangle}_{\text {Stehwelle }}+\underbrace{p(\vec{x}) \vec{\nabla} \phi(\vec{x})\langle V(t) \sin (\omega t-\phi(\vec{x}))\rangle}_{\text {laufende Welle }} .
$$

Der Term für den Anteil der laufenden Welle enthält hier einen vom Ort abhängigen Phasengradienten $\vec{\nabla} \phi(\vec{x})$, der durch die Laufzeit der Schallwelle zustande kommt.

Bei den bisherigen Ausführungen wurde die Schwingung immer als harmonisch angenommen. Bei Betrachtung der primären Bjerknes-Kraft bei nichtlinear schwingenden (und eventuell kollabierenden) Blasen, ergeben sich in Abhängig- 
keit von anregendem Druck und Ruheradius der Blase komplizierter verteilte Bereiche der Richtung der Kraft. Dies wird anhand der Grafiken in Abbildung 2.5 dargestellt (Berechnungen von METTIN [61]). Bei den später beschriebenen Ex-
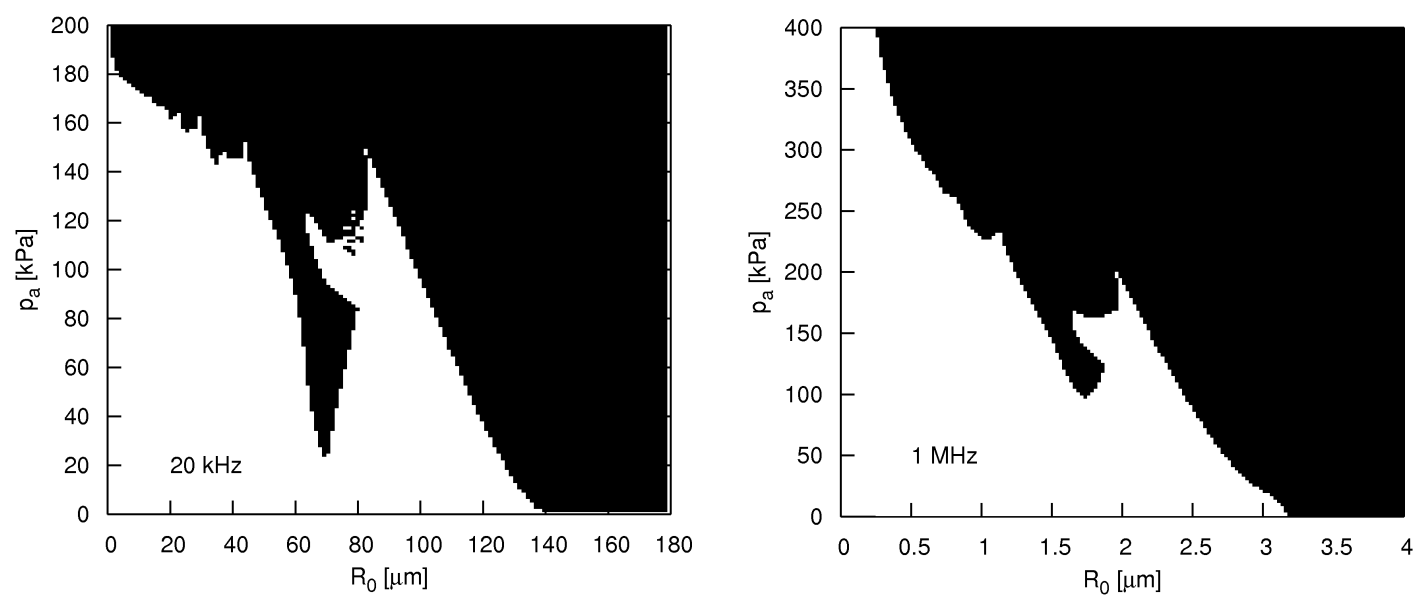

Abbildung 2.5.: Vorzeichen der mit nichtlinearen Blasenschwingungen gerechneten primären Bjerknes-Kraft für $20 \mathrm{kHz}$ und $1 \mathrm{MHz}$ in Abhängigkeit von Gleichgewichtsradius $R_{0}$ der Blase und Anregungsdruck $P_{\mathrm{A}}$. Kraft in Richtung der Druckbäuche ist weiß, Kraft in Richtung der Druckknoten schwarz gekennzeichnet. Bei $20 \mathrm{kHz}$ ist der Resonanzradius $R_{\mathrm{r}}=138 \mu \mathrm{m}$, bei $1 \mathrm{MHz}$ ist $R_{\mathrm{r}}=3.15 \mu \mathrm{m}$. Berechnungen von MetTin [61].

perimenten werden durch den hohen Druck direkt vor dem Schallwandler bei Überschreiten der Kavitationsschwelle sehr viele Blasen erzeugt, die einen großen Teil des Schalls dissipieren (siehe auch [62-65]). Dadurch entstehen ein sehr großer laufender Wellenanteil und ein starker Druckgradient, die beide starke Kräfte auf Blasen und Flüssigkeit ausüben können. In laufenden Wellen ist die BjerknesKraft für Blasen unterhalb der Blake-Schwelle sehr gering. Um die Blake-Schwelle herum nimmt sie stark zu und erreicht ebenso hohe Werte wie für nichtlinear schwingende Blasen in Stehwellen [63,66].

\section{Sekundäre Bjerknes-Kraft}

Die sekundäre Bjerknes-Kraft liegt darin begründet, dass eine schwingende Blase selber Schallwellen aussendet, die am Ort einer zweiten vorhandenen Blase einen Druckgradienten hervorrufen. Im Prinzip ist die sekundäre Bjerknes-Kraft also die gleiche Kraft wie die primäre Bjerknes-Kraft, nur dass das Schallfeld von einer zweiten Blase erzeugt wird. Schwingen beide Blasen in Phase, gibt es eine anziehende, schwingen sie gegenphasig, eine abstoßende Kraft. In einem Stehwellenfeld kann der Abstand der Blasen eine Phasenverschiebung hervorrufen, 
wenn sich eine Blase beispielsweise in einem Druckbauch befindet und die andere $\lambda / 4<d<3 \lambda / 4$ von ihr entfernt ist. Auch die endliche Schallgeschwindigkeit $c$ sorgt durch die Laufzeit des von der Blase abgestrahlten Schalls für eine Phasenverschiebung und somit für einen eventuellen Vorzeichenwechsel der Kraft.

Für die Kraft von Blase 2 auf Blase 1 gilt näherungsweise [67]:

$$
\vec{F}_{\mathrm{B} 2}^{1,2}=-\frac{\rho}{4 \pi} \frac{\vec{r}_{1,2}}{\left|\vec{r}_{1,2}\right|^{3}}\left\langle\dot{V}_{1}(t) \dot{V}_{2}(t-\tau)\right\rangle
$$

Hierbei ist $\rho$ die Dichte der Flüssigkeit, $\vec{r}_{1,2}$ der Abstand $\vec{r}_{2}-\vec{r}_{1}$ zwischen Blase 1 und Blase $2, \dot{V}_{i}$ die jeweilige Volumenoszillation und $\tau=c /\left|\vec{r}_{1,2}\right|$ die Schalllaufzeit.

Auch hier ergeben sich im nichtlinearen Fall relativ komplizierte Verteilungen der anziehenden und abstoßenden Bereiche. Derartige Abhängigkeiten sind in Abbildung 2.6 dargestellt. Für geringere Drücke ist eine schachbrettartige Struktur, verursacht durch die Phasenverschiebung der linear schwingenden Blasen, zu erkennen. Bei höheren Drücken treten die nichtlinearen Resonanzen zu Tage und rufen ein streifenartiges Muster hervor.

\subsubsection{Virtuelle Masse}

Die Bewegung einer Blase in einer Flüssigkeit erfordert die Beschleunigung des sie umgebenden Mediums. Damit kann einer Blase eine virtuelle träge Masse zugeordnet werden, hervorgerufen durch die Energieaufnahme der Strömung um die Blase bei ihrer Beschleunigung. Diese virtuelle Masse $M_{\mathrm{a}}$ entspricht im Fall einer sphärischen Blase mit dem Radius $R$ gerade der halben Masse der verdrängten Flüssigkeit [68]:

$$
M_{\mathrm{a}}=\rho V / 2=2 \pi \rho R^{3} / 3 .
$$

Die Masse des in der Blase enthaltenen Dampfes, beziehungsweise nichtkondensierbaren Gases kann meist im Vergleich zu ihrer virtuellen Masse, die unter anderem von LEIGHTON [54] und BRENNEN [55] angegeben wird, vernachlässigt werden.

Wird die virtuelle Trägheitskraft als negative Änderung des Impulses geschrieben, so folgt:

$$
\vec{F}_{\mathrm{M}}=-\frac{\mathrm{d}}{\mathrm{d} t}\left[M_{\mathrm{a}}(t) \vec{U}(t)\right]=-\frac{2}{3} \pi \rho\left[3 R^{2}(t) \dot{R}(t) \vec{U}(t)+R^{3}(t) \dot{\vec{U}}(t)\right]
$$

Sowohl eine Änderung des Blasenradius $R(t)$ als auch eine Änderung der Geschwindigkeit der Blase relativ zur Flüssigkeit $\vec{U}(t)$ rufen somit eine Veränderung 

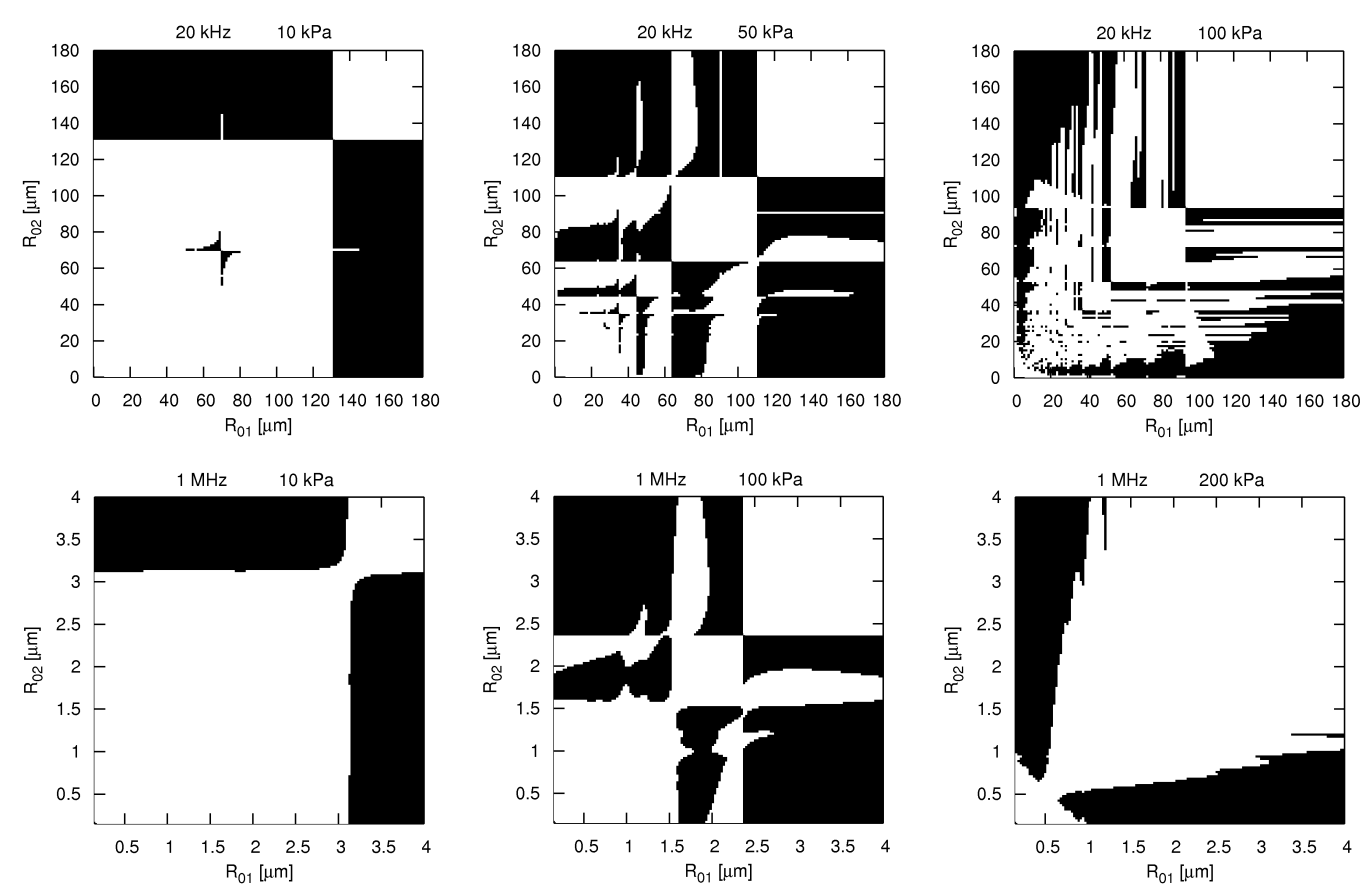

Abbildung 2.6.: Vorzeichen der mit nichtlinearen Blasenschwingungen gerechneten sekundären Bjerknes-Kraft zwischen zwei Blasen für $20 \mathrm{kHz}$ und $1 \mathrm{MHz}$ in Abhängigkeit von den Gleichgewichtsradien $R_{01}$ und $R_{02}$ der Blasen unter Vernachlässigung der Laufzeitverzögerung. Anziehende Kraft ist weiß, abstoßende Kraft ist schwarz gekennzeichnet. Bei $20 \mathrm{kHz}$ ist der Resonanzradius $R_{\mathrm{r}}=138 \mu \mathrm{m}$ und der Druck $P_{\mathrm{A}}=10,50$ und $100 \mathrm{kPa}$, bei $1 \mathrm{MHz}$ ist $R_{\mathrm{r}}=3.15 \mu \mathrm{m}$ und der Druck $P_{\mathrm{A}}=10,100$ und $200 \mathrm{kPa}$. Berechnungen von MetTin [61].

ihrer virtuellen Trägheit hervor. Bei Impulserhaltung $\left(\vec{F}_{\mathrm{M}}=0\right)$ der umströmenden Flüssigkeit führt insbesondere eine Abnahme des Volumens der Blase zu einer Zunahme der Geschwindigkeit:

$$
\dot{\vec{U}}=-\frac{3 \dot{R}}{R} \cdot \vec{U}>0 \quad \text { für } \quad \dot{R}<0
$$

Durch die sich ausbreitende Schallwelle gibt es, wie in Kapitel 2.1 bereits beschrieben, eine akustische Strömung, die in laufenden Wellenfeldern meist vom Schallwandler weg gerichtet ist. Wird eine in diesem Schallfeld schwingende und mit der Strömung mitbewegte Blase durch vorhandene Bjerknes-Kräfte, Kräfte durch Druckgradienten (zum Beispiel Auftriebskraft durch Gravitation) oder andere Kräfte in ihrer Bewegung beeinflusst, ergibt sich eine Relativgeschwindigkeit der Blase zur sie umgebenden Flüssigkeit. Dies kann bei Volumenoszillationen im 
Kollaps eine sprunghafte Bewegung der Blasen in Kavitationsfeldern wie in Abbildung 2.7 gezeigt zur Folge haben [13,69,70].

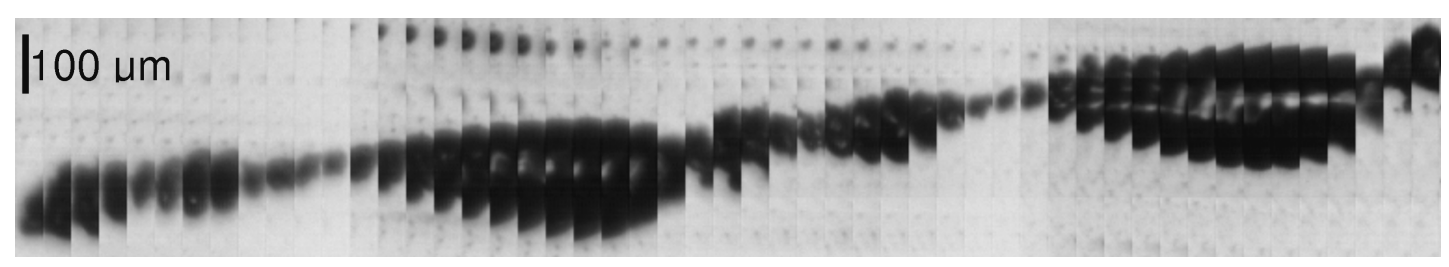

Abbildung 2.7.: Sprunghafte Bewegung einer Blase unter einer Sonotrode bei $20.61 \mathrm{kHz}$ und $185 \mathrm{~V}$ Betriebsspannung. Schmale Filmstreifen aus einem Film aufgenommen mit 25000 Bps (Bildabstand $4 \mu \mathrm{s}$ ) und $2 \mu \mathrm{s}$ Belichtungszeit aneinandergereiht. Die Zeit verläuft von links nach rechts. Sichtbar sind die Bewegung der Blase nach oben in Richtung Sonotrode und die Volumenoszillation der Blase. Bild entnommen aus [20].

\subsubsection{Reibungskraft}

Ein sich in einer Flüssigkeit bewegender Körper erfährt durch die Viskosität der Flüssigkeit eine entgegen seiner Bewegungsrichtung gerichtete Reibungskraft. Im Falle einer festen Kugel, die die umgebende Flüssigkeit mitreißt und sich langsam bewegt, so dass von einer laminaren, nicht turbulenten Strömung ausgegangen werden kann, ist die Reibung proportional zu ihrer Geschwindigkeit $\dot{\vec{X}}$ und Querschnittsfläche $\pi R^{2}$. Wenn die Flüssigkeit weit entfernt von der Blase mit der Geschwindigkeit $\vec{U}_{\mathrm{A}}$ strömt, ist die Relativgeschwindigkeit mit $\vec{U}=\vec{U}_{\mathrm{A}}-\dot{\vec{X}}$ anzugeben. Daraus folgt für die Reibungskraft:

$$
\vec{F}_{\mathrm{D}} \propto-\vec{U} \pi R^{2}
$$

Als charakteristische Größe für den viskosen Widerstand ( $\mu$ ist die dynamische Viskosität der Flüssigkeit) dient die Reynolds-Zahl. Sie ist definiert als:

$$
R e=\frac{2 \rho R|\vec{U}|}{\mu} .
$$

Kleine Reynolds-Zahlen bedeuten eine laminare, große Reynolds-Zahlen eine turbulente Strömung. Obwohl eine Gasblase im Prinzip keine feste Kugel ist, kann sie näherungsweise oft wegen der anhaftenden Verschmutzungen, die in einer realen Flüssigkeit vorhanden sind, doch als harte Kugel betrachtet werden. Die Stokes'sche Reibung für eine feste Kugel ist mit

$$
\vec{F}_{\mathrm{D}}=-6 \pi \mu R \vec{U}
$$


gegeben [71]. Wird nun ein von der Reynolds-Zahl abhängender, dimensionsloser Reibungskoeffizient $C_{\mathrm{D}}$ eingeführt [71], ergibt sich im Falle der linear mit der Geschwindigkeit wachsenden Stokes'schen Reibung:

$$
C_{\mathrm{D}} \equiv \frac{2\left|\vec{F}_{\mathrm{D}}\right|}{\rho|\vec{U}| \vec{U} \pi R^{2}}=\frac{4 \xi}{R e} .
$$

Die Reibungskraft lässt sich nun in der Form

$$
\vec{F}_{\mathrm{D}}=-C_{\mathrm{D}} \frac{\rho}{2} \pi R^{2}|\vec{U}| \vec{U}=-\xi \pi \mu R \vec{U}
$$

schreiben. Es ist zu sehen, dass der Einfluss von Re die Reibungskraft nur proportional zu $R$ wachsen lässt. Für sehr kleine Reynolds-Zahlen (Re $\ll 1)$, entspricht die Stokes'sche Reibung einer nicht oszillierenden leeren Blase dem obigen Ausdruck mit $\xi=4$ (dies wurde unter anderem von BREnNEn [55] und BATCHELOR [71] genauer beschrieben). Für eine Gasblase in einer turbulent strömenden Flüssigkeit $(R e \gg 1)$ gilt nach LEVICH [72]:

$$
\vec{F}_{\mathrm{D}}=-12 \pi \mu R \vec{U}
$$

Das heißt die Reibungskraft einer Gasblase in einer turbulent strömenden Flüssigkeit ist im Vergleich zur Reibung einer Blase in einer laminaren Strömung massiv erhöht.

\subsubsection{Oberflächeninstabilitäten}

Eine zu Beginn kugelförmige Blase ist in ihrer Schwingung nicht immer stabil. So können zusätzlich zur reinen Volumenoszillation $\dot{V}$ sogenannte Oberflächeninstabilitäten auftreten. Die einfachste Form von Oberflächeninstabilitäten sind Oberflächenmoden, die von der schwingenden Blase angeregt werden. Bei geringen Amplituden können harmonische Schwingungen höherer Ordnung auftreten, die stabil (abklingend) oder instabil (anwachsend) sein können. Der letzte Fall heißt auch parametrische Instabilität. Nimmt die Amplitude zu, können kleinere Blasen abgespalten werden oder die Blase teilt sich in viele Mikroblasen auf. Diese schließen sich in einigen Fällen wieder zu einer neuen größeren Blase zusammen. Ein Beispiel für eine an einer Sonotrode anhaftende Blase bei erst zu- und dann wieder abnehmender Schalldruckamplitude ist in den Abbildungen 2.8 gezeigt. Eine weitere Form von Oberflächeninstabilitäten tritt auf, wenn sich eine Blase in der Nähe einer Grenzfläche oder im Bereich eines sehr starken Druckgradienten, der auch durch eine Relativbewegung der Blase zur Flüssigkeit erzeugt werden kann, befindet. Grenzflächen sind zum Beispiel die Wände des Gefäßes, 


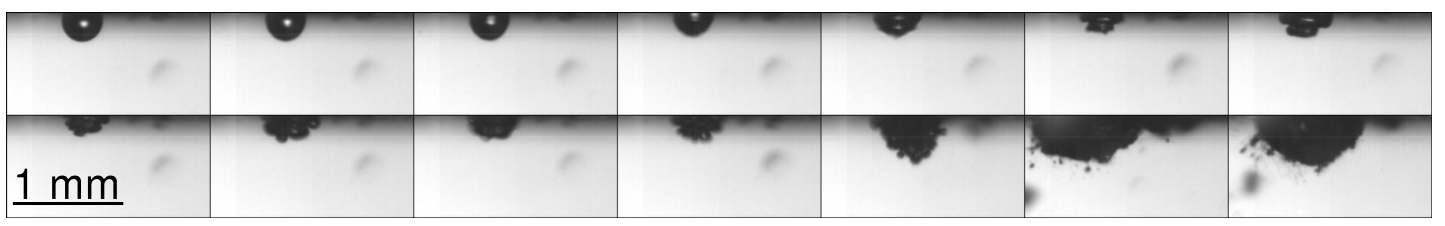

(a) Einschwingvorgang und Abnebeln kleiner Blasen.

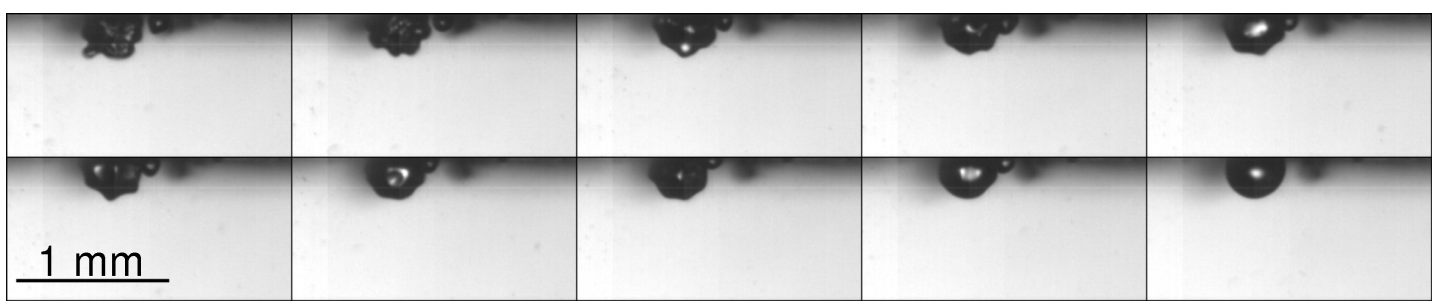

(b) Abklingen der Oberschwingungen.

\begin{abstract}
Abbildung 2.8.: Oberflächeninstabilitäten und scheinbare Volumenzunahme einer Blase direkt an der Sonotrodenspitze bei kombiniertem Schallfeld aus $500 \mathrm{kHz}$ dauerhafter Anregung und $20.61 \mathrm{kHz}$ gepulst (Pulslänge etwa $30000 \mu \mathrm{s})$. Im oberen Bild sind der Einschwingvorgang der Sonotrode und vier weitere Bilder des „eingeschwungenen“ Zustandes gezeigt, bei denen zum Teil das Abnebeln kleinerer Blasen zu erkennen ist. Das untere Bild zeigt das Abklingen der Oberflächenmoden. Film aufgenommen mit 50000 Bps und $14.3 \mu$ s Belichtungszeit. Die Zeit verläuft zeilenweise von links oben nach rechts unten. (Abstand der hier gezeigten Einzelbilder willkürlich). Bilder entnommen aus [20].
\end{abstract}

in dem sich die Kavitation abspielt, es können aber auch andere Blasen sein. So kann es passieren, dass im Kollaps die Flüssigkeit nicht von allen Seiten gleichmäßig nachströmen kann und die Blase so asphärisch kollabiert: Sie stülpt sich auf der einen Seite ein und ein Flüssigkeitsstrahl (Jet) schießt durch die Blase hindurch. Wie in Abbildung 2.9 gezeigt, bildet sich dann eine torusförmige Blase mit starken Verwirbelungen und sehr hohen Strömungsgeschwindigkeiten.

\title{
2.2.6. Simulation der Blasendynamik
}

\section{Keller-Miksis-Modell}

Das Keller-Miksis-Modell ist eine Erweiterung des Rayleigh-Plesset-Modells, dessen Ursprung auf LORD RAYleigh [74] zurückgeht und das von Plesset [75], Noltingk [76], Neppiras [77] und PORITSky [78] weiterentwickelt wurde. Es beschreibt recht genau die Radialdynamik (rein sphärische Schwingungen) von im Schallfeld schwingenden kugelförmigen Blasen. Dieses Modell reduziert die 


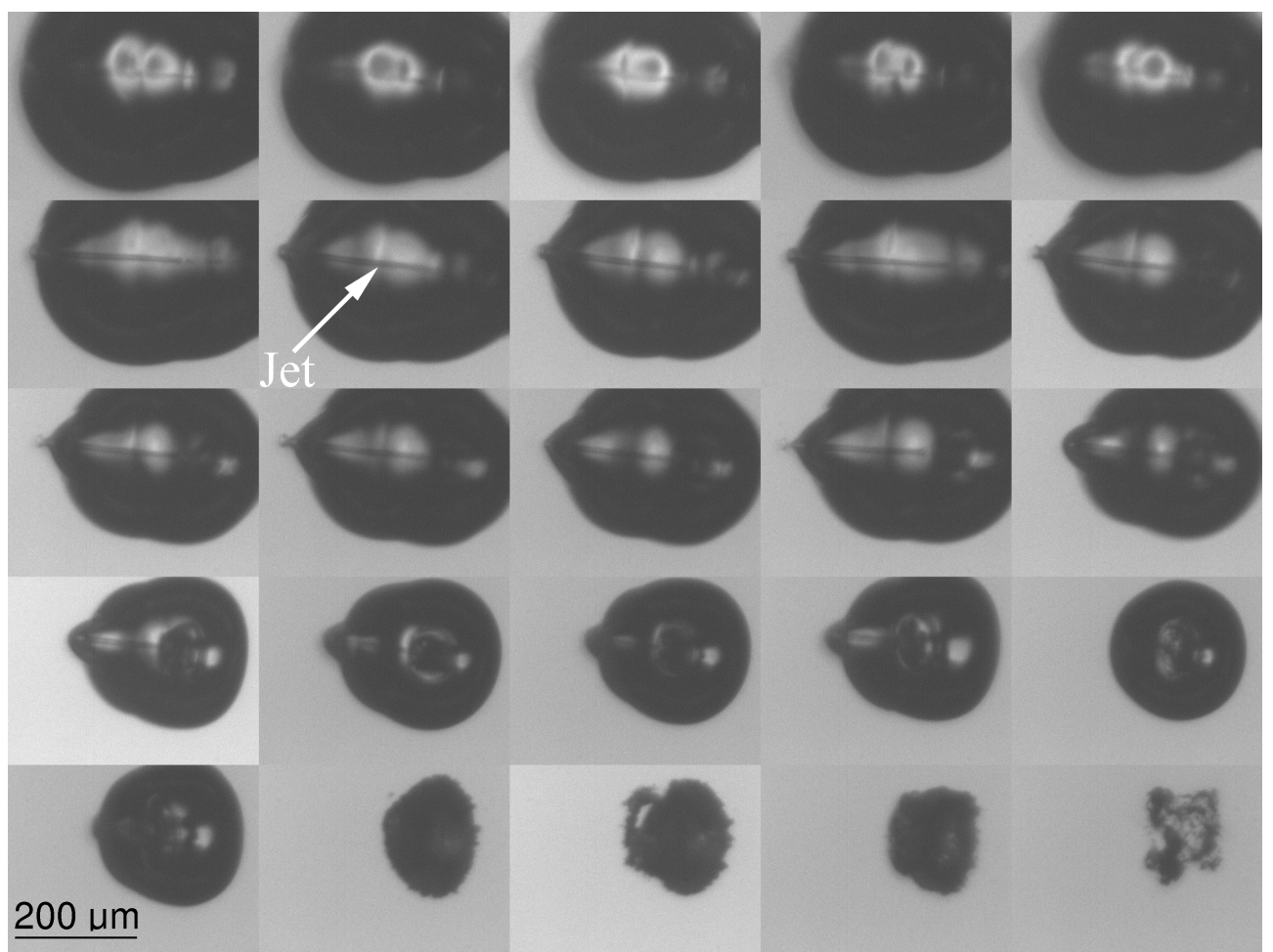

Abbildung 2.9.: Jet durch eine lasererzeugte Blase in einem Schallfeld mit starkem Druckgradienten. Es sind der Jet von rechts nach links durch die Blase, ihre toroidale Form vor und nach dem Kollaps und die Verwirbelungen nach dem Kollaps zu erkennen. Die Zeit verläuft zeilenweise von links oben nach rechts unten. Bildserie aufgenommen von Laurens Wissmann [73]. Bildabstand 1 us.

Schwingung der Blase nicht auf den harmonisch schwingenden Fall, sondern berücksichtigt auch nichtlineare Schwingungen und somit stark kollabierende Blasen. Hierbei geht es von einer schwach kompressiblen Flüssigkeit und einer rein sphärischen Oszillation aus. Unter Berücksichtigung der Schallabstrahlung und einer konstanten Anzahl von Gasmolekülen in der Blase ergibt sich folgende Gleichung [79]:

$$
\begin{aligned}
& \left(1-\frac{\dot{R}}{c}\right) R \ddot{R}+\frac{3}{2}\left(1-\frac{\dot{R}}{3 c}\right) \dot{R}^{2}=\left(1+\frac{\dot{R}}{c}\right) \frac{P_{1}}{\rho}+\frac{R}{\rho c} \frac{\mathrm{d} p_{\mathrm{g}}}{\mathrm{d} t} \\
& \text { mit } \quad P_{1}=\left(p_{0}+\frac{2 \sigma}{R_{0}}\right)\left(\frac{R_{0}}{R}\right)^{3 \kappa}-p_{0}-\frac{2 \sigma}{R}-\frac{4 \mu}{R} \dot{R}-p_{\mathrm{A}}(t) .
\end{aligned}
$$

Hierbei ist $R$ der Blasenradius, also $\dot{R}$ die Geschwindigkeit der Blasenwand, $c$ die Schallgeschwindigkeit, $\rho$ die Dichte der Flüssigkeit, $\mu$ die Viskosität, $\sigma$ die Ober- 
flächenspannung und $\kappa$ der Polytropenexponent. Der statische Druck ist mit $p_{0}$, der Gasdruck in der Blase mit $p_{\mathrm{g}}$ und der akustische Anregungsdruck mit $p_{\mathrm{A}}(t)$ bezeichnet. In Grafik 2.10 ist zu erkennen, dass sich die Kollapszeit für zuneh-

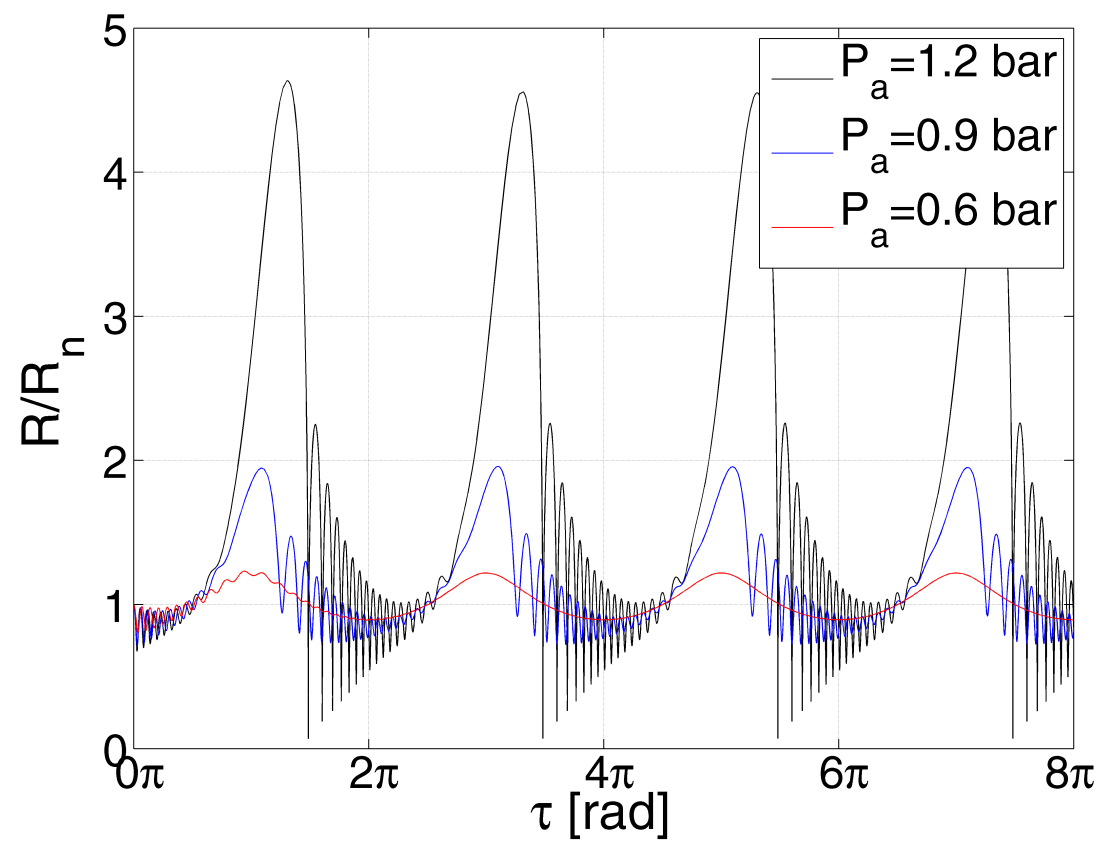

Abbildung 2.10.: Nichtlineare Blasenradialoszillation nach KellerMiksis für verschiedene Drücke bei $20 \mathrm{kHz}$ und einem Blasenruheradius $R_{0}=5 \mu \mathrm{m}$. Die Oszillationen zu Beginn sind Einschwingvorgänge der Blasen. Grafik entnommen aus [80].

mende Blasenradien, die durch Zunahme des Drucks hervorgerufen werden, nach hinten verschiebt. Ein Vergleich mit den Darlegungen in Kapitel 2.2.2 macht deutlich, dass eine Zunahme des Schalldrucks - durch die entsprechende Phasenverschiebung der Blasenschwingung gegenüber dem anregenden Schallfeld - eine Änderung der Richtung der Bjerknes-Kraft verursachen kann. Auch eine Zunahme des Blasenvolumens, durch Zusammenschluss mehrerer Blasen, kann zu einer Änderung der Richtung der Kraft führen und somit unter Umständen die Bewegungsrichtung der Blase umkehren.

\subsubsection{Blasentranslation}

Die Blasentranslation ist durch verschiedene Effekte mit der Radialdynamik der Blase gekoppelt. So hängt die Translationsbeschleunigung direkt mit der virtuellen Masse der Blase zusammen. Auch durch Strömungseffekte verursachte Oberflächeninstabilitäten haben einen Einfluss auf die Translationsgeschwindigkeit der 
Blase [81,82], da die Flüssigkeit kinetische Energie aufnimmt. Weiterhin hängt die Richtung der Bjerknes-Kraft mit der Phasenverschiebung der schwingenden Blase gegenüber dem anregenden Schallfeld zusammen, die sich ebenfalls aus der Radialdynamik ergibt.

Unter der Annahme, dass die Masse des nichtkondensierbaren Gases in der Blase vernachlässigbar klein und konstant ist, lässt sich mit dem Reibungsgesetz (2.36) (für $R e \gg 1$ ), ausgehend von den auf die Blase wirkenden Kräften und unter Berücksichtigung der Impulsbilanz der Blase, die Bewegungsgleichung für die Translation aufstellen [83]:

$$
\frac{2 \pi}{3} \rho \frac{\mathrm{d}}{\mathrm{d} t}\left(R^{3}(t) \vec{U}(t)\right)=\frac{4 \pi}{3} R^{3}(t) \vec{\nabla} P_{\infty}(\vec{X}(t), t)-12 \pi \mu R(t) \vec{U}(t) .
$$

Der Term auf der linken Seite der Gleichung (2.38) beschreibt die Änderung des Impulses (vergleiche (2.29)). Der erste Term auf der rechten Seite der Gleichung entspricht der primären Bjerknes-Kraft und der zweite trägt der Reibungskraft Rechnung. Hierbei ist $\vec{U}(t)=\vec{U}_{\mathrm{A}}(t)-\dot{X}(t)$ die Relativgeschwindigkeit der Blase als Differenz zwischen der Absolutgeschwindigkeit der Flüssigkeit und der Absolutgeschwindigkeit der Blase $\dot{\vec{X}}(t) . P_{\infty}$ ist der Druck weit entfernt von der Blase, $\vec{X}(t)$ bezeichnet die Position des Blasenmittelpunktes.

\subsection{Einfluss von Gasblasen auf akustische Strö- mungen}

Dieses Kapitel befasst sich mit dem Einfluss von Gasblasen in der Flüssigkeit auf die akustischen Strömungen. Dieser äußert sich in zusätzlicher Schalldämpfung und dem Entstehen von Mikroströmungen. Die dissipierte Schallenergie, durch die akustische Strömungen entstehen, ist eine Funktion des Schalldrucks. Dieser wiederum hängt von der Schallgeschwindigkeit $c$, der Dichte $\rho$ und der Dämpfung des Schalls, zum Beispiel durch vorhandene Blasen, ab [15,84]. Allerdings sind auch die ersten beiden Eigenschaften (Schallgeschwindigkeit und Dichte des zweiphasigen Mediums) von der Blasendichte und ihren Eigenschaften abhängig. Hierzu gehört zum Beispiel die Größe der Blase und damit die Resonanzfrequenz und die Reaktion auf den anregenden Schall. So können durch die Blasen viskose und thermische Verluste, sowie Verluste durch von den Blasen abgestrahlten Schall auftreten. Durch diese Verluste entstehen große Druckgradienten, die wiederum Auswirkungen auf die auf die Blasen wirkenden Kräfte haben (BjerknesKräfte). Zusammengefasst lässt sich sagen, dass ein hoher Anregungsdruck nach Überschreiten der Kavitationsschwelle viele Blasen erzeugt. Diese dämpfen den 
Schall stark und führen zu sehr starken Druckgradienten und wesentlich geringeren Drücken in einiger Entfernung vom Schallwandler. Außerdem werden die Blasen von der strömenden Flüssigkeit in ihrer Translationsbewegung beeinflusst, was wiederum die Blasenverteilung verändert und dadurch auch auf das Schallfeld zurückwirkt. Auch der Schallstrahlungsdruck kann die Bewegung der Blasen [85] und damit wiederum die Bewegung der Flüssigkeit beeinflussen.

\subsubsection{Schalldämpfung durch Blasen}

Allgemein lässt sich die von einer blasenhaltigen Flüssigkeit gespeicherte Energie durch die Gleichungen für Massen- und Impulserhaltung ausdrücken:

$$
\begin{aligned}
\frac{1}{\rho c^{2}} \frac{\partial p}{\partial t}+\vec{\nabla} \cdot \vec{v} & =\frac{\partial \beta}{\partial t} \\
\rho \frac{\partial \vec{v}}{\partial t} & =-\vec{\nabla} p .
\end{aligned}
$$

Hierbei ist $\beta$ das Volumen der Blasen innerhalb des zweiphasigen Mediums mit

$$
\beta(t)=N(4 / 3) \pi R(t)^{3} .
$$

$R$ ist der Radius der Blasen und $N$ ist die Anzahl der in der betrachteten Volumeneinheit vorhandenen Blasen. Eine Bilanzgleichung der durch die gesamte blasenhaltige Flüssigkeit gespeicherten mechanischen Energie lässt sich durch folgende Formel ausdrücken [15]:

$$
\begin{aligned}
\frac{1}{T} \int_{0}^{T} \frac{\partial}{\partial t}\left(\frac{1}{2} \frac{p^{2}}{\rho c^{2}}+\frac{1}{2} \rho u_{1}^{2}+N K_{1}+N 4 \pi R^{2} \sigma\right) & \mathrm{d} t+\vec{\nabla} \cdot\langle p \vec{v}\rangle \\
& =-N\left(\Pi_{\mathrm{th}}+\Pi_{\mathrm{v}}+\Pi_{\mathrm{r}}\right) .
\end{aligned}
$$

Die Terme innerhalb des Integrals beziehen sich auf die einzelnen gespeicherten Energien. So ist $p^{2} /\left(2 \rho c^{2}\right)$ die elastische potentielle, von der Flüssigkeit pro Volumeneinheit gespeicherte Energie. $\rho u_{1}^{2} / 2$ ist die kinetische Energie der Flüssigkeit, hervorgerufen durch die Schallschnelle $u_{1}$. Die kinetische Energie der Flüssigkeit pro Volumeneinheit in radialer Richtung um die Blasen $N K_{1}$ lässt sich für eine Periode $T$ auch schreiben als [15]:

$$
\frac{1}{T} \int_{0}^{T} \frac{\partial}{\partial t} N K_{\mathrm{l}} \mathrm{d} t=\left.\frac{2 \pi N}{T} \rho R^{3} \dot{R}^{2}\right|_{0} ^{T} .
$$

Die Oberflächenenergie pro Volumeneinheit wird mit dem Term $N 4 \pi R^{2} \sigma$ bezeichnet, wobei $\sigma$ die Oberflächenspannung des Wassers ist. Wird eine Zeitmittelung 
über eine Periode $T$ durchgeführt, verschwinden unter der Annahme, dass $R(t)$, $p(t)$ und $u_{1}(t)$ periodisch mit $T$ sind, alle Terme innerhalb des Integrals und es bleibt die Gleichung

$$
\vec{\nabla} \cdot\langle p \vec{v}\rangle=-N\left(\Pi_{\mathrm{th}}+\Pi_{\mathrm{v}}+\Pi_{\mathrm{r}}\right)
$$

stehen. Die akustische Intensität $p \vec{v}$ wird durch die Einkopplung des Schalls in die Flüssigkeit hervorgerufen.

Wird für die Beschreibung der Blasenschwingung das Keller-Miksis-Modell (siehe Kapitel 2.2.6) herangezogen, ergeben sich die Terme auf der rechten Seite der Gleichung [15, 62]:

$$
\begin{aligned}
\Pi_{\mathrm{th}} & =\frac{1}{T} \int_{0}^{T}-\left(p_{\mathrm{g}}\left(1+\frac{\dot{R}}{c}\right)+\frac{R}{c} \frac{\mathrm{d} p_{\mathrm{g}}}{\mathrm{d} t}\right) \frac{\partial V}{\partial t} \mathrm{~d} t, \\
\Pi_{\mathrm{v}} & =\frac{1}{T} \int_{0}^{T} 16 \pi \mu\left(R \dot{R}+\frac{R^{2} \dot{R} \ddot{R}}{c}\right) \mathrm{d} t, \\
\Pi_{\mathrm{r}} & =\frac{4 \pi}{T c} \int_{0}^{T} R^{2} \dot{R}\left(\dot{R} p+\dot{p} R+\frac{1}{2} \rho \dot{R}^{3}-\rho R \dot{R} \ddot{R}\right) \mathrm{d} t .
\end{aligned}
$$

Die Terme $\Pi_{\mathrm{th}}, \Pi_{\mathrm{v}}$ bezeichnen die durch die Blasen durch thermische oder viskose Effekte dissipierte Energie. $\Pi_{\mathrm{r}}$ ist die Energie, die durch Schallabstrahlung der Blasen während ihrer Schwingung dissipiert wird. Nun wird in den Gleichungen (2.39) das Geschwindigkeitsfeld eliminiert. Nach Ableiten ergibt sich für das Schallfeld:

$$
\nabla^{2} p=\frac{1}{c^{2}} \frac{\partial^{2} p}{\partial t^{2}}-\rho \frac{\partial^{2} \beta}{\partial t^{2}}
$$

Wird das Schallfeld als harmonisch und eben angenommen und wird Gleichung (2.43) ebenso wie die Blasenschwingung linearisiert, folgt für das komplexe Schalldruckfeld $p(\vec{r}, t)=1 / 2\left(P \mathrm{e}^{\mathrm{i} \omega t}+\bar{P} \mathrm{e}^{-\mathrm{i} \omega t}\right)$ eine Helmholtzgleichung:

$$
\nabla^{2} P+k^{2} P=0, \text { mit der Schalldruckamplitude } P .
$$

Das Quadrat der komplexen Wellenzahl, das sich aus dem Quadrat der ungedämpften Wellenzahl $k_{0}^{2}=\omega^{2} / c^{2}$ um einen komplexen Faktor ergänzt ergibt, ist nun gegeben als Dispersionsrelation:

$$
k^{2}=\frac{\omega^{2}}{c^{2}}+\frac{4 \pi R_{0} \omega^{2} N}{\omega_{0}^{2}-\omega^{2}+2 \mathrm{i} \vartheta \omega} .
$$


Hierbei ist $\omega_{0}$ die Resonanzfrequenz der Blasen und $\vartheta$ ein von den thermischen Verlusten und der viskosen Reibung abhängender Dämpfungsfaktor der linearen Blasenschwingung [62,86,87]. Auch für Schalldrücke unterhalb der Schwelle zu nichtlinearen Schwingungen der Blasen gibt es also einen komplexen und somit dämpfenden Anteil der Wellenzahl, der einen Druckgradienten und damit auch erhöhte akustische Strömungen erzeugt. Zum Berechnen der Dämpfungskoeffizienten werden für nichtlineare Blasenschwingungen oberhalb der dynamischen Blake-Schwelle nichtlineare Werte für $\Pi_{\mathrm{th}}, \Pi_{\mathrm{v}}$ und $\Pi_{\mathrm{r}}$ benutzt. Der Realteil wird weiterhin mit Hilfe der linearen Dispersionsrelation (2.44) berechnet. Der Real- und Imaginärteil lassen sich nun schreiben als:

$$
\begin{gathered}
\Re\left(k^{2}\right)=\frac{\omega^{2}}{c^{2}}+\frac{4 \pi R_{0} \omega^{2} N}{\omega_{0}^{2}-\omega^{2}} \\
\Im\left(k^{2}\right)=-2 \rho \omega N \frac{\Pi_{\mathrm{th}}+\Pi_{\mathrm{v}}+\Pi_{\mathrm{r}}}{|P|^{2}} .
\end{gathered}
$$

Mit $k=k_{\mathrm{r}}-\mathrm{i} \alpha$ lässt sich jetzt der nichtlineare Dämpfungskoeffizient bestimmen:

$$
\alpha_{\mathrm{nl}}=\frac{\rho \omega N\left(\Pi_{t h}+\Pi_{v}+\Pi_{r}\right)}{|P|^{2} k_{r}} .
$$

Unterhalb der Blake-Schwelle (siehe Kapitel 2.2.1), also bei linearer Blasenschwingung, sind die Terme $\Pi_{\mathrm{th}}, \Pi_{\mathrm{v}}$ und $\Pi_{\mathrm{r}}$ proportional zu $|P|^{2}$ und somit ist der Dämpfungskoeffizient $\alpha$ unabhängig vom Schalldruck und folgt der linearen Theorie (2.44). Um die Blake-Schwelle herum (siehe auch Kapitel 2.2.1) wächst der Dämpfungskoeffizient und damit die dissipierte Energie stark an. Oberhalb der Blake-Schwelle folgt der Dämpfungskoeffizient dem Imaginärteil, der Realteil kann hier vernachlässigt werden (siehe Abbildung 2.11).

\subsubsection{Strömungen im Kavitationsblasenfeld}

Wie im vorherigen Absatz bereits beschrieben, ist innerhalb der Blasenwolke die Dämpfung des Schalls wesentlich größer als außerhalb der Blasenwolke [15, 62]. Hierbei spielen verschiedene Effekte eine Rolle. Durch Absorption wird der Schall in blasenhaltigen Flüssigkeiten insgesamt stärker gedämpft, was zu einer erhöhten akustischen Strömung führt. Außerdem wird ein Teil der Schallenergie in Blasenoszillationen umgewandelt. Diese sorgen wiederum für eine Schallabstrahlung durch die Blasen. Des Weiteren wird ein Teil des Anregungsschalls an den Blasen gestreut. Durch diese Effekte werden unter anderem viele sekundäre Schallfelder erzeugt, die Bjerknes-Kräfte [58] zwischen den Blasen hervorrufen, und so 


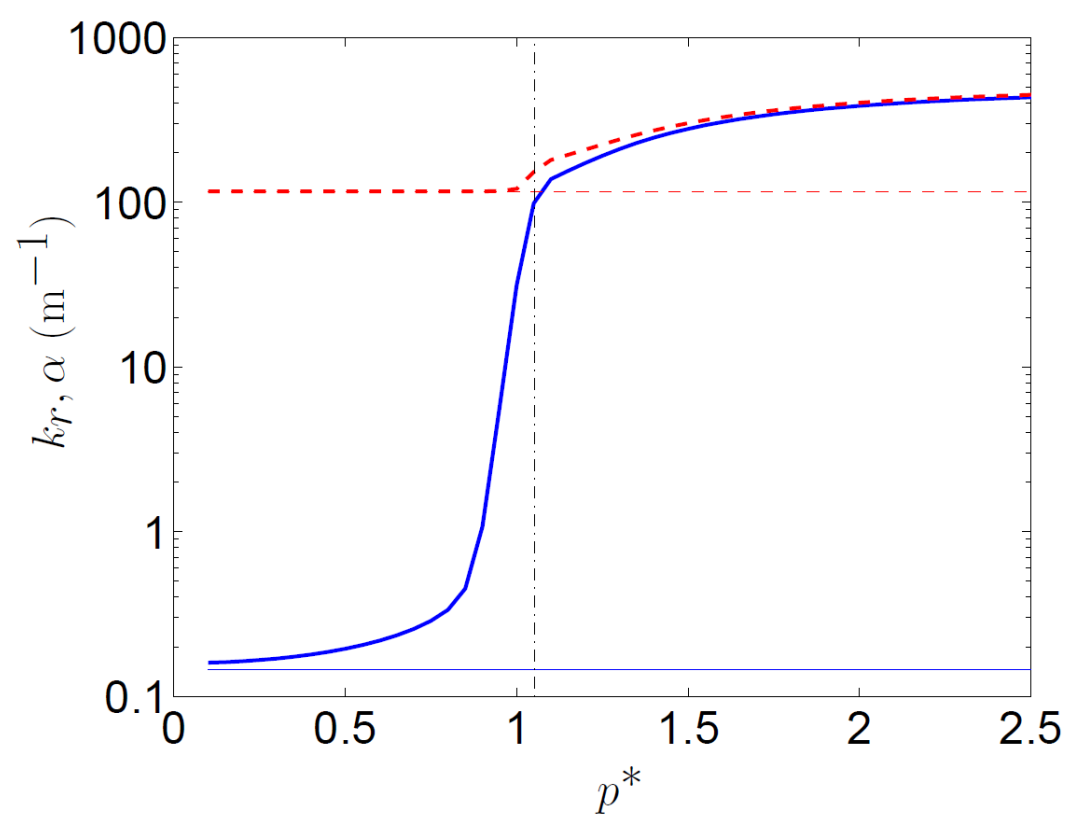

Abbildung 2.11.: Realteil (gestrichelt) und Imaginärteil (durchgezogen) der Wellenzahl $k$ aufgetragen über dem Schalldruck in bar. Gerechnet wurde für eine Blasengröße von $5 \mu \mathrm{m}$ und ein Leerraum/Volumenverhältnis von $\beta_{0}=5 \times 10^{5}$ als Funktion des Schalldrucks $|P|$. Die dünnen horizontalen Linien ergeben sich aus der linearen Theorie aus Formel (2.44). Die dicken Linien sind der Real- und Imaginärteil aus den Formeln (2.45) und (2.46). Die senkrechte Linie markiert die Blake-Schwelle. Bild entnommen aus [62].

zur Blasenbewegung und Strukturbildung beitragen [63]. Außerdem gibt es noch einen auf die Blasen wirkenden Strahlungsdruck [88], der ebenfalls für eine Bewegung der Blasen sorgt. Der Schall kann dabei an den Blasen reflektiert, durch die Blasen absorbiert oder teilweise transmittiert werden. Dieser Strahlungsdruck bewirkt eine Bewegung der Blasen von der Schallquelle weg. Aufgrund der Impulserhaltung können nun wiederum Strömungen auf die Schallquelle zu erzeugt werden. Die nichtlinearen Blasenschwingungen können zudem zu einer Verbreiterung des akustischen Spektrums durch Harmonische, eventuell Subharmonische und Rauschen führen, durch die weitere Energie aus dem ursprünglichen Schallfeld entzogen wird. Durch Blasenoszillationen [89], Oberflächenschwingungen [17], eventuelle Kollapsdynamik („Jetten“) [90] und die Translationsbewegungen der Blasen [91,92] werden zusätzliche kleinräumige Strömungen induziert, die recht hohe Geschwindigkeiten erreichen können, zum Strömungsfeld innerhalb des Blasenfeldes beitragen und letztlich durch viskose Dämpfung in Wärme dissipiert werden (siehe auch Abbildung 2.12). Alle diese Effekte sorgen für ein komple- 


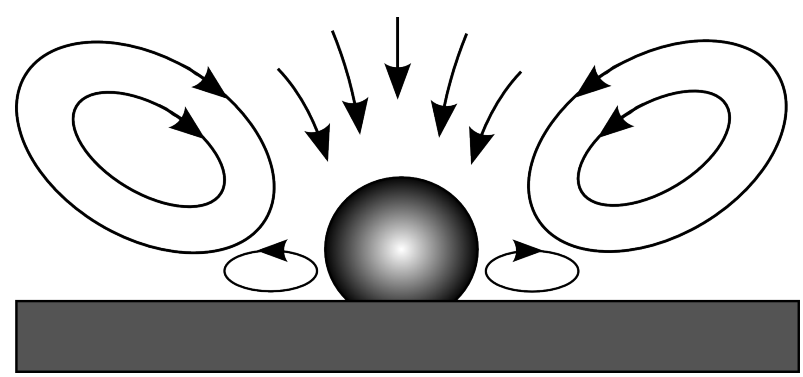

Abbildung 2.12.: Mikroströmungen um eine an einer Grenzfläche anhaftende oszillierende Blase. Zeichnung nach [4].

xes Strömungsfeld. Dieses weist nicht nur eine höhere gleichgerichtete Strömung als eine blasenfreie Flüssigkeit auf, zudem werden viele weitere Richtungen von kleinräumigen Strömungen erzeugt, die zum Teil sehr hohe Geschwindigkeiten erreichen können. Der Anteil des in kinetische Energie umgewandelten Schallfeldes steigt also bei Einsetzen von Kavitation massiv an. Als Resultat wird sich ein pseudoturbulentes Strömungsfeld hoher Energie ausbilden. 


\section{Kapitel 3 Experimenteller Aufbau}

In Abbildung 3.1 ist der gesamte experimentelle Aufbau gezeigt. Dieser Aufbau wurde in diversen Konfigurationen für die verschiedenen Experimente dieser Arbeit benutzt. So wurde für die Aufnahmen im Kilohertzbereich (kHz-Bereich) mit beiden Kameras gleichzeitig aufgenommen, um einzelne Ereignisse Effekten der Kavitation zuordnen zu können. Die Grundlagen und genauen Einstellungen sind in Abschnitt 3.1.1 aufgeführt. Auf das verwendete Ultraschallhorn (Sonotrode) und die Küvette wird in den Abschnitten 3.1.3 und 3.1.4 eingegangen. Die Konstruktion der Sonotrode wird im Anhang A näher erläutert. Abschnitt 3.2.1 beschreibt die verwendeten Gigahertz-Schallwandler (GHz-Schallwandler).

\subsection{Untersuchung von akustischen Strömungen im Kavitationsblasenfeld}

\subsubsection{Fluoreszenzmikroskop}

Zur Untersuchung der akustischen Strömungen an einer Sonotrode wurde ein spezieller Fluoreszenzmikroskopaufbau realisiert. Mit diesem ist es möglich, bei relativ großem Bildausschnitt (etwa $1.5 \times 3 \mathrm{~cm}^{2}$ ) die Kavitationsblasendynamik und die Flüssigkeitsströmungen mit 5000 Bildern pro Sekunde (Bps) simultan aufzunehmen. Diese Filme werden mit zwei Hochgeschwindigkeitskameras der Firma Photron (APX RS 150K zum Beobachten der Blasen und SA5 zum Aufnehmen der Tracer-Partikel) aufgezeichnet und auf einem Computer gespeichert. Beide Kameras sind durch einen Zweifarbstrahlteiler mit einem INFINITY K2 Fernfeldmikroskopobjektiv verbunden. Zur Beleuchtung dienen ein grüner LED-Blitz [94] und ein Coherent Verdi Laser mit 5 W Dauerstrichleistung. Für den Blitz wird eine LED des Typs Luminus Devices PT-120-G-C11_MPB mit $525 \mathrm{~nm}$ Wellenlänge verwendet. Die LED wird erst zum Verkleinern der numerischen Apertur in einen Lichtleiterstab und dann in ein Faserbündel eingekoppelt, 


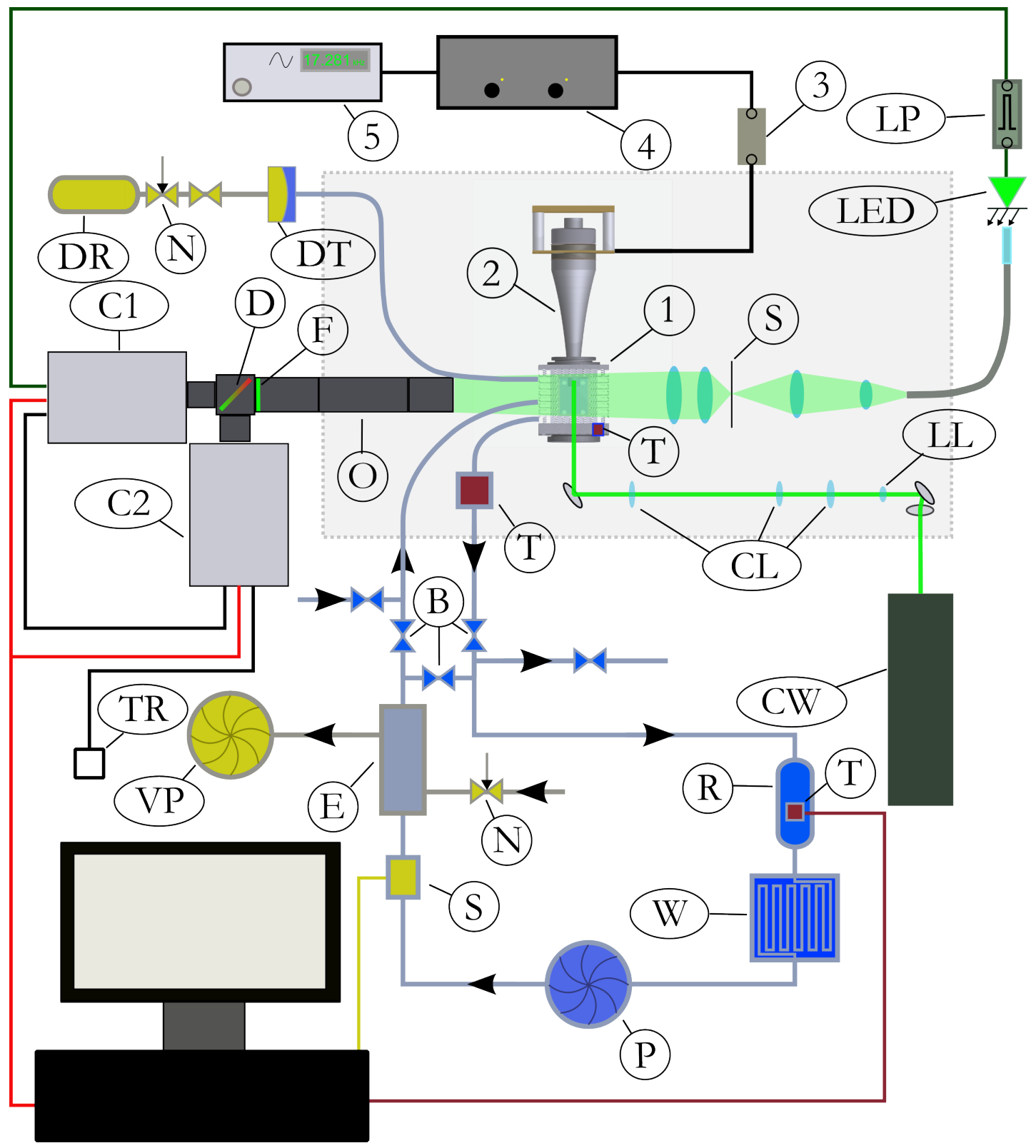

Abbildung 3.1.: Versuchsaufbau. 1: Küvette, 2: Sonotrode, 3: Impedanzanpassung, 4: Verstärker, 5: Frequenzgenerator, C1: PнотroN APX RS 150K Hochgeschwindigkeitskamera, C2: PhOtron SA5 Hochgeschwindigkeitskamera, TR: Triggerschalter, D: Zweifarbstrahlteiler, F: Laserlinien-Bandsperrfilter, O: Fernfeldmikroskopobjektiv, S: Mattscheibe, LED: Hochleistungs-LED (grün), LP: Leistungspulsgenerator, CW: $532 \mathrm{~nm} 5 \mathrm{~W}$ frequenzverdoppelter Nd-YAG Dauerstrichlaser, LL: Lichtschnittlinse, CL: Zylinderlinsen, T: Temperatursensoren, R: Wasserreservoir, W: Wärmetauscher, P: Pumpe, S: Sauerstoffsensor, N: Nadelventil, E: Membranentgasungseinheit, VP: Vakuumpumpe, B: Bypassventil, DT: Drucktauscher, DR: Druckreservoir 8 bar. 


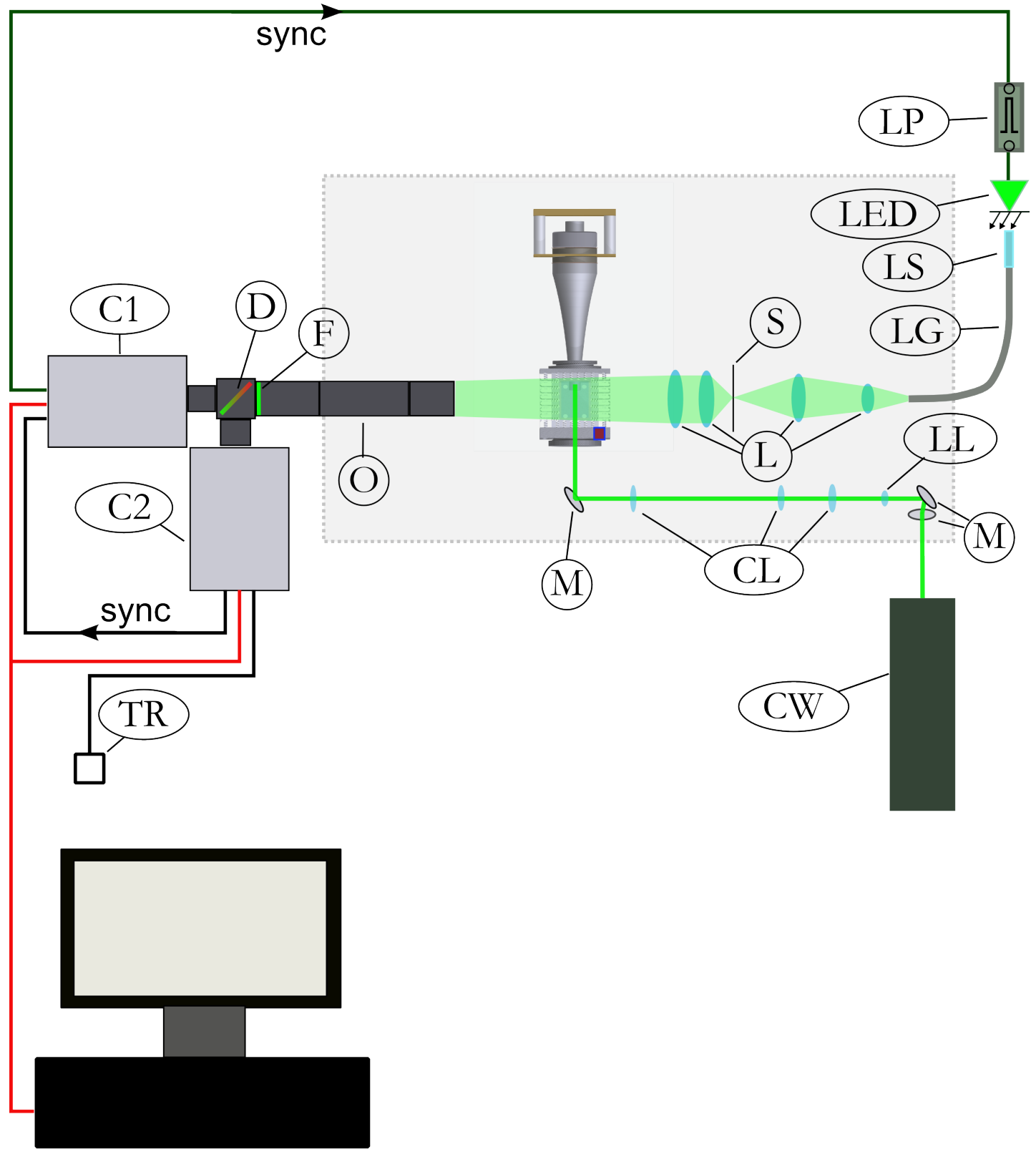

Abbildung 3.2.: Fluoreszenzmikroskopaufbau. C1: Photron SA5 Hochgeschwindigkeitskamera, C2: PнотRON APX RS 150K Hochgeschwindigkeitskamera, TR: Triggerschalter, D: Zweifarbstrahlteiler, F: Laserlinien-Bandsperrfilter, O: Fernfeldmikroskopobjektiv, L: Linsen, S: Mattscheibe, LG: Faserbündel, LS: Lichtleiterstab, LED: HochleistungsLED (grün), LP: Leistungspulsgenerator, CW: $532 \mathrm{~nm} 5 \mathrm{~W}$ frequenzverdoppelter Nd-YAG Dauerstrichlaser, M: Umlenkspiegel, LL: Lichtschnittlinse, CL: Zylinderlinsen. Der gesamte beleuchtete Teil des Aufbaus befindet sich in einer lichtdichten Kiste (grau hinterlegter Bereich). Grafik entnommen aus [93]. 
um die Aufstellung flexibel zu halten. Durch mehrere Linsen und eine Mattscheibe wird das Licht parallelisiert und die Küvette in einer Linie zur Objektivachse im Durchlicht beleuchtet. Die Dauer des LED-Blitzes lässt sich zwischen einer Mikrosekunde und einigen Millisekunden regeln. Außerdem ist die Lichtintensität einstellbar. Um die Kavitationsblasen möglichst scharf abbilden zu können, wird in diesem Versuchsaufbau eine Blitzdauer von etwa zwei Mikrosekunden verwendet. Der Laser wird über zwei Umlenkspiegel auf eine Lichtschnittlinse (LS in Abbildung 3.2) gelenkt. Das so in einer Richtung aufgeweitete Licht wird durch Zylinderlinsen noch fokussiert und durch einen Spiegel von vorne in die Küvette gelenkt. Dies ergibt einen zur Objektivachse senkrechten Lichtschnitt. Zwischen dem Fernfeldmikroskopobjektiv und dem Zweifarbstrahlteiler befindet sich noch ein Bandsperrfilter, um das Licht des Lasers vor beiden Kameras herauszufiltern und die Sensoren vor Zerstörung zu schützen. Beide Kameras werden synchronisiert und über einen externen Triggerschalter gestartet. Um das Licht der LED auf der Photron SA5 auszublenden, wird die Belichtungszeit dieser Kamera auf $1 / 6000$ s reduziert. Dies ist nötig, da die Filter alleine nicht ausreichen, um das Licht vollständig heraus zu filtern und der Kontrast zwischen Tracer-Partikeln und Hintergrund ohne diese Maßnahme zu schlecht wäre. 


\subsubsection{Wasserversorgung}

Um die Eigenschaften des Wassers im Experiment konstant zu halten, wurde ein Wasseraufbereitungskreislauf (Abbildung 3.3) installiert. Dieser macht es möglich, Temperatur und Gasgehalt in gewissen Grenzen stabil einzustellen. Dazu wird das Wasser so lange zirkuliert, bis die gewünschten Werte erreicht sind. Erst dann werden die Ventile zur Küvette geschlossen und der Bypass geöffnet (B in Abbildung 3.3). Zum Kühlen oder Erwärmen des Wassers gibt es einen Wärme-

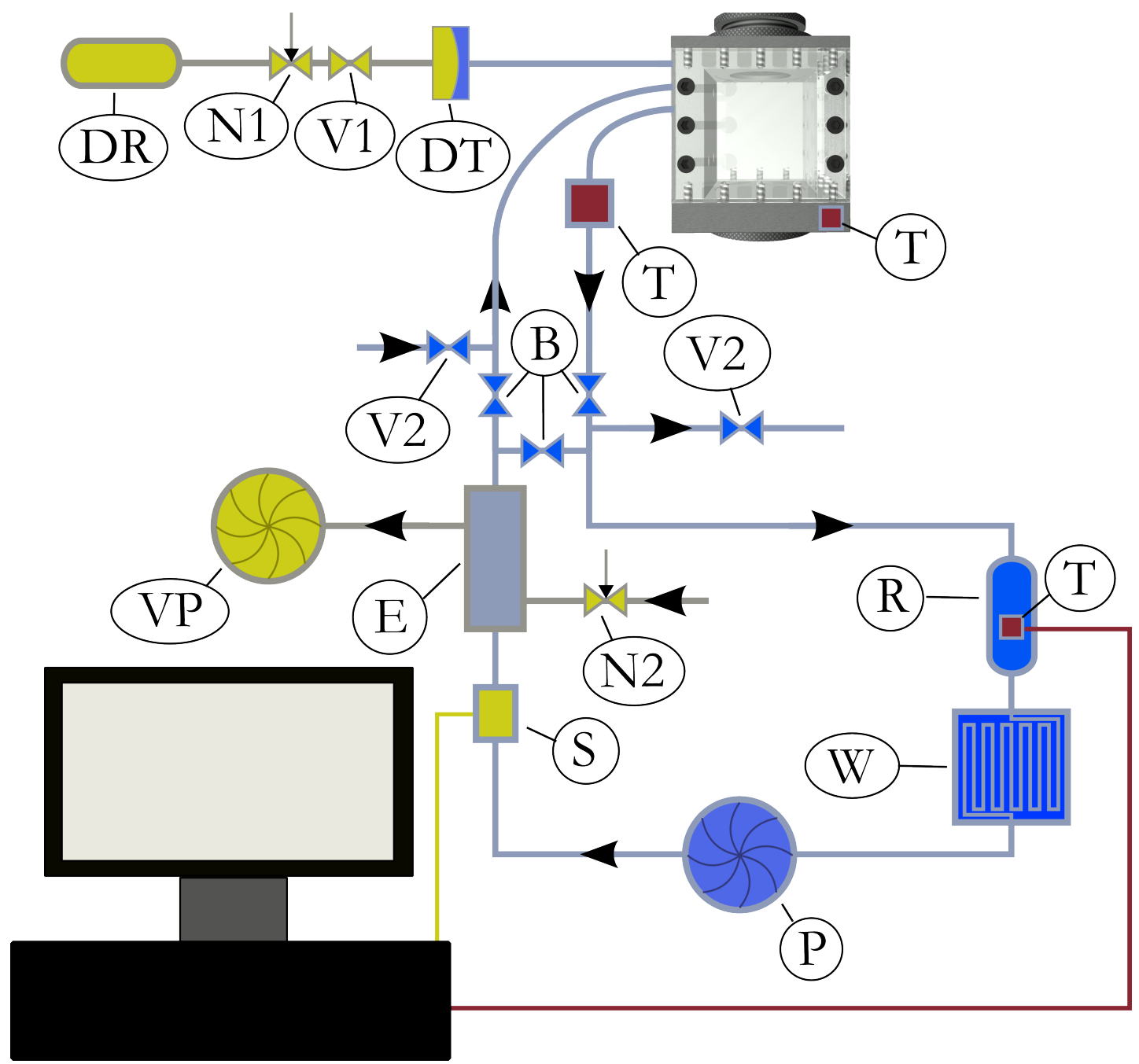

Abbildung 3.3.: Wasserkreislauf. DR: Druckreservoir 8 bar, N1: Nadelventil zum Regeln der Druckluft, V1: Elektrisches Ventil zum Schalten des Überdrucks, DT: Drucktauscher, B: Bypassventile, VP: Vakuumpumpe, E: Membranentgasungseinheit, N2: Nadelventil zum Regeln des Vakuums, S: Sauerstoffsensor, P: Pumpe, W: Wärmetauscher, R: Wasserreservoir, T: Temperatursensoren, V2: Ventile zum Befüllen und Entleeren. 
tauscher (W in Abbildung 3.3). In diesem wird das Wasser durch einen etwa $3 \mathrm{~m}$ langen Schlauch in einem Wasserbad geleitet, dessen Temperatur sich einstellen lässt. Zum Einstellen des Gasgehaltes wird eine Durchflussmembranentgasungseinheit (Membrana Liquicell 1.7×5.5 MiniModule G542, E in Abbildung 3.3) verwendet. Aus dieser wird mithilfe einer Vakuumpumpe (VACUUBRAND MZ 2c Nt, VP in Abbildung 3.3) das Gas abgepumpt. Auf der anderen Seite wird durch ein Nadelventil (N2 in Abbildung 3.3) der Gaszufluss geregelt, um beliebige Gasgehalte im Wasser einstellen zu können. Gemessen wird der Gasgehalt mit einem Sauerstoffsensor (Typ, S in Abbildung 3.3). Die Wassertemperatur wird im Wasserreservoir ( $\mathrm{R}$ in Abbildung 3.3) und kurz hinter der Küvette gemessen. Außerdem wird noch die Temperatur am Edelstahlboden der Küvette gemessen, um eine zu starke Erwärmung oder Abkühlung des Wassers durch die eingekoppelte Schallleistung oder die Kühlung der Küvette von außen während der Messung feststellen zu können. Damit es möglich ist, den Druck in der Küvette ohne eine Änderung des Gasgehaltes zu ändern, ist ein Drucktauscher (DT in Abbildung 3.3 und siehe Abbildung 3.4) an der Küvette angeschlossen. Dieser besteht aus zwei Plexiglas-Zylindern mit dazwischenliegender Gummimembran. Auf einer Seite der Membran wird ein regelbarer und elektrisch schaltbarer Überdruck angelegt. Auf der anderen Seite befindet sich das Wasser. Diese Seite ist mit der Küvette verbunden. Auf beiden Seiten ist aus Sicherheitsgründen ein Überdruckventil eingeschraubt.

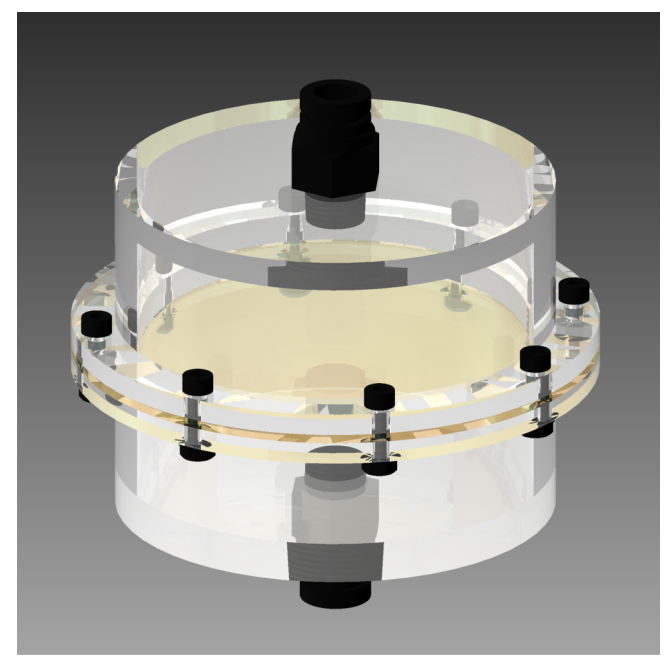

\begin{abstract}
Abbildung 3.4.: Drucktauscher zum Erzeugen des Überdrucks in der Küvette. Auf einer Seite liegt ein regel- und schaltbarer Überdruck an. Auf der anderen Seite befindet sich ein Schlauch mit Verbindung zur Küvette. Beide Seiten sind durch eine Gummimembran getrennt, um einen Gasaustausch zu vermeiden.
\end{abstract}




\subsubsection{Sonotrode}

Bei der Sonotrode (Abbildung 3.6) handelt es sich um ein Exponentialhorn (Berechnung siehe Anhang A) mit abschraubbarer Spitze, das durch zwei piezokeramische Schwinger (Piezos) angetrieben wird. Die Spitze der Sonotrode besteht aus rostfreiem Stahl, das Exponentialhorn und die Rückmasse bestehen aus Aluminium. Zwischen Exponentialhorn und Spitze lässt sich eine Gummidichtung zum druckdichten Montieren in der Küvette einklemmen. Das Signal zum Treiben der Sonotrode wird mit einem Frequenzgenerator (HEWLETT PACKARD 33120A) erzeugt und mit einem Verstärker (the t.amp TA550) verstärkt. Durch einen Koppeltransformator wird das Signal auf bis zu $600 \mathrm{~V}_{\mathrm{pp}}$ hochtransformiert (siehe Abbildung 3.5).

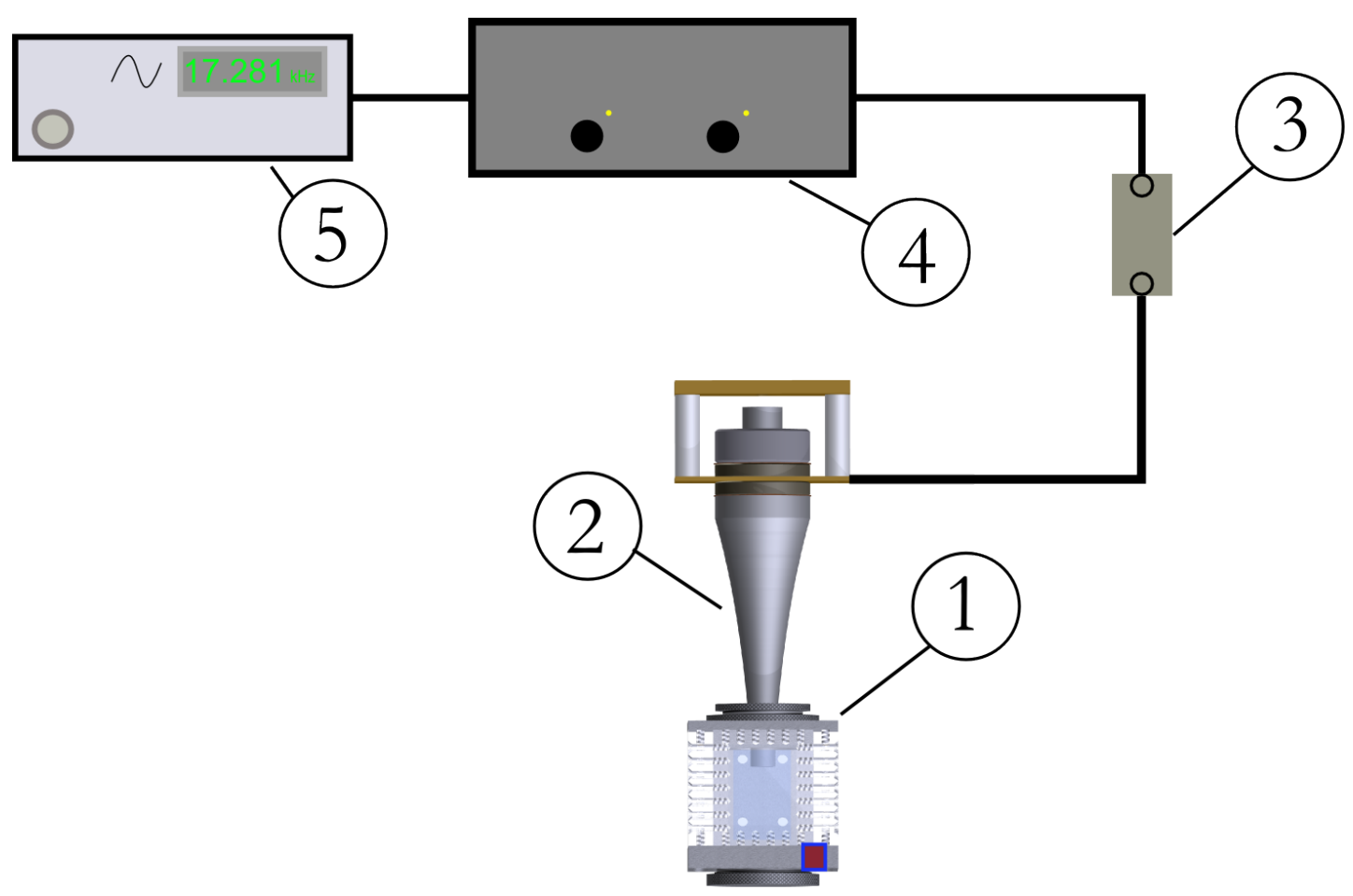

Abbildung 3.5.: Aufbau elektrisch. 1: Küvette, 2: Sonotrode, 3: Impedanzanpassung, 4: Verstärker, 5: Frequenzgenerator. 


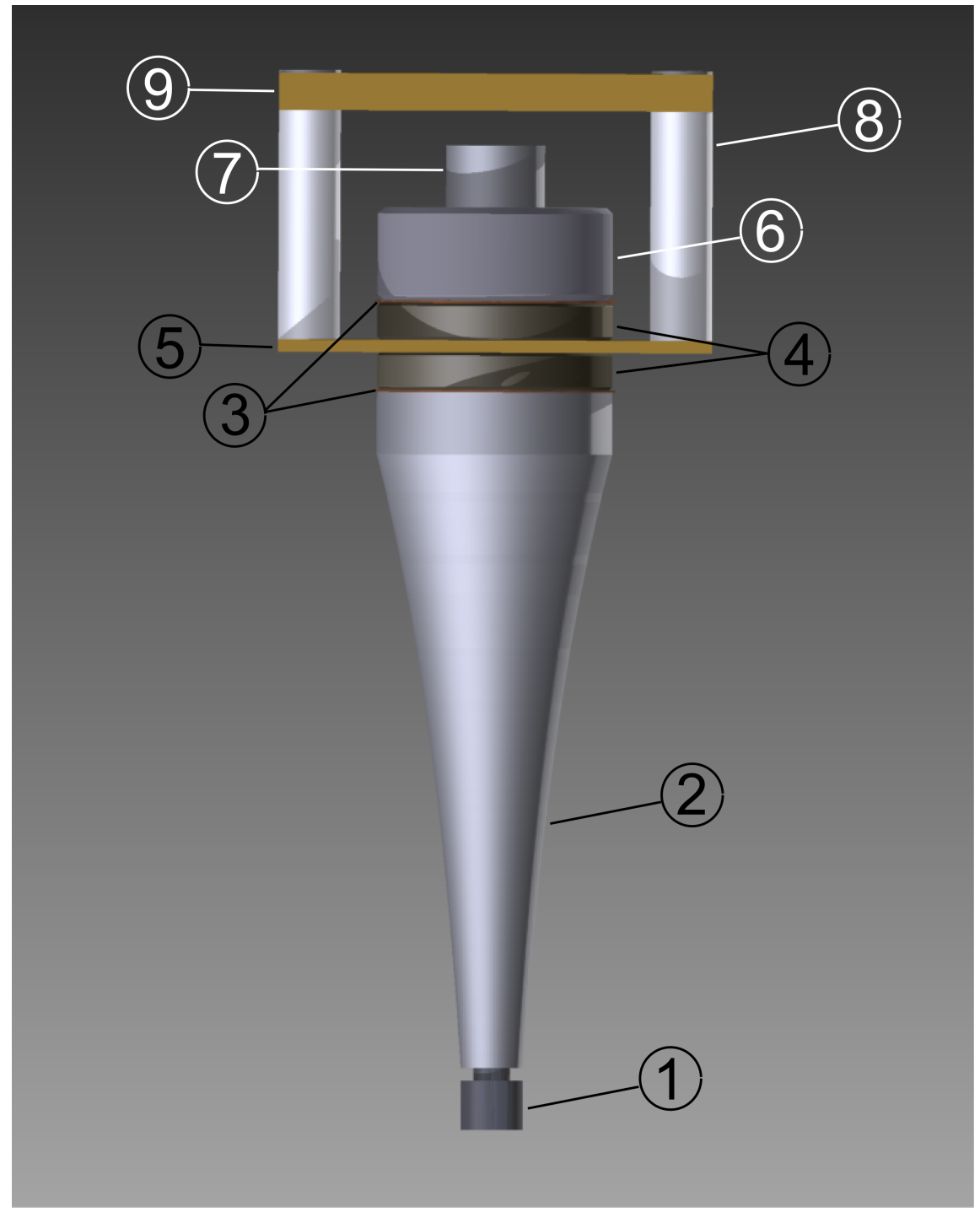

Abbildung 3.6.: Zeichnung der Sonotrode mit Halterung und abschraubbarer Spitze. In der Lücke zwischen Exponentialhorn und Stahlspitze wird die Gummidichtung eingeklemmt. Bauteile: 1: Stahlspitze, 2: Exponentialhorn (Aluminium), 3: Massekontakte (Kupfer), 4: Piezoscheiben, 5: Montageplatte und elektrischer Kontakt (Messing), 6: Rückmasse (Aluminium), 7: Schraube zum Vorspannen der Piezoscheiben, 8: Elektrisch isolierende Halterung, 9: Halteplatte (Messing).

\subsubsection{Küvette}

Die Küvette (Abbildung 3.8) besteht aus drei $1 \mathrm{~cm}$ starken Wänden aus Polymethylmethacrylat (PMMA, Acrylglas) und einer Wand aus Edelstahl. In der Edelstahlwand befinden sich die zwei Anschlüsse für die Wasserversorgung, der 


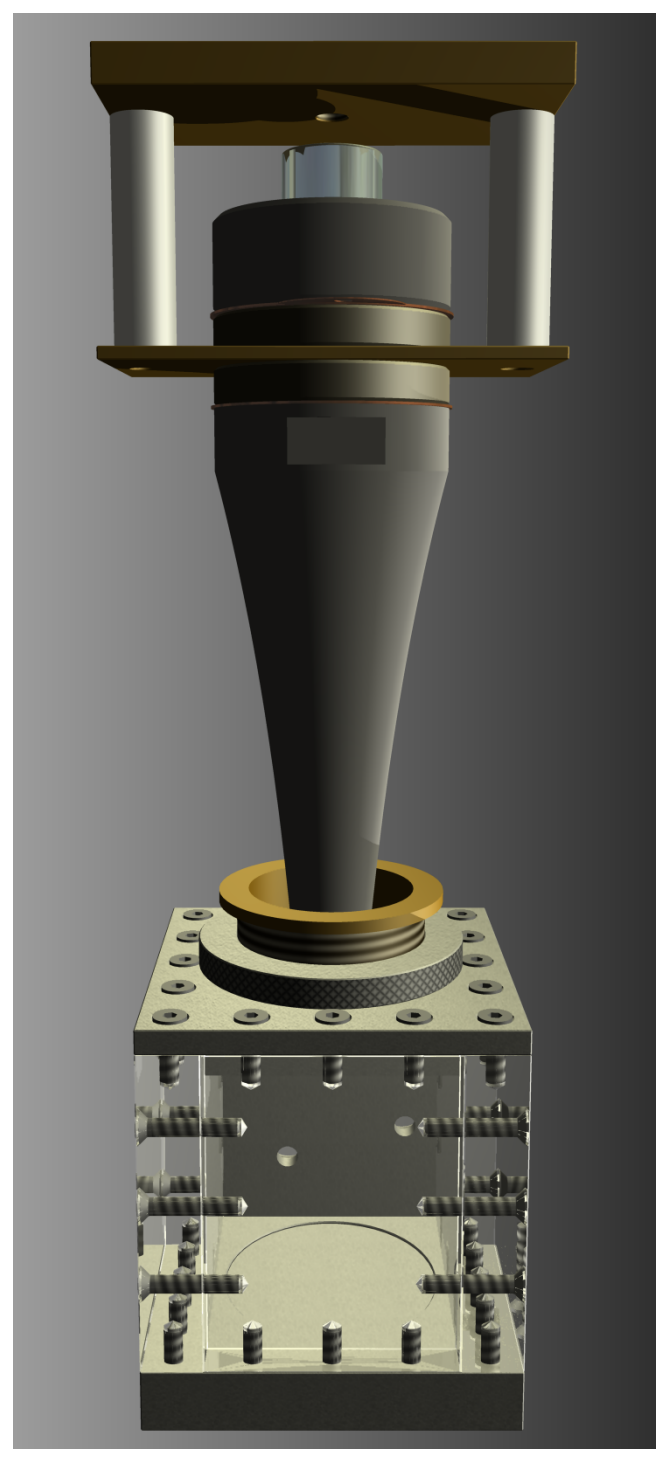

(a) Rendering

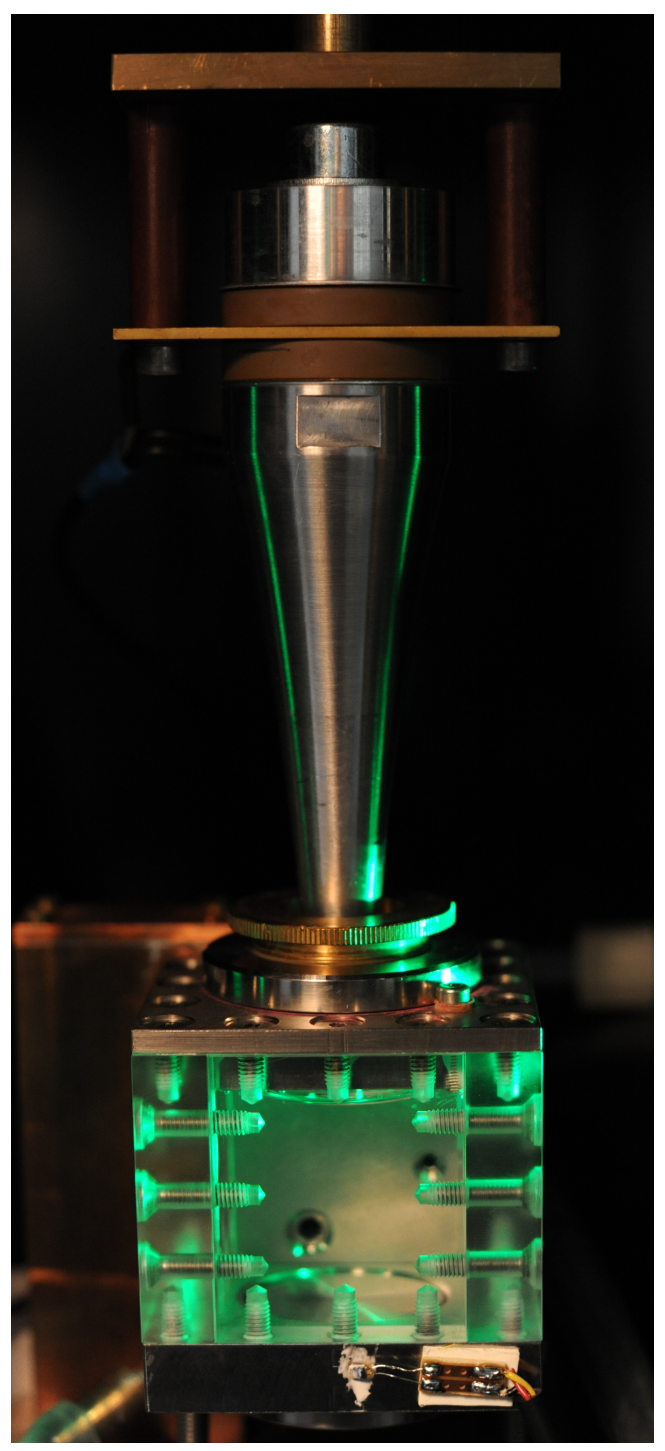

(b) Foto

Abbildung 3.7.: Rendering und Foto der an der Küvette montierten Sonotrode. Auf Foto (b) ist rechts unten einer der Temperaturfühler zu erkennen.

Anschluss für den Drucktauscher (Abbildung 3.4) und eine wärmeleitende Verbindung zum Kühlen der Küvette. Deckel und Boden der Küvette sind ebenfalls aus Edelstahl. Es lässt sich je eine Schraubkappe einschrauben. An der Oberseite befindet sich hier die Montageeinheit für die Sonotrode. Außerdem gibt es noch eine kleine mit einer Schraube verschließbare Öffnung zum Befüllen der Küvette mit Fluoreszenzpartikeln. Die gesamte Küvette ist bis zu einem Maximaldruck von etwa 3 bar Überdruck abgedichtet. 


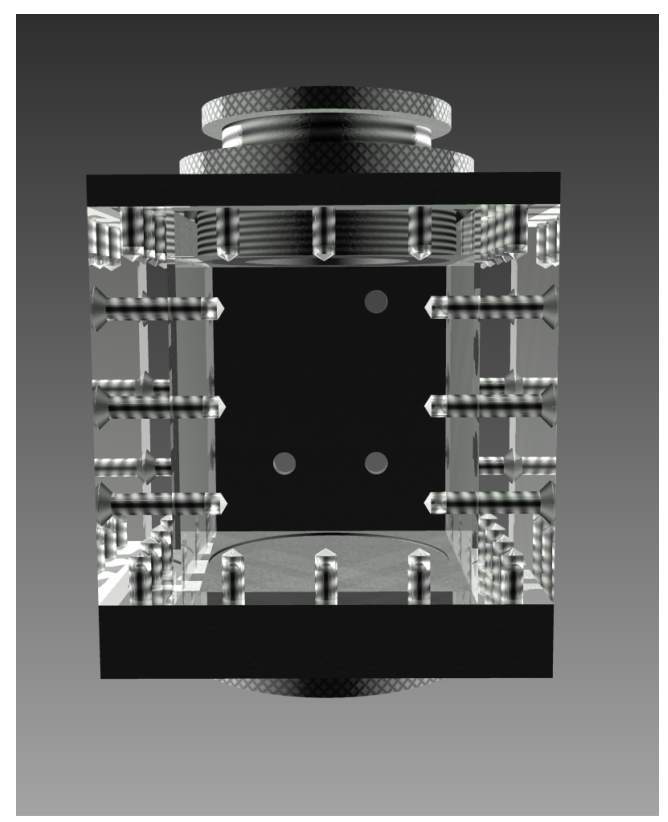

\begin{abstract}
Abbildung 3.8.: Blick von der Lichtschnittbeleuchtungsseite auf die Druckküvette. In der hinteren Wand sind die Anschlüsse für Wasser $\mathrm{Zu}$ - und Ablauf und Druck zu erkennen. Die Kamera blickt in den Versuchen von links nach rechts durch die Küvette, die Sonotrode wird an der Oberseite eingebaut.
\end{abstract}

\title{
3.2. Untersuchung von akustischen Strömungen im Gigahertzbereich
}

Zum Untersuchen der Strömungen an den GHz-Schallwandlern wurden verschiedene Methoden verwendet. Es wurden PIV-Messungen, Elektrochemiemessungen und die Visualisierung der Strömungen mit Hilfe von Tinte durchgeführt. Für die PIV-Aufnahmen und die Aufnahmen mit Tinte wurde ein Durchlichtaufbau verwendet. Bis auf den Lichtschnitt und die zweite Kamera ist der optische Aufbau identisch zu dem in Kapitel 3.1.1 beschriebenen. Nur wird hier eine rote LED des gleichen Typs verwendet (LED in Abbildung 3.9). Je nach Wahl des Bildausschnitts und der zu messenden Geschwindigkeiten, können verschiedene Aufnahmeraten der Hochgeschwindigkeitskamera (C1 in Abbildung 3.9) gewählt werden. Auch ist es möglich die Vorsatzobjektive je nach gewünschter Vergrößerung zu tauschen. Zum Betrieb der Schallwandler wird ein Frequenzgenerator (Hameg HM-8135, G in Abbildung 3.9), dessen Ausgangsleistung sich verändern lässt, und ein Verstärker (rf microwave instrumentation 15S1G4, V in Abbildung 3.9) mit bis zu 15 Watt Ausgangsleistung verwendet. Am Eingang dieses Verstärkers befindet sich ein Leistungsregelungskreis, der je nach Einstellung des Verstärker- 
potentiometers und anliegender Leistung des Frequenzgenerators automatisch die anliegende Leistung reduziert. So ist der Verstärker auf eine maximale Eingangsleistung von $0 \mathrm{dBm}$ ausgelegt. Das Verstärkerpotentiometer lässt sich im Bereich von null bis $100 \%$ regeln, wobei diese Skala recht willkürlich gewählt ist und sich nicht direkt auf die Leistung zurückführen lässt. Um die thermische Überlastung der Schallwandler zu verhindern, werden diese von der Rückseite gekühlt. Dies wird durch einen mit temperiertem Wasser durchflossenen Messingblock, auf dem ein thermisch angekoppelter Kupferstab befestigt ist, realisiert ( $\mathrm{W}$ in $\mathrm{Ab}$ bildung 3.9). Auf diesen Kupferstab werden dann die Kupferplatten geschraubt, auf denen die Schallwandlerplatinen aufgeklebt sind.

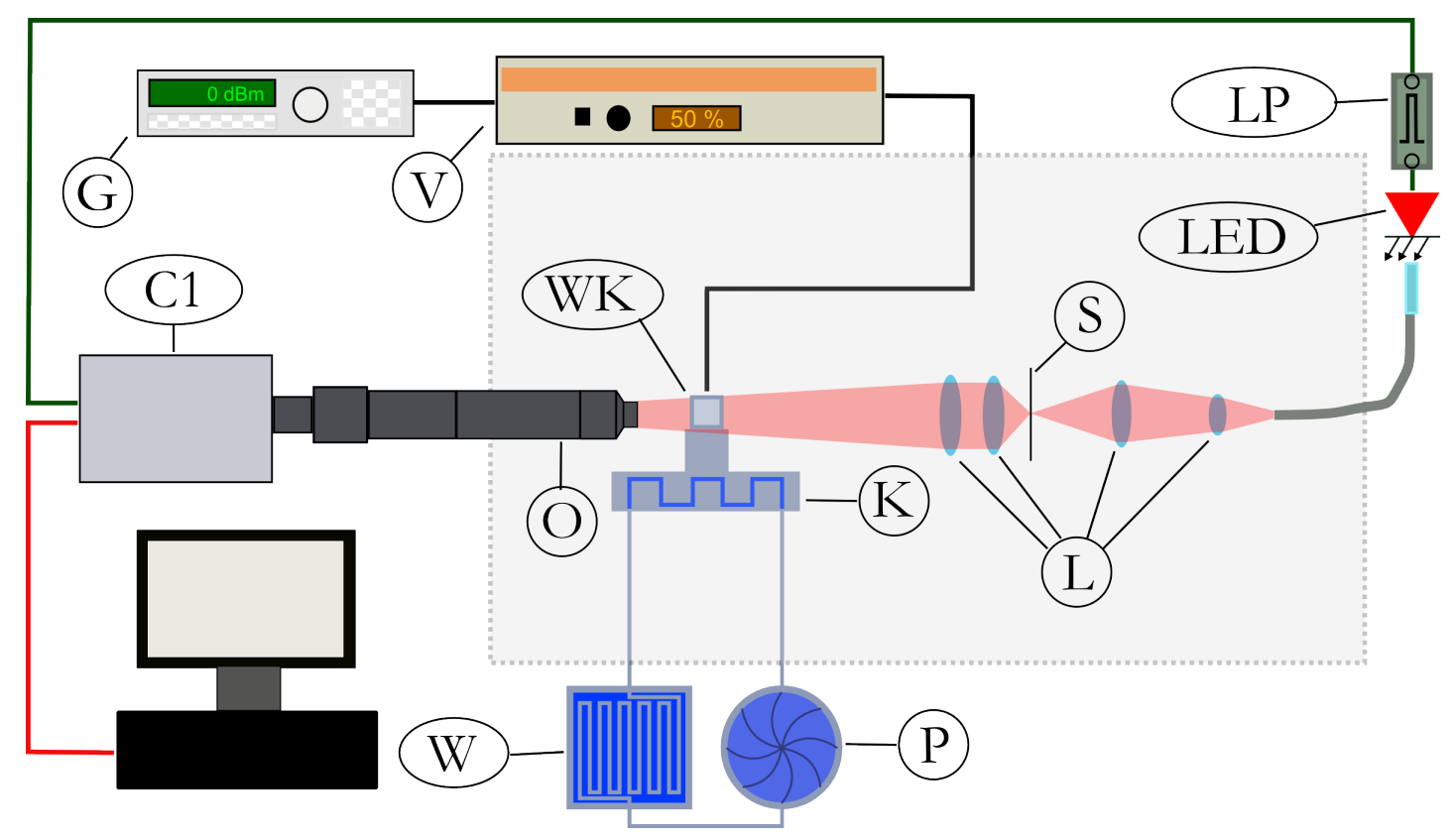

Abbildung 3.9.: Der verwendete Versuchsaufbau für die Aufnahmen im GHz-Bereich. C1: Photron SA5 Hochgeschwindigkeitskamera, O: Fernfeldmikroskopobjektiv, S: Mattscheibe, LED: Hochleistungs-LED, P: Leistungspulsgenerator, L: Linsen, P: Pumpe, W: Wärmetauscher, K: Kühlung, G: Frequenzgenerator, V: Verstärker, WK: Wandler mit Küvette.

\subsubsection{Gigahertz-Schallwandler}

Bei den verwendeten Array-Schallwandlern handelt es sich um speziell entwickelte auf einen etwa $500 \mu \mathrm{m}$ dicken und polierten Saphir-Wafer aufgebrachte Aluminiumnitridwandler. Diese Wandler, bestehend aus einem $15 \times 15$ Einzelwandler großen Array, wurden von TriQuint SEmiconductor, Inc. hergestellt. Jeder Wandler hat eine Kantenlänge von $150 \times 150 \mathrm{\mu m}^{2}$. Zwischen den einzelnen 
Wandlern ist eine Lücke von $50 \mu \mathrm{m}$ und sie sind in dieser Version elektrisch parallel geschaltet. Die stromführenden Leitungen und Elektroden der einzelnen Wandler bestehen aus Aluminium. Die gesamte Rückseite des Wandlers ist mit einer Aluminiumschicht als elektrische Masse bedampft, die großflächig mit der Platine verbunden ist, auf die die Wandler zusammen mit einer speziellen Impedanzanpassung gelötet wurden. Innerhalb der Platine befinden sich durch die Platine geführte Leiterbahnen, die wiederum mit der Platinenrückseite verbunden sind. Durch diese Maßnahmen lassen sich die Wandler durch die Platine hindurch kühlen und es können recht hohe Leistungen angelegt werden. Auf diese Platine wurde eine PMMA-Küvette (Acrylglas) mit Silikon aufgeklebt (siehe Abbildung 3.10(a)) und der Wandlerchip wurde an allen Kanten abgedichtet, um zu verhindern, dass Wasser unter diesen läuft und die Wandler beschädigt.

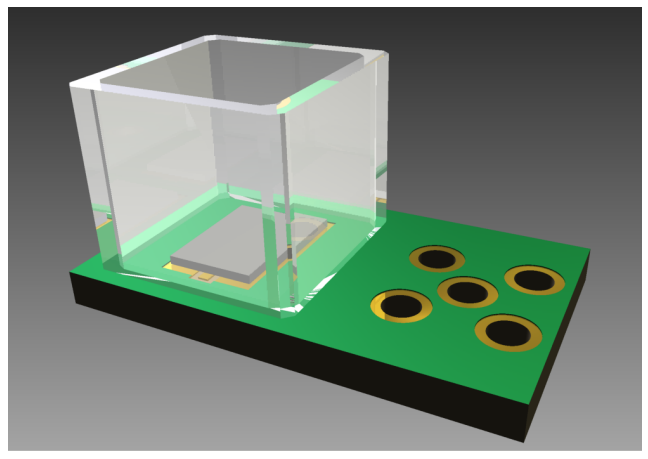

(a) Rendering

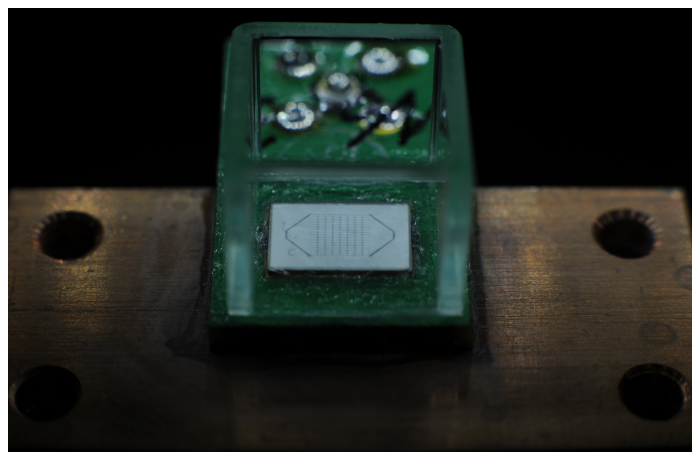

(b) Foto

Abbildung 3.10.: Rendering und Foto des Array-Wandlerchips montiert auf der Platine und einer Kupfermontageplatte mit aufgeklebter Küvette. Die Arraystruktur des Wandlers ist auf dem Foto gut zu erkennen.

Die ,zweite Generation“ der Wandler wurde von der École polytechnique fédérale de Lausanne (EPFL) entwickelt und hergestellt. Bei diesen handelt es sich um Zwillings-Schallwandler mit zwei elliptischen, in Reihe geschalteten Einzelwandlerflächen (siehe Abbildung 3.11(a)). Als Wandlermaterial wurde hier ebenfalls Aluminiumnitrid verwendet. Die stromführenden Schichten und Elektroden bestehen aus Gold und das Trägermaterial ist in diesem Fall Silizium. Da die Wärmeleitfähigkeit von Silizium wesentlich höher ist als die von Saphir, müssen bei diesen Wandlern keine besonderen Maßnahmen zur Kühlung ergriffen werden. Die Dicke des Trägermaterials beträgt hier ebenfalls $500 \mu \mathrm{m}$. Diese Wandler wurde mit Hilfe von „wire bonding“ mit einer Platine verbunden und in eine spezielle Halterung eingebaut. Dies wurde vom Fraunhofer-Institut für Biome- 
dizinische Technik (IBMT) durchgeführt (Abbildung 3.12). Auf diese Platinen wurden ebenfalls PMMA-Küvetten geklebt und mit Silikon abgedichtet (Abbildung 3.11(a)).

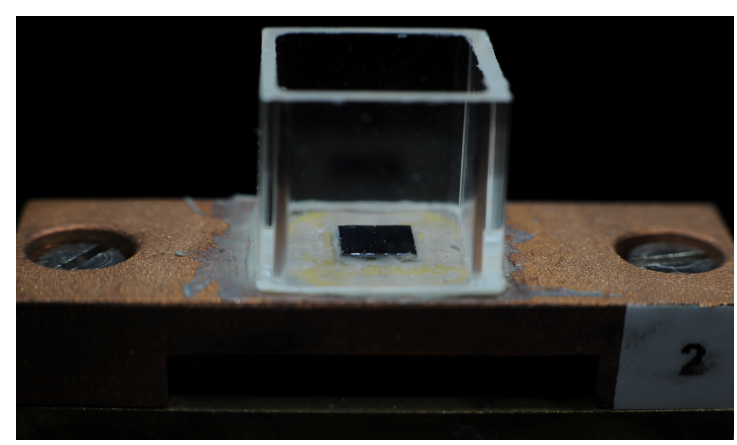

(a) Der EPFL Zwillings-Wandlerchip montiert auf der Platine und Montageeinheit mit aufgeklebter Küvette.

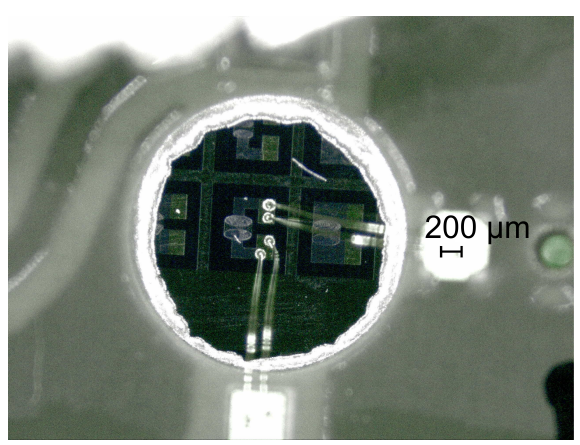

(b) Blick durch die Montageplatte auf die Unterseite des ZwillingsWandlerchips mit Golddrahtkontaktierungen.

Abbildung 3.11.: Überblickaufnahme und Blick auf die Kontaktierung des EPFL Zwillings-Wandlerchips. Mit freundlicher Genehmigung von Anette Jakob, IBMT.

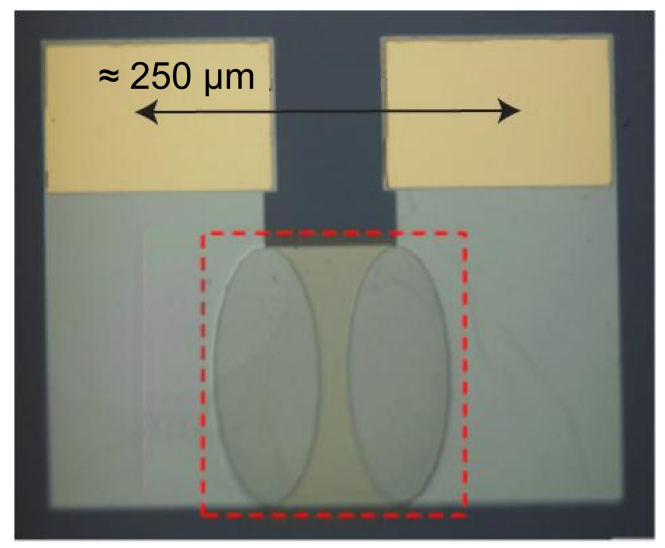

Abbildung 3.12.: Mikroskopaufnahme des EPFL Wandlerchips von der Rückseite. Die zwei einzelnen ovalen Wandler und die zwei Goldkontakte sind gut zu erkennen. Mit freundlicher Genehmigung von Anette Jakob, IBMT. 


\subsubsection{Elektrochemische Messungen}

Chronoamperometriemessungen [43] nutzen diffusionslimitierte elektrochemische Prozesse, um Strömungsgeschwindigkeiten bestimmen zu können. Hierzu wird zwischen Mess- und Referenzelektrode eine Spannung angelegt und der zeitliche Verlauf des Stroms wird gemessen. Nach einiger Zeit wird dieser Strom durch die Geschwindigkeit der Diffusion beschränkt. Durch Zuströmung frischer Flüssigkeit wird der Reaktionsprozess beschleunigt und der gemessene Strom nimmt zu. Außerdem zeigt die Diffusionsgeschwindigkeit eine Abhängigkeit von der Temperatur. Durch Anlegen von Strömungen bekannter Geschwindigkeit ließe sich eine Geschwindigkeitskalibration durchführen. Dies erfolgte im Zuge dieser Arbeit allerdings nicht.

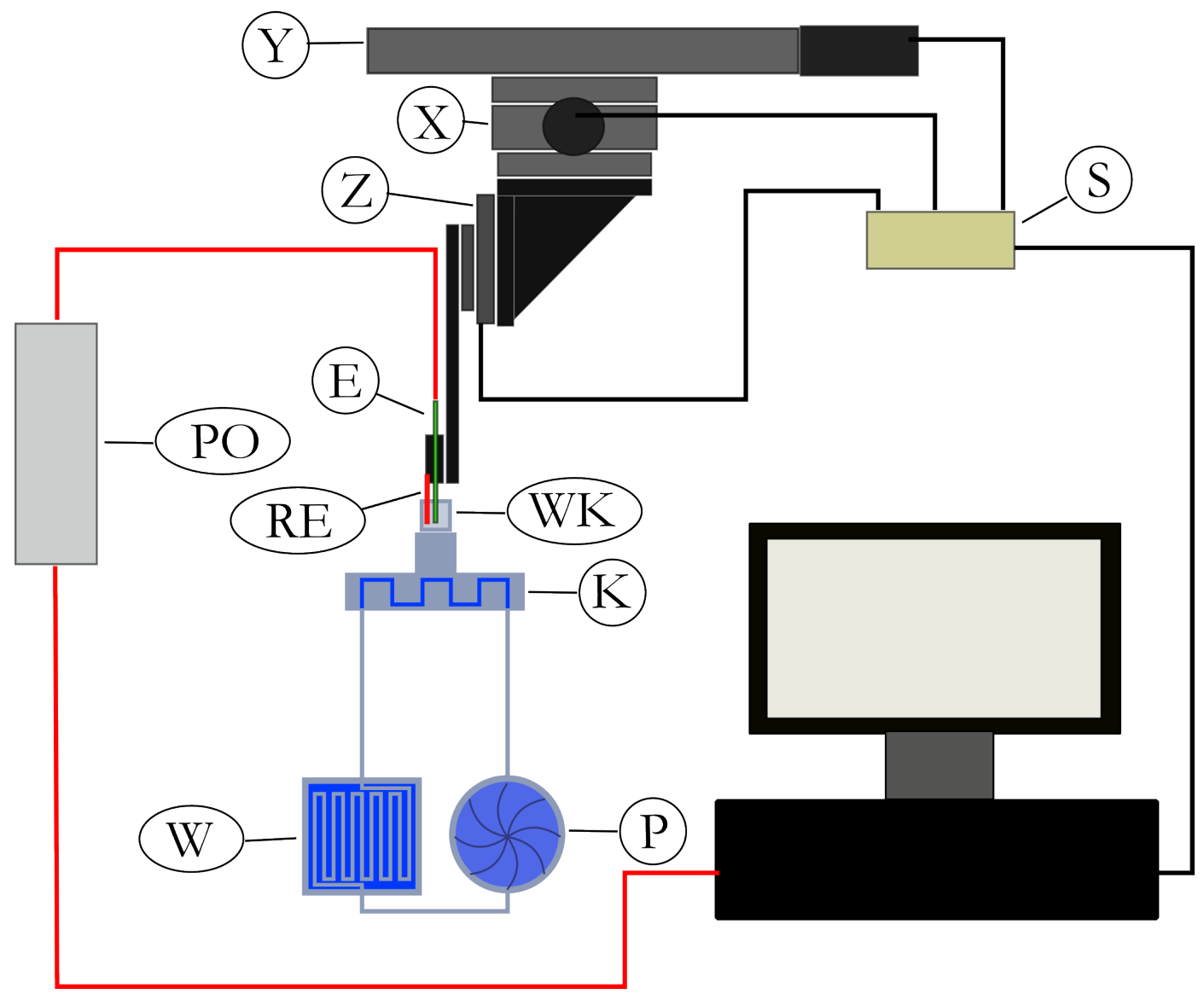

Abbildung 3.13.: Der verwendete Versuchsaufbau für die Elektrochemiemessungen. X: X-Tisch (PI M-404.2DG), Y: Y-Tisch (PI M-404.4DG), Z: Z-Tisch (PI N-661), S: Schrittmotorsteuerung (PI C-863 und E-861), E: Messelektrode, RE: Pseudoreferenzelektrode, PO: Gamry Instruments Reference 600 Potentiostat, WK: Wandler mit Küvette, K: Kühlung, W: Wärmetauscher, P: Pumpe. 
Die Chronoamperometriemessungen in dieser Arbeit werden mit einem Gamry Instruments Reference 600 Potentiostat durchgeführt (PO in Abbildung 3.13). Als Messelektroden werden Goldmikroelektroden (E in Abbildung 3.13) mit verschiedenen Durchmessern (10 und $50 \mu \mathrm{m}$ ) verwendet (siehe Abbildungen 3.14(a) und 3.14(b)). Die Pseudoreferenzelektrode (RE in Abbildung 3.13) besteht aus einem Platindraht, der in festem Abstand zur Messelektrode befestigt wird, um Abstandseffekte bei der Messung zu vermeiden.

Als Messlösung wird eine Lösung aus 0.1 M Kalium Ferrocyanid und 0.5 M Strontium Nitrat verwendet. An der Messelektrode findet dann eine Reduktion von $\mathrm{Fe}(\mathrm{CN})_{6}^{3-}$ nach $\mathrm{Fe}(\mathrm{CN})_{6}^{4-}$ statt.

Mit Hilfe einer Kombination aus Verschiebetischen von Physik Instrumente (PI) GmbH \& Co. KG lässt sich die Messelektrode exakt positionieren. Für die Verschiebung in $x$-Richtung ein M-404.2DG, in $y$-Richtung ein M-404.4DG und in z-Richtung ein Piezoantrieb mit sehr hoher Genauigkeit, N-661 (X, Y und Z in Abbildung 3.13).

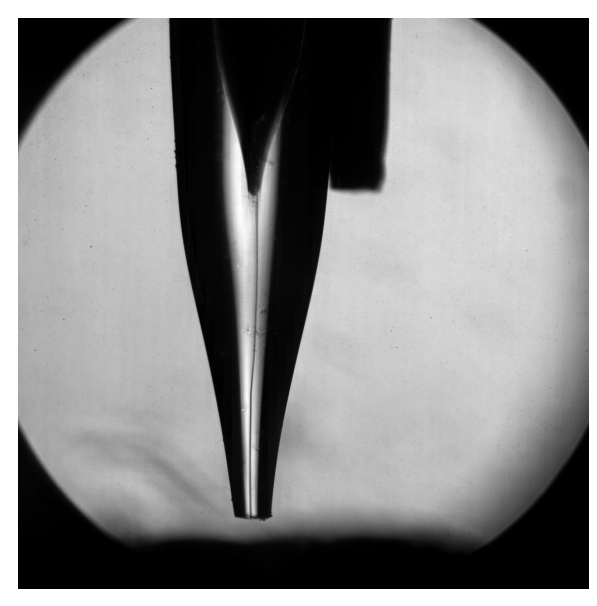

(a) $10 \mu \mathrm{m}$-Elektrode.

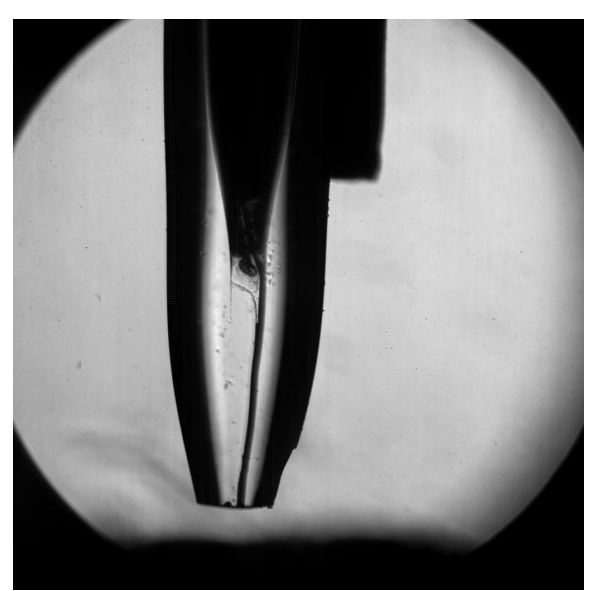

(b) 50 um-Elektrode.

Abbildung 3.14.: Aufnahmen der verwendeten Elektroden mit 10 und $50 \mu \mathrm{m}$ Durchmesser der Goldelektrode über einem Gigaschallwandler. Die Messelektrode ist innerhalb des Glaskörpers als dünner Strich zu erkennen. Rechts neben der Messelektrode befindet sich die Pseudoreferenzelektrode. 



\section{Kapitel 4 Untersuchung von Strömungen mit und ohne Kavitation}

\subsection{Kalibrationsmessungen und Methodik}

Die Strömungen an der Sonotrode (siehe Kapitel 3.1.3) wurden systematisch für verschiedene Temperaturen, Gasgehalte und elektrische Anregungsspannungen der Sonotrode untersucht. Hierbei wurde jeweils eine Messung bei Normaldruck (Umgebungsluftdruck) und eine Messung mit zwei Bar Überdruck gemacht. Der statische Überdruck wurde angelegt, um die Kavitation zu unterdrücken beziehungsweise auszuschalten. Dadurch lassen sich die Strömungen mit Kavitation mit den reinen akustischen Strömungen vergleichen und die absorbierte Energie kann abgeschätzt werden. Um bei den Messungen jeweils die gleichen Temperaturbedingungen zu haben, wurde das Wasser so lange im System zirkuliert, bis sich eine konstante Temperatur eingestellt hatte. Die Luft wurde aus dem System entfernt, indem dabei einmal vollständig entgast und der Gasgehalt danach auf den gewünschten Wert eingestellt wurde. Im Anschluss an eine Messung wurde das Wasser wieder so lange im System zirkuliert, bis sich konstante Werte eingestellt hatten. Um eine Temperatur- und Gasgehaltsänderung durch Zufluss des Wassers aus dem Drucktauscher zu vermeiden, wurde während des Zirkulierens kurzzeitig Überdruck angelegt, der das Wasser aus dem Drucktauscher dem System zurückführte. Anschließend wurde die Sonotrode auf volle Leistung geregelt bis Kavitation einsetzte, um die am Boden abgesetzten Tracer-Partikel aufzuwirbeln. Allerdings war dies bei stark entgastem Wasser und bei niedrigen Temperaturen nicht immer möglich. Erst danach wurde auf die einzustellende Spannung heruntergeregelt und kurze Zeit gewartet, um eventuelle Reste der durch die Kavitation induzierten schnellen Strömung abklingen zu lassen. Für die Aufnahmen bei zwei bar Überdruck wurden ebenso erst mit voller Leistung die Partikel vom Boden gelöst, anschließend die Spannung auf den gewünschten Wert heruntergeregelt und dann der Überdruck eingeschaltet. Ließ sich die Kavitation so nicht 
unterdrücken, wurde nach dem Aufwirbeln die Sonotrode komplett ausgeschaltet, im Anschluss der Überdruck eingeschaltet und erst dann auf die geforderte Spannung geregelt.

\subsubsection{Positionskalibration}

Um die mit den unterschiedlichen Kameras aufgenommenen Bilder möglichst exakt ausrichten zu können, wurde ein Kalibrationsgitter hergestellt, das sowohl im Durchlicht als auch im Fluoreszenzlicht zu sehen ist. Hierzu wurde ein Gitter in einen Glasobjektträger geritzt und mit Rhodamin B Fluoreszenzfarbstoff beschichtet. Anschließend wurde der Farbstoff abgewischt und blieb nur in den Vertiefungen zurück. Mit einem zweiten Objektträger, der auf den ersten aufgeklebt wurde, wurde das Gitter wasserdicht abgedeckt.

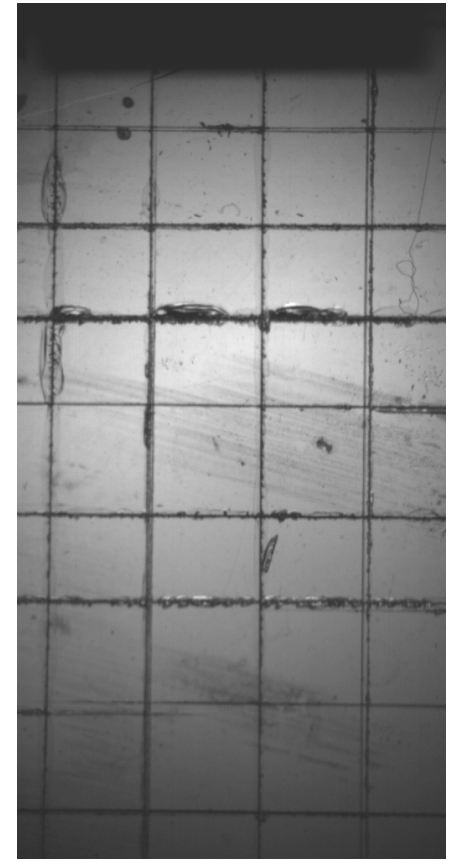

(a) Durchlichtaufnahme

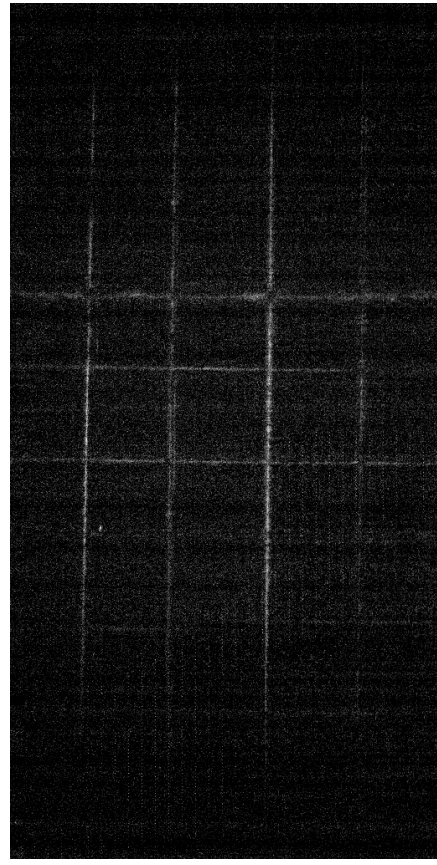

(b) Fluoreszenzaufnahme

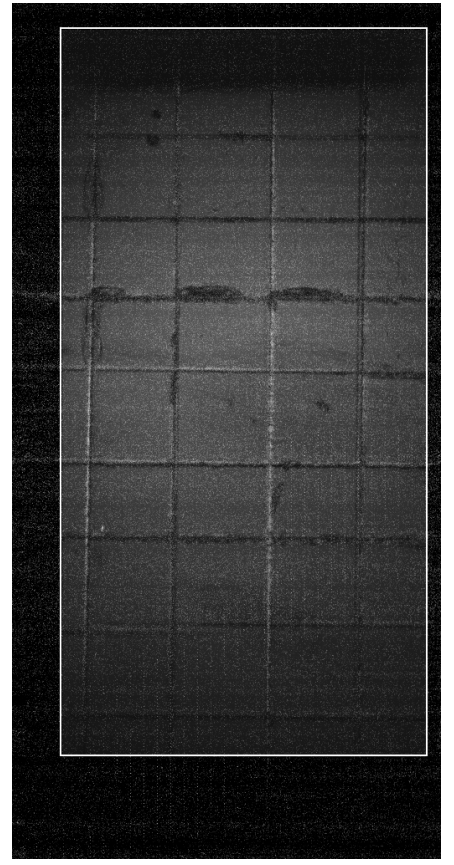

(c) Kombination beider Aufnahmen.

Abbildung 4.1.: Das benutzte Kalibrationsgitter aufgenommen mit der PhOTRON APX RS 150K Hochgeschwindigkeitskamera, der PHOTRON SA5 Hochgeschwindigkeitskamera und beide übereinandergelegt.

Die mit den Kameras aufgenommenen Bilder sind in Abbildung 4.1 gezeigt. Durch die unterschiedliche Größe der Kamerasensoren und leicht unterschiedliche Strahlengänge durch den Zweifarbstrahlteiler mussten die Kamerabilder in der Größe 
angepasst und verschoben werden. Bild 4.1(c) zeigt beide Aufnahmen in der Größe angepasst und halbtransparent übereinandergelegt. Die Linien liegen größtenteils übereinander, weichen aber teilweise durch Verzerrungseffekte leicht ab.

\subsubsection{Rohdatenverarbeitung}

In Abbildung 4.2 sind beispielhaft zwei unbearbeitete Bilder gezeigt. Um die Geschwindigkeitsvektoren der Tracer-Partikel aus Bildfolgen zu ermitteln, wurde die frei erhältliche Software PIVLab [95] Version 1.32 für Matlab [96] benutzt. In dieser Software lassen sich Teilbereiche, in denen keine oder zu wenige TracerPartikel vorhanden sind (zum Beispiel dort, wo sich die Sonotrodenspitze befindet) maskieren. Es wurde eine lineare FFT-Fenster Deformation benutzt. Bei den Filmen ohne Kavitation wurde ein Durchgang mit einer Fenstergröße [97, 98] von 64 Pixeln und einer Verschiebung von 16 Pixeln durchgeführt. Bei sehr langsamen Strömungen wurden Zwischenbilder des Filmes weggelassen, um eine klare Bewegung der Tracer-Partikel zu erhalten und das Rauschen zu minimieren. Bei den Filmen mit Kavitation wurden zusätzlich ein zweiter Schritt mit 32 Pixeln Fenstergröße und 16 Pixeln Verschiebung und ein dritter Schritt mit 16 Pixeln Fenstergröße und 8 Pixeln Verschiebung durchgeführt, um die Strömungen direkt vor der Sonotrode besser aufösen zu können. Eine vorherige Bildbearbeitung fand weder bei den Aufnahmen mit noch bei den Aufnahmen ohne Kavitation statt. In der Software wurden die Geschwindigkeiten mit Hilfe der sichtbaren Sonotrodenstirnfläche und der Aufnahmerate kalibriert. Alle weiteren Bearbeitungen der Daten wurden in MATLAB durchgeführt.

Die mit der Photron APX RS 150K erhaltenen Bilder wurden verschiedenen Bildbearbeitungsprozessen unterzogen, um die Kavitationsblasenwolken besser sichtbar zu machen. So wurde der Hintergrund (ein Bild aufgenommen unter den ansonsten selben Bedingungen bei 2 bar Überdruck) abgezogen und entweder der Kontrast erhöht oder ein Schwellwert angelegt. Mit MatLAB wurden die Filme eingelesen. Um die Blasenwolken und das dazugehörige Strömungsfeld darzustellen, wurden die mit Hilfe von PIVLab erhaltenen Geschwindigkeitsvektoren in die Filme eingezeichnet. In Matlab liegen die Bilder eines Filmes als Array aus einzelnen Matrizen vor. In diesen Matrizen sind die Grauwerte als Zahlen zwischen 0 und 255 dargestellt. Durch Anlegen eines Schwellwertes lässt sich die Anzahl der ,schwarzen“ Pixel ausgeben. Durch dieses Verfahren lässt sich das Volumen der vorhandenen Blasen in den Aufnahmen der Kavitationsblasenfelder grob bestimmen. Obwohl hintereinanderliegende Blasen ausgeblendet werden, ist diese Methode geeignet, um die durchschnittliche Schwingungsfrequenz der Blasen oder deren Phase darzustellen. 


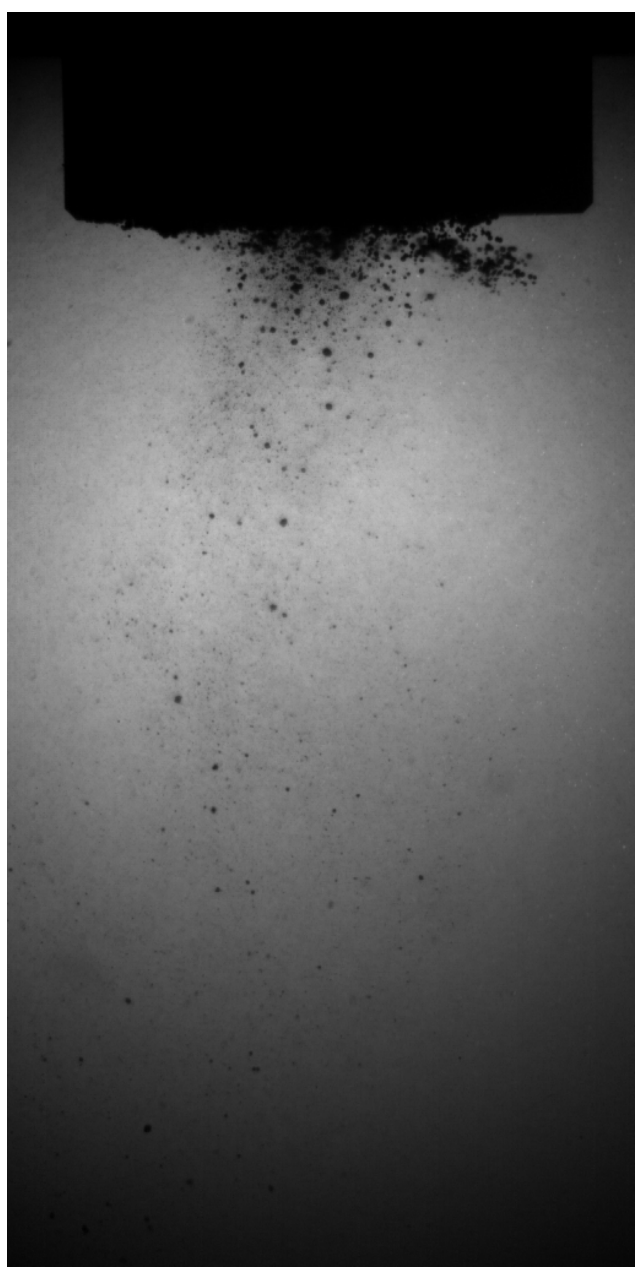

(a) Durchlichtaufnahme

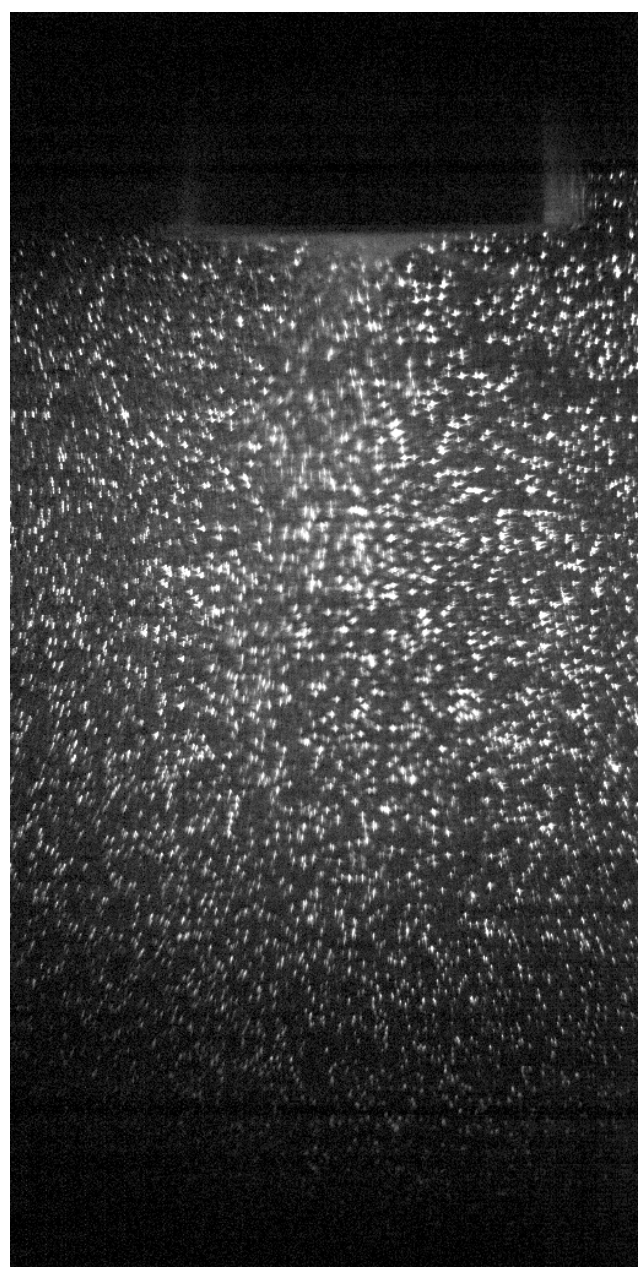

(b) Fluoreszenzaufnahme

Abbildung 4.2.: Beispiel eines unbearbeiteten Bildes aus einem Film. 4.2(a) Bild aufgenommen mit der Photron APX RS 150K Hochgeschwindigkeitskamera. Aufgenommen mit 5000 Bildern pro Sekunde, 1/5000 s Belichtungszeit und etwa $2 \mu \mathrm{s} \mathrm{LED-Blitz.} \mathrm{4.2(b)} \mathrm{Bild} \mathrm{aufgenommen} \mathrm{mit}$ der Photron SA5 Hochgeschwindigkeitskamera. Aufgenommen mit 5000 Bildern pro Sekunde, 1/6000 s Belichtungszeit und etwa 4 W Laserleistung.

\subsubsection{Kalibrationsmessungen der Auslenkung}

Um den akustischen Wechseldruck an der Sonotrode theoretisch abschätzen zu können, wurde die Auslenkung der Sonotrode über der Spannung gemessen. Dazu wurde eine höhere optische Vergrößerung als für die sonstigen Aufnahmen verwendet. Außerdem wurde mit einer Aufnahmegeschwindigkeit von 100000 Bildern pro Sekunde gearbeitet. In den aufgenommenen Filmen wurde mit MATLAB in 
jedem Bild die Anzahl der schwarzen Pixel bestimmt, der Minimalwert jedes Filmes davon abgezogen und in eine Auslenkungsamplitude umgerechnet. Die erhaltenen Ergebnisse bei den verschiedenen Temperaturen, mit und ohne Überdruck, sind in den Abbildungen 4.3 bis 4.5 bei $17.281 \mathrm{kHz}$ Anregungsfrequenz dargestellt. Für $17.381 \mathrm{kHz}$ sind die Ergebnisse in den Abbildungen 4.6 und 4.7 gezeigt. Hier wurde für $30^{\circ} \mathrm{C}$ allerdings keine Kalibrierung durchgeführt. Alle Auslenkungskalibrierungen wurden bei voll entgastem Wasser durchgeführt, um die Kavitation $\mathrm{zu}$ unterdrücken und somit zu vermeiden, dass sich die Aufnahme wegen einer Verdeckung der Sonotrodenspitze durch Kavitationsblasen nicht auswerten lässt. Ein Problem stellen allerdings anhaftende Partikel dar, die der Sonotrode teilweise nicht konstant folgen und die gemessene Auslenkung so scheinbar erhöhen oder verringern können. Es wurde versucht, dies durch Wahl eines geeigneten Bildstreifens zu reduzieren. In Abbildung 4.8 ist zu erkennen, dass die Auslenkung der Sonotrode bei $30^{\circ} \mathrm{C}$ systematisch höher liegt als bei 10 und $20^{\circ} \mathrm{C}$. Dies liegt eventuell an einer Verschiebung der Hauptresonanz der Sonotrode mit der Temperatur. Aus diesem Grund lassen sich die Messungen bei den unterschiedlichen Temperaturen nicht direkt miteinander vergleichen, da für alle Messungen die gleiche Frequenz verwendet und nicht entsprechend der Resonanzverschiebung nachgeregelt wurde.

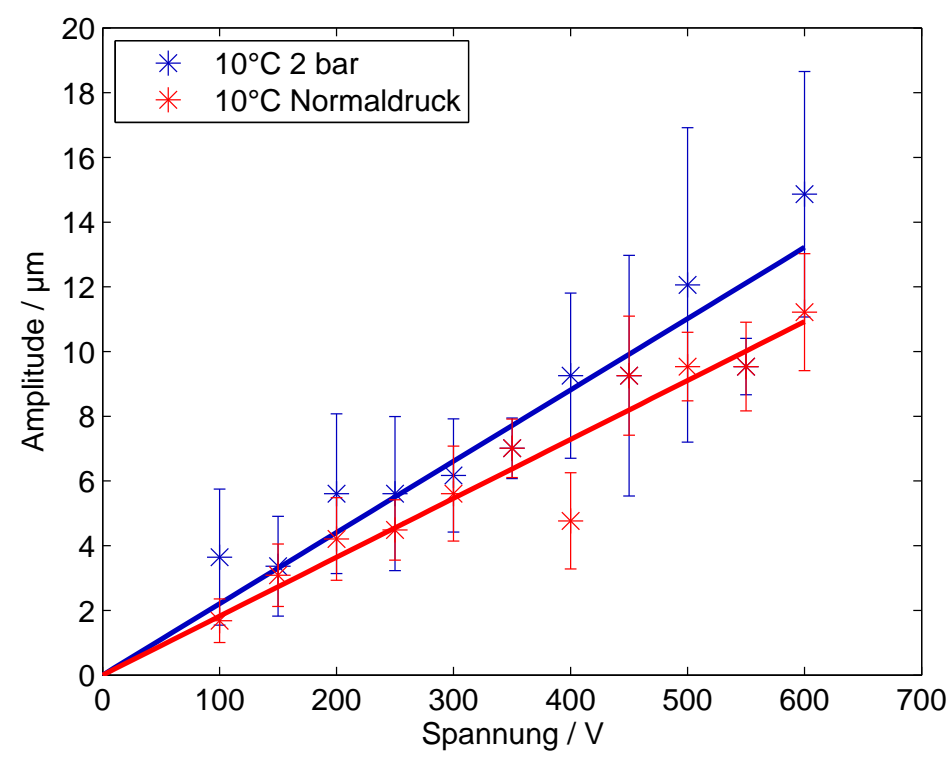

Abbildung 4.3.: Die Auslenkungsamplitude der Sonotrode aufgetragen über der anliegenden Spannung bei $10 \pm 2{ }^{\circ} \mathrm{C}$ und $17.281 \pm 0.001 \mathrm{kHz}$ für Normaldruck und $2.0 \pm 0.5$ bar Überdruck. 


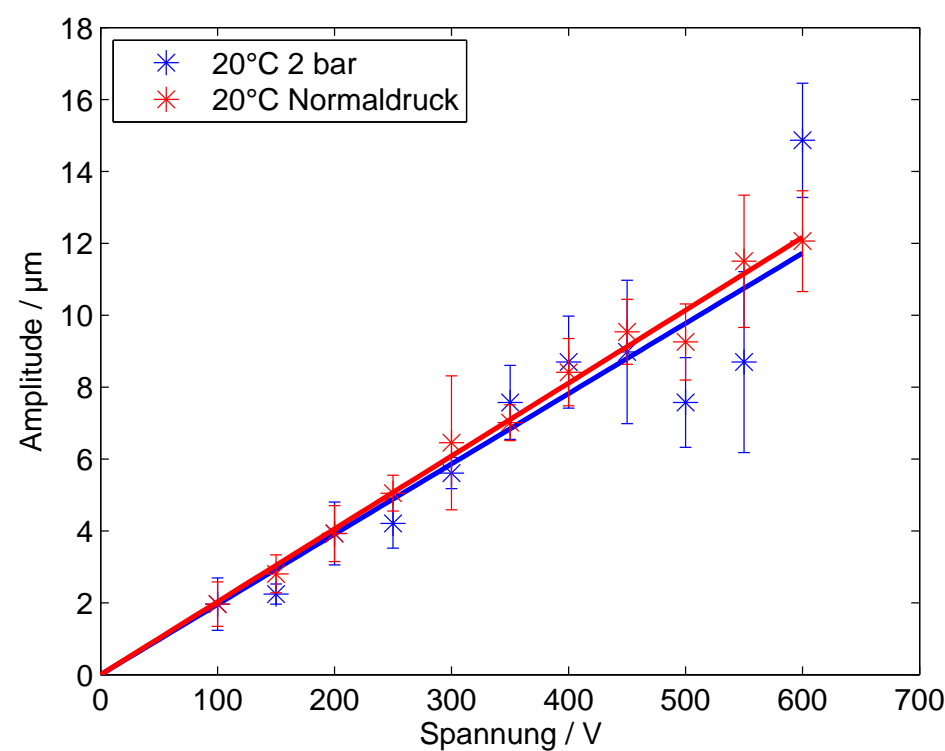

Abbildung 4.4.: Die Auslenkungsamplitude der Sonotrode aufgetragen über der anliegenden Spannung bei $21 \pm 1^{\circ} \mathrm{C}$ und $17.281 \pm 0.001 \mathrm{kHz}$ für Normaldruck und $2.0 \pm 0.5$ bar Überdruck.

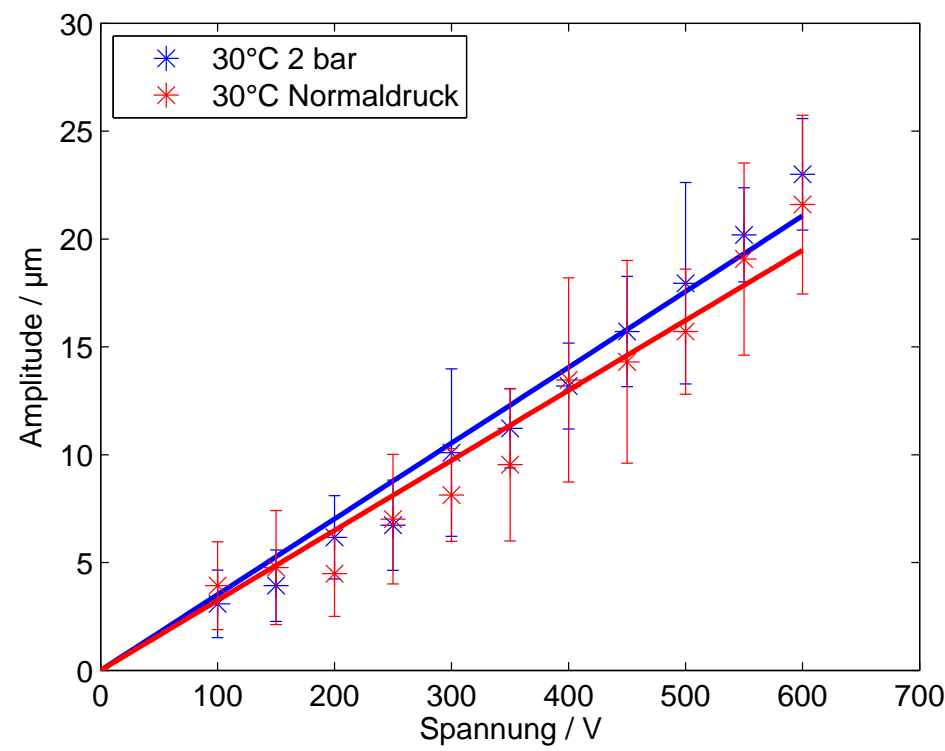

Abbildung 4.5.: Die Auslenkungsamplitude der Sonotrode aufgetragen über der anliegenden Spannung bei $30 \pm 1^{\circ} \mathrm{C}$ und $17.281 \pm 0.001 \mathrm{kHz}$ für Normaldruck und $2.0 \pm 0.5$ bar Überdruck. 


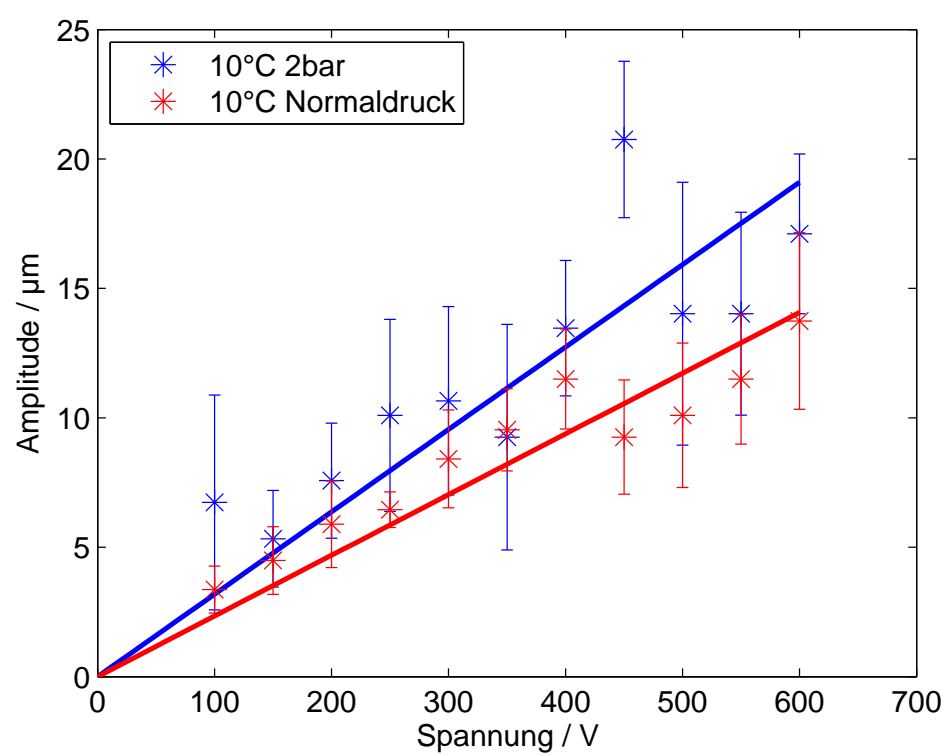

Abbildung 4.6.: Die Auslenkungsamplitude der Sonotrode aufgetragen über der anliegenden Spannung bei $10 \pm 2{ }^{\circ} \mathrm{C}$ und $17.381 \pm 0.001 \mathrm{kHz}$ für Normaldruck und $2.0 \pm 0.5$ bar Überdruck.

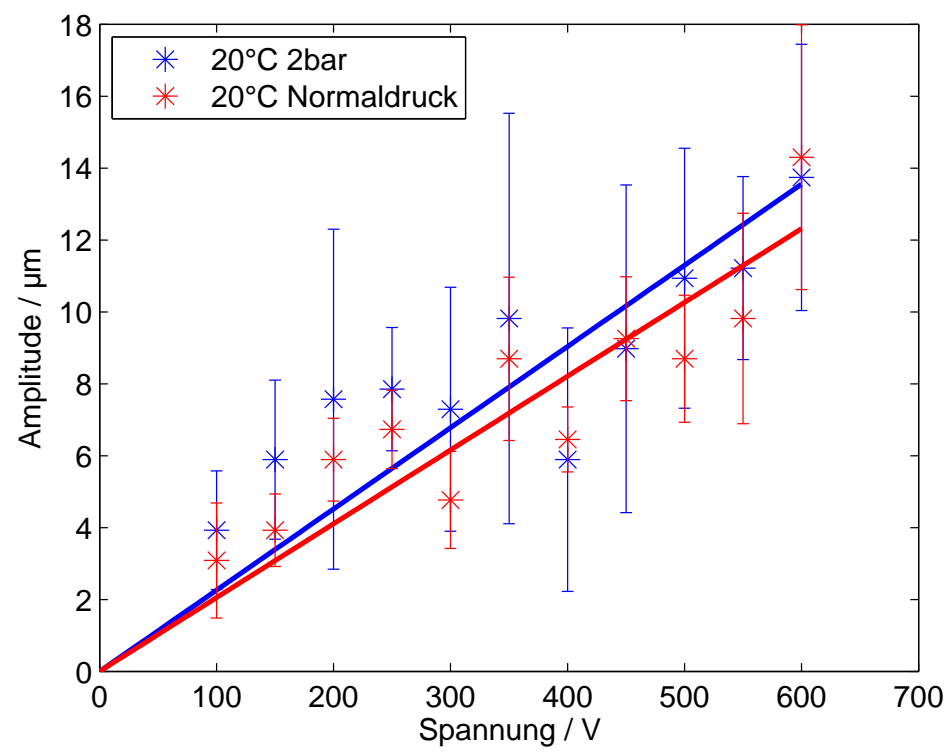

Abbildung 4.7.: Die Auslenkungsamplitude der Sonotrode aufgetragen über der anliegenden Spannung bei $24 \pm 1{ }^{\circ} \mathrm{C}$ und $17.381 \pm 0.001 \mathrm{kHz}$ für Normaldruck und $2.0 \pm 0.5$ bar Überdruck. 


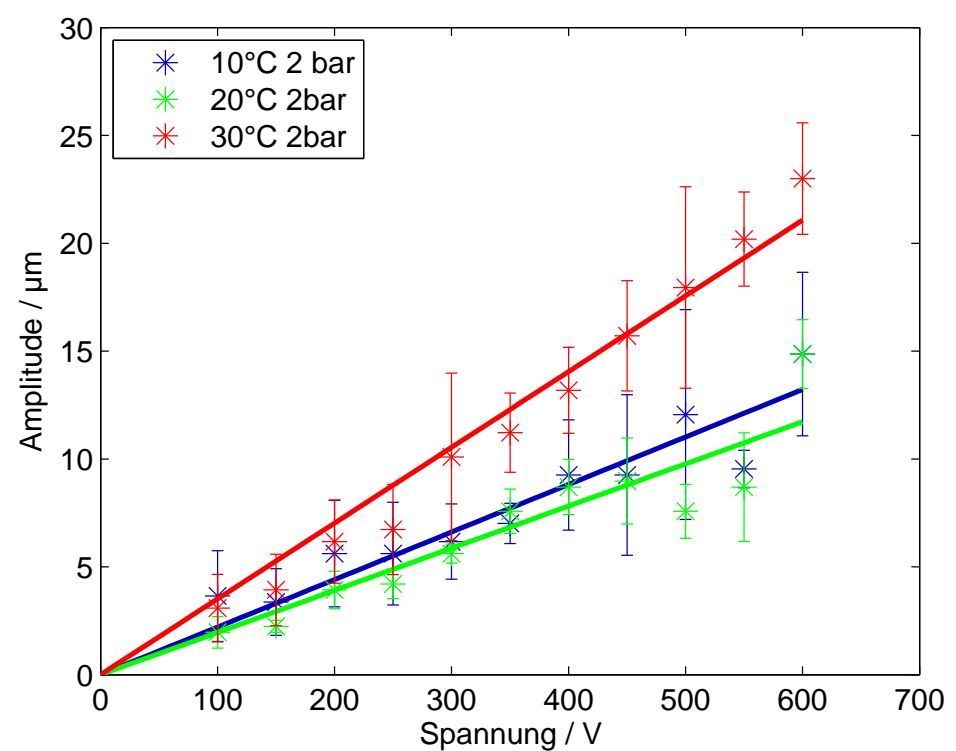

Abbildung 4.8.: Die Auslenkungsamplitude der Sonotrode aufgetragen über der anliegenden Spannung bei verschiedenen Temperaturen und $17.281 \pm 0.001 \mathrm{kHz}$ für $2.0 \pm 0.5$ bar Überdruck. 


\subsection{Vergleich der Kavitationsblasenfelder bei Pa- rametervariation}

Anhand von Beispielen wird im folgenden Kapitel gezeigt, wie sich die Blasenwolke unter der Sonotrode bei Parametervariation verändert. Hierzu wurden aus einigen Durchlichtaufnahmen Bilder mit maximal aufgeschwungenen Blasen ausgewählt. Um die Blasenwolke besser sichtbar zu machen, wurde ein Hintergrundbild abgezogen.

\subsubsection{Verschiedene Spannungen}

In Abbildung 4.9 sind beispielhaft typische Blasenwolken an der Sonotrode bei verschiedenen Spannungen abgebildet. Es ist zu erkennen, dass die Ausdehnung der Blasenwolke mit zunehmender Spannung zunimmt. Vor allem die Entfernung, bei der noch große Blasen sichtbar sind, verschiebt sich deutlich nach unten. Die sichtbare Blasendichte und seitliche Ausdehnung der Wolke verändern sich zeitlich stark und hängen somit in großen Teilen von der zeitlichen Wahl des Bildes ab. Die Ausdehnung der Blasenwolke resultiert aus einer Wechselwirkung zwischen Schallfeld und Blasendichte. Ein höherer Schalldruck lässt mehr Blasen aufschwingen, die ihrerseits das Schallfeld abschirmen. Dadurch nimmt der Schalldruck in einiger Entfernung vom Wandler im Vergleich zum theoretischen Schalldruck, der sich aus der Auslenkung der Sonotrode berechnen lässt, stark ab [64]. Außerdem kann diese Wechselwirkung zwischen Schalldruck und Blasendichte unter Umständen zu einer Oszillation der Blasendichte führen und ist somit möglicherweise ein weiterer Effekt, der zur Entstehung von subharmonischen Frequenzen in Kavitationsblasenfeldern beiträgt. Auf diesen Effekt wird in Kapitel 4.7.3 näher eingegangen.

\subsubsection{Verschiedene Temperaturen und Gasgehalte}

Die Größe der Blasenwolke an einer Sonotrode hängt sowohl von der Temperatur als auch vom Gasgehalt der Flüssigkeit ab. Bei Erhöhung der Temperatur erhöht sich der Dampfdruck des Wassers und es reißen mehr Blasen auf. Eine Zunahme des Gasgehalt im Wasser hat einen ähnlichen Effekt. Zusätzlich befindet sich nichtkondensierbares Gas in der Blase, die Kavitationsschwelle sinkt und es entstehen mehr Blasen [99]. Außerdem sorgen beide Effekte für einen erhöhten Gasdruck in der Blase, was die Blasen größer aufschwingen lässt. Abbildungen 4.10 und 4.11 zeigen Beispiele der maximalen Betriebsspannung von $600 \mathrm{~V}$ bei verschiedenen Gasgehalten und unterschiedlichen Temperaturen. Um 


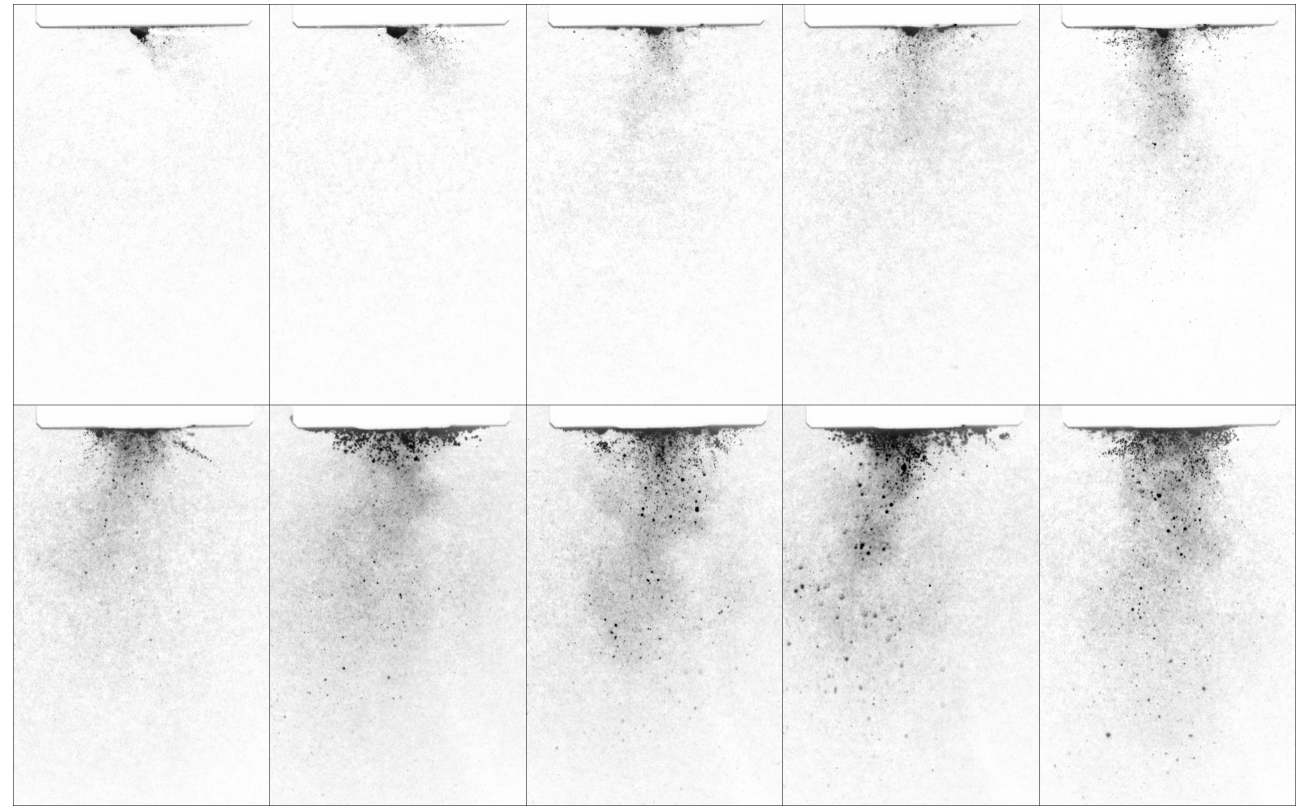

\begin{abstract}
Abbildung 4.9.: Vergleich der Blasenstrukturen bei verschiedenen Spannungen bei $17.281 \mathrm{kHz}$ Anregungsfrequenz. Links oben $150 \mathrm{~V}$ bis rechts unten in $50 \mathrm{~V}$-Schritten $600 \mathrm{~V}_{\mathrm{pp}}$. Alle wurden bei $30^{\circ} \mathrm{C}$ und $90 \%$ Gasgehalt aufgenommen. Die Aufnahmerate betrug 5000 Bps mit etwa 2 us Blitzdauer der LED. Die Spitze der Sonotrode mit $1 \mathrm{~cm}$ Durchmesser ist im oberen Bereich in weiß zu erkennen. In schwarz sind größere Blasen und als grauer Schleier kleinere Blasen zu erkennen.
\end{abstract}

eine möglichst gute Vergleichbarkeit zu gewährleisten wurden Bilder ausgewählt, bei denen die Blasen an der Sonotrode maximal aufgeschwungen sind. In beiden Abbildungen wurde der Hintergrund (ein Bild bei 2 bar ohne Kavitation) abgezogen, um die Blasen deutlicher sichtbar zu machen. In Abbildung 4.10 wurden Helligkeit und Kontrast so angepasst, dass die Blasenwolke möglichst gut sichtbar ist. In Abbildung 4.11 wurde hingegen bei jedem Einzelbild der gleiche Schwellwert von 245 angelegt (die Grauwerte sind auf einer Skala von 0 (weiß) bis 255 (schwarz) angegeben). Die oben beschriebenen Effekte sind in den Abbildungen 4.10 und 4.11 gut zu erkennen. Mit zunehmendem Gasgehalt nimmt die Größe (Breite und Reichweite) der Blasenwolke deutlich zu. Auch die aktive Fläche an der Sonotrode (Fläche an der Sonotrode anhaftender Blasen) wird größer. Mit steigender Temperatur ist deutlich zu erkennen, dass die Blasengröße zunimmt. Vor allem sind größere Blasen in immer größerer Entfernung von der Sonotrode auszumachen. Die Größe der Blasenwolke nimmt bei Erhöhung der Temperatur weniger stark zu als bei Erhöhung des Gasgehaltes. 

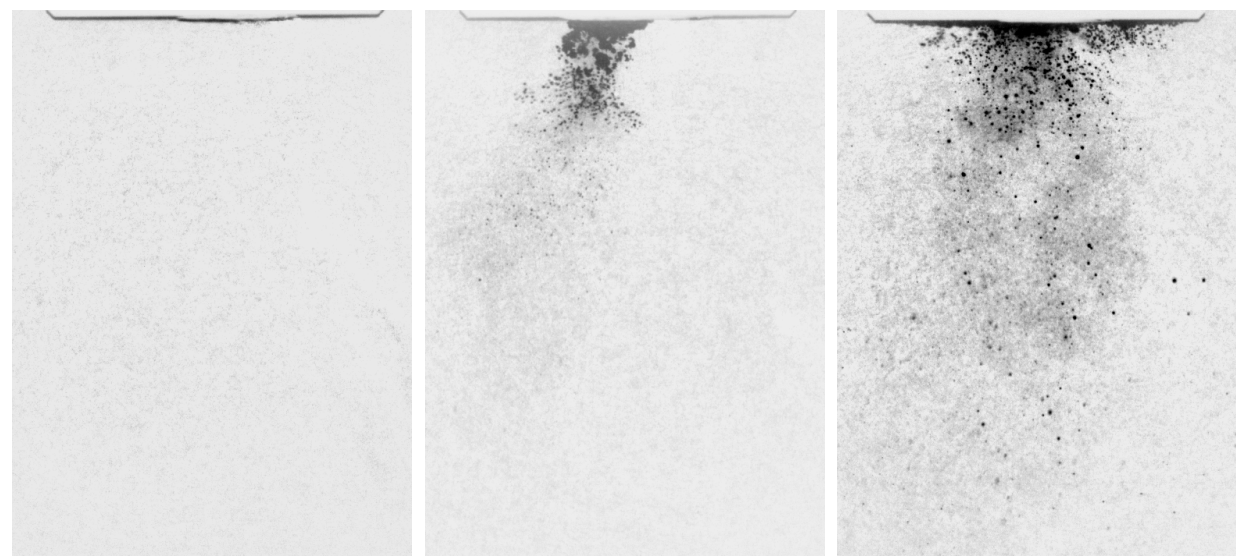

a) $10{ }^{\circ} \mathrm{C}, 5 \% \mathrm{Gas}$

b) $10{ }^{\circ} \mathrm{C}, 50 \% \mathrm{Gas}$

c) $10{ }^{\circ} \mathrm{C}, 90 \% \mathrm{Gas}$
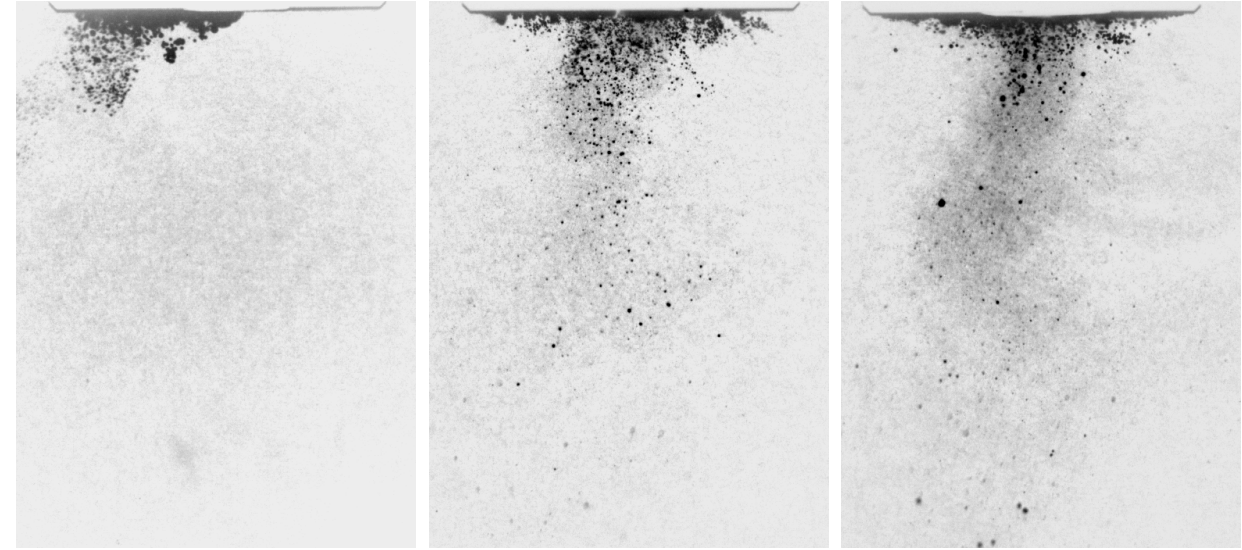

d) $20^{\circ} \mathrm{C}, 5 \% \mathrm{Gas}$

e) $20^{\circ} \mathrm{C}, 50 \%$ Gas

f) $20^{\circ} \mathrm{C}, 90 \%$ Gas
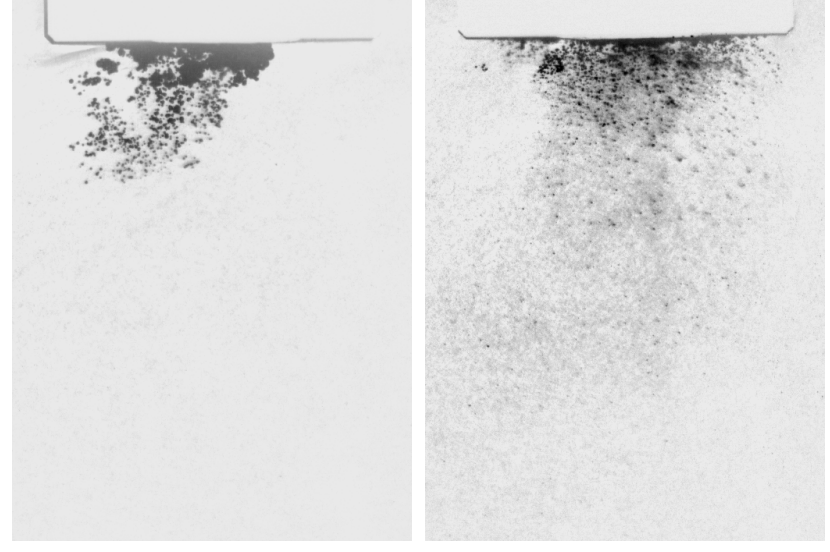

g) $30^{\circ} \mathrm{C}, 5 \%$ Gas

h) $30{ }^{\circ} \mathrm{C}, 50 \%$ Gas

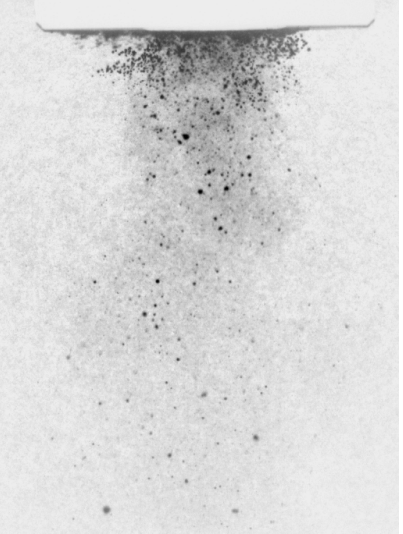

i) $30{ }^{\circ} \mathrm{C}, 90 \%$ Gas

Abbildung 4.10.: Blasenwolken an der Sonotrode bei verschiedenen Temperaturen und Gasgehalten. Die Anzahl der Blasen nimmt mit zunehmendem Gasgehalt zu. Mit Erhöhung der Temperatur werden die Blasen hauptsächlich größer. 

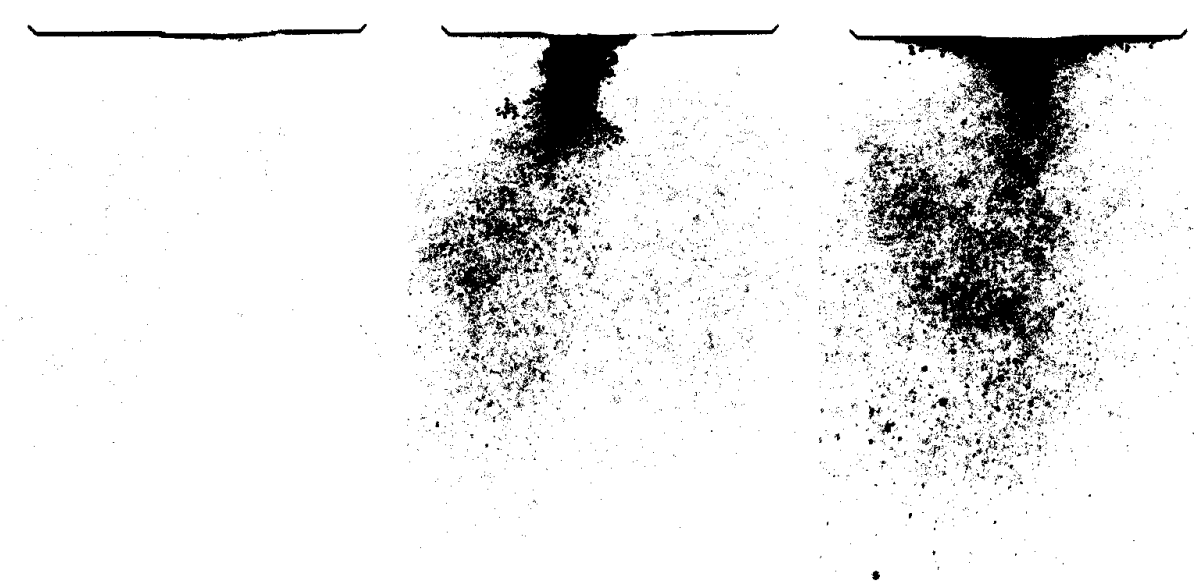

a) $10{ }^{\circ} \mathrm{C}, 5 \%$ Gas

b) $10{ }^{\circ} \mathrm{C}, 50 \%$ Gas

c) $10{ }^{\circ} \mathrm{C}, 90 \%$ Gas

d) $20^{\circ} \mathrm{C}, 5 \% \mathrm{Gas}$
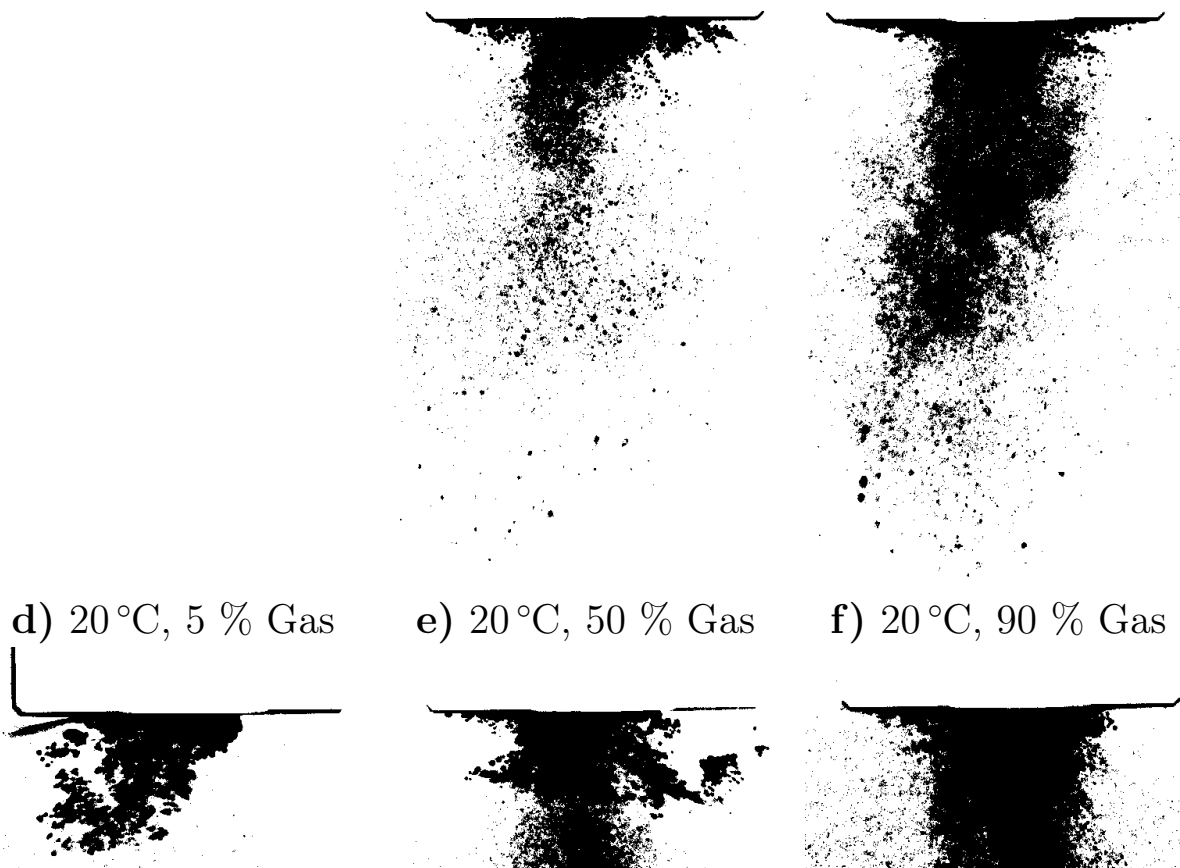

e) $20^{\circ} \mathrm{C}, 50 \% \mathrm{Gas}$

f) $20{ }^{\circ} \mathrm{C}, 90 \% \mathrm{Gas}$
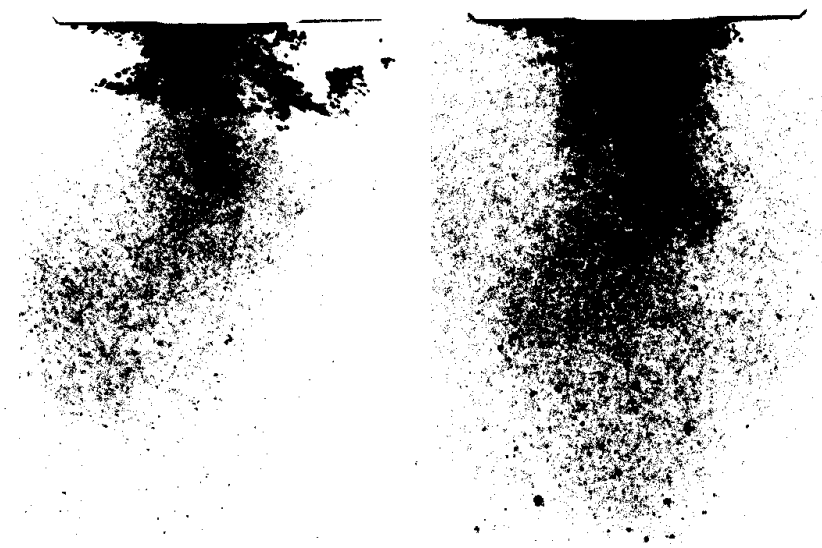

g) $30^{\circ} \mathrm{C}, 5 \%$ Gas

h) $30^{\circ} \mathrm{C}, 50 \%$ Gas

i) $30^{\circ} \mathrm{C}, 90 \%$ Gas

Abbildung 4.11.: Blasenwolken an der Sonotrode bei verschiedenen Temperaturen und Gasgehalten. Die Anzahl der Blasen nimmt mit zunehmendem Gasgehalt zu. Mit Erhöhung der Temperatur werden die Blasen hauptsächlich größer. Für $20^{\circ} \mathrm{C}$ und $5 \%$ Gasgehalt (Teilabbildung $4.11(\mathrm{~d})$ ) ist keine geeignete Aufnahme vorhanden. 


\subsection{Strömungsfeld bei 2 bar Überdruck}

In den Abbildungen 4.12 bis 4.20 ist das Strömungsfeld an der Sonotrode bei verschiedenen Temperaturen und Gasgehalten in Abhängigkeit von der an der Sonotrode anliegenden Spannung gezeigt (siehe auch [93]). Die Sonotrode ist im oberen Bereich der Bilder fast über die gesamte Bildbreite und etwas nach rechts verschoben in den meisten Bildern als grauer Bereich sichtbar. Um das Geschwindigkeitsfeld zu glätten und fehlerhafte Vektoren zu eliminieren, wurde an jedem Punkt eine Medianfilterung über eine $3 \times 3$-Matrix durchgeführt. Anschließend wurde über die Zeit gemittelt und des Geschwindigkeitsfeld wurde farbcodiert dargestellt. Der Wertebereich der Farbskala ist so gewählt, dass sie durch die Geschwindigkeiten über alle Spannungen voll ausgesteuert wird. In jedem Bild ist die maximal vorkommende Geschwindigkeit geschrieben, um diese besser vergleichen zu können. Die Richtung der Strömung wird durch eingezeichnete Pfeile verdeutlicht. Hierzu wurde der Wertebereich der Pfeillänge gestaucht, um auch in Bereichen geringer Strömung die Richtung erkennen zu können. Die im Auswertungsprogramm PIVLab eingezeichneten Masken sind grau dargestellt. Diese werden in den Daten als „NaN“-Werte gespeichert. Da diese Werte auch auftreten, wenn keine oder zu wenige Partikel vorhanden sind, die Software also keine Korrelationen findet, tauchen auch im resultierenden Geschwindigkeitsfeld teilweise graue Regionen auf.

\section{Strömungen bei $10^{\circ} \mathrm{C}$}

Bei Betrachtung des Geschwindigkeitsfeldes an der Sonotrode bei $10{ }^{\circ} \mathrm{C}$ und $5 \%$ Gasgehalt in Abbildung 4.12 sind deutliche Zunahmen der Geschwindigkeit und der Größe des Geschwindigkeitsfeldes mit der an der Sonotrode anliegenden Spannung zu erkennen. Die angegebenen Maximalgeschwindigkeiten zeigen, dass sich die Geschwindigkeit der Strömung bei 200 und $250 \mathrm{~V}$ noch im Bereich des Rauschens, das heißst im Bereich der noch vorhandenen Restströmung durch das vorherige Aufwirbeln der Partikel (siehe Kapitel 4.1), bewegt. Die maximale Geschwindigkeit ist hier noch sehr gering und unabhängig von der Betriebsspannung. $\mathrm{Ab} 250 \mathrm{~V}$ ist im Bereich der Sonotrode eine entstehende Strömung zu erkennen. Auffällig ist, dass die Strömung erst auf die Sonotrode zu und erst bei zunehmender Spannung von ihr weggerichtet ist. Die Strömungsvektoren lassen eine sich mit der anliegenden Spannung ausbreitende Grenze des akustischen Strömungsfeldes erkennen. Weiterhin lässt sich beobachten, dass das Strömungsfeld mit zunehmender Spannung, insbesondere ab $550 \mathrm{~V}$, nach rechts gerichtet ist. Die maximal erreichte Strömungsgeschwindigkeit liegt bei $1.2 \mathrm{~cm} \mathrm{~s}^{-1}$. Bei $10^{\circ} \mathrm{C}$ und 

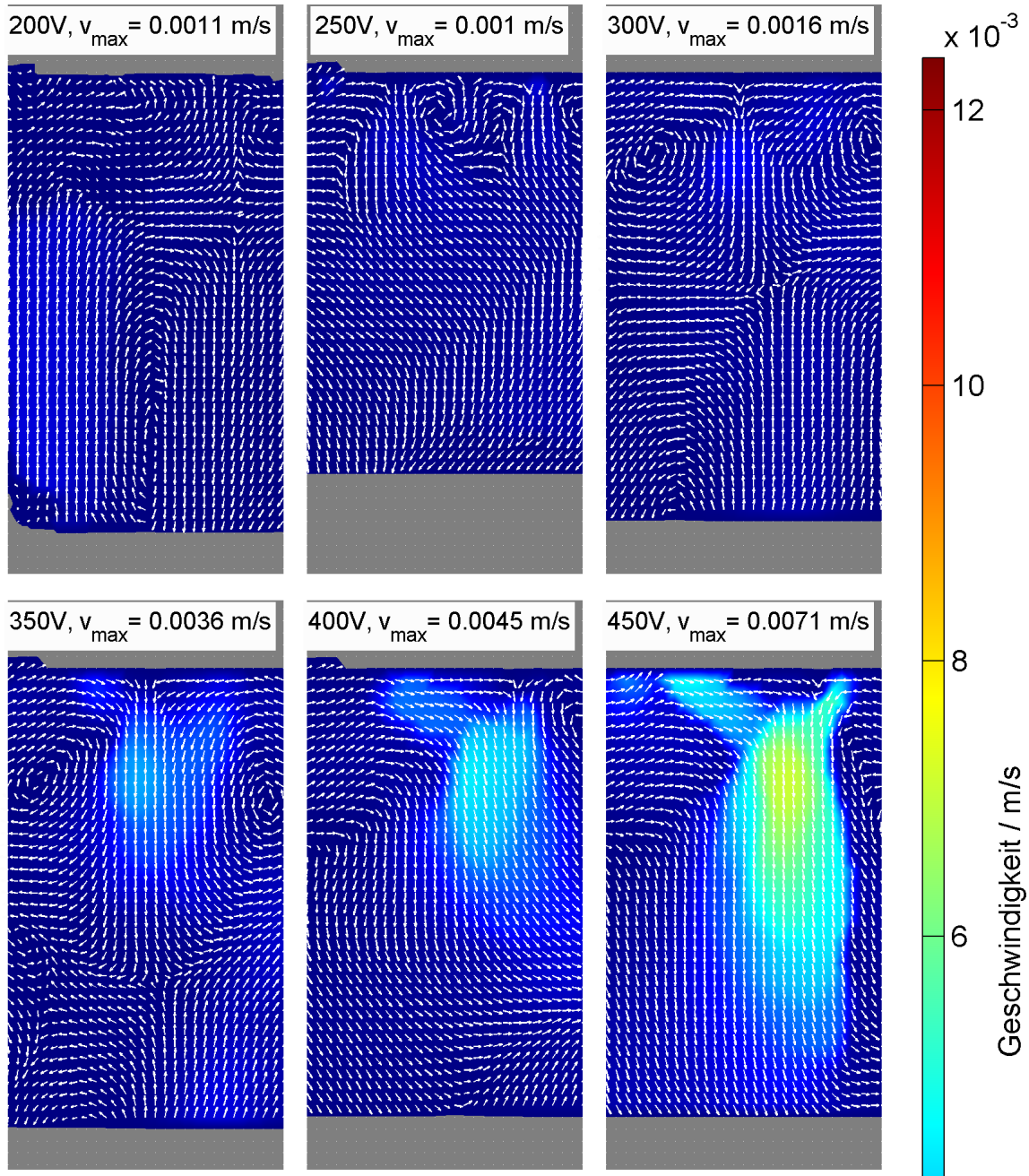

$500 \mathrm{~V}, \mathrm{v}_{\max }=0.0078 \mathrm{~m} / \mathrm{s}$
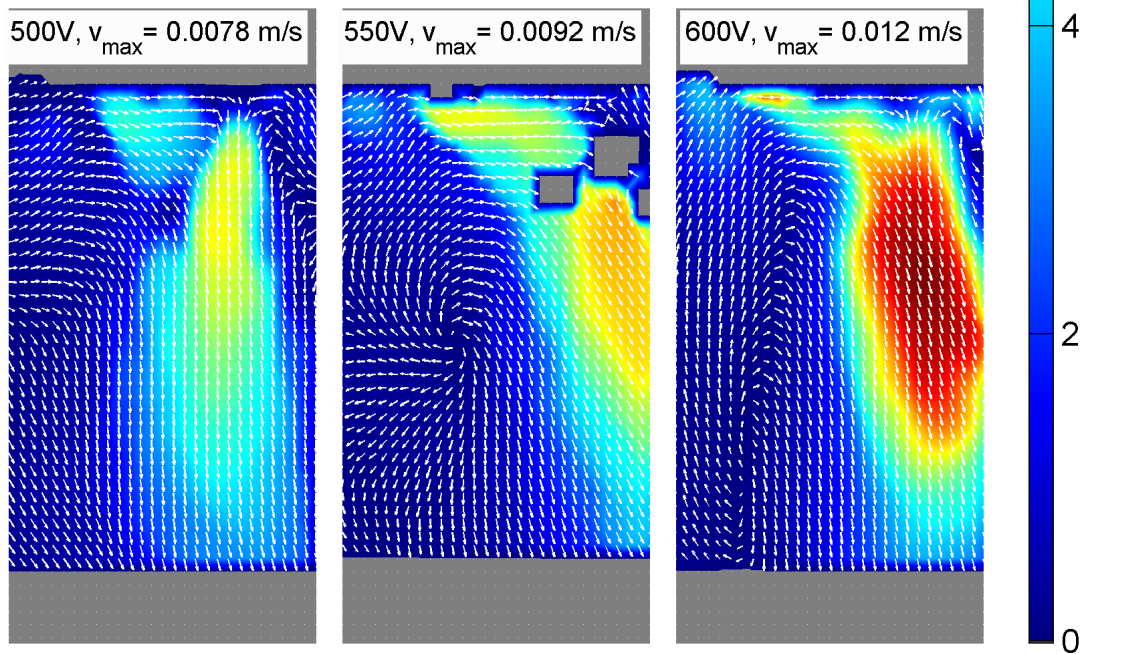

Abbildung 4.12.: Strömungsfeld unter der Sonotrode bei $10^{\circ} \mathrm{C}$ und $5 \%$ Gasgehalt bei 2 bar Überdruck. Die Geschwindigkeit wird durch die Farbe, die Richtung der Strömung durch Pfeile dargestellt. Die grauen Bereiche sind Regionen mit fehlenden Messwerten (Maskierung der Sonotrode, schlechte Beleuchtung oder fehlende Partikel). 

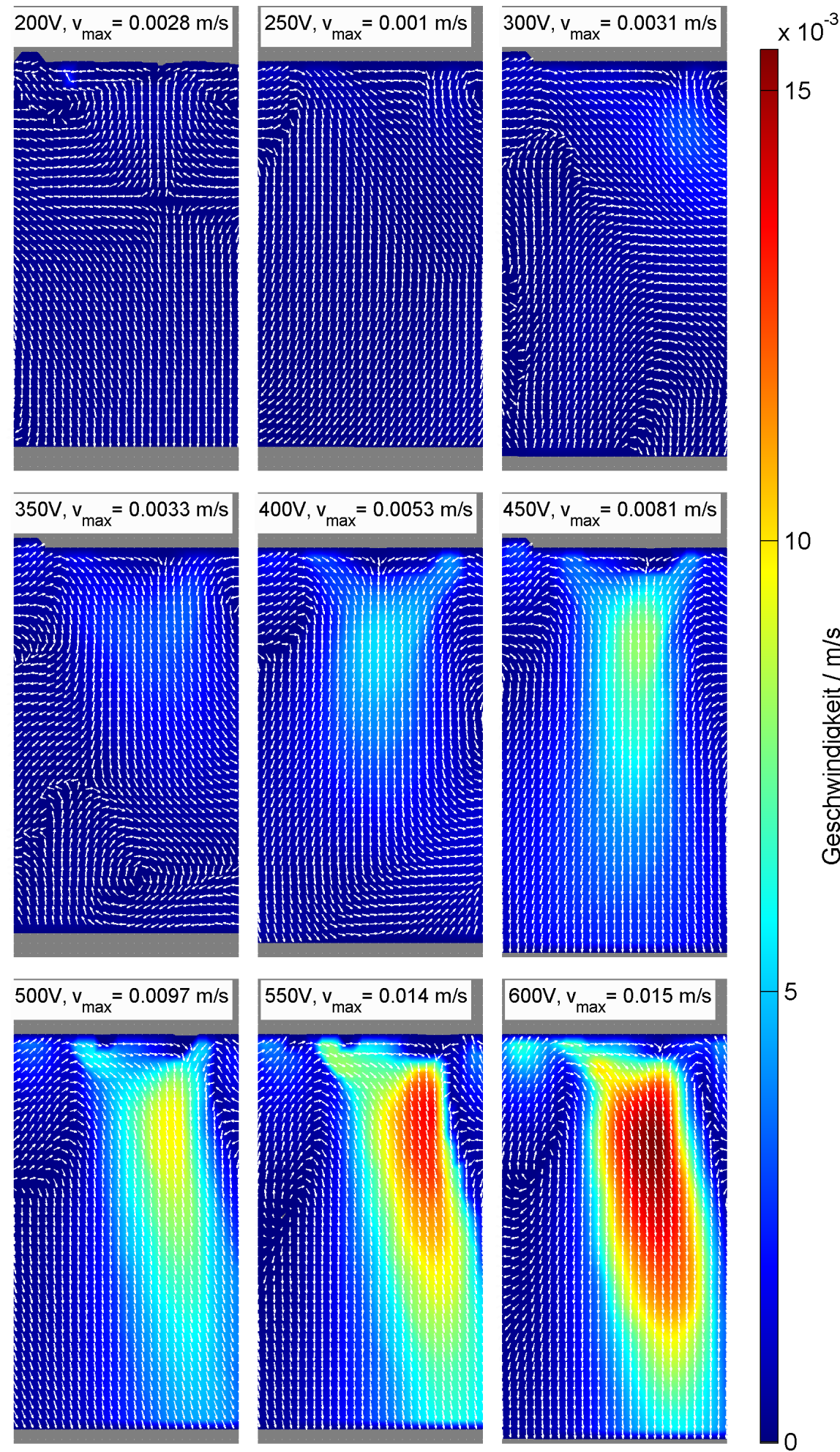

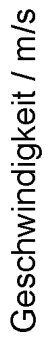
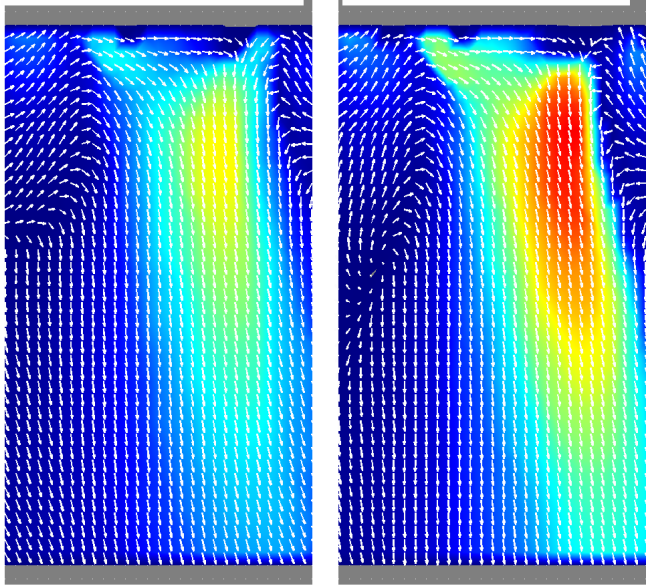

Abbildung 4.13.: Strömungsfeld unter der Sonotrode bei $10^{\circ} \mathrm{C}$ und $50 \%$ Gasgehalt bei 2 bar Überdruck. Die Geschwindigkeit wird durch die Farbe, die Richtung der Strömung durch Pfeile dargestellt. Die grauen Bereiche sind Regionen mit fehlenden Messwerten (Maskierung der Sonotrode, schlechte Beleuchtung oder fehlende Partikel). 
50\% Gasgehalt (siehe Abbildung 4.13) zeigen sich die gleichen Effekte. So nimmt auch hier die Strömung mit der Spannung zu. Bei niedrigen Spannungen ist erst eine Strömung auf die Sonotrode zu zu beobachten. Ab $250 \mathrm{~V}$ ist die Strömung von der Sonotrode weg gerichtet und ihre Grenzen breiten sich mit zunehmender Spannung nach unten aus. Ebenso ist die Strömung bei höheren Spannungen nach rechts gerichtet und die maximale Strömungsgeschwindigkeit liegt bei $1.5 \mathrm{~cm} \mathrm{~s}^{-1}$. Verglichen mit Abbildung 4.13 fällt in Abbildung 4.14 auf, dass die Strömungsfelder von 200 bis $300 \mathrm{~V}$ fast identisch aussehen. Auch hier strömt das Wasser erst auf die Sonotrode zu, dann parallel und erst ab 300 V weg von der Sonotrode. Insgesamt nimmt die maximale Strömungsgeschwindigkeit mit der Spannung zu. Bei $500 \mathrm{~V}$ ist die Maximalgeschwindigkeit geringer als bei $450 \mathrm{~V}$. Das Strömungsfeld wirkt ebenfalls kleiner. Dies könnte an einer Veränderung der Strömungsrichtung senkrecht zur Beobachtungsebene gegenüber der niedrigeren Spannung liegen. Die erreichte Strömungsgeschwindigkeit bei $600 \mathrm{~V}$ beträgt $1.3 \mathrm{~cm} \mathrm{~s}^{-1}$. Insgesamt sind die maximal erreichten Strömungsgeschwindigkeiten bei allen drei Gasgehalten ähnlich. Bei 50\% Gasgehalt liegen sie leicht höher als bei den anderen beiden Messreihen.

\section{Strömungen bei $20^{\circ} \mathrm{C}$}

Bei $20^{\circ} \mathrm{C}$ und $5 \%$ Gasgehalt ist die Vergrößerung des Geschwindigkeitsfeldes von 200 bis $450 \mathrm{~V}$ sichtbar (Abbildung 4.15). So ist der Bereich, in dem durch die Sonotrode eine akustische Strömung induziert wird, durch eine relativ klar erkennbare Linie von Pfeilen abgegrenzt. Die maximale Strömungsgeschwindigkeit wird mit $2.9 \mathrm{~cm} \mathrm{~s}^{-1}$ bei $600 \mathrm{~V}$ erreicht. Auch in Abbildung 4.16 ist die Ausbreitung der Strömungszone zu sehen. Durch Einsetzen von Kavitation bei $450 \mathrm{~V}$ liegen die maximal erreichten Geschwindigkeiten hier allerdings um eine Größenordnung höher als bei $5 \%$ Gasgehalt, bei $600 \mathrm{~V}$ werden $33 \mathrm{~cm} \mathrm{~s}^{-1}$ erreicht. Im Vergleich zu den vorherigen Abbildungen ist $\mathrm{zu}$ sehen, dass die Zone hoher Geschwindigkeit mit Einsetzen der Kavitation vor allem direkt an der Sonotrode schmaler wird. Es wird sehr viel mehr Flüssigkeit von den Seiten in die Mitte der Sonotrode gesaugt und dort nach unten beschleunigt. Bei $600 \mathrm{~V}$ ist direkt unter der Sonotrode ein grauer Bereich zu erkennen. Dies liegt daran, dass in diesem Bereich die Geschwindigkeiten der Tracer-Partikel für die gewählte Auflösung (Fenstergröße 64 Pixel und eine Verschiebung von 16 Pixeln) zu hoch werden und die PIVSoftware somit Fehler produziert. Wegen des hohen Geschwindigkeitsgradienten in diesen Aufnahmen wurde die räumliche Auflösung der PIV-Auswertung als Kompromiss gewählt. Aufgrund des geringen Volumenstroms durch das schmale Strömungsfeld sind die Geschwindigkeiten außerhalb der hohen Strömung sehr 

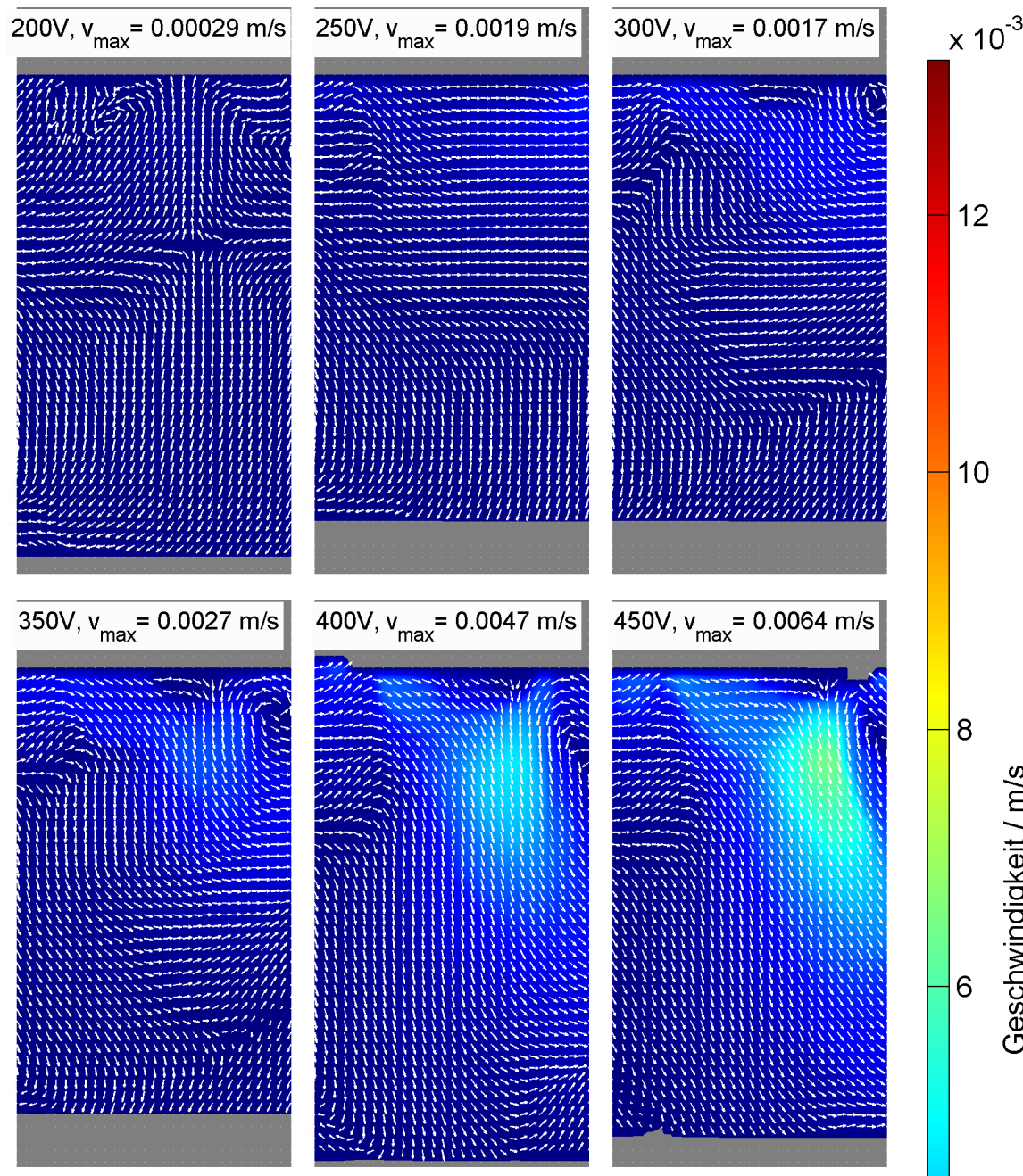

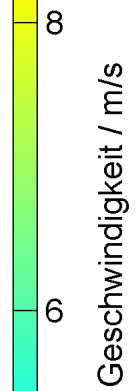
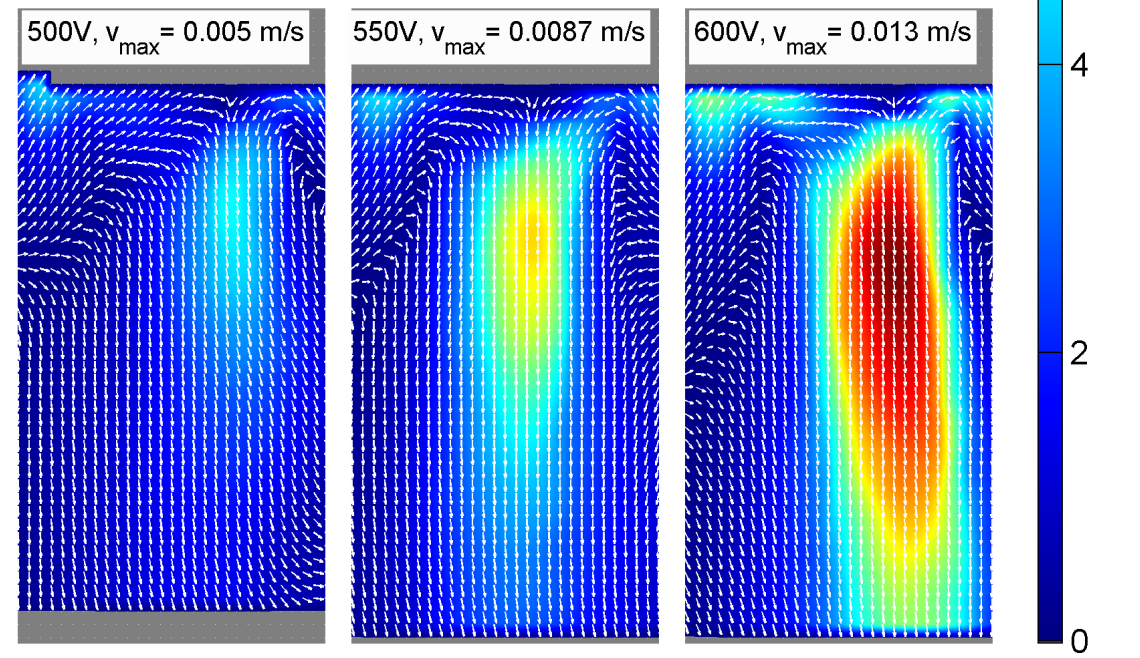

Abbildung 4.14.: Strömungsfeld unter der Sonotrode bei $10^{\circ} \mathrm{C}$ und $90 \%$ Gasgehalt bei 2 bar Überdruck. Die Geschwindigkeit wird durch die Farbe, die Richtung der Strömung durch Pfeile dargestellt. Die grauen Bereiche sind Regionen mit fehlenden Messwerten (Maskierung der Sonotrode, schlechte Beleuchtung oder fehlende Partikel). 

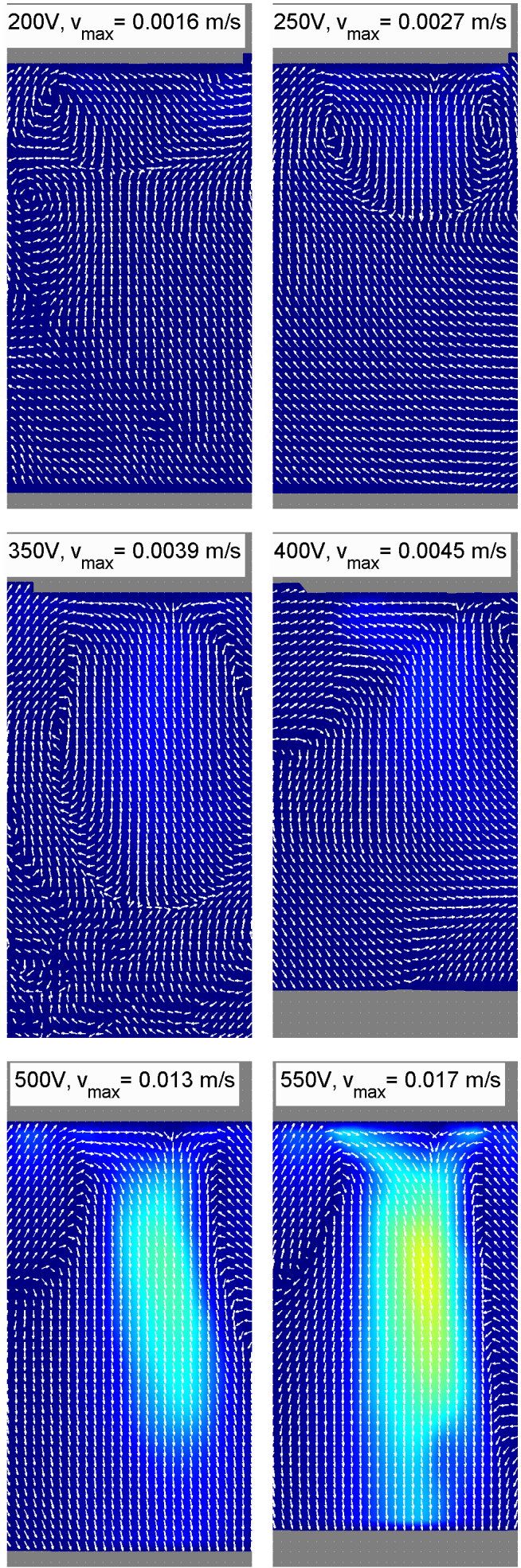

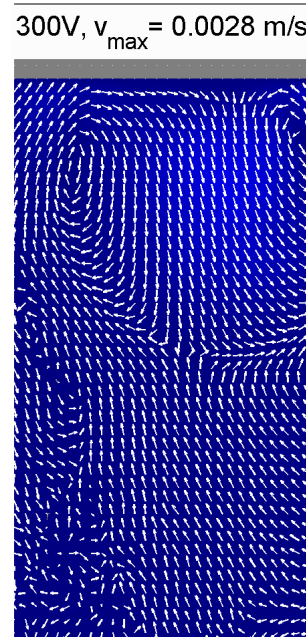

0.025

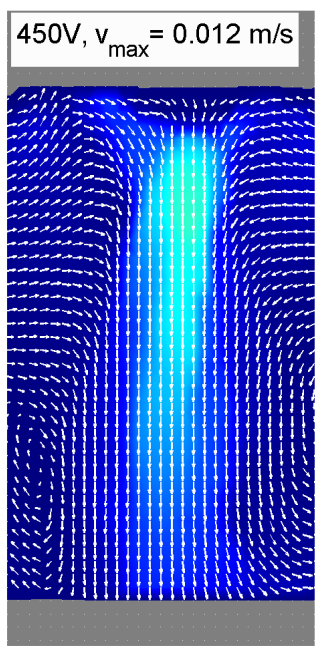

0.02

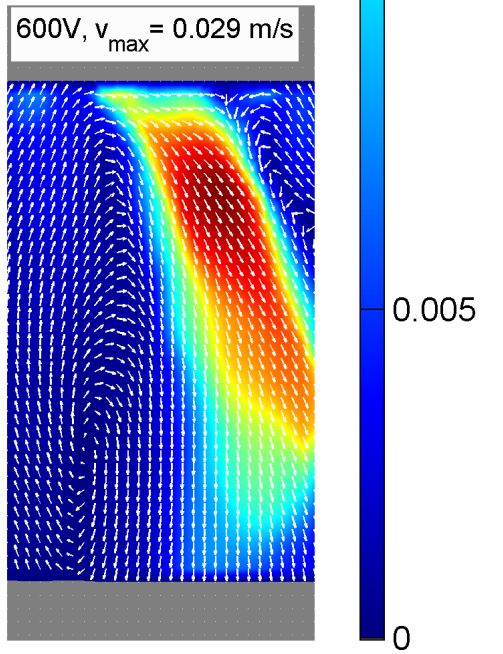

Abbildung 4.15.: Strömungsfeld unter der Sonotrode bei $20^{\circ} \mathrm{C}$ und $5 \%$ Gasgehalt bei 2 bar Überdruck. Die Geschwindigkeit wird durch die Farbe, die Richtung der Strömung durch Pfeile dargestellt. Die grauen Bereiche sind Regionen mit fehlenden Messwerten (Maskierung der Sonotrode, schlechte Beleuchtung oder fehlende Partikel). 
gering, dort würde das Rauschen überwiegen und es wäre keine Auswertung der Geschwindigkeit außerhalb möglich. In Abbildung 4.17 nimmt die maximal erreichte Geschwindigkeit mit der an der Sonotrode anliegenden Spannung monoton zu und erreicht mit $2.8 \mathrm{~cm} \mathrm{~s}^{-1}$ bei $600 \mathrm{~V}$ ihr Maximum. Hier ist ebenfalls zu sehen, dass die Strömungsrichtung für zunehmende Spannung nach rechts kippt.

\section{Strömungen bei $30^{\circ} \mathrm{C}$}

Bei $30^{\circ} \mathrm{C}$ und $5 \%$ Gasgehalt wurden die Geschwindigkeitsfelder ohne Kavitation erst ab $300 \mathrm{~V}$ aufgenommen. Es ist zu erkennen, dass die maximale Strömungsgeschwindigkeit mit der Spannung monoton zunimmt (Abbildung 4.18) und bei 550 und $600 \mathrm{~V}$ mit $1.7 \mathrm{~cm} \mathrm{~s}^{-1}$ ihr Maximum erreicht. Die Ausbreitung des Strömungsfeldes ist in diesem Fall weniger ausgeprägt. Dies liegt daran, dass bei dieser Messreihe das auswertbare Messfenster kleiner war und somit von dem Strömungsfeld schneller ausgefüllt wird. Die Messungen bei 50\% Gasgehalt in Abbildung 4.19 zeigen ebenfalls einen monotonen Anstieg der maximalen Strömungsgeschwindigkeit mit bis zu $2.4 \mathrm{~cm} \mathrm{~s}^{-1}$ bei 600 V. Die Ausbreitung des Strömungsfeldes ist ebenfalls zu erkennen. Allerdings wurden auch hier die Messungen erst ab $300 \mathrm{~V}$ durchgeführt. Die vorhandenen Fehlstellen bei $550 \mathrm{~V}$ kommen durch Fehler der PIV-Software zustande. Dies liegt wahrscheinlich an einer zu geringen Anzahl vorhandener Partikel, so dass durch die Software häufig keine Korrelationen gefunden wurden. In Abbildung 4.20 sind die Strömungsfelder an der Sonotrode bei $90 \%$ Gasgehalt und $30^{\circ} \mathrm{C}$ gezeigt. Die maximale Strömungsgeschwindigkeit nimmt monoton mit der anliegenden Spannung zu. Auch die Ausbreitung des Strömungsfeldes ist zu erkennen. Von 350 auf $400 \mathrm{~V}$ wird die Strömung abrupt schneller und das Strömungsfeld größer. Von 550 auf $600 \mathrm{~V}$ nimmt die maximale Strömungsgeschwindigkeit von 1.9 auf $1.4 \mathrm{~cm} \mathrm{~s}^{-1}$ ab und das Strömungsfeld wird kleiner. Eventuell hat sich bei den Versuchen die kavitierende Fläche an der Sonotrode nach vorne oder hinten verschoben. 

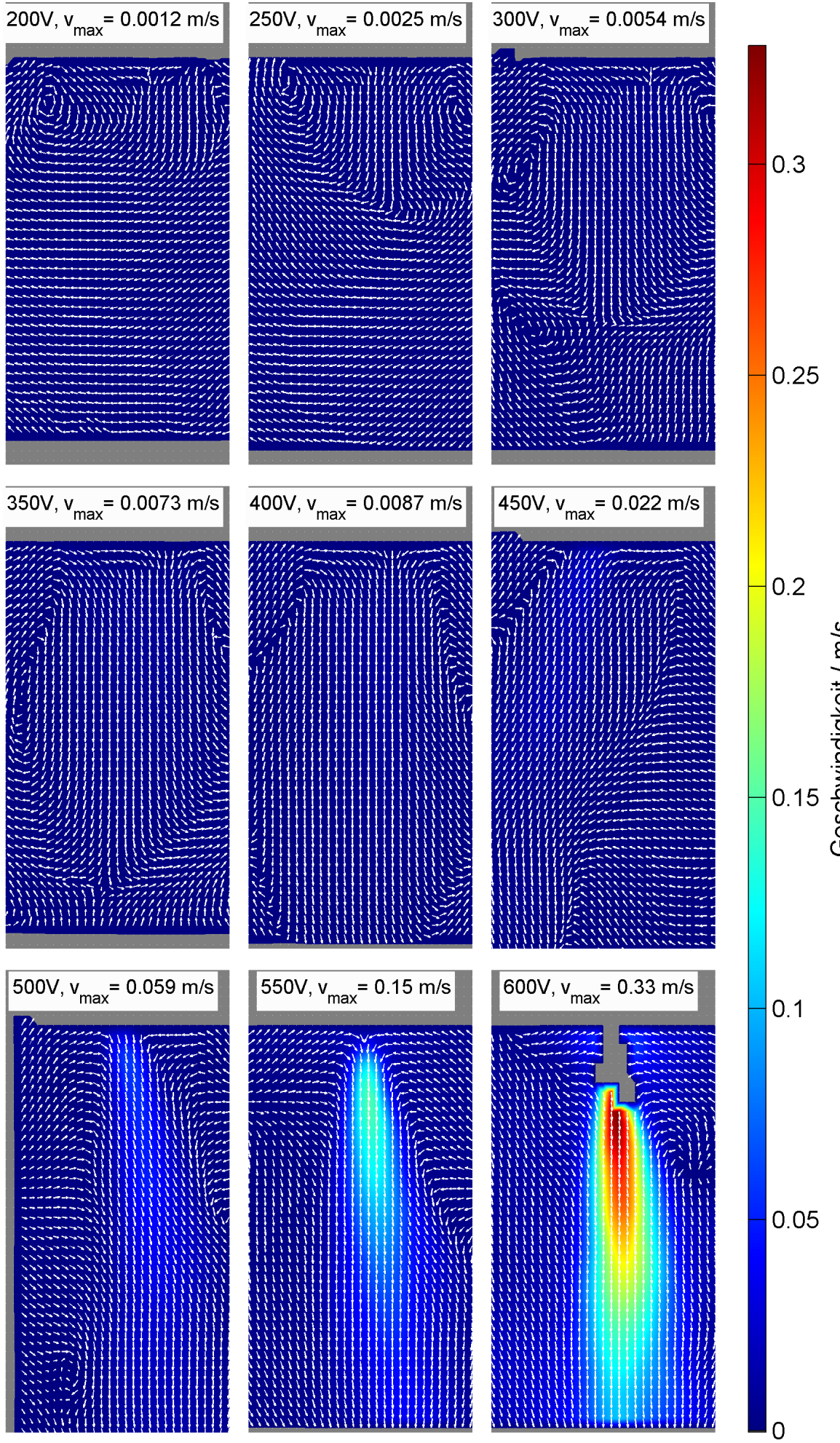

Abbildung 4.16.: Strömungsfeld unter der Sonotrode bei $20^{\circ} \mathrm{C}$ und $50 \%$ Gasgehalt bei 2 bar Überdruck. Die Geschwindigkeit wird durch die Farbe, die Richtung der Strömung durch Pfeile dargestellt. Die grauen Bereiche sind Regionen mit fehlenden Messwerten (Maskierung der Sonotrode, schlechte Beleuchtung oder fehlende Partikel). 

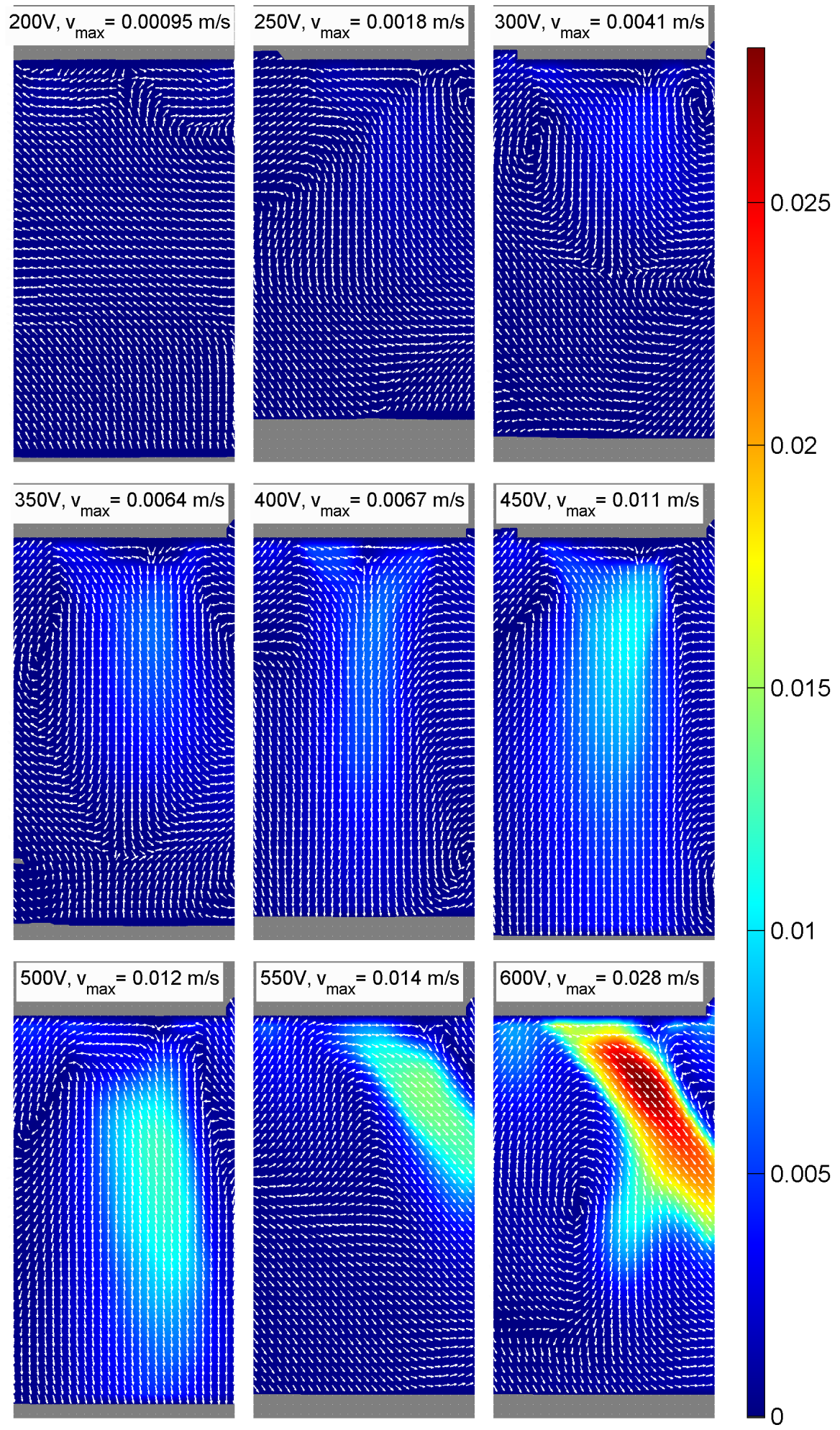

Abbildung 4.17.: Strömungsfeld unter der Sonotrode bei $20^{\circ} \mathrm{C}$ und $90 \%$ Gasgehalt bei 2 bar Überdruck. Die Geschwindigkeit wird durch die Farbe, die Richtung der Strömung durch Pfeile dargestellt. Die grauen Bereiche sind Regionen mit fehlenden Messwerten (Maskierung der Sonotrode, schlechte Beleuchtung oder fehlende Partikel). 

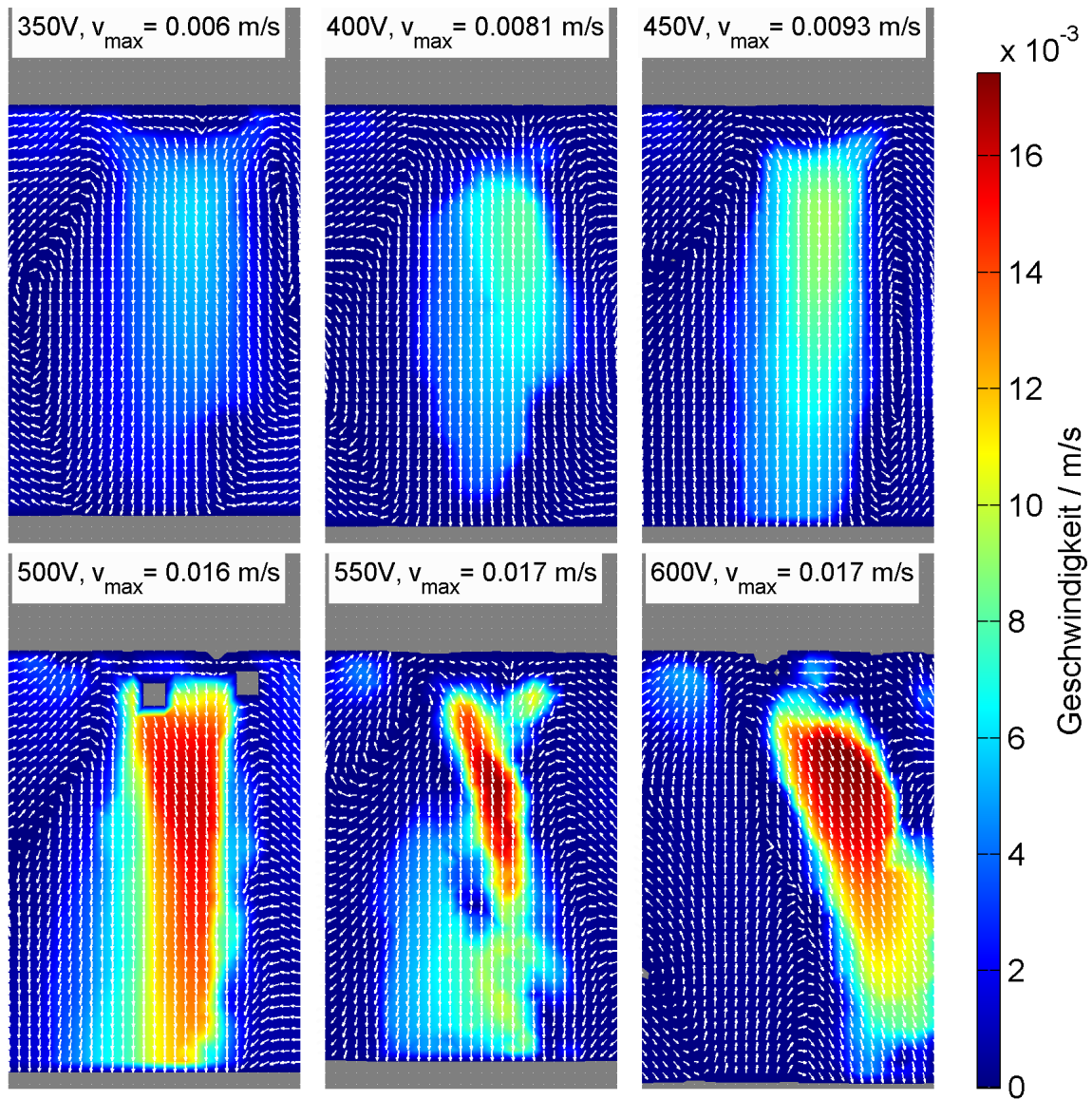

Abbildung 4.18.: Strömungsfeld unter der Sonotrode bei $30^{\circ} \mathrm{C}$ und $5 \%$ Gasgehalt bei 2 bar Überdruck. Die Geschwindigkeit wird durch die Farbe, die Richtung der Strömung durch Pfeile dargestellt. Die grauen Bereiche sind Regionen mit fehlenden Messwerten (Maskierung der Sonotrode, schlechte Beleuchtung oder fehlende Partikel).

\subsection{Strömungsfeld bei Normaldruck}

In den Abbildungen 4.21 bis 4.29 ist das Strömungsfeld an der Sonotrode bei verschiedenen Temperaturen und Gasgehalten in Abhängigkeit von der an der Sonotrode anliegenden Spannung gezeigt (siehe auch [93]). Die Sonotrode im oberen Bereich der Bilder reicht fast über die gesamte Bildbreite und ist etwas nach rechts verschoben. In den meisten Bildern ist die graue Maskierung der Sonotrode aus der PIV-Software zu erkennen. Das Geschwindigkeitsfeld wurde für Strömungsfelder ohne Kavitation über die Zeit gemittelt, aber ungeglättet belassen und farbcodiert dargestellt. Für Strömungsfelder mit Kavitation wurde ein möglichst repräsentatives Bild ausgewählt und ebenfalls ohne Glättung dargestellt. Die Farbskala wurde so angepasst, dass sie durch die Geschwindigkei- 


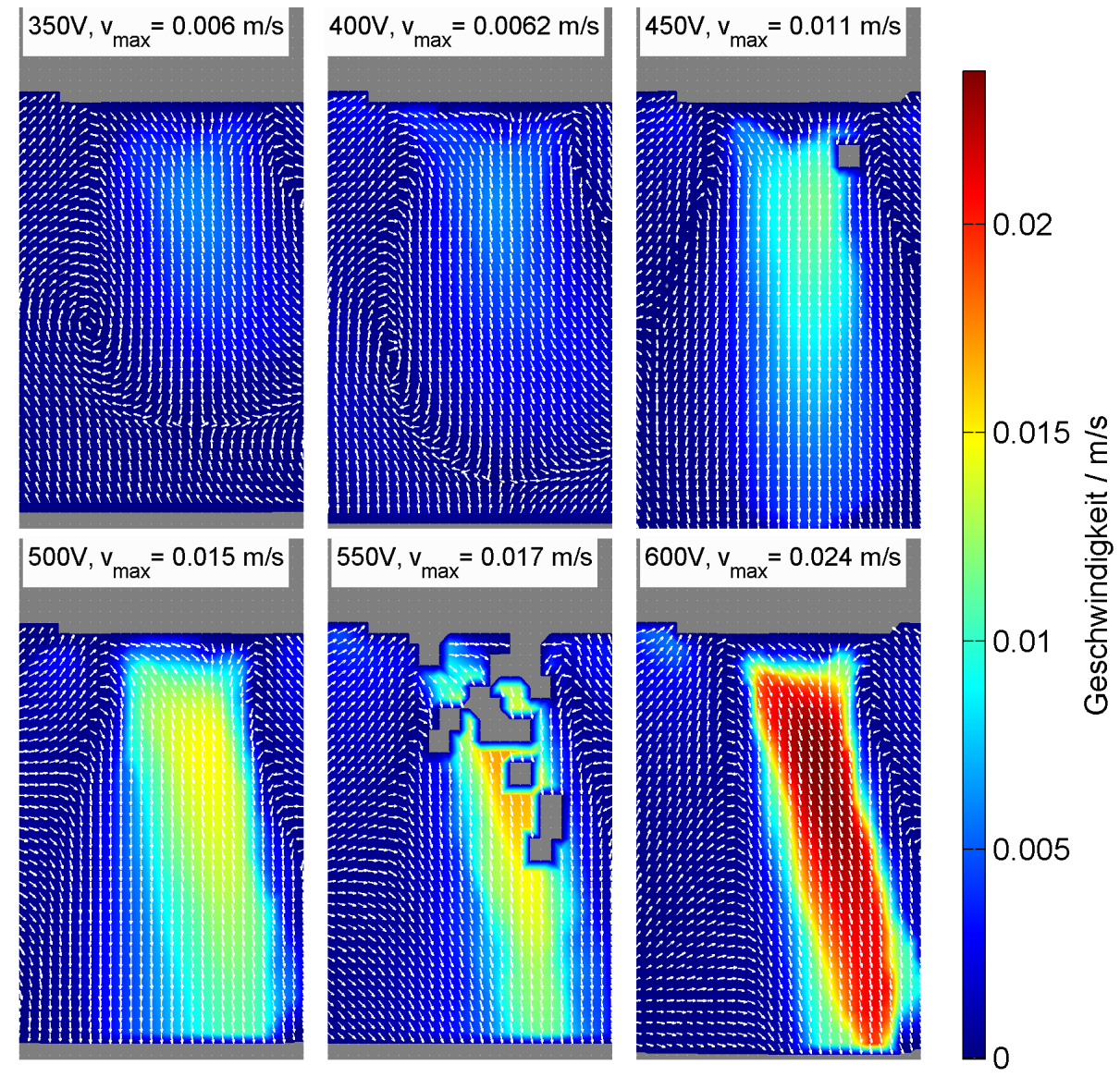

Abbildung 4.19.: Strömungsfeld unter der Sonotrode bei $30^{\circ} \mathrm{C}$ und $50 \%$ Gasgehalt bei 2 bar Überdruck. Die Geschwindigkeit wird durch die Farbe, die Richtung der Strömung durch Pfeile dargestellt. Die grauen Bereiche sind Regionen mit fehlenden Messwerten (Maskierung der Sonotrode, schlechte Beleuchtung oder fehlende Partikel).

ten über alle Spannungen voll ausgesteuert wird. In jedem Bild ist die maximal vorkommende Geschwindigkeit notiert, um diese besser vergleichen zu können. Die Richtung der Strömung wird durch eingezeichnete Pfeile verdeutlicht. Hierzu wurde der Wertebereich der Pfeillänge gestaucht, um auch in Bereichen geringer Strömung die Richtung erkennen zu können. Die im Auswertungsprogramm PIVLab eingezeichneten Masken sind grau dargestellt. Diese werden in den Daten als „NaN“-Werte gespeichert. Da diese Werte auch auftreten, wenn keine oder zu wenige Partikel vorhanden sind, tauchen auch im resultierenden Geschwindigkeitsfeld teilweise graue Regionen auf. 

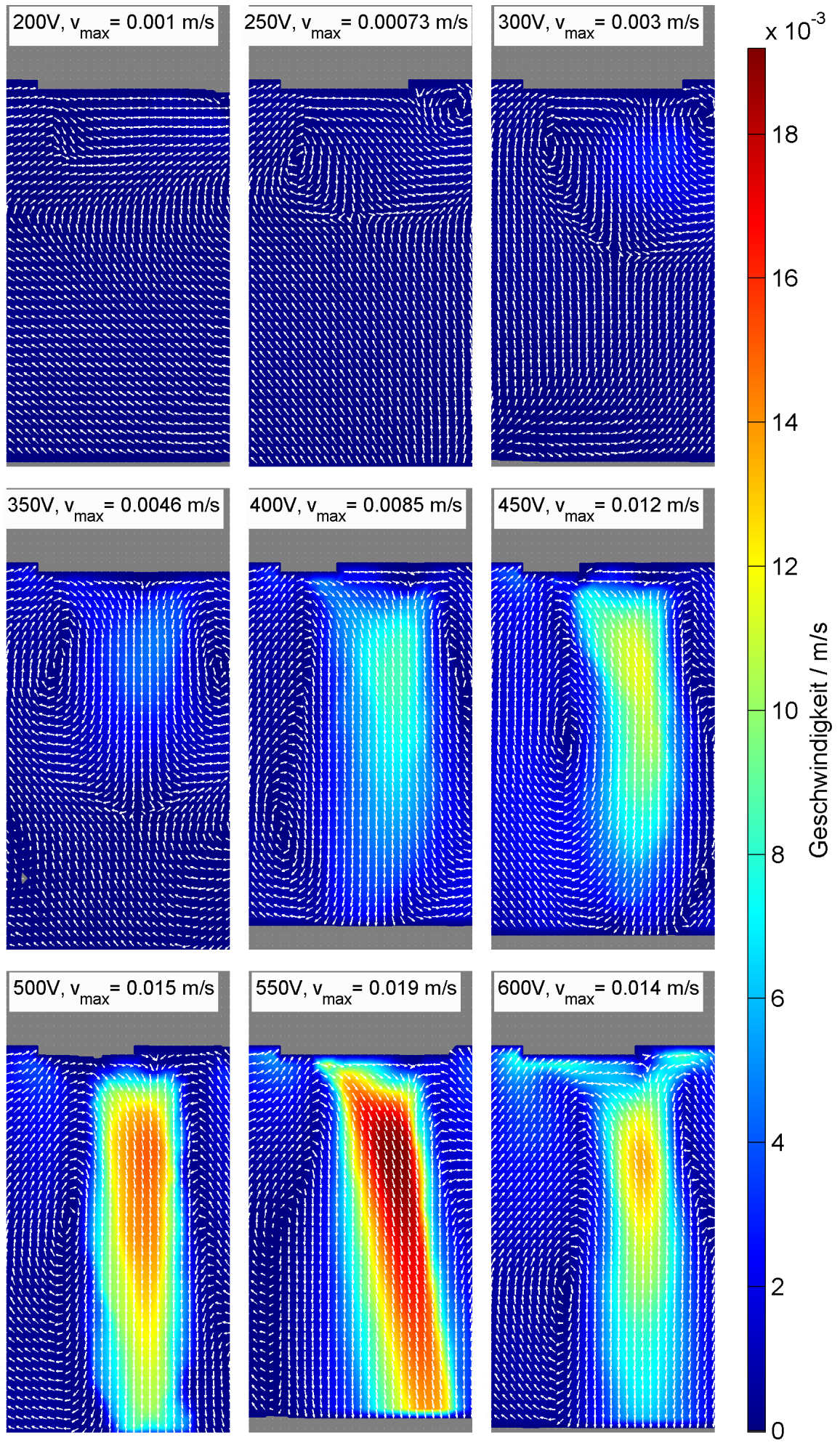

Abbildung 4.20.: Strömungsfeld unter der Sonotrode bei $30^{\circ} \mathrm{C}$ und $90 \%$ Gasgehalt bei 2 bar Überdruck. Die Geschwindigkeit wird durch die Farbe, die Richtung der Strömung durch Pfeile dargestellt. Die grauen Bereiche sind Regionen mit fehlenden Messwerten (Maskierung der Sonotrode, schlechte Beleuchtung oder fehlende Partikel). 


\section{Strömungen bei $10^{\circ} \mathrm{C}$}

In Abbildung 4.21 sind die Strömungsfelder bei $10{ }^{\circ} \mathrm{C}$ und $5 \%$ Gasgehalt dargestellt. In keinem der Fälle trat Kavitation auf. So liegt die maximale Strömungsgeschwindigkeit bei $600 \mathrm{~V}$ bei $8.3 \mathrm{~mm} \mathrm{~s}^{-1}$. Die maximale Strömungsgeschwindigkeit nimmt im Mittel mit der Spannung zu. Bei höheren Spannungen ist das von der Sonotrode weg zeigende Strömungsfeld nach rechts gerichtet. Bei $10{ }^{\circ} \mathrm{C}$ und $50 \%$ Gasgehalt (siehe Abbildung 4.22) setzt die Kavitation bei $350 \mathrm{~V}$ ein. Dies hat eine deutliche Zunahme der maximalen Strömungsgeschwindigkeit zur Folge. Außerdem ist zu erkennen, dass die Varianz der Pfeillänge stark zunimmt. Die Strömungsgeschwindigkeiten innerhalb und außerhalb der Blasenwolke sind stark verschieden. Mit Zunahme der an der Sonotrode anliegenden Spannung wird das Strömungsfeld größer. Die maximale Geschwindigkeit nimmt ebenfalls $\mathrm{zu}$ und erreicht mit $1.6 \mathrm{~m} \mathrm{~s}^{-1}$ bei $600 \mathrm{~V}$ ihr Maximum. Auch außerhalb des von der Sonotrode weg strömenden Bereichs werden die Strömungsgeschwindigkeiten größer und die Pfeile erscheinen gerichteter. Dies liegt daran, dass bei geringen Strömungsgeschwindigkeiten die Aufnahmerate der Kamera zu hoch ist, weshalb die PIV-Software keine gerichtete Verschiebung findet und Rauschen produziert. $\mathrm{Ab}$ einer gewissen Geschwindigkeit ist die Verschiebung pro Bild ausreichend groß und es treten weniger Fehler auf. Die Strömungsfelder bei $10^{\circ} \mathrm{C}$ und $90 \%$ Gasgehalt sind in Abbildung 4.23 dargestellt. Bei diesem Gasgehalt setzt die Kavitation bereits unterhalb von $200 \mathrm{~V}$ ein. Die maximalen Strömungsgeschwindigkeiten nehmen mit Erhöhung der Spannung bis auf $1.5 \mathrm{~m} \mathrm{~s}^{-1} \mathrm{zu}$. Allerdings ist die Zunahme mit der Spannung gering. Innerhalb des Kavitationsblasenfeldes treten auch bei geringen Spannungen schon hohe Strömungsgeschwindigkeiten auf. Auf diesen Zusammenhang wird später noch genauer eingegangen. Die Größe des Strömungsfeldes nimmt allerdings massiv zu. So strömt es bei $200 \mathrm{~V}$ nur in einem kleinen Bereich direkt an der Sonotrode. Bei $600 \mathrm{~V}$ hat sich das Strömungsfeld fast über den gesamten sichtbaren Bildausschnitt ausgebreitet.

\section{Strömungen bei $20^{\circ} \mathrm{C}$}

Bei $20^{\circ} \mathrm{C}$ und $5 \%$ Gasgehalt ist zwischen 200 und $400 \mathrm{~V}$ in Abbildung 4.24 anhand einer Grenzlinie der Strömung zwischen den Pfeilen eine deutliche Vergrößerung des Strömungsfeldes zu erkennen. Die maximale Strömungsgeschwindigkeit nimmt hier monoton zu und erreicht bei $600 \mathrm{~V}$ bis zu $1.9 \mathrm{~m} \mathrm{~s}^{-1}$. Ab $450 \mathrm{~V}$ ist einsetzende Kavitation zu erkennen. Die maximale Geschwindigkeit nimmt stark zu, bleibt dann mit weiter zunehmender Spannung aber ähnlich. Erst bei $600 \mathrm{~V}$ ist wieder eine deutliche Zunahme zu erkennen. Aus den gleichen Gründen, die im vorherigen Abschnitt bereits beschrieben wurden, sind die Pfeile außerhalb des 

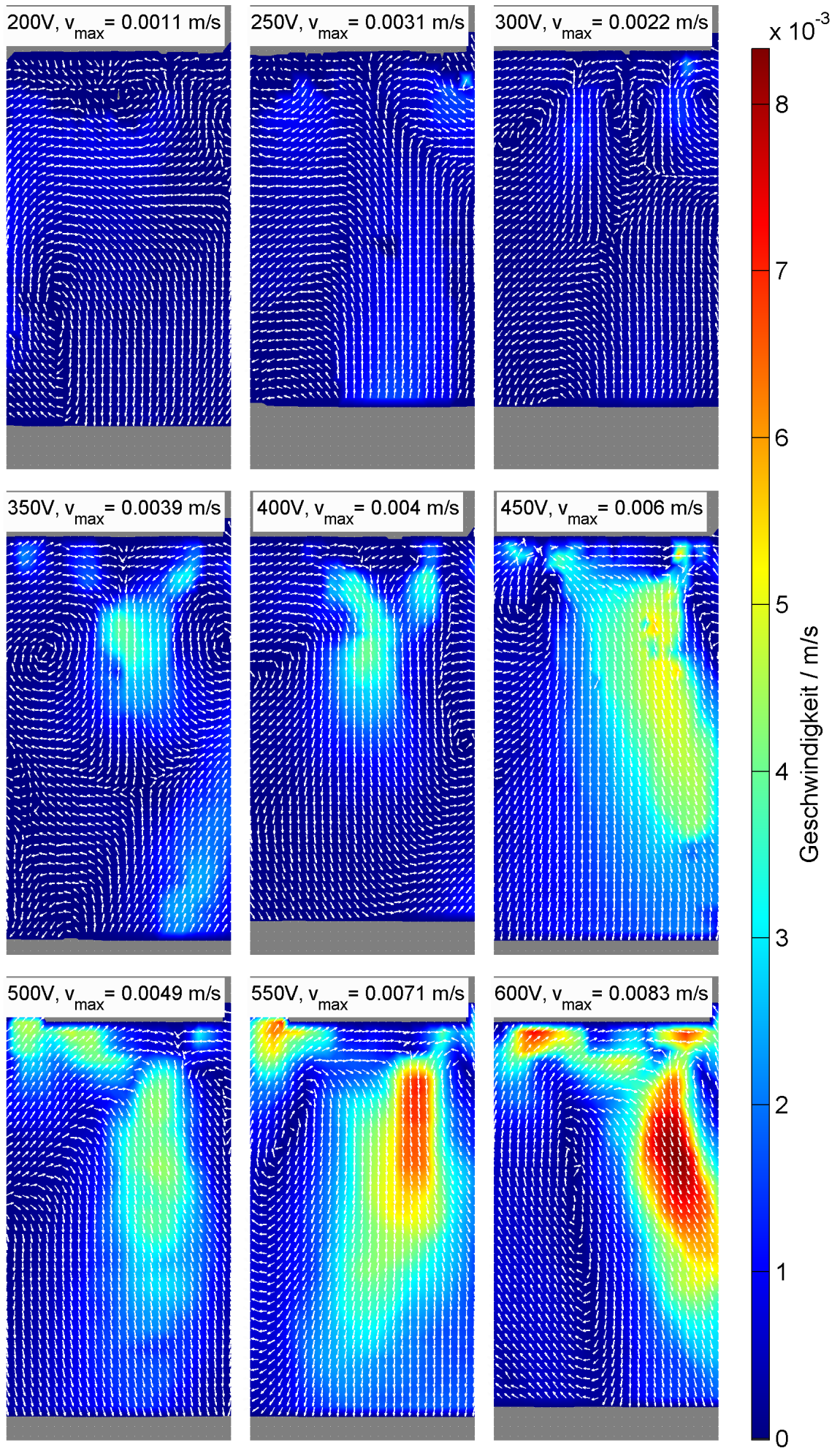

Abbildung 4.21.: Strömungsfeld unter der Sonotrode bei $10^{\circ} \mathrm{C}$ und $5 \%$ Gasgehalt bei Normaldruck. Die Geschwindigkeit wird durch die Farbe, die Richtung der Strömung durch Pfeile dargestellt. Die grauen Bereiche sind Regionen mit fehlenden Messwerten (Maskierung der Sonotrode, schlechte Beleuchtung oder fehlende Partikel). 

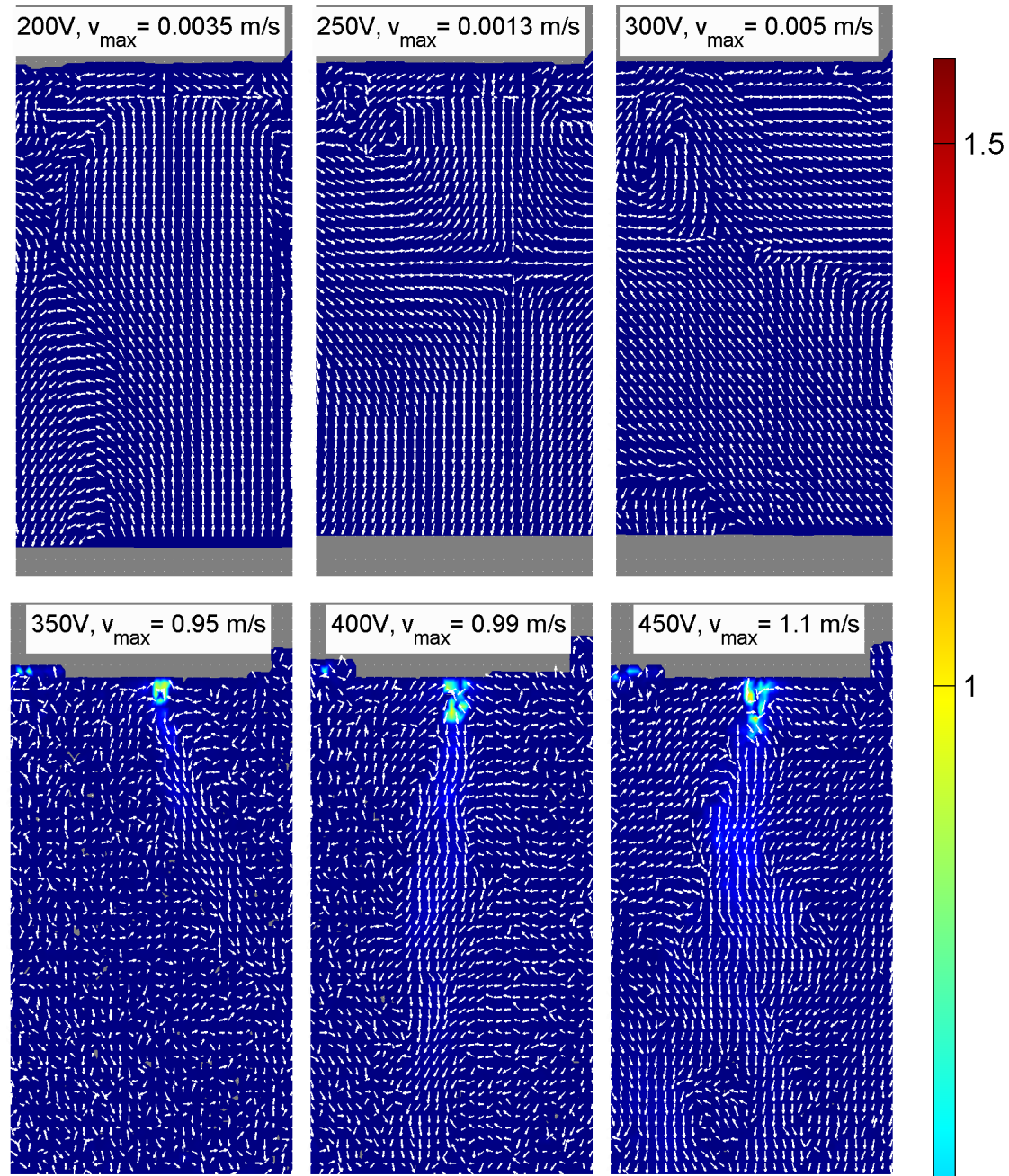

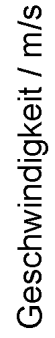
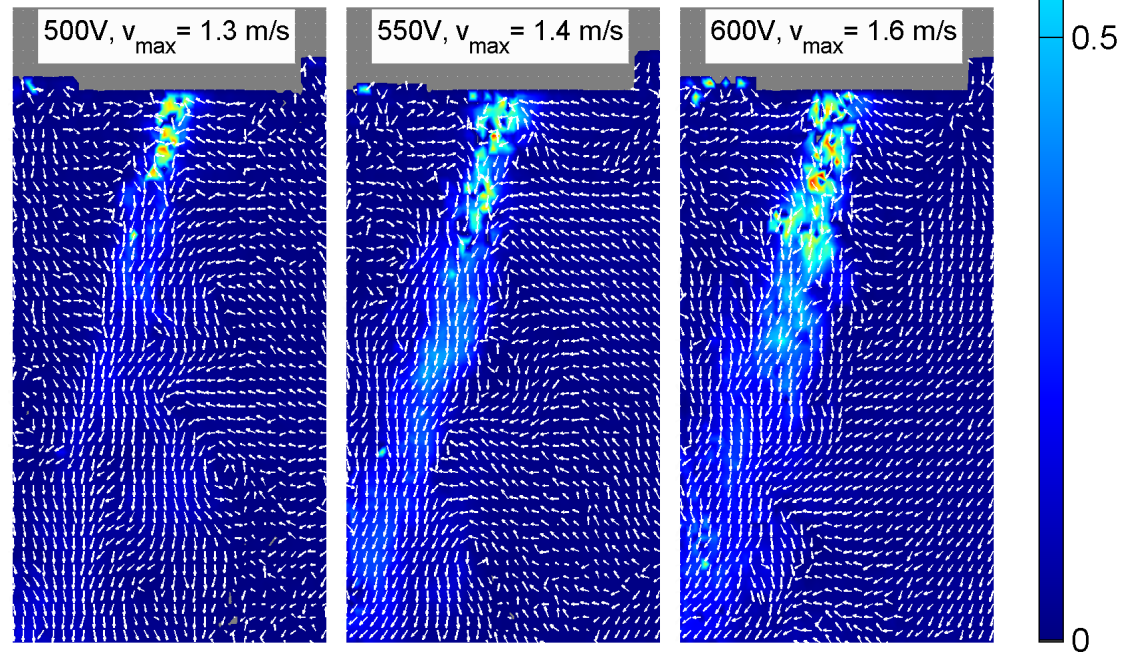

Abbildung 4.22.: Strömungsfeld unter der Sonotrode bei $10^{\circ} \mathrm{C}$ und $50 \%$ Gasgehalt bei Normaldruck. Die Geschwindigkeit wird durch die Farbe, die Richtung der Strömung durch Pfeile dargestellt. Die grauen Bereiche sind Regionen mit fehlenden Messwerten (Maskierung der Sonotrode, schlechte Beleuchtung oder fehlende Partikel). 

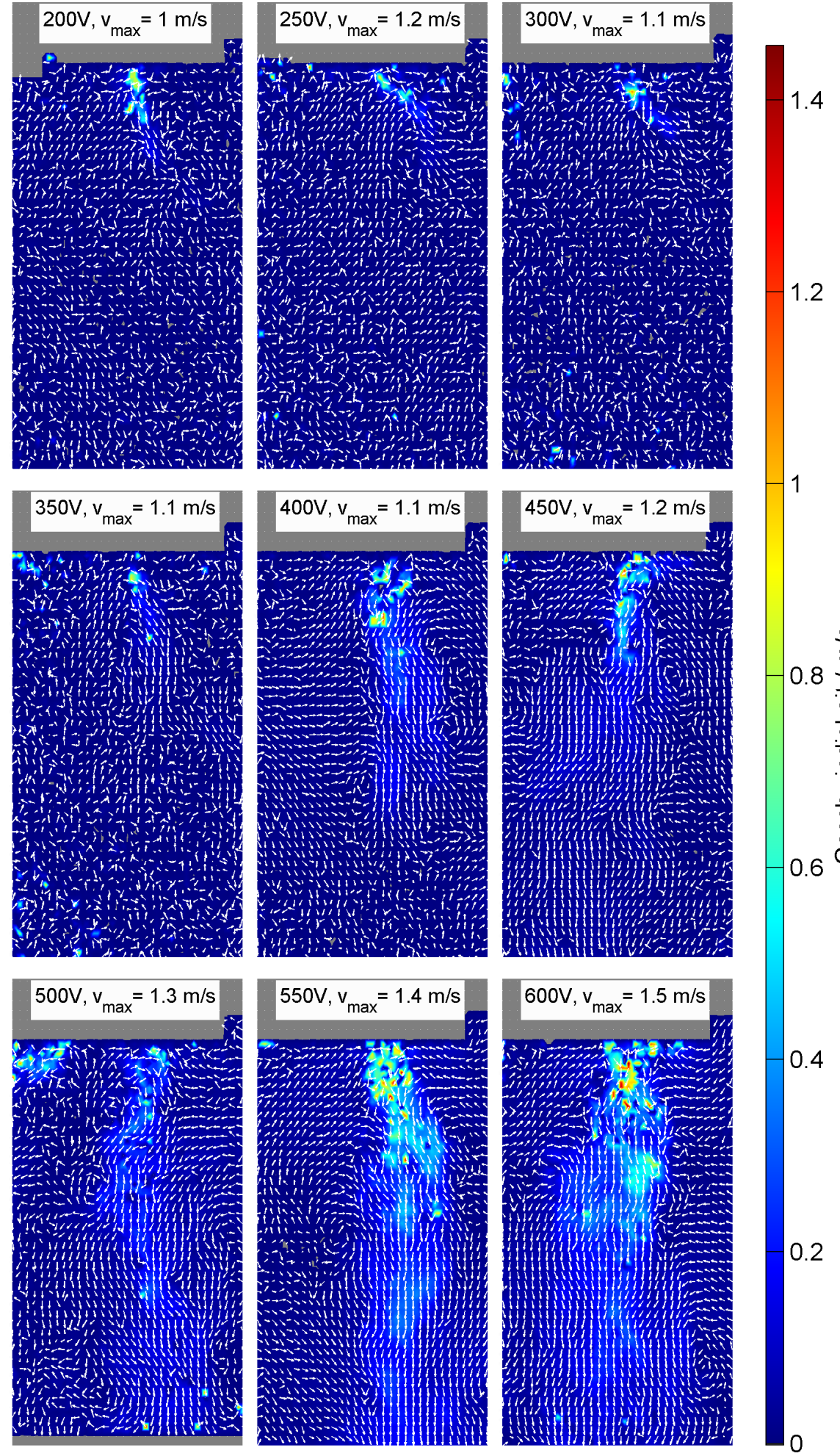

Abbildung 4.23.: Strömungsfeld unter der Sonotrode bei $10^{\circ} \mathrm{C}$ und $90 \%$ Gasgehalt bei Normaldruck. Die Geschwindigkeit wird durch die Farbe, die Richtung der Strömung durch Pfeile dargestellt. Die grauen Bereiche sind Regionen mit fehlenden Messwerten (Maskierung der Sonotrode, schlechte Beleuchtung oder fehlende Partikel). 

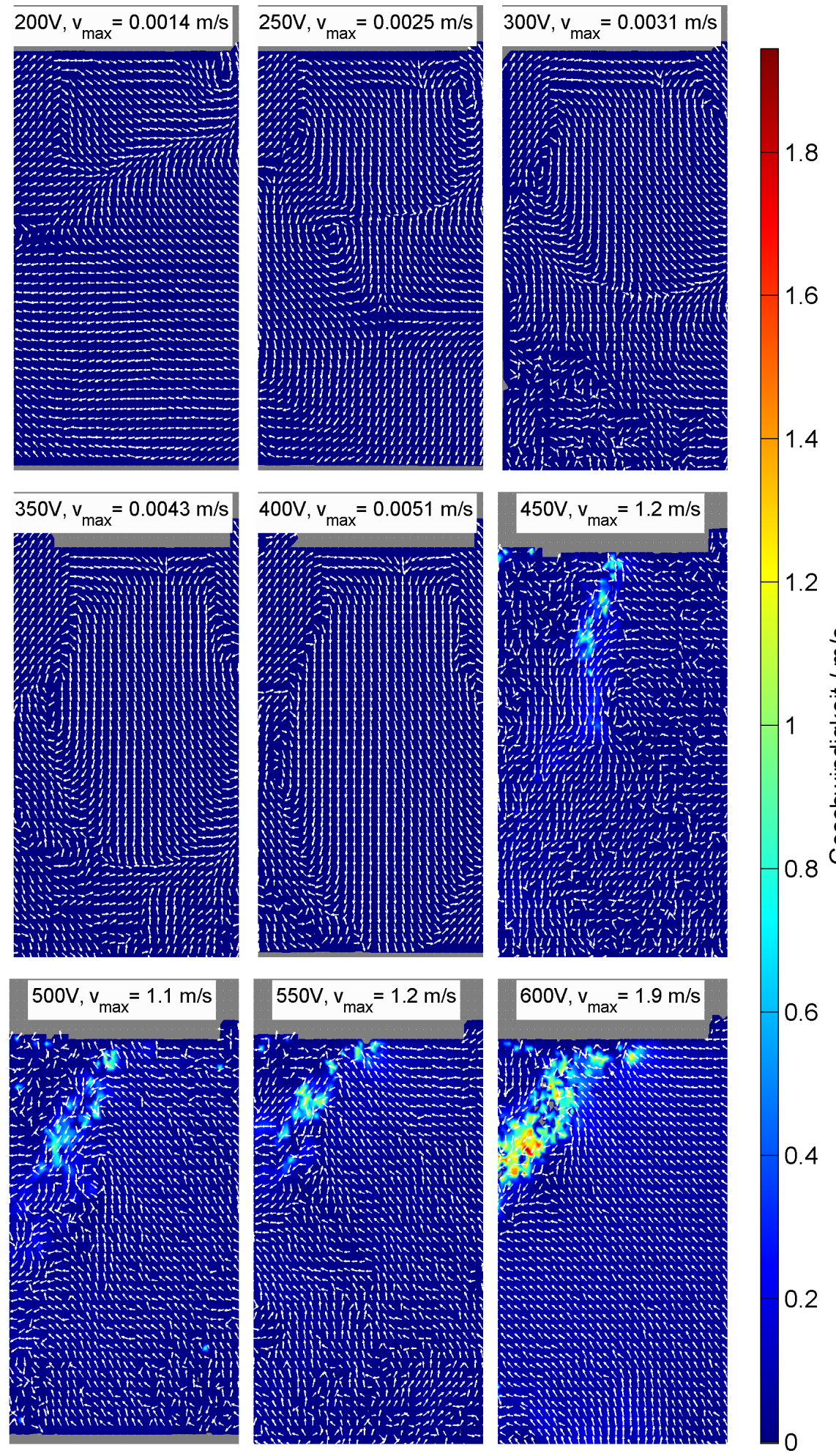

Abbildung 4.24.: Strömungsfeld unter der Sonotrode bei $20^{\circ} \mathrm{C}$ und $5 \%$ Gasgehalt bei Normaldruck. Die Geschwindigkeit wird durch die Farbe, die Richtung der Strömung durch Pfeile dargestellt. Die grauen Bereiche sind Regionen mit fehlenden Messwerten (Maskierung der Sonotrode, schlechte Beleuchtung oder fehlende Partikel). 
Hauptströmungsfeldes mit zunehmender Spannung deutlich stärker gerichtet. Die Kavitation bei $20^{\circ} \mathrm{C}$ und $50 \%$ Gasgehalt setzt wie bei $10^{\circ} \mathrm{C}$ und $90 \%$ Gasgehalt bereits unterhalb von $200 \mathrm{~V}$ ein (siehe Abbildung 4.25). Dies ist hier hauptsächlich an der maximalen Strömungsgeschwindigkeit zu erkennen. Bei $200 \mathrm{~V}$ ist nur direkt an der Sonotrode ein sehr kleiner Bereich mit erhöhter Strömungsgeschwindigkeit zu sehen. Das gesamte Strömungsfeld ist bei niedrigen Spannungen verrauscht und wird mit zunehmender Spannung, maximaler Strömungsgeschwindigkeit und Größe des angetriebenen Strömungsfeldes deutlich gerichteter. Bei $600 \mathrm{~V}$ werden hier bis zu $1.9 \mathrm{~m} \mathrm{~s}^{-1}$ erreicht und fast das ganze Beobachtungsfenster wird vom Hauptströmungsfeld ausgefüllt. Die Aufnahmen bei $20^{\circ} \mathrm{C}$ und $90 \%$ Gasgehalt in Abbildung 4.26 zeigen ebenfalls ein Einsetzen der Kavitation unterhalb von 200 V. Hier ist die maximale Strömungsgeschwindigkeit bereits wesentlich höher als bei 50\% Gasgehalt und das Strömungsfeld ist schon deutlicher zu erkennen. Mit zunehmender Spannung nehmen auch die maximalen Strömungsgeschwindigkeiten zu und das sichtbare Feld hoher Strömungsgeschwindigkeiten wird größer. Bei $600 \mathrm{~V}$ werden bis zu $1.8 \mathrm{~m} \mathrm{~s}^{-1}$ erreicht. Außerhalb des Hauptströmungsfeldes sind Verwirbelungen zu erkennen.

\section{Strömungen bei $30^{\circ} \mathrm{C}$}

Durch den etwas kleineren Bildausschnitt ist das Strömungsfeld bei $30{ }^{\circ} \mathrm{C}$ und $5 \%$ Gasgehalt bereits bei $350 \mathrm{~V}$ fast bildfüllend ausgeprägt (Abbildung 4.27). Die Maximalgeschwindigkeit nimmt von 350 bis $450 \mathrm{~V}$ monoton zu. Erst bei $550 \mathrm{~V}$ setzt Kavitation ein. Die Hauptströmungsrichtung zeigt nach links und die Maximalgeschwindigkeiten sind mit 1.9 und $2.1 \mathrm{~m} \mathrm{~s}^{-1}$ recht hoch. Bei $30^{\circ} \mathrm{C}$ und $50 \%$ Gasgehalt in Abbildung 4.28 ist bereits bei $350 \mathrm{~V}$ die Kavitationsschwelle überschritten. Es ist ein kleiner, schnell strömender Bereich direkt an der Sonotrode und ein relativ schmaler strömender Jet von der Sonotrode weg zu erkennen. Mit zunehmender Spannung wird das Strömungsfeld größer und wechselt je nach Spannung die Richtung. Die Maximalgeschwindigkeit ist größtenteils identisch, nimmt im Mittel aber mit zunehmender Spannung zu und liegt bei $600 \mathrm{~V}$ bei $2 \mathrm{~m} \mathrm{~s}^{-1}$. Neben dem Hauptströmungsfeld sind wieder Verwirbelungen zu erkennen. Das Strömungsfeld bei $30^{\circ} \mathrm{C}$ ist in Abbildung 4.29 dargestellt. Bereits bei $200 \mathrm{~V}$ ist die Kavitationsschwelle überschritten. Direkt an der Sonotrode ist ein kleiner schnell strömender Bereich mit einem von der Sonotrode weg nach rechts gerichteten Jet zu erkennen. Dieser Jet wird mit zunehmender Spannung immer größer und die maximal vorkommende Geschwindigkeit nimmt bis zu $1.8 \mathrm{~m} \mathrm{~s}^{-1} \mathrm{zu}$. Außerhalb des Jets ist die Strömung bei niedrigen Spannungen noch verrauscht und wird mit Zunahme der Spannung immer besser aufgelöst und glatter. Außerdem sind außerhalb des Jets am Rand des Strömungsfeldes Wirbel zu erkennen. 

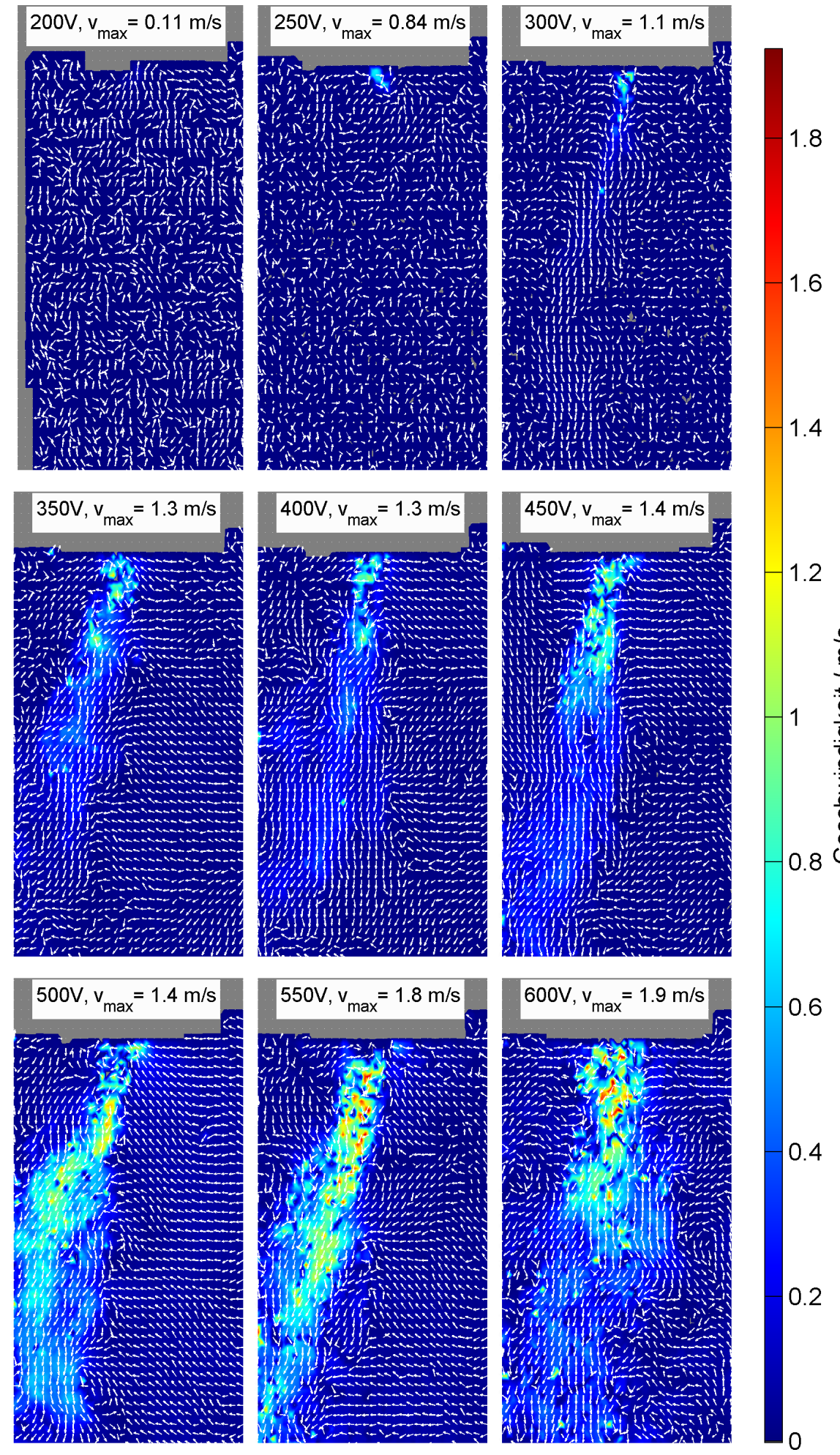

Abbildung 4.25.: Strömungsfeld unter der Sonotrode bei $20^{\circ} \mathrm{C}$ und $50 \%$ Gasgehalt bei Normaldruck. Die Geschwindigkeit wird durch die Farbe, die Richtung der Strömung durch Pfeile dargestellt. Die grauen Bereiche sind Regionen mit fehlenden Messwerten (Maskierung der Sonotrode, schlechte Beleuchtung oder fehlende Partikel). 

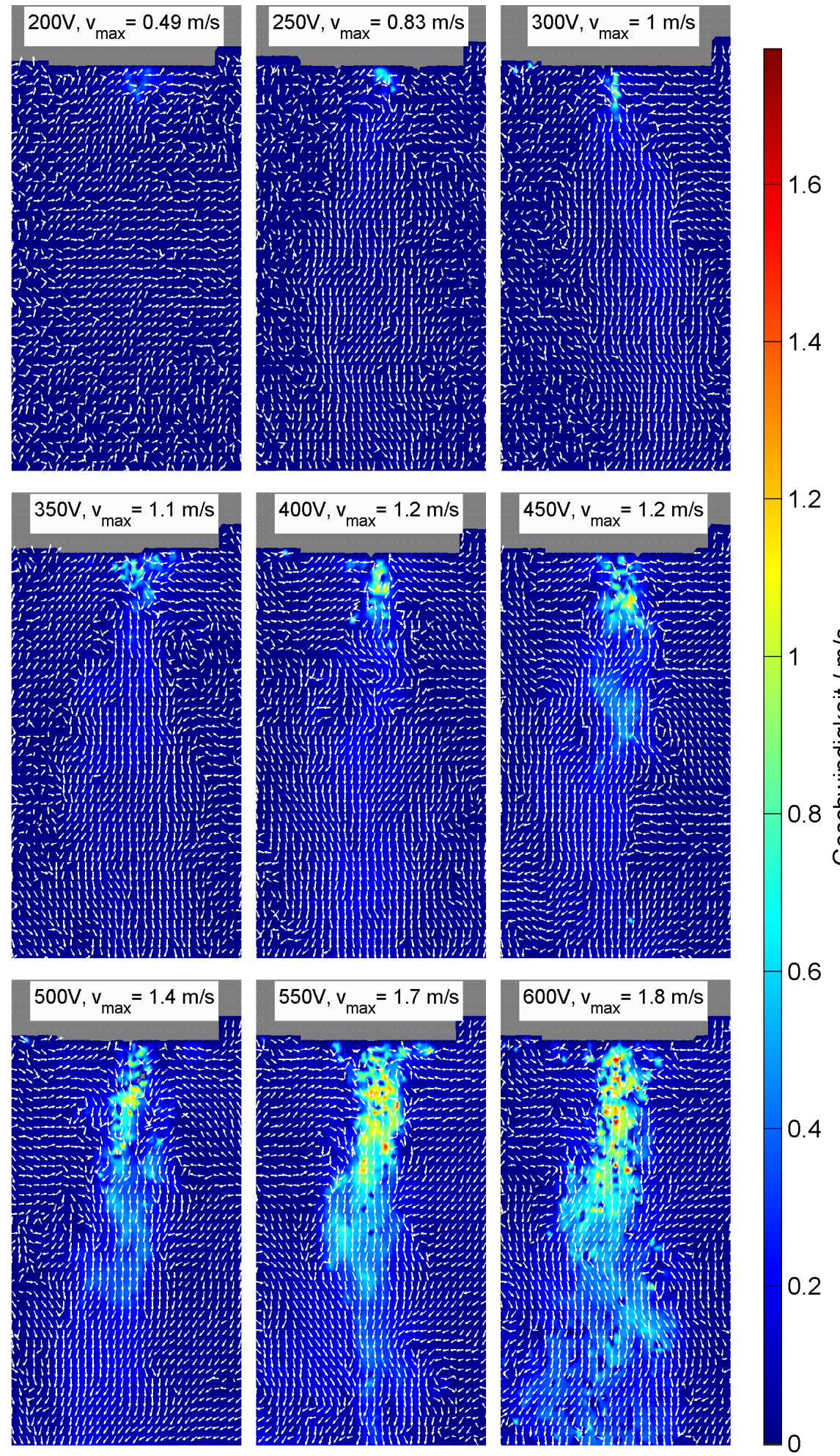

Abbildung 4.26.: Strömungsfeld unter der Sonotrode bei $20^{\circ} \mathrm{C}$ und $90 \%$ Gasgehalt bei Normaldruck. Die Geschwindigkeit wird durch die Farbe, die Richtung der Strömung durch Pfeile dargestellt. Die grauen Bereiche sind Regionen mit fehlenden Messwerten (Maskierung der Sonotrode, schlechte Beleuchtung oder fehlende Partikel). 

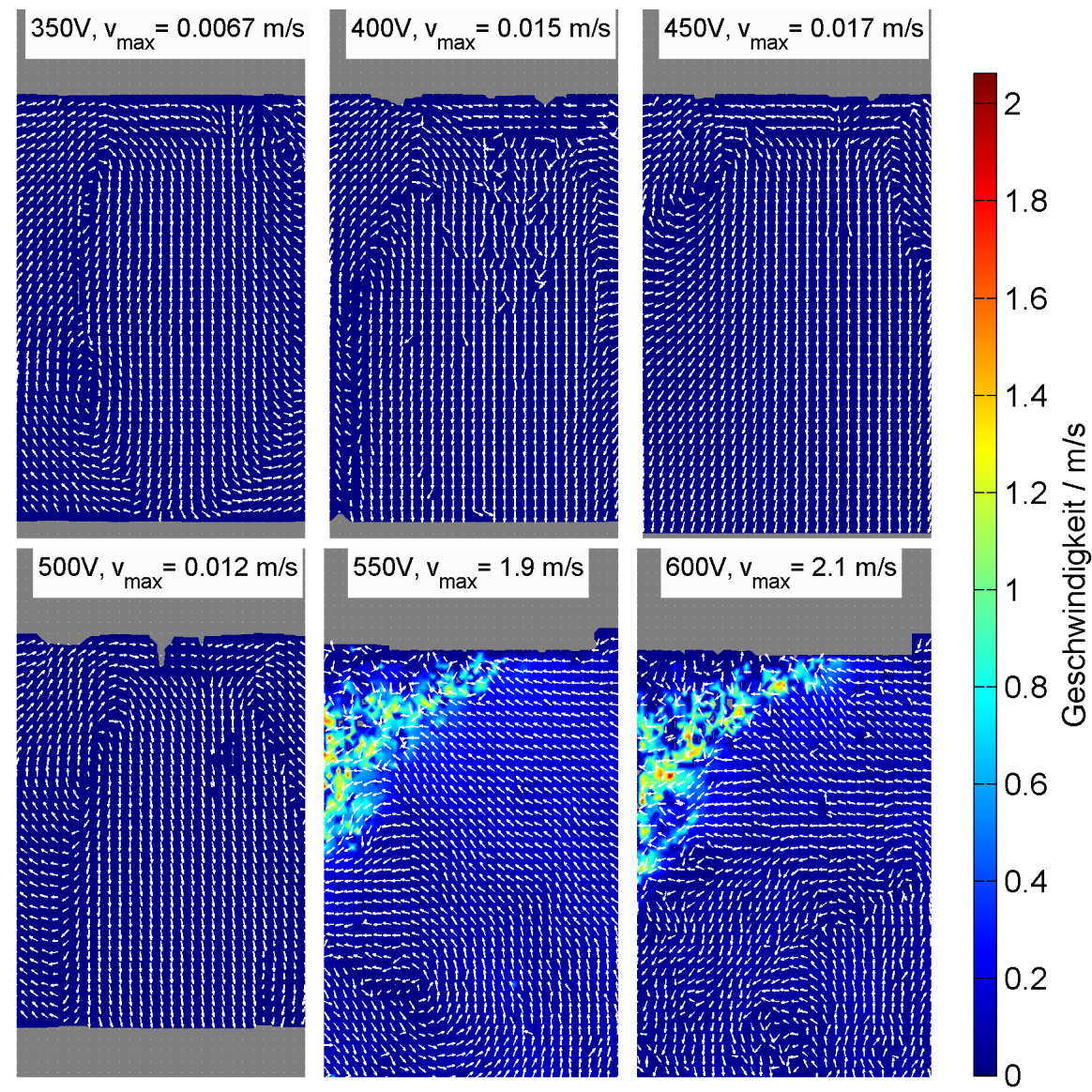

Abbildung 4.27.: Strömungsfeld unter der Sonotrode bei $30^{\circ} \mathrm{C}$ und $5 \%$ Gasgehalt bei Normaldruck. Die Geschwindigkeit wird durch die Farbe, die Richtung der Strömung durch Pfeile dargestellt. Die grauen Bereiche sind Regionen mit fehlenden Messwerten (Maskierung der Sonotrode, schlechte Beleuchtung oder fehlende Partikel).

\subsection{Zusammenhang zwischen Kavitations- und Strömungsfeld}

In diesem Kapitel soll anhand von ausgewählten Beispielen auf den Zusammenhang zwischen Kavitations- und Strömungsfeld eingegangen werden. Dazu wurden einzelne Sequenzen ausgewählt, und es wurde das Geschwindigkeitsfeld in Form von geschwindigkeitsproportionalen Vektoren über die Aufnahme der Kavitationsblasen gelegt.

Es ist zu erkennen, dass die Zonen hoher Geschwindigkeit im oberen Bereich in den Teilabbildungen in Abbildung 4.30 mit dem Kavitationsblasenfeld korrelieren. Im unteren Bereich des Ausschnittes sind teilweise Zonen hoher Geschwindig- 

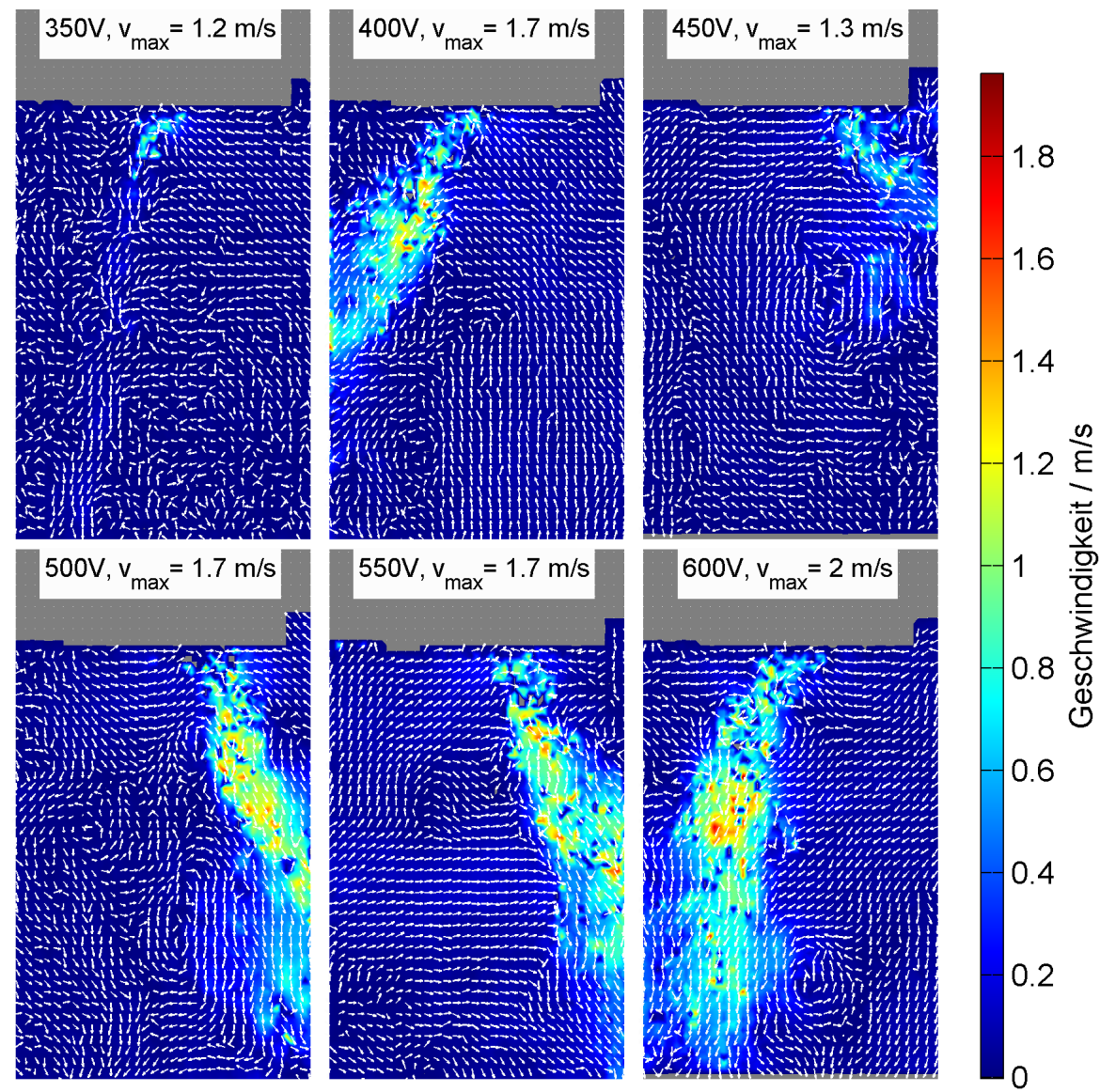

Abbildung 4.28.: Strömungsfeld unter der Sonotrode bei $30^{\circ} \mathrm{C}$ und $50 \%$ Gasgehalt bei Normaldruck. Die Geschwindigkeit wird durch die Farbe, die Richtung der Strömung durch Pfeile dargestellt. Die grauen Bereiche sind Regionen mit fehlenden Messwerten (Maskierung der Sonotrode, schlechte Beleuchtung oder fehlende Partikel).

keit zu erkennen, obwohl keine Blasen sichtbar sind. Allerdings sind kleine Blasen unter der Aufösungsgrenze nicht sichtbar. Innerhalb des Blasenfeldes sind zum Teil auch Zonen niedriger Geschwindigkeit zu erkennen. Dies liegt zum einen an einer Abschattung der Fluoreszenzpartikel durch Kavitationsblasen, zum anderen an einer starken räumlichen Schwankung der Blasenwolke, die sich auch leicht vor oder hinter der Lichtschnittebene befinden kann. In Abbildung 4.31 ist die Ausbreitung einer Blasenwolke unter der Sonotrode abgebildet. Die Bilder wurden in möglichst konstantem Abstand so ausgewählt, dass die Blasen maximal aufgeschwungen sind. Die Bewegung der Blasenwolke weg von der Sonotrode, die sich oben außerhalb des Bildes befindet, ist gut zu erkennen. Dabei lässt sich ausmachen, dass die Regionen hoher Blasendichte stark mit den Regionen hoher 

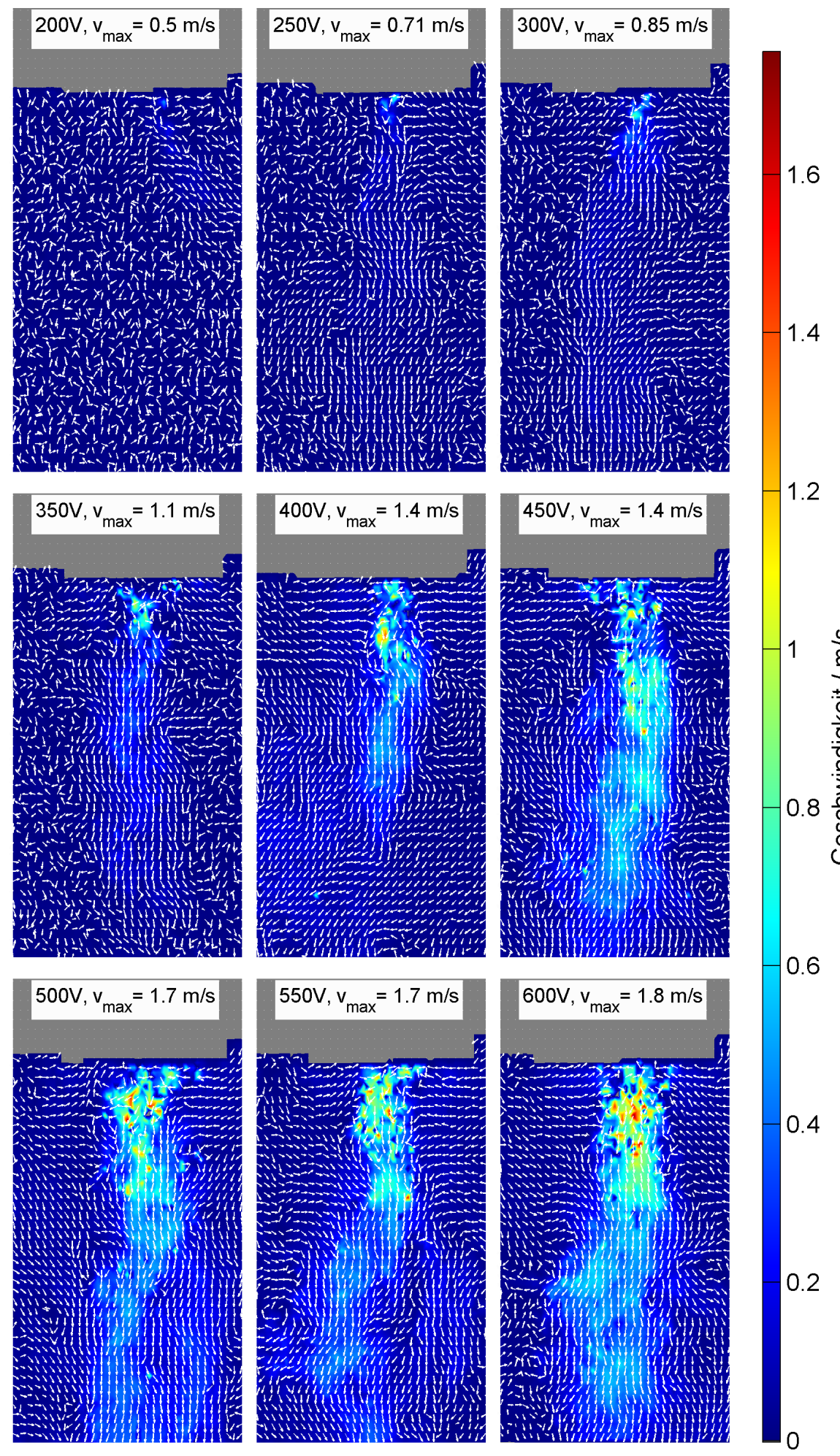

Abbildung 4.29.: Strömungsfeld unter der Sonotrode bei $30^{\circ} \mathrm{C}$ und $90 \%$ Gasgehalt bei Normaldruck. Die Geschwindigkeit wird durch die Farbe, die Richtung der Strömung durch Pfeile dargestellt. Die grauen Bereiche sind Regionen mit fehlenden Messwerten (Maskierung der Sonotrode, schlechte Beleuchtung oder fehlende Partikel). 


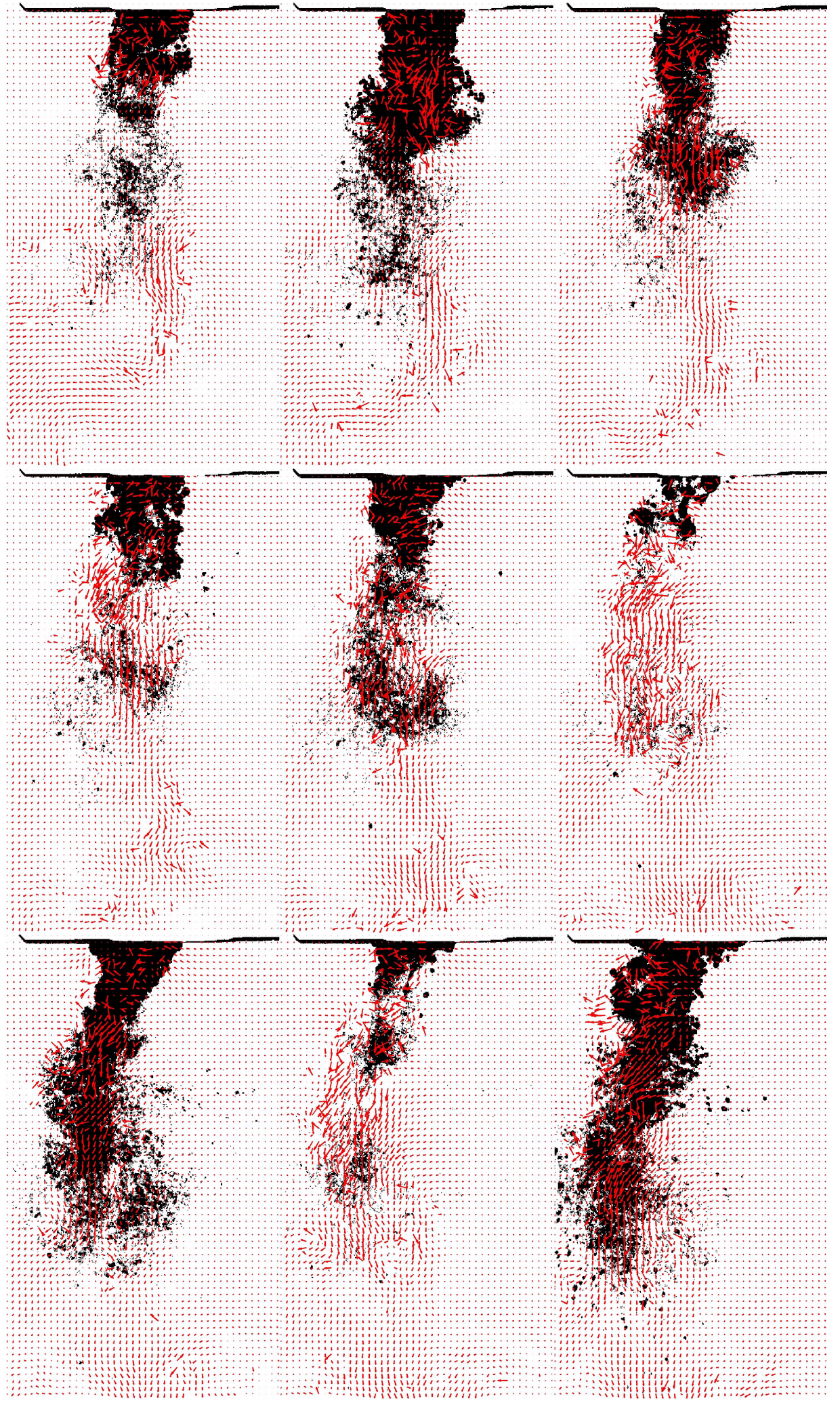

Abbildung 4.30.: Blasen- und Strömungsfeld unter der Sonotrode bei $30^{\circ} \mathrm{C}$ und $50 \%$ Gasgehalt. Belichtungszeiten 1/6000 s für das Geschwindigkeitsfeld und 1.5 us für das Kavitationsblasenfeld. Aufnahmerate 5000 Bps, Zeit zwischen den einzelnen Abbildungen $2 \mathrm{~ms}$. 
Geschwindigkeit übereinstimmen. Außerdem weisen die Geschwindigkeitsvektoren innerhalb der Blasenwolke eine wesentlich größere Unordnung auf. Dieser Effekt scheint mit der Blasendichte zu korrelieren. Bei geringerer Blasendichte sind die Geschwindigkeitsvektoren stärker gleichförmig in eine Vorzugsrichtung ausgerichtet. Des Weiteren nimmt die Breite der Blasenwolke mit der Entfernung von der Sonotrode zu.
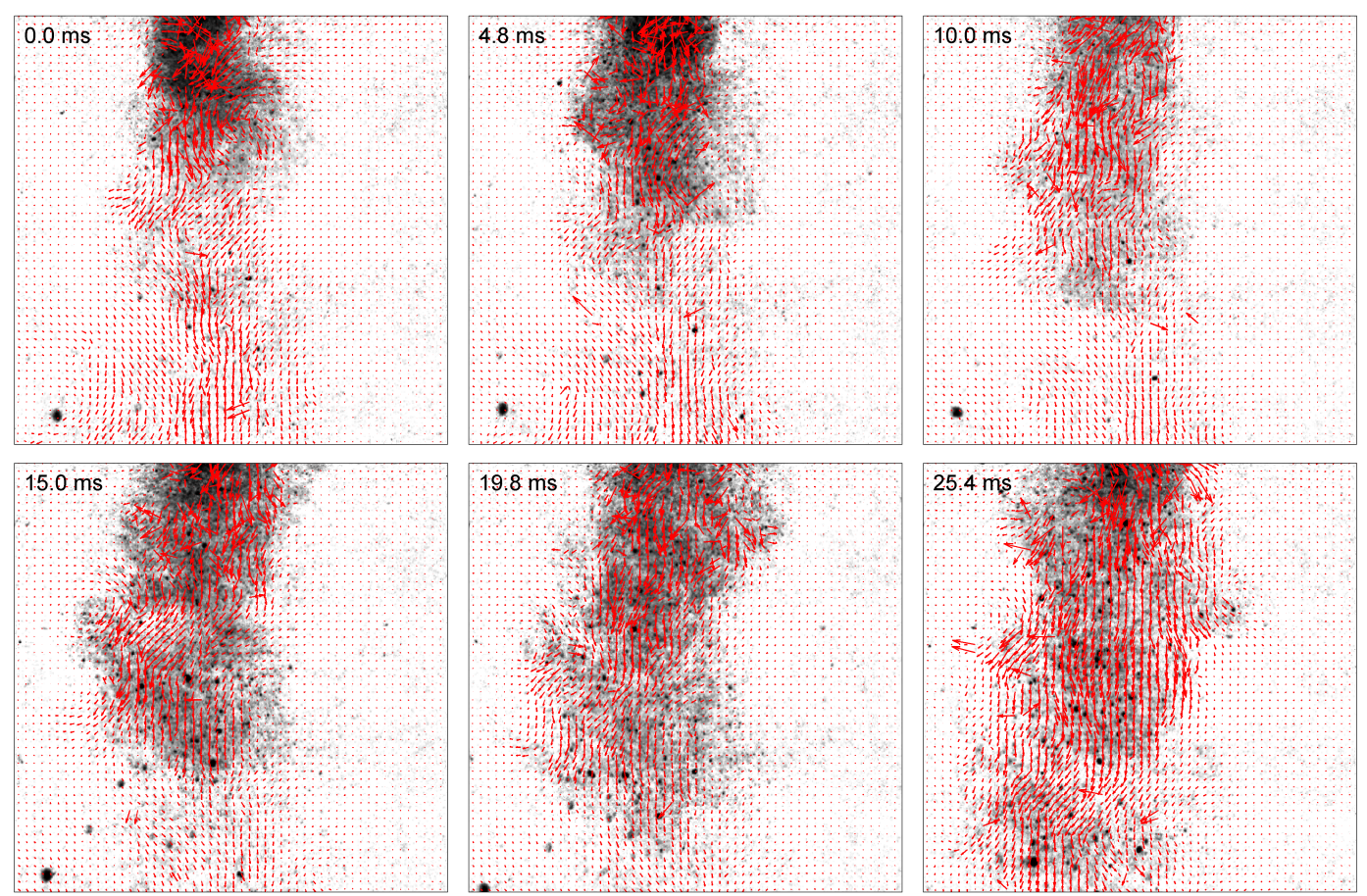

Abbildung 4.31.: Ausschnitt des Blasen- und Strömungsfeldes unter der Sonotrode bei $20^{\circ} \mathrm{C}$ und $90 \%$ Gasgehalt. Belichtungszeiten $1 / 6000$ s für das Geschwindigkeitsfeld und 1.5 s für das Kavitationsblasenfeld. Aufnahmerate $5000 \mathrm{Bps}$, die Zeit zwischen den einzelnen Abbildungen wurde so gewählt, dass die Blasen immer maximal aufgeschwungen sind. Grafik entnommen aus [93] 


\subsection{Energieabsorption in Abhängigkeit von Tem- peratur und Gasgehalt}

Um die absorbierte Energie mit und ohne Kavitation bei gleichem Schalldruck vergleichen zu können, wurde die kinetische Energiedichte in der Strömung berechnet und für die verschiedenen Temperaturen und Gasgehalte aufgetragen. Hierzu wurde an jedem Messpunkt des geglätteten (Medianfilterung über eine $2 \times 2$-Matrix an jedem Punkt) Strömungsfeldes der Betrag des Geschwindigkeitsvektors gebildet und daraus die kinetische Energiedichte $E_{k i n}=1 / 2 \rho u_{2}^{2}$ errechnet. Diese wurde anschließend erst in jedem Bild und dann über die Dauer des gesamten Films gemittelt. Die Ergebnisse sind in den folgenden Kapiteln jeweils in linearer und in logarithmischer Auftragung aufgeführt. Die eingetragenen Fehlerbalken bezeichnen in diesem Fall die Standardabweichung der mittleren kinetischen Energiedichte über die Zeit. Ein großer Fehler zeigt also eine große Schwankung der Energiedichte über die Zeit an, wenn die Kavitation zum Beispiel kurzzeitig aussetzt. Ein fehlender Balken nach unten in der logarithmischen Auftragung bedeutet einen höheren Fehler- als Messwert, weshalb der Fehlerbalken ins Negative gehen würde. Die gefitteten Kurven sind Gleichungen der Form $y=a U_{\mathrm{el}}^{4}$. Die in den logarithmischen Darstellungen eingezeichnete gestrichelte Gerade zeigt die Grenze zwischen der Energiedichte ohne und der Energiedichte mit Kavitation an. Bei Wahl eines anderen Beobachtungsfensters (Bildausschnitt anderer Größe oder Position im Vergleich zum Strömungsfeld) verschiebt sich diese Gerade zu höheren oder niedrigeren Energien. Wenn für einen Parametersatz sowohl Messungen mit als auch ohne Kavitation vorlagen, wurden beide Energiedichten berechnet und in den Grafiken eingetragen.

\section{Untersuchungen bei $10^{\circ} \mathrm{C}$}

In Abbildung 4.32 ist die kinetische Energiedichte über der an der Sonotrode anliegenden Spannung linear (Abbildung 4.32(a)) und logarithmisch (Abbildung $4.32(\mathrm{~b})$ ) bei $10^{\circ} \mathrm{C}$ aufgetragen. Es ist ein deutlicher Anstieg der Energiedichte mit der anliegenden Spannung zu erkennen. Die Werte für 5\% Gasgehalt streuen stark und weisen speziell bei den höheren Spannungen hohe Fehler auf. Die Messungen bei 50\% Gasgehalt zeigen einen ruhigeren Verlauf und die höchste Energiedichte. Bei 90\% Gasgehalt liegen die Werte etwas unter den Werten bei $50 \%$ Gasgehalt. Von $450 \mathrm{~V}$ zu $500 \mathrm{~V}$ ist ein Sprung der Werte nach unten zu erkennen. Dies könnte an einer leichten Verschiebung der Lichtschnittebene liegen, so dass die Strömung nicht mehr mittig, sondern am Rand aufgenommen wird. Der tatsächliche Verlauf der Messwerte folgt in diesem Fall eher $U_{\mathrm{el}}^{6}$. Aufnahmen 


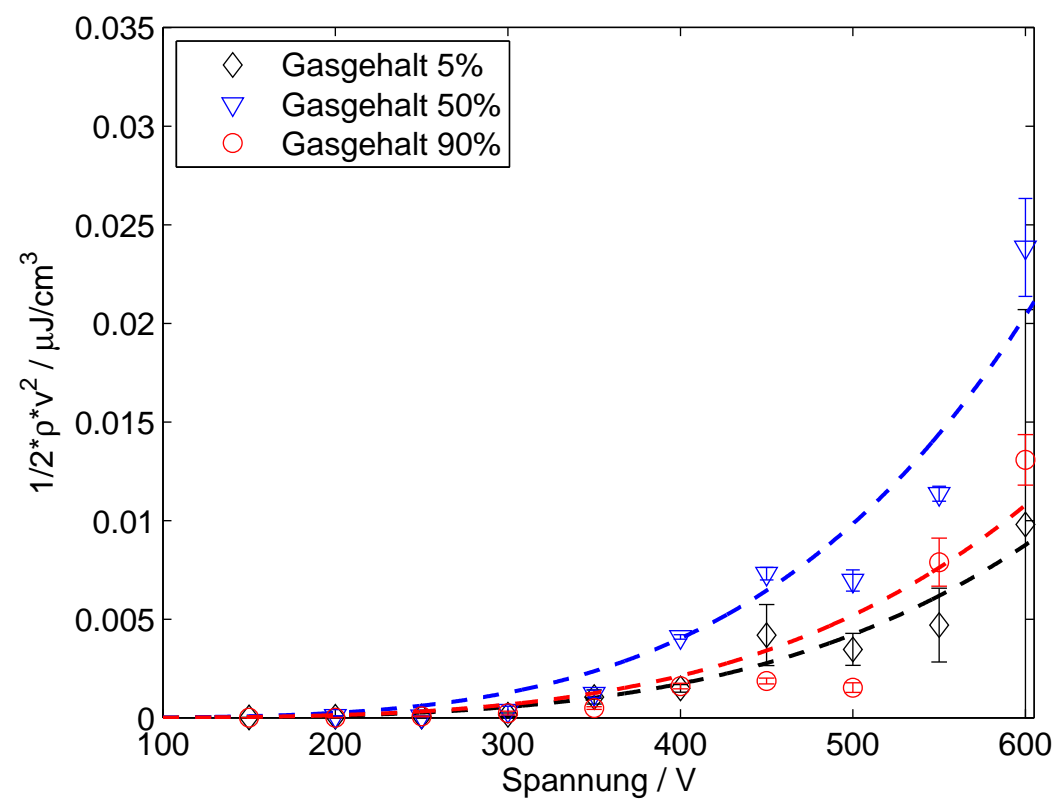

(a) lineare Auftragung

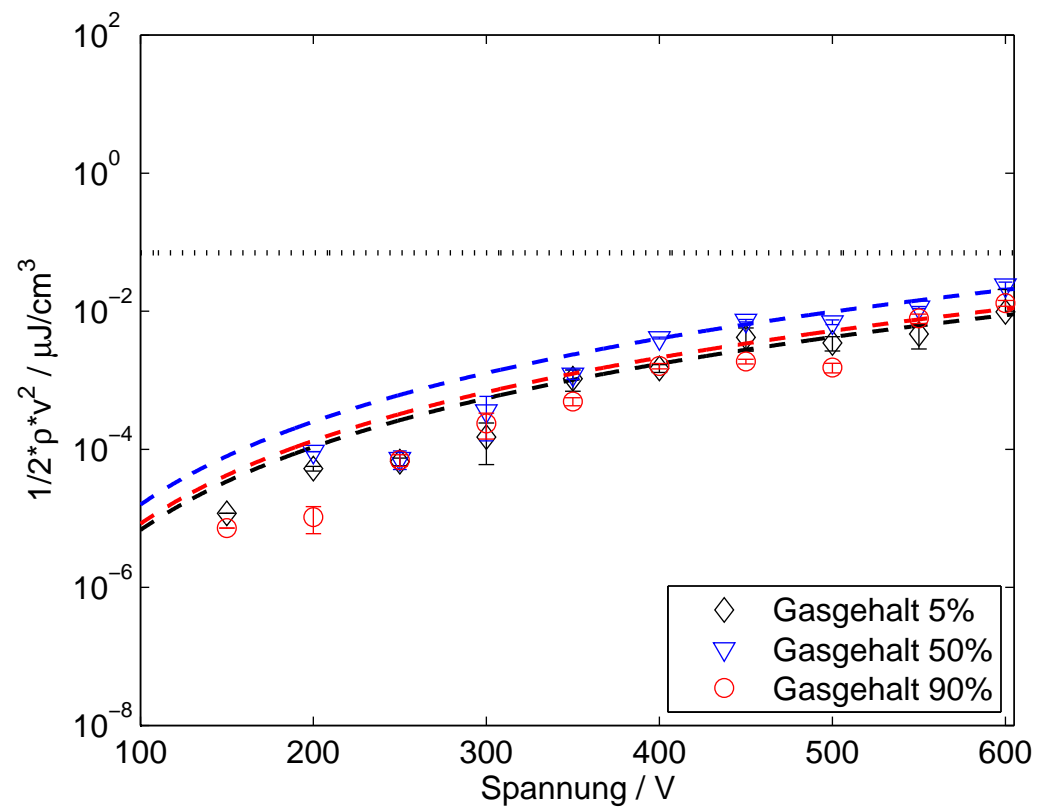

(b) logarithmische Auftragung

Abbildung 4.32.: Kinetische Energiedichte aufgetragen über der an der Sonotrode anliegenden Spannung. Der Sauerstoffgehalt betrug $6 \pm 1$, $52 \pm 3$ und $95 \pm 3$ Prozent und die Temperatur $10 \pm 2{ }^{\circ} \mathrm{C}$. Durch Anlegen von 2 bar Überdruck wurde die Kavitation unterdrückt. Die angepassten Kurven verlaufen proportional zu $U_{\mathrm{el}}^{4}$. 


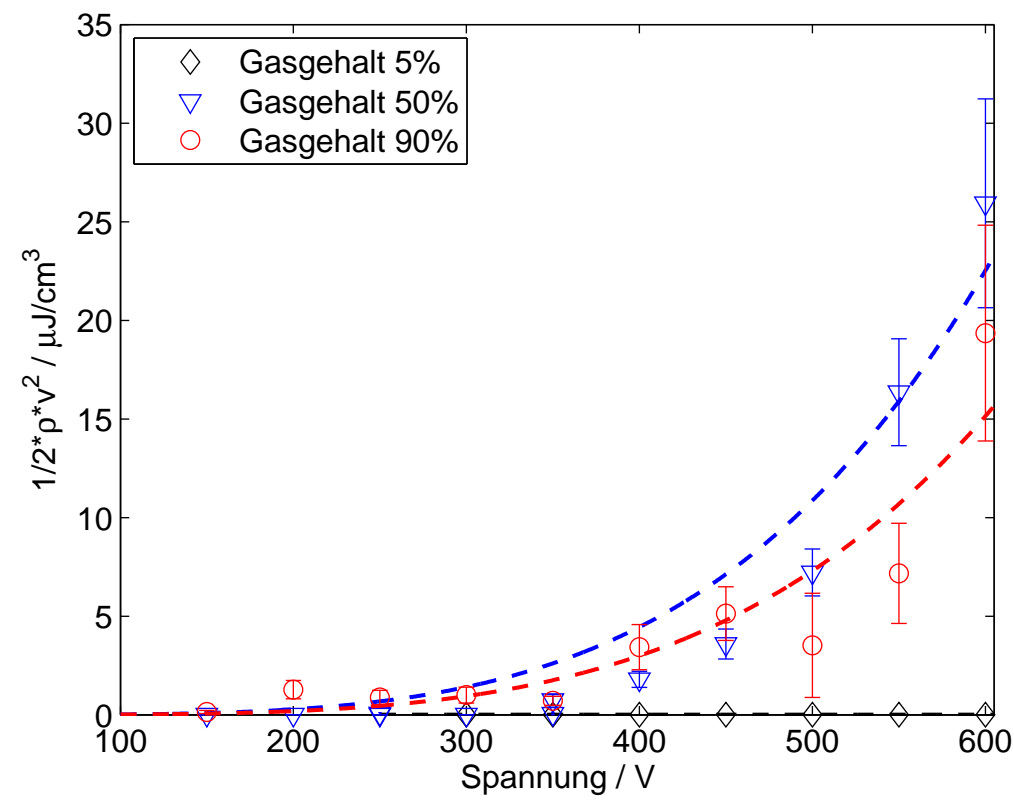

(a) lineare Auftragung

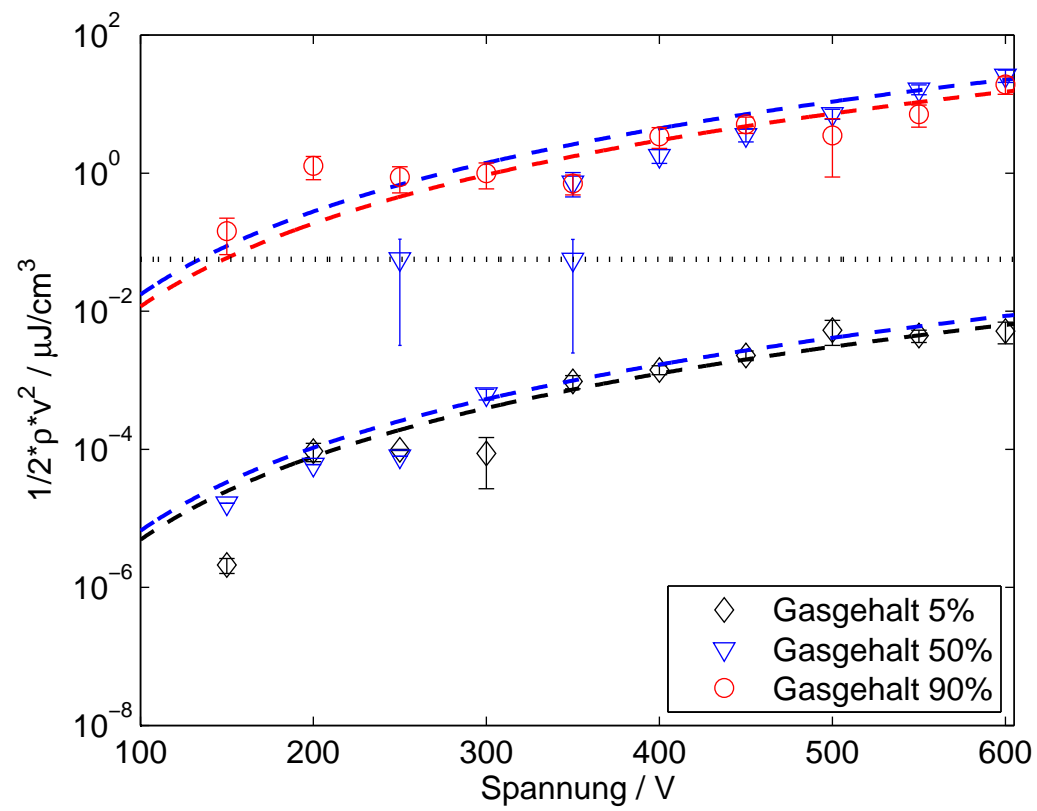

(b) logarithmische Auftragung

Abbildung 4.33.: Kinetische Energiedichte aufgetragen über der an der Sonotrode anliegenden Spannung. Der Sauerstoffgehalt betrug $6 \pm 1$, $52 \pm 3$ und $95 \pm 3$ Prozent und die Temperatur $10 \pm 2{ }^{\circ} \mathrm{C}$. Die angenäherten Kurven sind proportional zu $U_{\mathrm{el}}^{4}$. 
bei $10^{\circ} \mathrm{C}$ und Normaldruck zeigen wieder einen klaren Anstieg der kinetischen Energiedichte zu höheren Spannungen. Auch hier verlaufen die Kurven bei 5 und $50 \%$ Gasgehalt relativ glatt. Die Messwerte bei $90 \%$ Gasgehalt zeigen eine größere Streuung. In Abbildung 4.33(b) ist das Überschreiten der Kavitationsschwelle bei $50 \%$ Gasgehalt besonders gut zu erkennen. So zeigen die Messungen bei $5 \%$ Gasgehalt durchgehend niedrige Werte und einen Verlauf proportional zu $U_{\mathrm{el}}^{4}$. Die Messwerte bei $50 \%$ Gasgehalt hingegen weisen von $350 \mathrm{zu} 400 \mathrm{~V}$ einen deutlichen Sprung auf. Hier wechselt die Steigung des tatsächlichen Verlaufs von $U_{\mathrm{el}}^{4} \mathrm{zu} U_{\mathrm{el}}^{8}$. Die Messwerte bei 90\% Gasgehalt liegen sofort bei höheren Energiedichten. Die Kavitationsschwelle ist in diesem Fall bereits unter $150 \mathrm{~V}$ überschritten.

\section{Untersuchungen bei $20^{\circ} \mathrm{C}$}

In Abbildung 4.34 sind die Werte für $20^{\circ} \mathrm{C}$ aufgetragen. Die Werte bei $5 \%$ Gasgehalt streuen stark und liegen zu großen Teilen nicht auf der angepassten Kurve. Bei 50 und 90\% Gasgehalt passen die Kurven recht gut. Die Maximalwerte liegen hier deutlich höher als bei $10^{\circ} \mathrm{C}$. Dies hängt mit einem Einsetzen der Kavitation bei $50 \%$ Gasgehalt und $450 \mathrm{~V}$ zusammen. Messwerte ohne Kavitation sind hier leider nicht vorhanden. In der logarithmischen Auftragung (Abbildung 4.34(b)) ist ein deutlicher Sprung der kinetischen Energiedichte von $450 \mathrm{zu} 500 \mathrm{~V}$ zu erkennen. Dass der Sprung nicht bereits bei $450 \mathrm{~V}$ erfolgt, liegt an der Wahl des Auswertungsfensters. Dadurch wird das durch eine einzelne Blase an der Sonotrodenspitze erzeugte sehr kleine Strömungsfeld zu großen Teilen ausgeblendet. Auch hier liegen unterhalb der Schwelle die Werte für 50\% Gasgehalt wieder am höchsten. Zudem folgen die Messwerte ebenfalls einem Verlauf proportional $\mathrm{zu} U_{\mathrm{el}}^{6}$. Mit Einsetzen der Kavitation bei $50 \%$ Gasgehalt folgt ein exponentieller Verlauf. Die angepassten Kurven folgen mit $U_{\mathrm{el}}^{4}$ dem theoretisch erwarteten Verlauf. Die Messungen bei $20^{\circ} \mathrm{C}$ zeigen bei $5 \%$ Gasgehalt niedrige Werte und somit einen Verlauf komplett unterhalb der Kavitationsschwelle. Bei 50\% Gasgehalt sind zwei Sprünge zu erkennen. Einer in der logarithmischen Auftragung (siehe Abbildung 4.35(b)) von $150 \mathrm{zu} 200 \mathrm{~V}$ und einen deutlicher in der linearen Auftragung (Abbildung 4.35(a)). Der erste Sprung zeigt das Überschreiten der Kavitationsschwelle. Der zweite Sprung ist in den dazugehörigen Filmen im Durchlicht als deutliche Vergrößerung der Blasenwolke zu erklären (auch erkennbar im Strömungsfeld in Abbildung 4.25). Die Werte der Messung bei 90\% Gasgehalt zeigen auch hier einen hohen Verlauf über der Kavitationsschwelle. Wie bei den Messungen bei $10^{\circ} \mathrm{C}$ verlaufen die Messwerte oberhalb der Kavitationsschwelle bei $50 \%$ Gasgehalt ebenfalls proportional zu $U_{\mathrm{el}}^{4}$. Allerdings liegen in diesem Fall auch die Messwerte bei $5 \%$ Gasgehalt in der Nähe einer Kurve proportional zu $U_{\mathrm{el}}^{4}$. Bei 


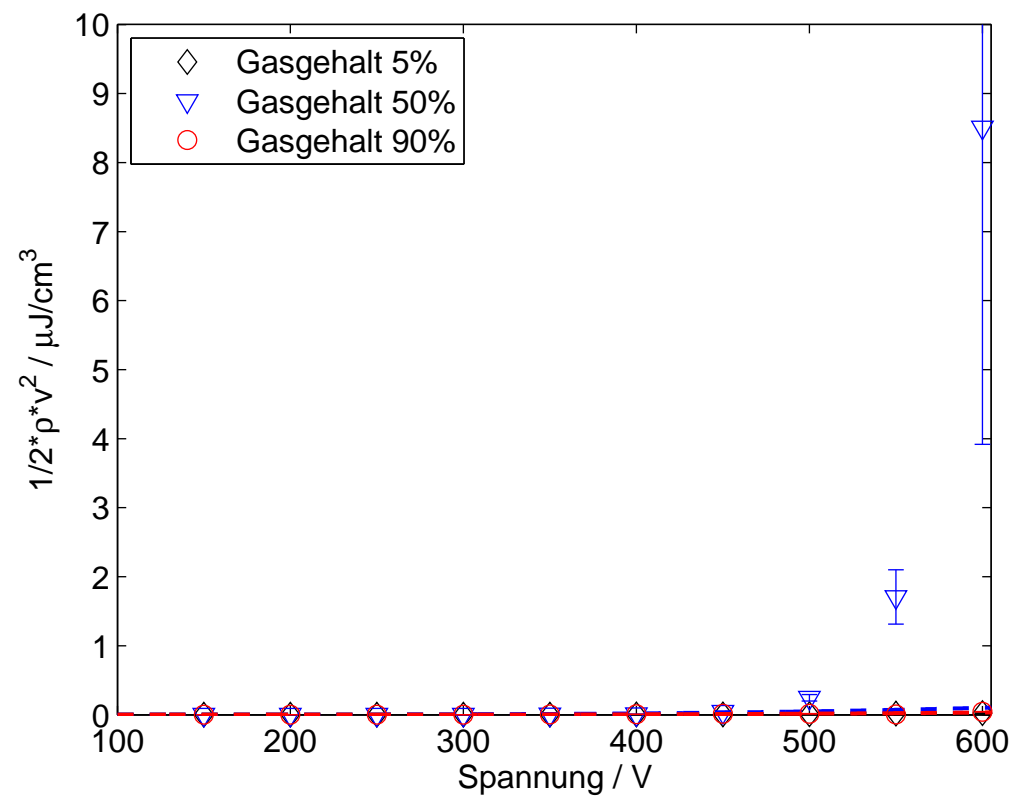

(a) lineare Auftragung

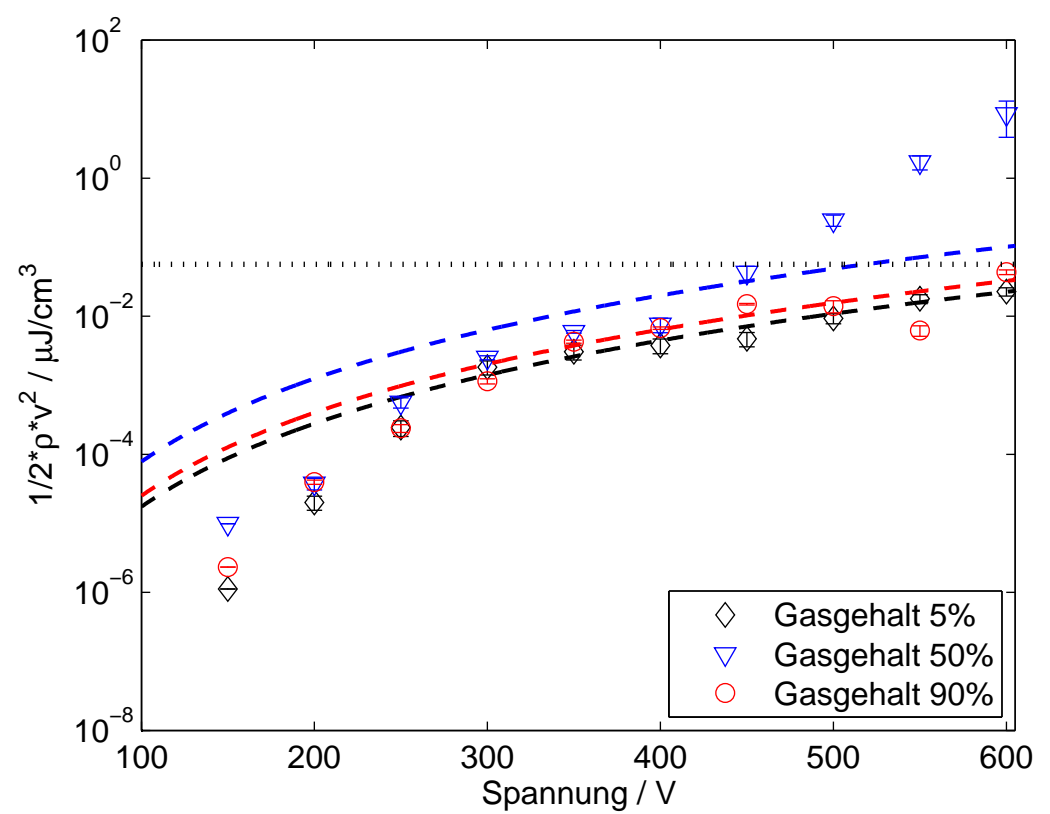

(b) logarithmische Auftragung

Abbildung 4.34.: Kinetische Energiedichte aufgetragen über der an der Sonotrode anliegenden Spannung. Der Sauerstoffgehalt betrug $6 \pm 1$, $51 \pm 2$ und $86 \pm 3$ Prozent und die Temperatur $22 \pm 2{ }^{\circ} \mathrm{C}$. Durch Anlegen von 2 bar Überdruck wurde die Kavitation größtenteils unterdrückt. Die angepassten Kurven verlaufen proportional zu $U_{\mathrm{el}}^{4}$. 


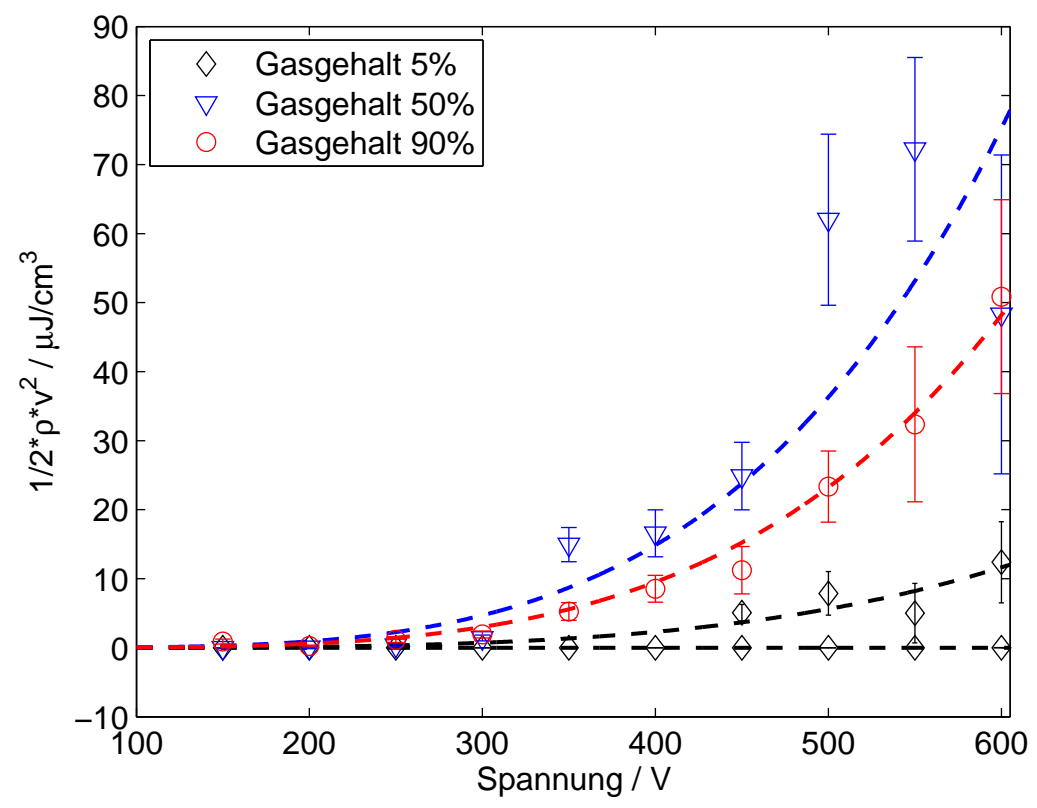

(a) lineare Auftragung

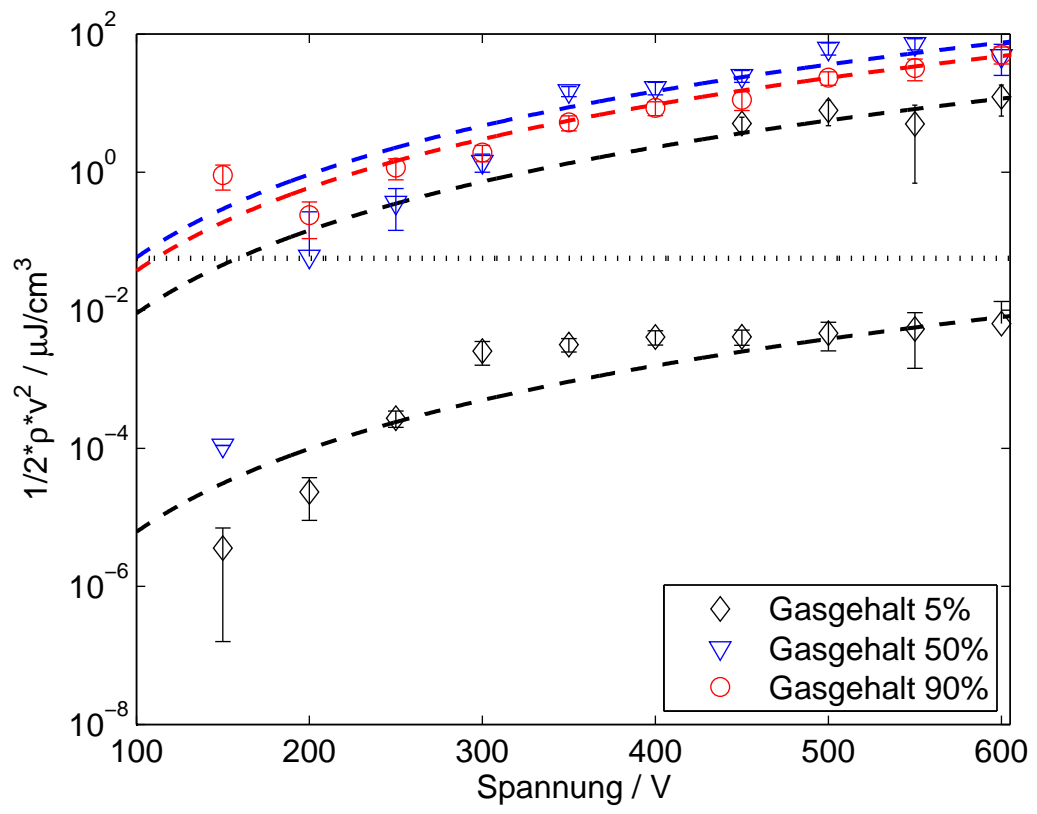

(b) logarithmische Auftragung

Abbildung 4.35.: Kinetische Energiedichte aufgetragen über der an der Sonotrode anliegenden Spannung. Der Sauerstoffgehalt betrug $6 \pm 1$, $51 \pm 2$ und $86 \pm 3$ Prozent und die Temperatur $22 \pm 2{ }^{\circ} \mathrm{C}$. Die angepassten Kurven verlaufen proportional zu $U_{\mathrm{el}}^{4}$. 
90\% Gasgehalt folgen die Werte einem Verlauf proportional zu $U_{\mathrm{el}}^{5}$. Die Werte bei $50 \%$ Gasgehalt folgen vor und mit Überschreiten der Kavitationsschwelle einem Verlauf proportional zu $U_{\mathrm{el}}^{10}$.

\section{Untersuchungen bei $30^{\circ} \mathrm{C}$}

Die kinetische Energiedichte bei $30{ }^{\circ} \mathrm{C}$ und 2 bar Überdruck ist in Abbildung 4.36 aufgetragen. Die Werte für 50\% Gasgehalt liegen am höchsten. Allerdings ist bei dieser Messung die Streuung der Werte groß. So zeigt die durch die Messpunkte gelegte Kurve für 90\% Gasgehalt aufgrund des letzten Messpunktes einen wesentlich flacheren Anstieg als durch die restlichen Punkte vorgegeben. In der logarithmischen Auftragung (Abbildung 4.36(b)) ist wieder ein Anstieg mit leicht abnehmender Steigung zu erkennen. In Abbildung 4.37 sind die Messungen bei $30^{\circ} \mathrm{C}$ und Normaldruck gezeigt. Bei diesen Messungen weisen die Werte bei allen Gasgehalten eine starke Streuung auf. Der Sprung der Messwerte bei 5\% Gasgehalt zeigt den Sprung über die Kavitationsschwelle zwischen 500 und $550 \mathrm{~V}$. Bei $50 \%$ Gasgehalt scheint dieser Sprung zwischen 300 und $350 \mathrm{~V}$ zu liegen. Allerdings zeigt die Aufnahme im Durchlicht bei $200 \mathrm{~V}$ bereits Kavitation, die bei $300 \mathrm{~V}$ nicht auftritt. Der niedrige Messwert bei $200 \mathrm{~V}$ könnte an der Wahl des Messfensters liegen, das den Bereich mit Kavitation direkt an der Sonotrode eventuell ausblendet und somit zu niedrige Werte ergibt. Bei 90\% Gasgehalt liegen die Messwerte alle über der Kavitationsschwelle.

\subsubsection{Vergleich der Energien}

Im folgenden Kapitel werden die Energien im Schallfeld, in der Flüssigkeitsströmung und im vorhandenen Blasenvolumen abgeschätzt und miteinander verglichen. Die Energie im Schallfeld ergibt sich aus der gemessenen Sonotrodenauslenkung bei der jeweiligen Temperatur und der entsprechenden Spannung, wie sie in Kapitel 4.1.3 aufgetragen ist. Mit Hilfe der Formel für die gesamte von einem Kolbenstrahler abgestrahlte Leistung (siehe [100]) lässt sich näherungsweise die von der Sonotrode während zehn Schwingungsperioden abgestrahlte Energie berechnen. Die eingetragenen Fehlerbalken wurden mit Hilfe der Fehlerfortpflanzung aus der Standardabweichung der Sonotrodenauslenkung bestimmt.

Um die im Blasenvolumen gespeicherte Energie abschätzen zu können, wurden die Größenverteilung der Kavitationsblasen anhand eines Beispiels abgeschätzt und in drei Größen im maximal aufgeschwungenen Zustand aufgeteilt. Das gesamte Blasenvolumen wurde berechnet, indem diese Häufigkeitsverteilung auf die Differenz der schwarzen Pixel zwischen aufgeschwungenem und kollabiertem Zustand der Blasen angewendet wurde. Dazu wurde die Häufigkeitsverteilung der Blasen 


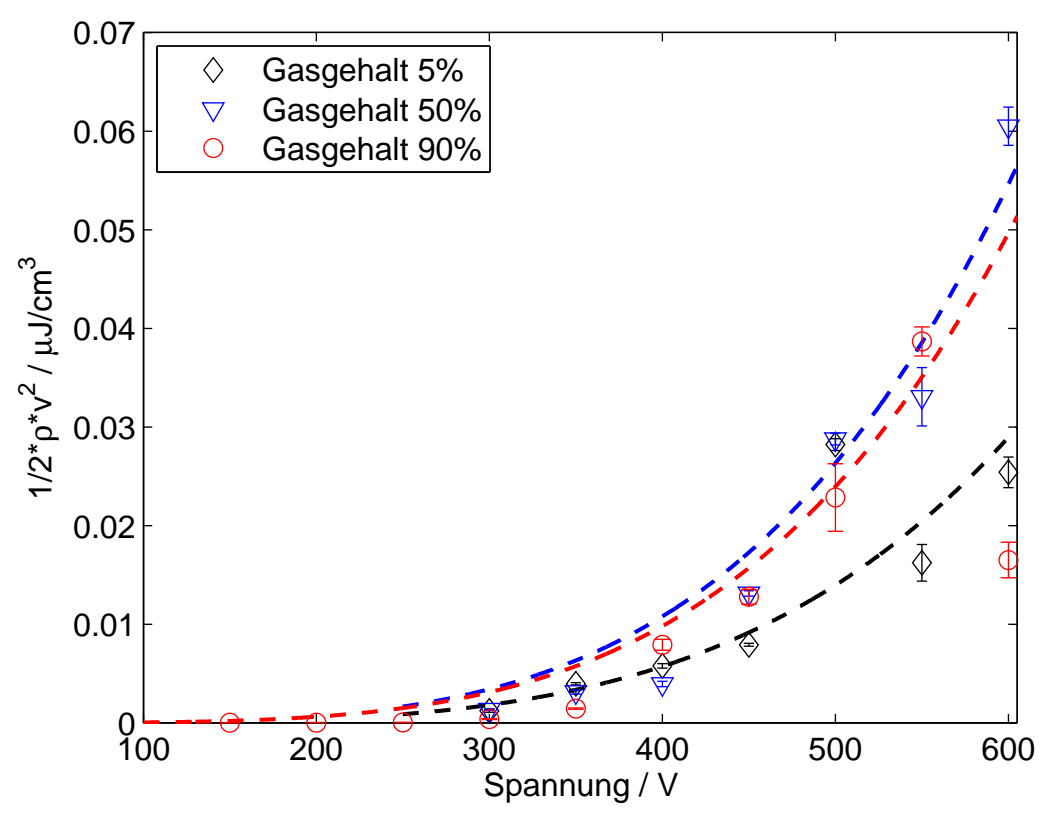

(a) lineare Auftragung

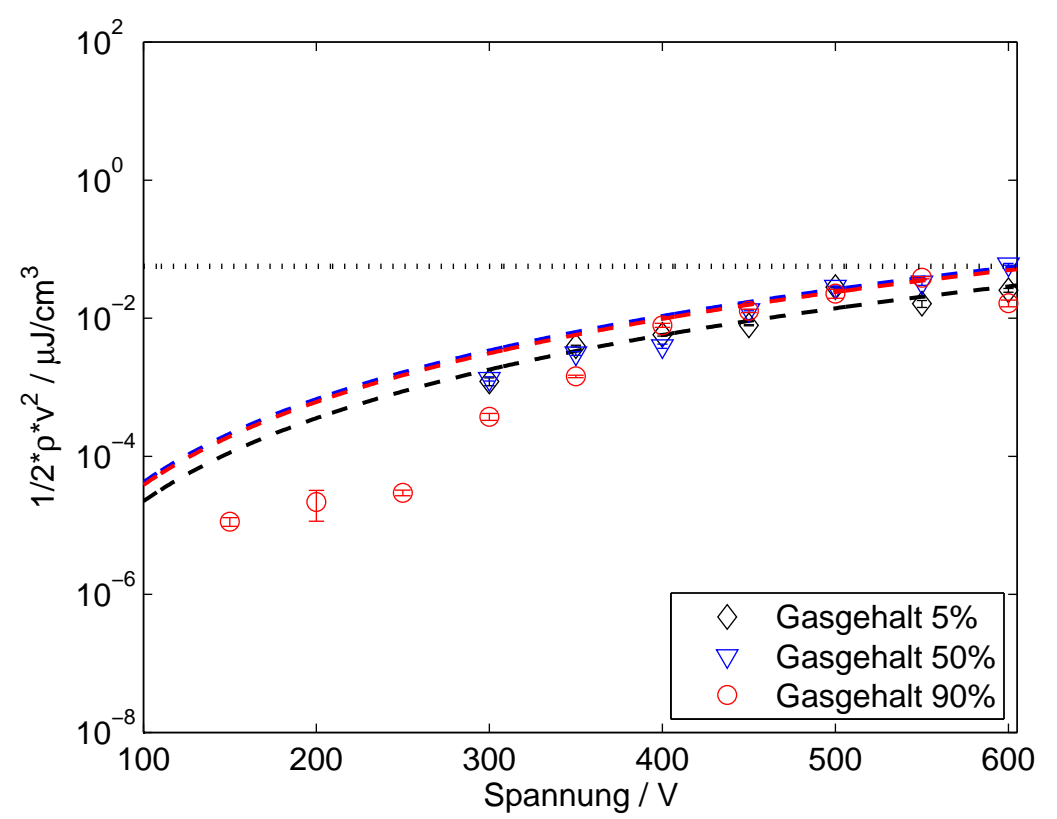

(b) logarithmische Auftragung

Abbildung 4.36.: Kinetische Energiedichte aufgetragen über der an der Sonotrode anliegenden Spannung. Der Sauerstoffgehalt betrug $6 \pm 1$, $51 \pm 1$ und $86 \pm 3$ Prozent und die Temperatur $31 \pm 2{ }^{\circ} \mathrm{C}$. Durch Anlegen von 2 bar Überdruck wurde die Kavitation unterdrückt. Die angepassten Kurven verlaufen proportional zu $U_{\mathrm{el}}^{4}$. 


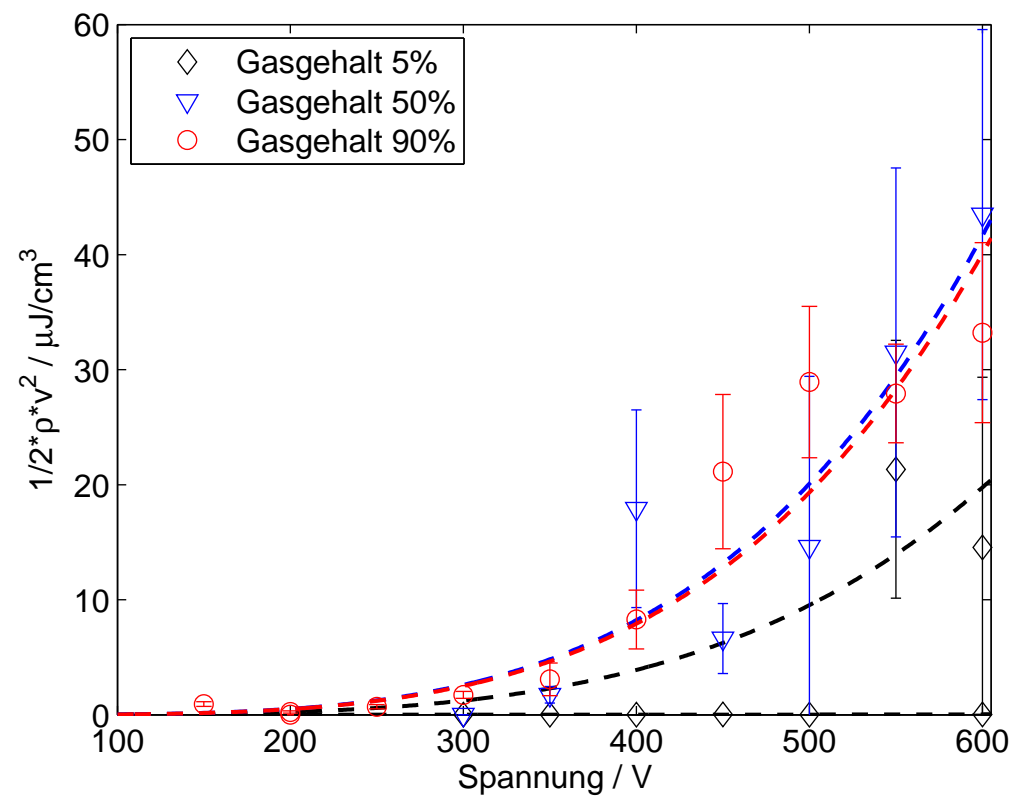

(a) lineare Auftragung

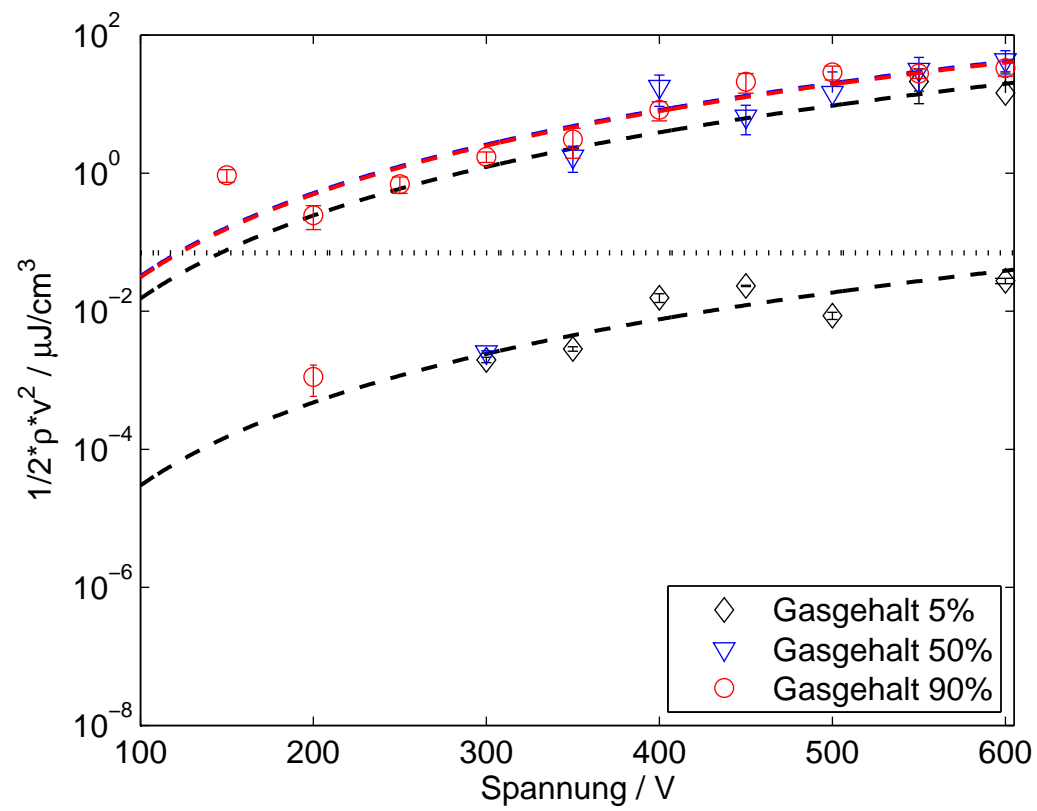

(b) logarithmische Auftragung

Abbildung 4.37.: Kinetische Energiedichte aufgetragen über der an der Sonotrode anliegenden Spannung. Der Sauerstoffgehalt betrug $6 \pm 1$, $51 \pm 1$ und $86 \pm 3$ Prozent und die Temperatur $31 \pm 2{ }^{\circ} \mathrm{C}$. Die angepassten Kurven verlaufen proportional zu $U_{\mathrm{el}}^{4}$. 
in eine prozentuale Verteilung der von den jeweiligen Blasen in einer Projektion abgeschatteten Fläche umgerechnet. Diese wurde anschließend mit der Zahl der schwarzen Pixel korreliert und aus der resultierenden Zahl der Blasen wurde das Volumen bestimmt. Aus dem vorhandenen Blasenvolumen ergibt sich mit Hilfe des statischen Drucks die gespeicherte Energie über die Formel $E_{\mathrm{Vol}}=V p_{0}$. Hier wurden keine Fehlerbalken eingetragen, weil sich der Fehler aufgrund der Annahmen nur schwer abschätzen lässt und als groß angenommen werden kann. Entscheidend ist der qualitative Verlauf, die absoluten Werte sind mit Vorsicht zu betrachten.

Die Geschwindigkeitsvektoren aus der PIV-Software liegen in Matrix-Schreibweise vor. Die kinetische Energiedichte wurde aus dieser Matrix der Geschwindigkeitsvektoren spaltenweise berechnet und jede Spalte mit dem jeweiligen räumlichen Abstand von der Position des Maximums der Energiedichte gewichtet. Es wurde also eine um das Maximum rotationssymmetrische Strömung angenommen. Hier wurden die eingetragenen Fehlerbalken wiederum mit Hilfe der Fehlerfortpflanzungsformel aus der Standardabweichung der gemessenen maximalen Geschwindigkeiten bestimmt. Der Fehler, der sich durch die Unsymmetrie der Strömungsfelder ergibt, wurde nicht berücksichtigt, da er sich schwer quantifizieren lässt. Um diesen Fehler einschätzen zu können, wurde zunächst die kinetische Energiedichte spaltenweise aufgetragen.

\section{Spaltenweise Auftragung der kinetischen Energiedichte}

In den Abbildungen 4.38 bis 4.40 ist die kinetische Energiedichte unter der Sonotrode spaltenweise aufgetragen. Hierbei wurde in Teilabbildung (a) immer die Energiedichte bei Normaldruck und in Teilabbildung (b) die Energiedichte bei 2 bar Überdruck aufgetragen. Die eingetragenen Fehlerbalken zeigen in diesen Auftragungen die zeitliche Standardabweichung des Einzelwertes an, sind also ein Maß für die Streuung der Strömungsfeldänderung mit der Zeit.

In Abbildung 4.38 zeigt das Strömungsfeld bei Normaldruck (Teilabbildung 4.38(a)) und niedrigen Spannungen eine symmetrische Ausprägung. Zwischen 400 und $550 \mathrm{~V}$ ist das Strömungsfeld leicht nach rechts gerichtet und bei $600 \mathrm{~V}$ wieder symmetrisch mittig unter der Sonotrode. Die maximale kinetische Energiedichte nimmt mit der Spannung konstant zu. Die Fehlerbalken sind bei allen Messpunkten klein, was auf eine zeitlich konstante Strömung schließen lässt. In der unteren Teilabbildung 4.38(b) nimmt die Strömung ebenfalls mit der Spannung zu. Das Strömungsfeld ist bei den niedrigen Spannungen leicht nach rechts verschoben, bei den höheren aber symmetrisch ausgeprägt und weist kleine Fehlerbalken auf (siehe Abbildungen 4.14 und 4.23). 
Bei $20^{\circ} \mathrm{C}$ und $90 \%$ Gasgehalt ist das Strömungsfeld bei Normaldruck mit Kavitation (siehe Abbildungen 4.39(a) und auch 4.26) symmetrisch und mittig unter der Sonotrode nach unten gerichtet. Die kinetische Energiedichte nimmt mit der Spannung konstant zu und ist bei $600 \mathrm{~V}$ mit $140 \mu \mathrm{J} \mathrm{cm}^{-3}$ recht hoch. In der unteren Teilabbildung 4.39(b) (siehe auch Abbildung 4.17) ist das Strömungsfeld bei den niedrigen Spannungen (bis $400 \mathrm{~V}$ ) symmetrisch und mittig, die kinetische Energiedichte nimmt konstant zu. Bei 550 und $600 \mathrm{~V}$ ist die Strömung nach rechts gerichtet und bei $550 \mathrm{~V}$ zudem noch niedriger als bei $500 \mathrm{~V}$.

Abbildung 4.40 zeigt die kinetische Energiedichte bei $20^{\circ} \mathrm{C}$ und $50 \%$ Gasgehalt bei Normaldruck (Teilabbildung 4.40(a) und auch Abbildung 4.25) und 2 bar Überdruck (Teilabbildung 4.40(b) und auch Abbildung 4.16). Die Strömungen bei Normaldruck sind größtenteils etwas nach links gerichtet, nehmen aber bis $550 \mathrm{~V}$ konstant zu. Die maximale kinetische Energiedichte ist hier etwa $155 \mathrm{\mu} \mathrm{cm}^{-3}$. Bei $600 \mathrm{~V}$ nimmt die Energiedichte wieder etwas ab und liegt im Maximum nur noch bei $100 \mu \mathrm{J} \mathrm{cm}^{-3}$. Bei 2 bar Überdruck (Teilabbildung 4.40(b)) setzt bei $450 \mathrm{~V}$ Kavitation ein. Dies ist deutlich an der hohen Energiedichte zu erkennen. Die Strömung geht mittig unter der Sonotrode nach unten und ist schmal ausgeprägt.

In einigen dieser Beispiele ist das Strömungsfeld symmetrisch und die Rotationssymmetrie lässt sich ohne großen Fehler anwenden. Teilweise weist das Strömungsfeld allerdings auch eine starke unsymmetrische Ausprägung auf, was den Fehler beim Berechnen der kinetischen Gesamtenergie relativ hoch werden lässt.

\section{Energievergleich}

Von den drei betrachteten Energien ist die durch den Kolbenstrahler innerhalb von zehn Perioden umgesetzte, also die gesamte in dieser Zeit durch die Sonotrode in das Wasser übertragene, Energie am höchsten. Dies verdeutlicht ein Vergleich der in Abbildung 4.41 aufgetragenen Energien. Die Messwerte der Kolbenstrahlerenergie zeigen einen quadratischen Verlauf, eingetragene Fehlerbalken sind relativ groß, was sich auch in Abbildung 4.3 wiederfindet. Die Werte der Volumenenergie schwanken stark, folgen aber im Mittel ebenfalls einem quadratischen Verlauf. Der Verlauf der Energie des Kolbenstrahlers entspricht der Theorie, da die Energie proportional zur eingekoppelten Leistung verläuft. Auch der Verlauf der Volumenenergie entspricht den Erwartungen, da die in den Blasen gespeicherte Energie proportional mit der eingekoppelten Energie steigen sollte. Die Schwankungen und Sprünge der Volumenenergie korrelieren mit der Dauer der einzelnen Messung. Bei den Werten, die über der angepassten Kurve liegen, 


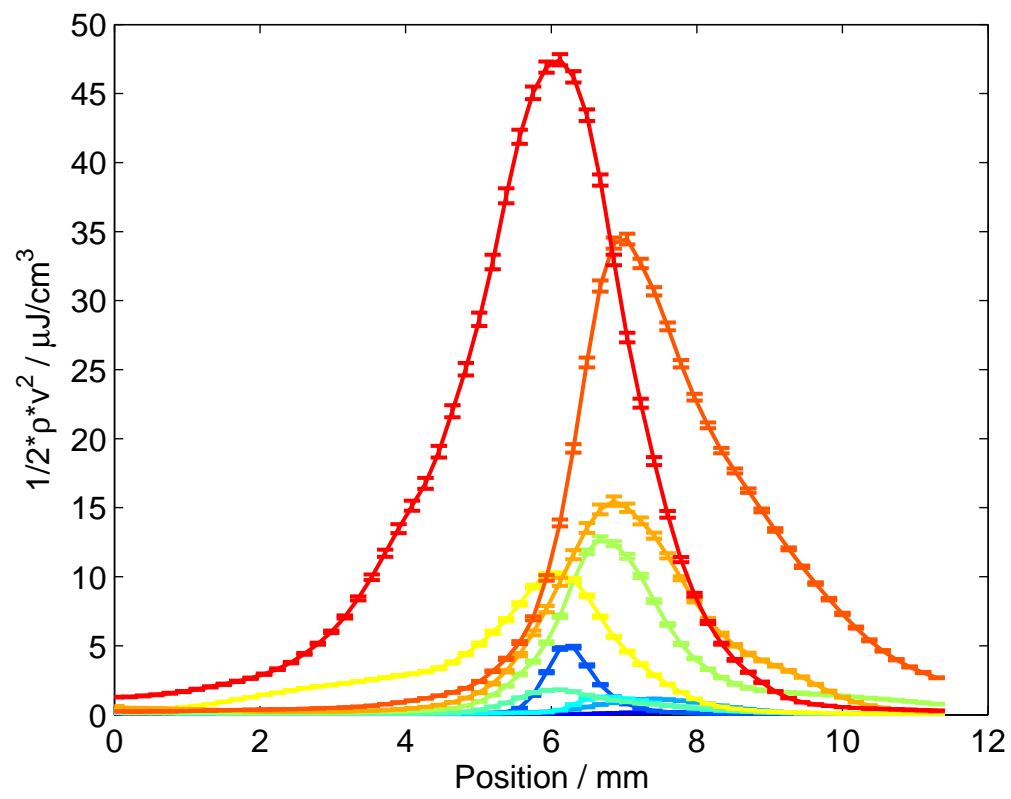

(a) In diesem Beispiel geht das Strömungsfeld mittig unter der Sonotrode nach unten. Zwischen 400 und $550 \mathrm{~V}$ ist es leicht nach rechts gerichtet (siehe auch Abbildung 4.23).

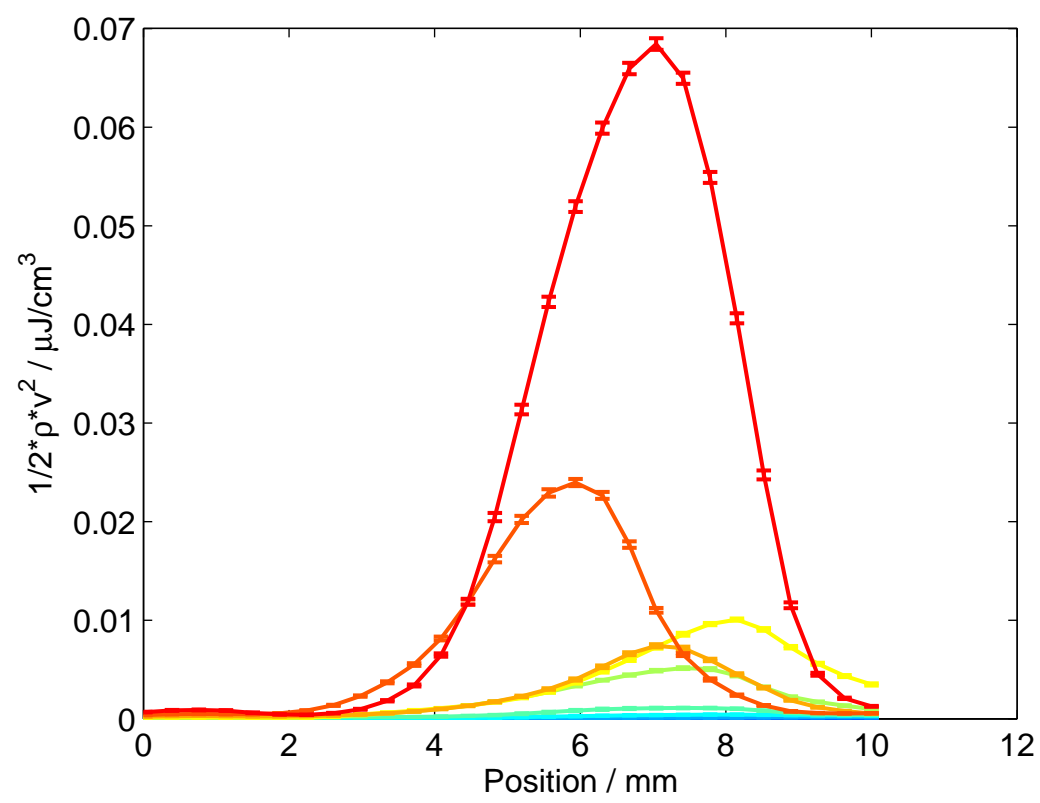

(b) In diesem Beispiel geht das Strömungsfeld bei niedrigen Spannungen leicht nach rechts und bei höheren mittig unter der Sonotrode nach unten (siehe auch Abbildung 4.14).

Abbildung 4.38.: Mittlere kinetische Energiedichte bei $10{ }^{\circ} \mathrm{C}$ und $90 \%$ Gasgehalt von links nach rechts unter der Sonotrode spaltenweise von 150 (blau) bis $600 \mathrm{~V}$ (rot) aufgetragen. 


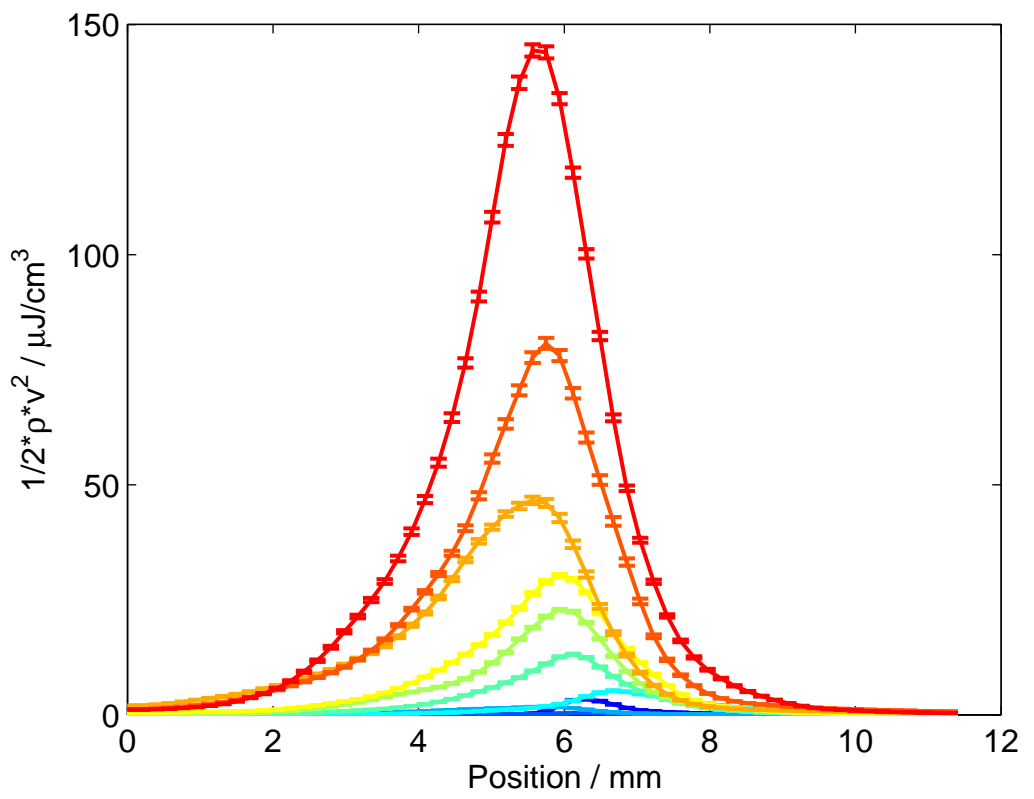

(a) In diesem Beispiel geht das Strömungsfeld mittig unter der Sonotrode nach unten (siehe auch Abbildung 4.26).

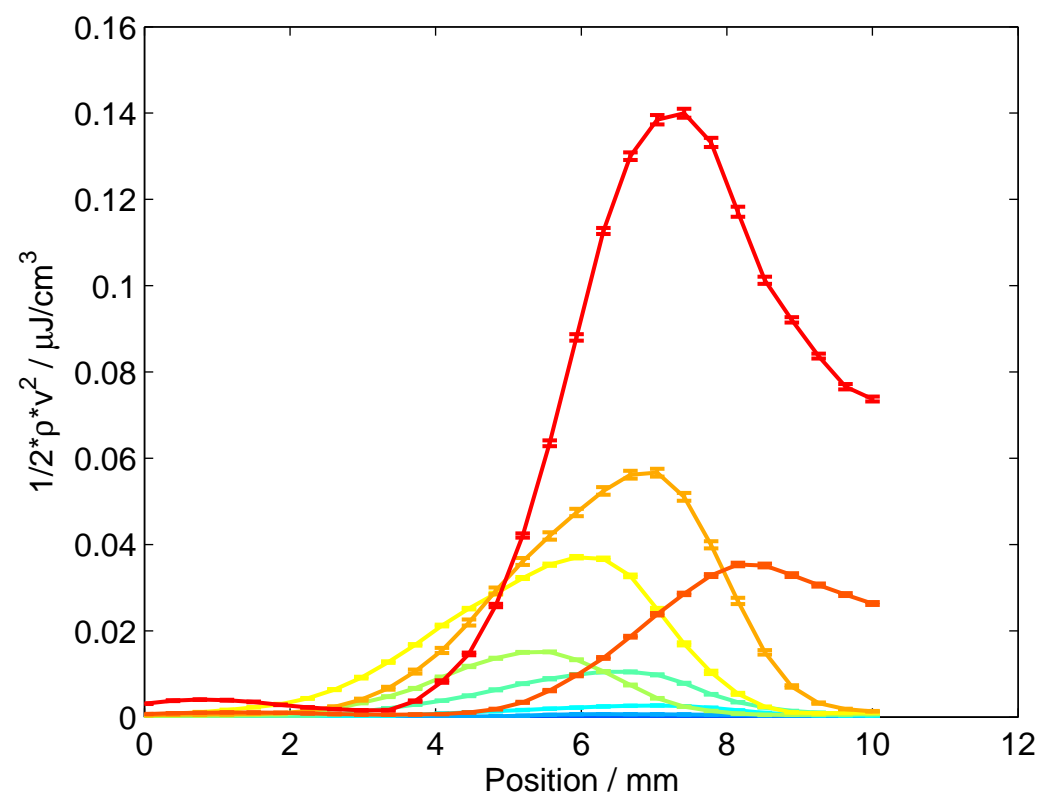

(b) In diesem Beispiel geht das Strömungsfeld unter der Sonotrode leicht nach rechts (siehe auch Abbildung 4.17).

Abbildung 4.39.: Mittlere kinetische Energiedichte bei $20^{\circ} \mathrm{C}$ und $90 \%$ Gasgehalt von links nach rechts unter der Sonotrode spaltenweise von 150 (blau) bis $600 \mathrm{~V}$ (rot) aufgetragen. 


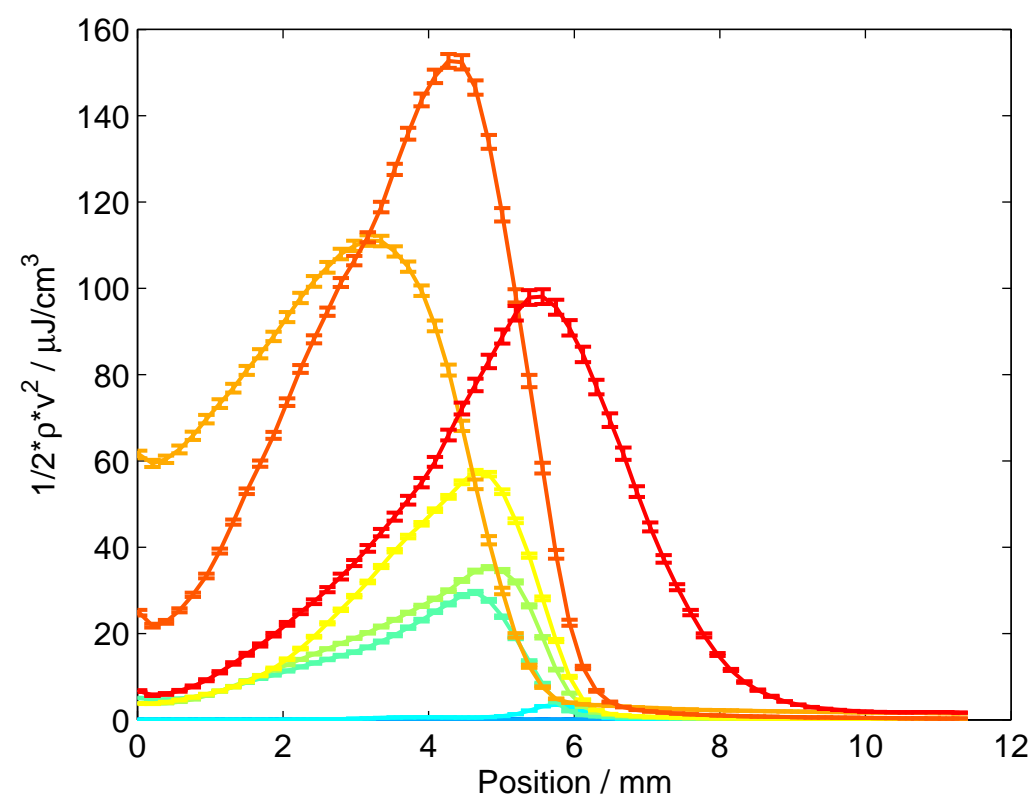

(a) In diesem Beispiel geht das Strömungsfeld unter der Sonotrode nach links (siehe auch Abbildung 4.25).

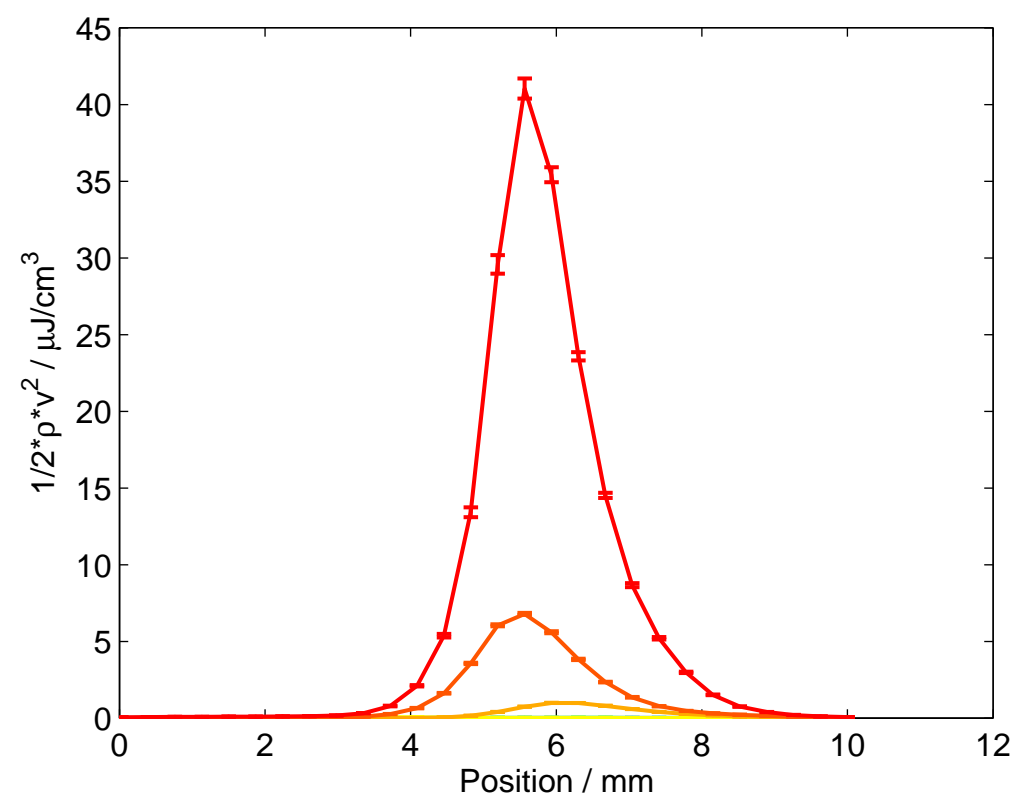

(b) In diesem Beispiel geht das Strömungsfeld mittig unter der Sonotrode nach unten. Bei $450 \mathrm{~V}$ setzt Kavitation ein (siehe auch Abbildung 4.16).

Abbildung 4.40.: Mittlere kinetische Energiedichte bei $20^{\circ} \mathrm{C}$ und $50 \%$ Gasgehalt von links nach rechts unter der Sonotrode spaltenweise von 150 (blau) bis $600 \mathrm{~V}$ (rot) aufgetragen. 


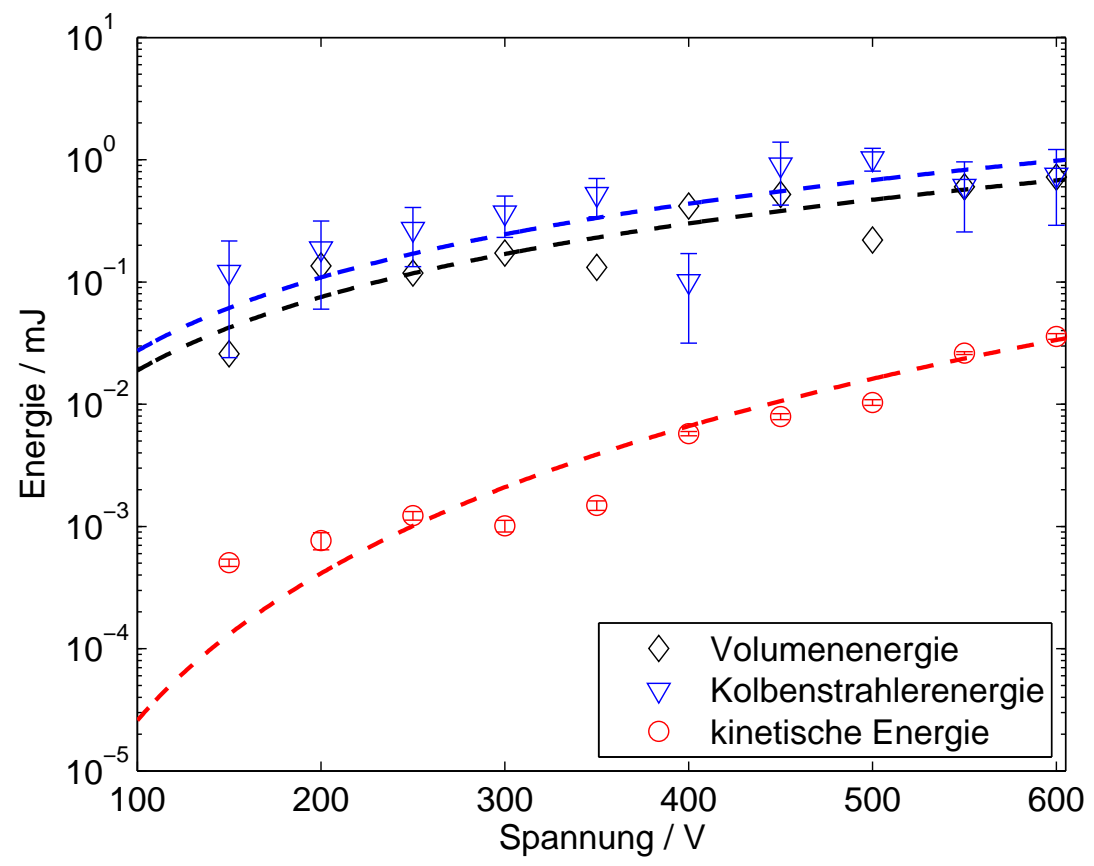

Abbildung 4.41.: Vergleich der gespeicherten Energien bei $10^{\circ} \mathrm{C}$ und $90 \%$ Gasgehalt.

dauerte die Messung relativ lange, bei den Werten darunter wurde die Messung in kurzer Zeit durchgeführt. Dies könnte an einer leichten Temperaturerhöhung während der Messung und somit einem höheren Dampfdruck liegen. Dieser erhöhte Dampfdruck lässt die Blasen größer aufschwingen und führt so zu einem größeren Blasenvolumen im maximal aufgeschwungenen Zustand. Die Werte der kinetischen Energie wurden durch eine Kurve proportional zu $U_{\text {el }}^{4}$ approximiert, der diese im Trend auch folgen. Der stufige Verlauf der kinetischen Energie lässt sich leider nicht erklären. Der einzige Sprung in den Messwerten, der mit einem Messeffekt korreliert, ist der Sprung von $500 \mathrm{zu} 550$ V. Hier wurden beide Messungen ohne Austausch des Wassers durchgeführt, was zu einem größeren Blasenvolumen und somit höherer Geschwindigkeit führen könnte.

In Abbildung 4.42 sind die Energien bei $20^{\circ} \mathrm{C}$ und $90 \%$ Gasgehalt im Vergleich aufgetragen. Wie in der vorherigen Messung ist die Kolbenstrahlerenergie am höchsten, zeigt aber hier geringe Fehlerbalken. Der quadratische Verlauf ist gut zu erkennen. Die berechneten Werte der Volumenenergie streuen bei geringen Spannungen stark, liegen dann aber nahe an der angepassten, zu $U_{\text {el }}^{2}$ proportionalen Kurve. Die Volumenenergie ist wieder leicht geringer als die Kolbenstrahlerenergie. Die kinetische Energie zeigt in diesem Beispiel nur geringe Sprünge, die sich 


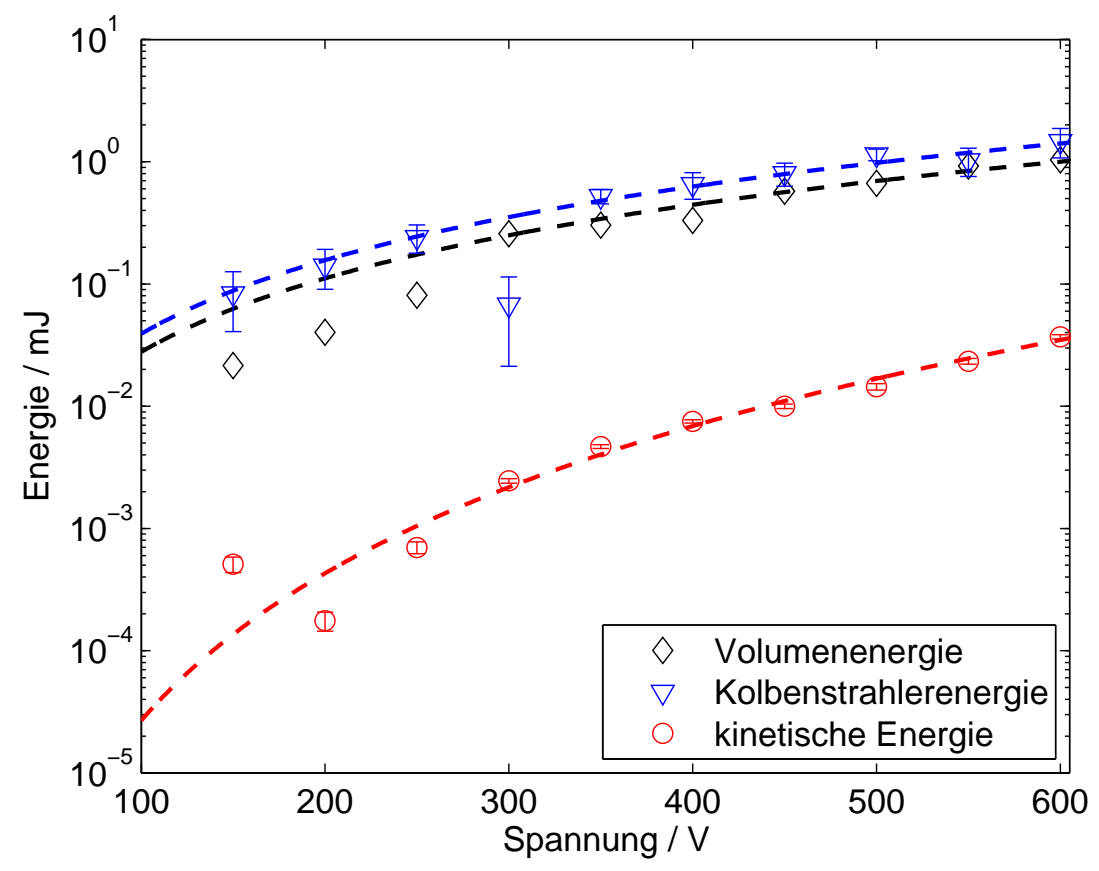

Abbildung 4.42.: Vergleich der gespeicherten Energien bei $20^{\circ} \mathrm{C}$ und $90 \%$ Gasgehalt.

aber hier alle mit den Versuchsbedingungen erklären lassen. So wurden jeweils die Messungen bei 150-250 V, 300-400 V und 450-550 V ohne Austausch des Wassers durchgeführt. Die Messwerte folgen in diesem Fall der angepassten $U_{\mathrm{el}}^{4}$-Kurve.

Bei $20^{\circ} \mathrm{C}$ und $50 \%$ Gasgehalt (Abbildung 4.43) wird die Kavitationsschwelle erst bei $200 \mathrm{~V}$ überschritten. Der berechnete Wert der Volumenenergie bei $150 \mathrm{~V}$ zeigt also das Grundrauschen an. Die folgenden Werte zeigen genau wie die Werte der kinetischen Energie einen stärkeren Anstieg, um dann bei $350 \mathrm{~V}$ jeweils die angepasste Kurve zu erreichen. Dieser folgen sie dann bei den Werten der Volumenenergie mit $U_{\mathrm{el}}^{2}$ und nur einer größeren Abweichung und bei den Werten der kinetischen Energie ohne Abweichungen mit $U_{\mathrm{el}}^{4}$.

Um den Sprung über die Kavitationsschwelle noch einmal zu verdeutlichen wurde außerdem in Abbildung 4.44 der Vergleich der Energien für $20^{\circ} \mathrm{C}$ und $5 \%$ Gasgehalt aufgetragen. Hier erfolgt das Erreichen der Kavitationsschwelle erst bei 450 V. Unterhalb der Schwelle ist anhand der Werte der Volumenenergie wieder das Grundrauschen dieser Methode zu erkennen. Die Zunahme der Messwerte über das Grundrauschen ist etwas mehr als eine Größenordnung, die kinetische Energiedichte nimmt um etwas mehr als drei Größenordnungen zu. 


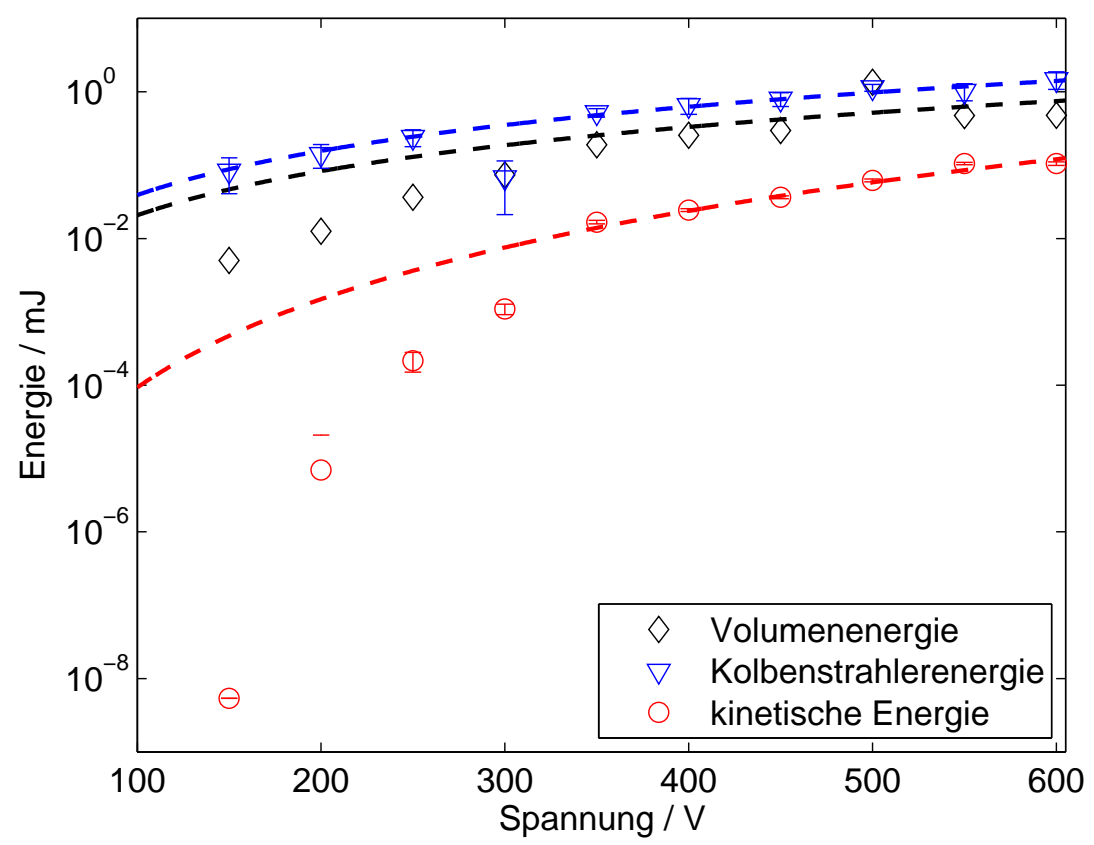

Abbildung 4.43.: Vergleich der gespeicherten Energien bei $20^{\circ} \mathrm{C}$ und $50 \%$ Gasgehalt.

\subsection{Charakterisierung der Strömung}

Um die Art der Strömung genauer bestimmen zu können, wurden verschiedene Methoden angewendet. Zur Charakterisierung der Turbulenz einer Strömung kann eine Untersuchung der Korrelation herangezogen werden [101]. So weisen laminare Strömungen ein hohes Maß an Korrelation auf, da sich die Strömung mit der Zeit kaum oder periodisch ändert. Turbulente Strömungen hingegen sind in hohem Maße unkorreliert.

Eine weitere Kennzahl von Strömungen ist die Reynolds-Zahl. Diese Zahl setzt die Geschwindigkeit einer Strömung mit einer Längenskala in Verbindung. Bei umströmten Körpern in Flüssigkeiten ist dies für gewöhnlich eine Abmessung des Körpers. Bei Rohrströmungen wird der Durchmesser des Rohres zur Berechnung herangezogen. Je nach Anwendungsfall und Berechnungsmethode tritt der Übergang zwischen laminarer und turbulenter Strömung bei verschiedenen ReynoldsZahlen ein. 


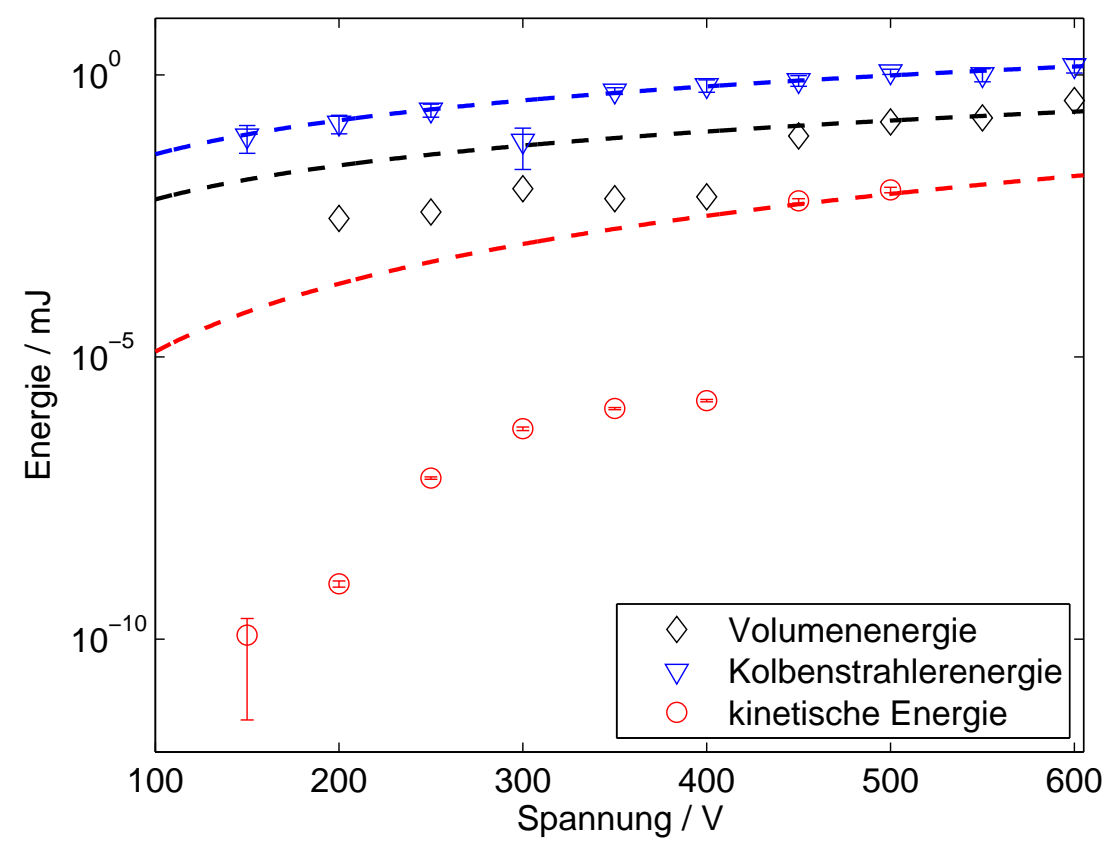

Abbildung 4.44.: Vergleich der gespeicherten Energien bei $20^{\circ} \mathrm{C}$ und $5 \%$ Gasgehalt.

\subsubsection{Zeitliche Korrelation der Strömung}

Zum genaueren Untersuchen der Strömung wurde eine Kreuzkorrelation der Geschwindigkeitskomponenten $u(t)$ und $v(t+\tau)$ in einem kleinen Auswertungsfenster durchgeführt. Dieses Fenster wurde etwa in die Mitte des Gesamtbildes gelegt. In Abbildung 4.45 ist der zeitliche Verlauf der Korrelation bei $10^{\circ} \mathrm{C}$, 90\% Gasgehalt und $600 \mathrm{~V}$ aufgetragen. Die Kreuzkorrelation bei 2 bar Überdruck verläuft größtenteils zwischen 0.8 und 1, das heißt die Geschwindigkeitskomponenten sind stark korreliert. Die Geschwindigkeitsvektoren ändern sich in diesem Fall mit der Zeit kaum, die Strömung ist laminar. Bei Normaldruck schwankt die Korrelation stark zwischen 0.9 und -0.9. In diesem Fall ändert sich also die Richtung der Komponenten zueinander häufig und unkorreliert. Allerdings treten ab und zu auch Phasen starker Korrelation auf. Die Strömung weist immer wieder turbulente Phasen auf, wechselt aber auch häufig zu laminarer Strömung.

Bei $20^{\circ} \mathrm{C}$, 90\% Gasgehalt und $500 \mathrm{~V}$ (Abbildung 4.46) bietet sich ein ähnliches Bild. Genau wie im vorherigen Fall verläuft die Korrelation der Geschwindigkeitskomponenten bei 2 bar Überdruck größtenteils zwischen 0.8 und 1. Die Strömung bei Normaldruck wechselt zwischen hoher Korrelation und einer unkorrelierten Strömung. 


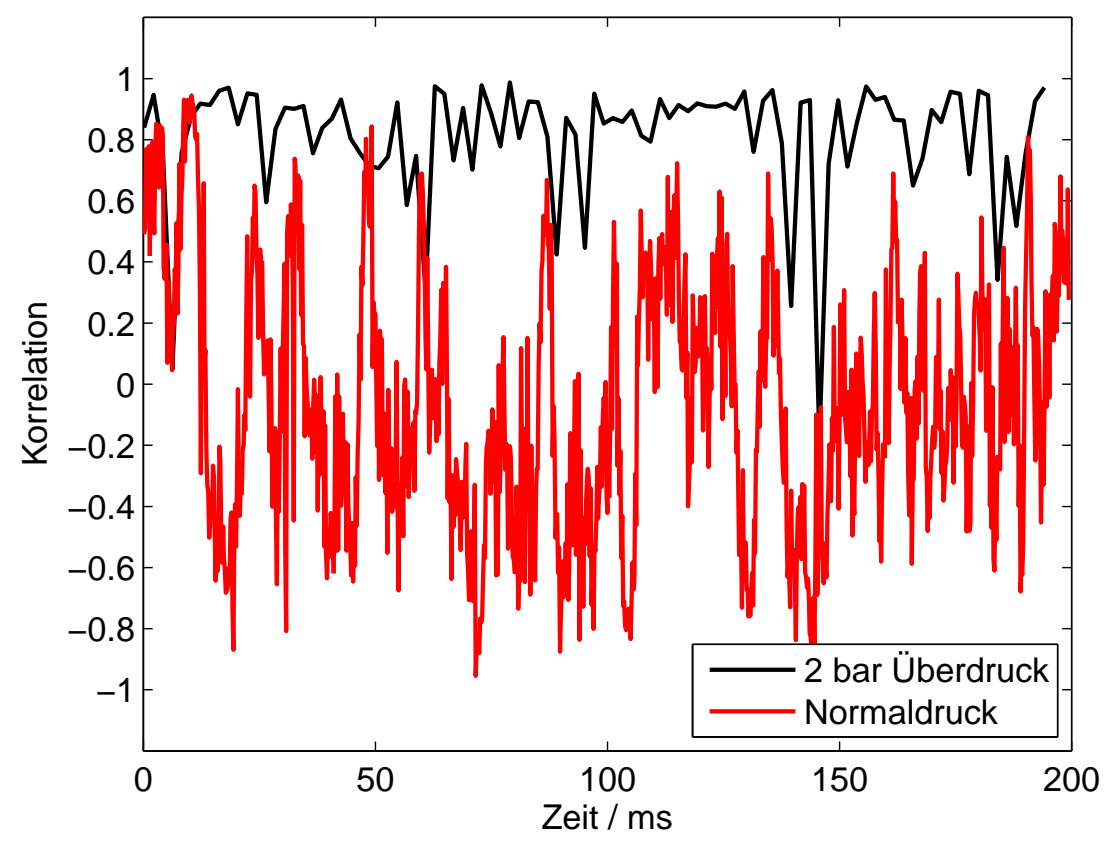

Abbildung 4.45.: Zeitlicher Verlauf der Kreuzkorrelation bei $10{ }^{\circ} \mathrm{C}, 90 \%$ Gasgehalt und $600 \mathrm{~V}$ mit und ohne Kavitation im Vergleich.

Die Korrelation bei $20^{\circ} \mathrm{C}, 90 \%$ Gasgehalt und $500 \mathrm{~V}$ (Abbildung 4.47) zeigt einen anderen Verlauf. Sowohl die Kurve bei Normaldruck, als auch die Kurve bei 2 bar Überdruck schwanken stark und zeigen eine geringe Korrelation. Beim Vergleich der Strömungen für 500 und $600 \mathrm{~V}$ in Abbildung 4.17 fällt auf, dass die Strömung bei $600 \mathrm{~V}$ stark nach rechts gerichtet ist. Das Auswertungsfenster wurde aber in der Bildmitte, also im Bereich geringer Strömung, platziert. Durch die langsame Bewegung der Tracer-Partikel, und im Vergleich dazu hohe Bildrate, treten beim Auswerten der Strömung mit der PIV-Software viele Fehler auf, die sich als Rauschen manifestieren. Dieser Effekt senkt die Korrelation in dem Bereich stark. In Abbildung 4.48 ist die Kreuzkorrelation der Geschwindigkeitskomponenten bei $20^{\circ} \mathrm{C}, 50 \%$ Gasgehalt und $600 \mathrm{~V}$ bei Normal- und Überdruck aufgetragen. Wie in Kapitel 4.3 bereits dargelegt, trat in diesem Fall auch mit Überdruck Kavitation auf. Die Kurven mit und ohne Überdruck verlaufen hier sehr ähnlich und zeigen beide geringe Korrelation, was auf eine Strömung mit einem hohen Grad an Turbulenz schließen lässt. 


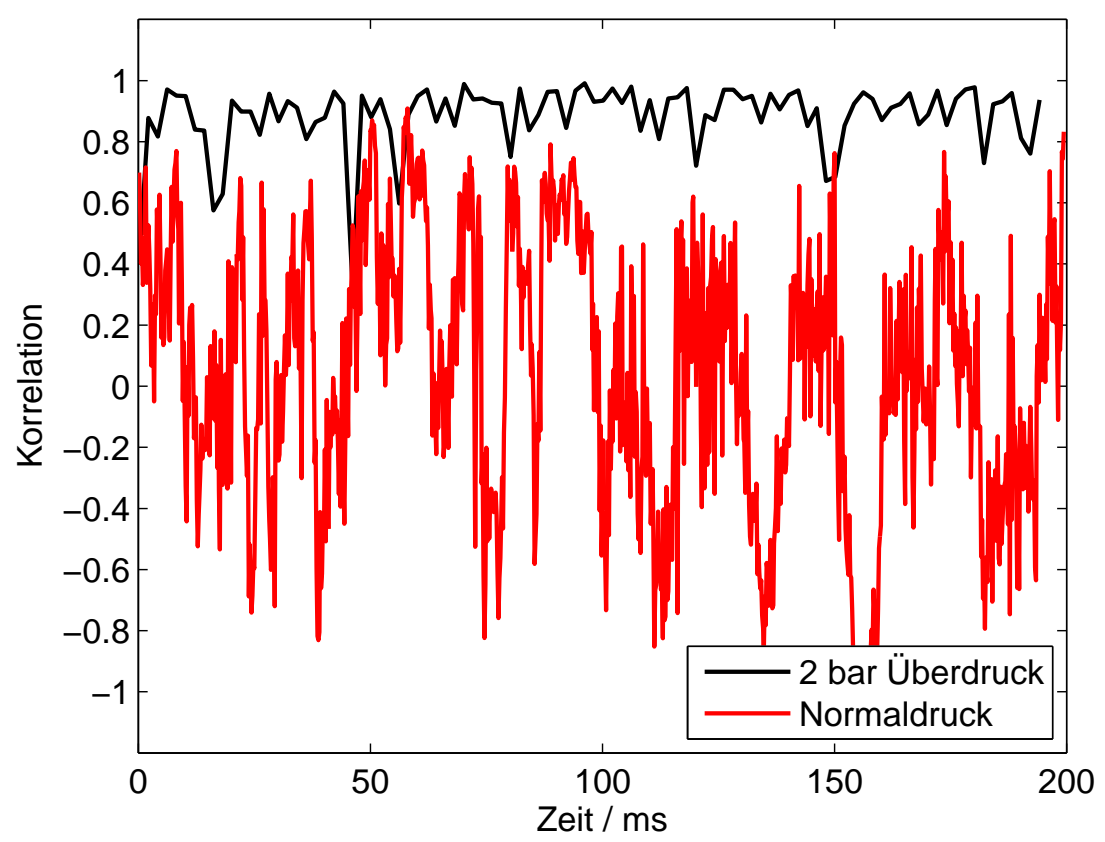

Abbildung 4.46.: Zeitlicher Verlauf der Kreuzkorrelation bei $20^{\circ} \mathrm{C}, 90 \%$ Gasgehalt und $500 \mathrm{~V}$ mit und ohne Kavitation im Vergleich.

\subsubsection{Reynolds-Zahlen mit und ohne Kavitation}

Eine weitere Kennziffer für die Charakterisierung von Strömungen ist die Reynolds-Zahl. Um die Reynolds-Zahl für diesen Fall zu berechnen, wurde die akustische Strömung als freier Jet betrachtet. In dem von AfANASEv [102] betrachteten Fall wird Flüssigkeit durch ein offenes Rohr in ein großes Flüssigkeitsvolumen eingeleitet. Die charakteristische Länge ist dann der Rohrdurchmesser und die Geschwindigkeit $U_{0}$ wird in der Mitte der Rohrströmung am Austritt gemessen. Für den vorliegenden Fall der akustischen Strömung wurde der Durchmesser der Strömung an der schmalsten Stelle bei voller Ausprägung der Strömung gemessen. Auch die Geschwindigkeit wurde an dieser Stelle mittig gemessen, indem über mehrere Werte in Richtung der Strömung und über die Zeit gemittelt wurde, um die Nettoströmung zu erhalten. Außerdem wurde nur die Geschwindigkeitskomponente von der Sonotrode weg betrachtet. Die Entfernung von der Sonotrode und der Durchmesser $d$ der Strömung wurde bei der Berechnung aller Reynolds-Zahlen als konstant angenommen. Die Reynolds-Zahl berechnet sich dann nach der Formel: $R e=d U_{0} / \nu$, mit der kinematischen Viskosität $\nu=\mu / \rho$. Nur die Geschwindigkeit der Strömung wurde aus dem jeweiligen Datensatz ausgelesen. Genau genommen ist der Fall der akustischen Strömungen 


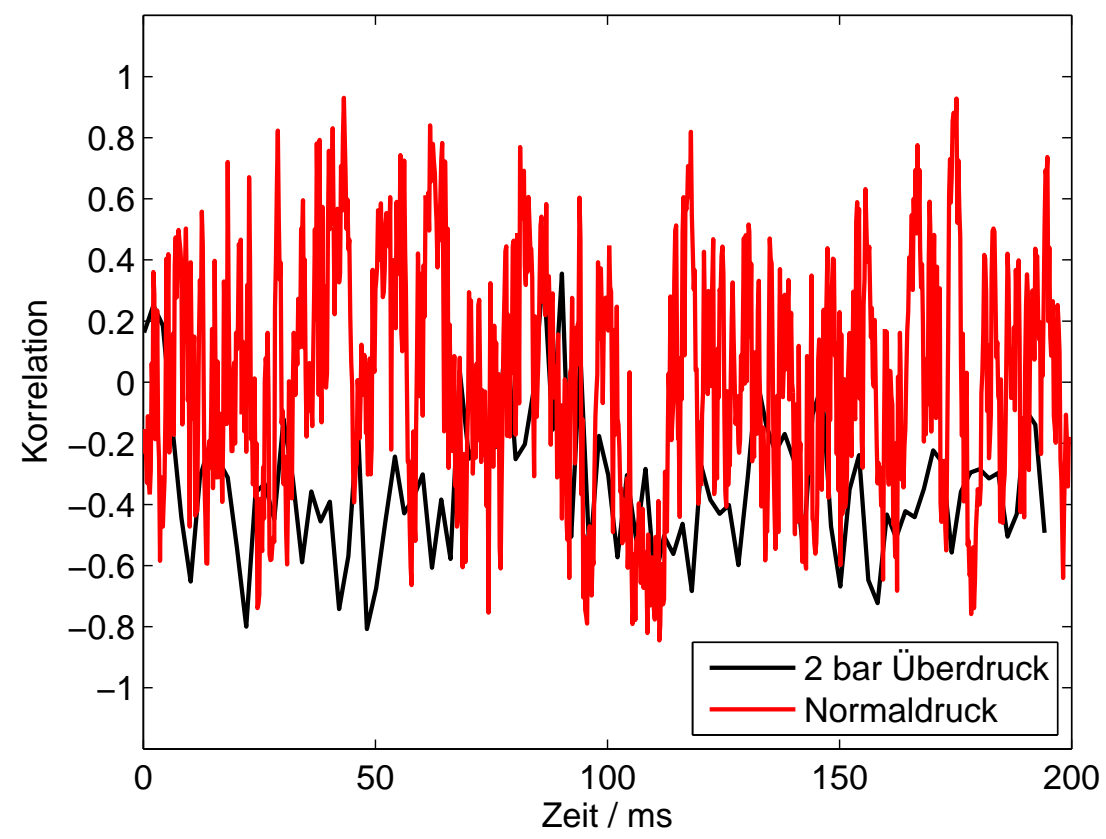

Abbildung 4.47.: Zeitlicher Verlauf der Kreuzkorrelation bei $20^{\circ} \mathrm{C}, 90 \%$ Gasgehalt und $600 \mathrm{~V}$ mit und ohne Kavitation im Vergleich.

niedriger Dämpfung etwas anders, da die Strömung durch die Schallausbreitung und Dämpfung auf ihrer gesamten Länge weiter beschleunigt wird. Allerdings tritt in beiden Fällen, dem freien Jet durch eine Rohrströmung und dem durch akustische Strömung erzeugtem Jet, je nach Durchmesser und Geschwindigkeit an den Rändern des Jets Turbulenz auf. Beim freien Jet wird die Strömung ab Reynolds-Zahlen von etwa 3500 als turbulent bezeichnet. Die Geschwindigkeit der Strömung nimmt, wie in Kapitel 2.1.2 beschrieben, quadratisch mit der Spannung zu. Da die Reynolds-Zahl proportional mit der Geschwindigkeit steigt, sollte sich ein quadratischer Verlauf mit der elektrischen Spannung einstellen.

Die Reynolds-Zahlen für $10^{\circ} \mathrm{C}$ und $50 \%$ Gasgehalt (Abbildung 4.49) sind bei geringen Spannungen niedrig und steigen dann stark an. Bei 2 bar Überdruck wird ab etwa $450 \mathrm{~V}$ ein Verlauf proportional zu $U_{\text {el }}^{2}$ erreicht. Die maximale ReynoldsZahl liegt bei 76. Bei Normaldruck folgen die Reynolds-Zahlen am Anfang dem Verlauf der Zahlen bei 2 bar Überdruck. Bei $300 \mathrm{~V}$ wird die Kavitationsschwelle überschritten und die Reynolds-Zahl steigt sprunghaft auf etwa 200 an. Die maximale Zahl liegt hier bei 2281 und der Verlauf ist etwas steiler als $U_{\mathrm{el}}^{2}$.

Die Reynolds-Zahlen in Abbildung 4.51 bei $20^{\circ} \mathrm{C}$ und $90 \%$ Gasgehalt weisen bei höheren Spannungen einen Verlauf nahe der angepassten Kurve auf. Bei niedrige- 


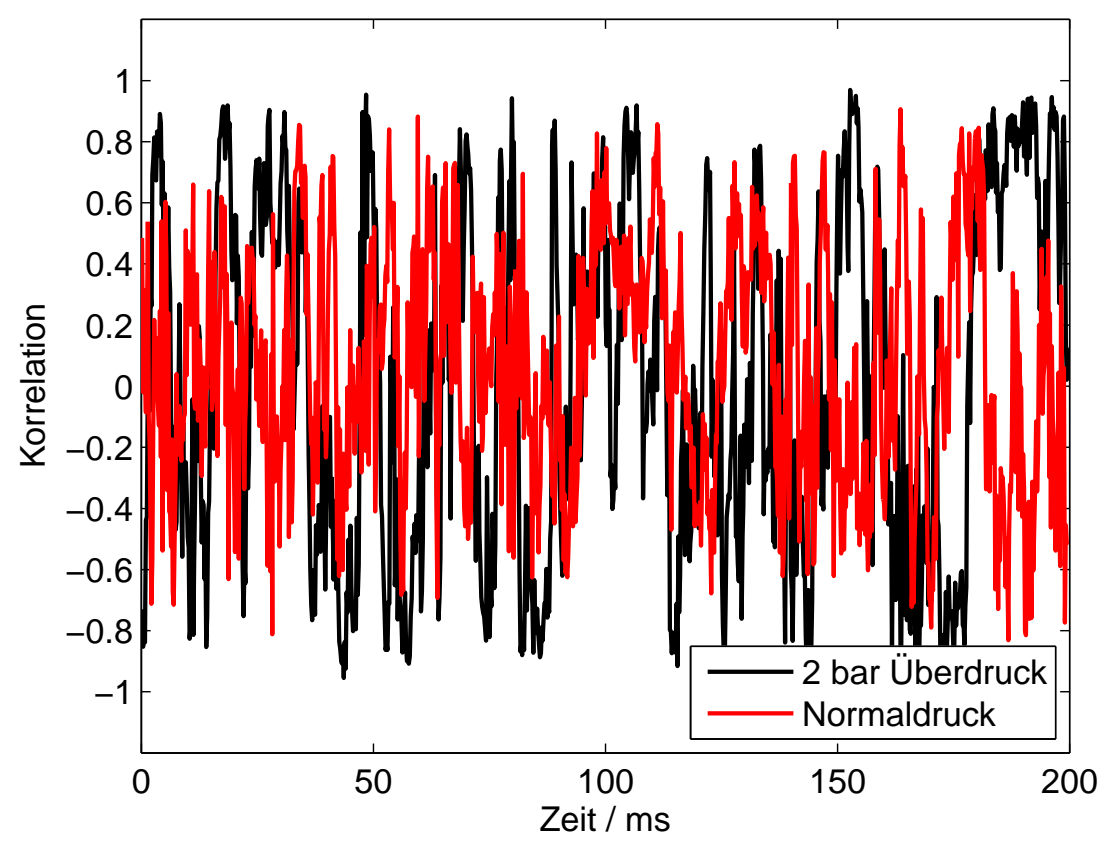

Abbildung 4.48.: Zeitlicher Verlauf der Kreuzkorrelation bei $20^{\circ} \mathrm{C}, 50 \%$ Gasgehalt und $600 \mathrm{~V}$ mit und ohne Überdruck im Vergleich.

ren Spannungen weichen sie stark ab. Die maximale Reynolds-Zahl bei Überdruck ohne Kavitation liegt bei 71. Bei Normaldruck mit Kavitation werden Werte bis 2481 erreicht.

In Abbildung 4.51 bietet sich zu Beginn wieder ein ähnliches Bild, dass die Reynolds-Zahlen stark von der angepassten Kurve abweichen. Bei Überdruck setzt bei einer Spannung von 450 V Kavitation ein und die Reynolds-Zahlen steigen stark an. Bei $600 \mathrm{~V}$ erreichen sie fast die gleichen Werte wie die Strömung bei Normaldruck (1690 zu 2117), bei der die Kavitationsschwelle bei 300 V überschritten wurde. Die maximale Reynolds-Zahl ohne Kavitation ist hier bei 40, mit Kavitation wird bis zu 3350 erreicht. 


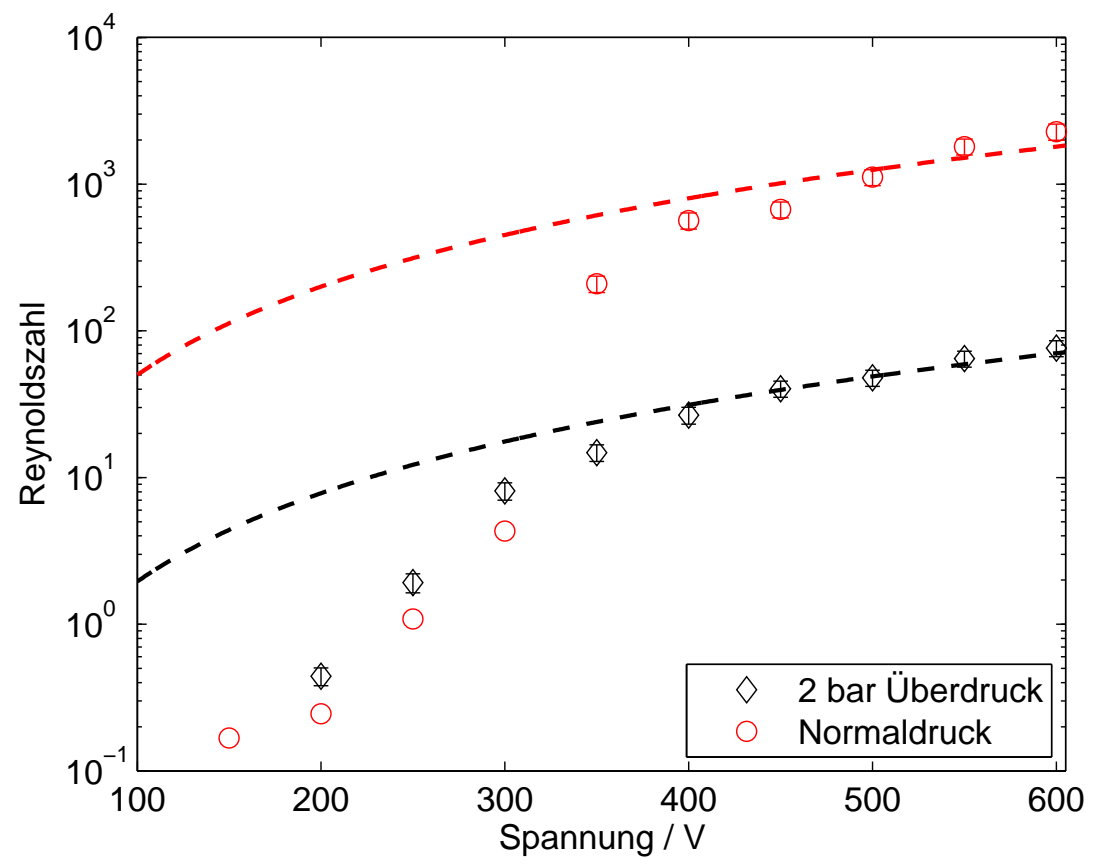

Abbildung 4.49.: Reynolds-Zahl der Strömungen bei $10^{\circ} \mathrm{C}$ und $50 \%$ Gasgehalt mit und ohne Kavitation im Vergleich. Berechnet wurde die Reynolds-Zahl eines freien Jets mit $4 \mathrm{~mm}$ Durchmesser und der jeweiligen Strömungsgeschwindigkeit auf Mittelachse.

\subsubsection{Frequenzspektren der Strömungsgeschwindigkeiten und des Blasenvolumens}

In diesem Kapitel werden anhand der Frequenzspektren der mittleren Geschwindigkeitsamplitude und der Anzahl der schwarzen Pixel verschiedene Aspekte der Strömung an der Sonotrode erläutert. Die Anzahl der schwarzen Pixel in den Durchlichtaufnahmen ist geeignet, um den zeitlichen Verlauf des Schattens durch die Blasen und somit des Blasenvolumens abzuschätzen.

In den Abbildungen 4.52 und 4.53 sind jeweils in schwarz die Amplitude der Fouriertransformierten des zeitlichen Verlaufs der schwarzen Pixel und in rot die Amplitude der Fouriertransformierten des zeitlichen Verlaufs der mittleren Geschwindigkeitsamplitude über der Frequenz aufgetragen. Aufgrund der Aufnahmerate von 5000 Bildern pro Sekunde konnte diese nur bis $2500 \mathrm{~Hz}$ aufgetragen werden (NyQUiST-Frequenz [103]).

In der ersten Abbildung sind die Amplituden bei $20^{\circ} \mathrm{C}, 5 \%$ Gasgehalt und $400 \mathrm{~V}$ dargestellt. Hier trat keine Kavitation auf. Es sind in beiden Fällen deutliche Spitzen bei 2282 und $810 \mathrm{~Hz}$ zu erkennen. Außerdem weisen beide Kurven ei- 


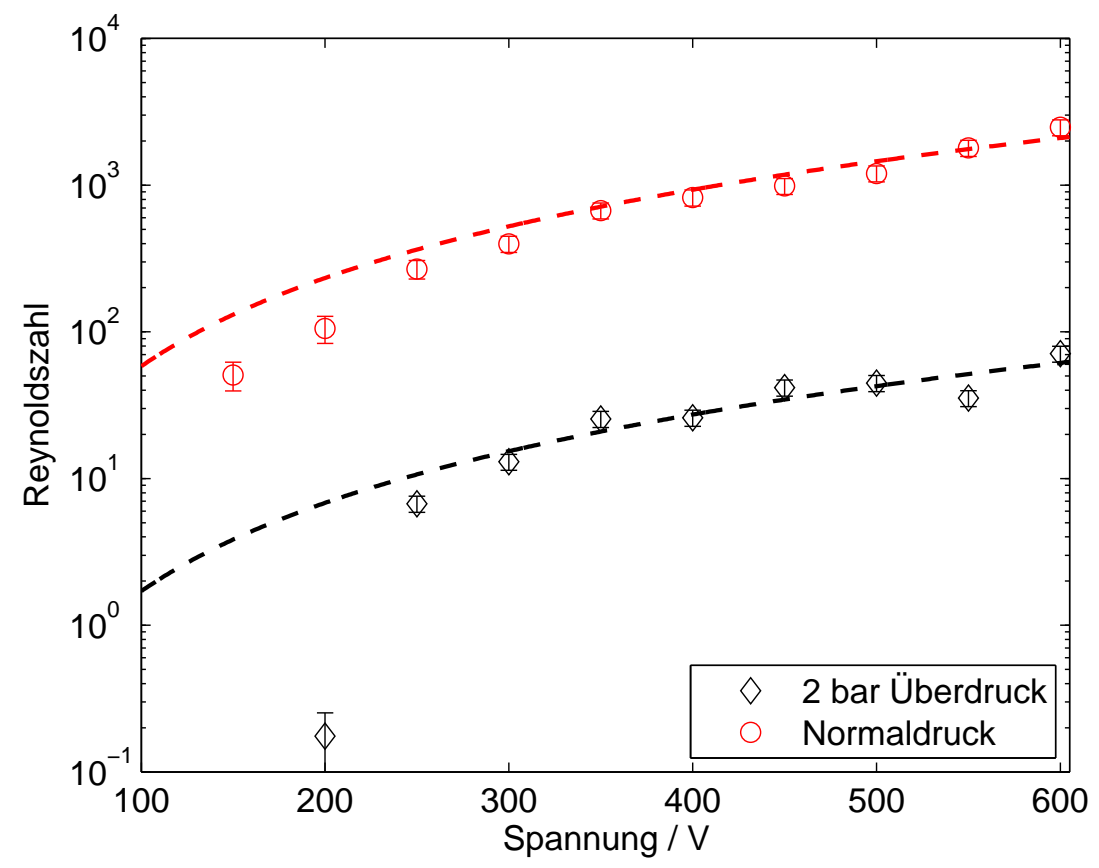

Abbildung 4.50.: Reynolds-Zahl der Strömungen bei $20^{\circ} \mathrm{C}$ und $90 \%$ Gasgehalt mit und ohne Kavitation im Vergleich. Berechnet wurde die Reynolds-Zahl eines freien Jets mit $4 \mathrm{~mm}$ Durchmesser und der jeweiligen Strömungsgeschwindigkeit auf der Mittelachse.

ne weitere Spitze bei $100 \mathrm{~Hz}$ und jeweils weitere kleine Spitzen auf. Die höchste Spitze der Kurve für die Anzahl der schwarzen Pixel korreliert im Rahmen der Abtastgenauigkeit mit der Differenzfrequenz zwischen der Frequenz der Sonotrode und der dreifachen Abtastrate von $15 \mathrm{kHz}$. Auch die Geschwindigkeitsamplitude weist hier eine Spitze auf. Bei der langsamen Strömung ohne Kavitation spielt anscheinend die Phasendifferenz zwischen Anregungsfrequenz und Aufnahmefrequenz eine Rolle, obwohl mit 1/6000s über fast drei Perioden belichtet wurde. In diesem Fall ist die Schwingung der Flüssigkeit durch die Schallschnelle zu erkennen.

In Abbildung 4.53 sind ebenfalls die Fouriertransformierten der mittleren Geschwindigkeitsamplitude und der Anzahl der schwarzen Pixel diesmal für $20^{\circ} \mathrm{C}$, 90\% Gasgehalt und 600 V aufgetragen. Die Fouriertransformierte des zeitlichen Verlaufs des Schwarzwertes weist wieder eine Spitze bei $2285 \mathrm{~Hz}$ auf. Diese Spitze fehlt bei der Geschwindigkeitsamplitude komplett. Es treten auch keine anderen Spitzen auf. In diesem Fall überlagert die sehr viel schnellere Nettoströmung die Schallschnelle und die Phase der Aufnahme spielt keine Rolle mehr. 


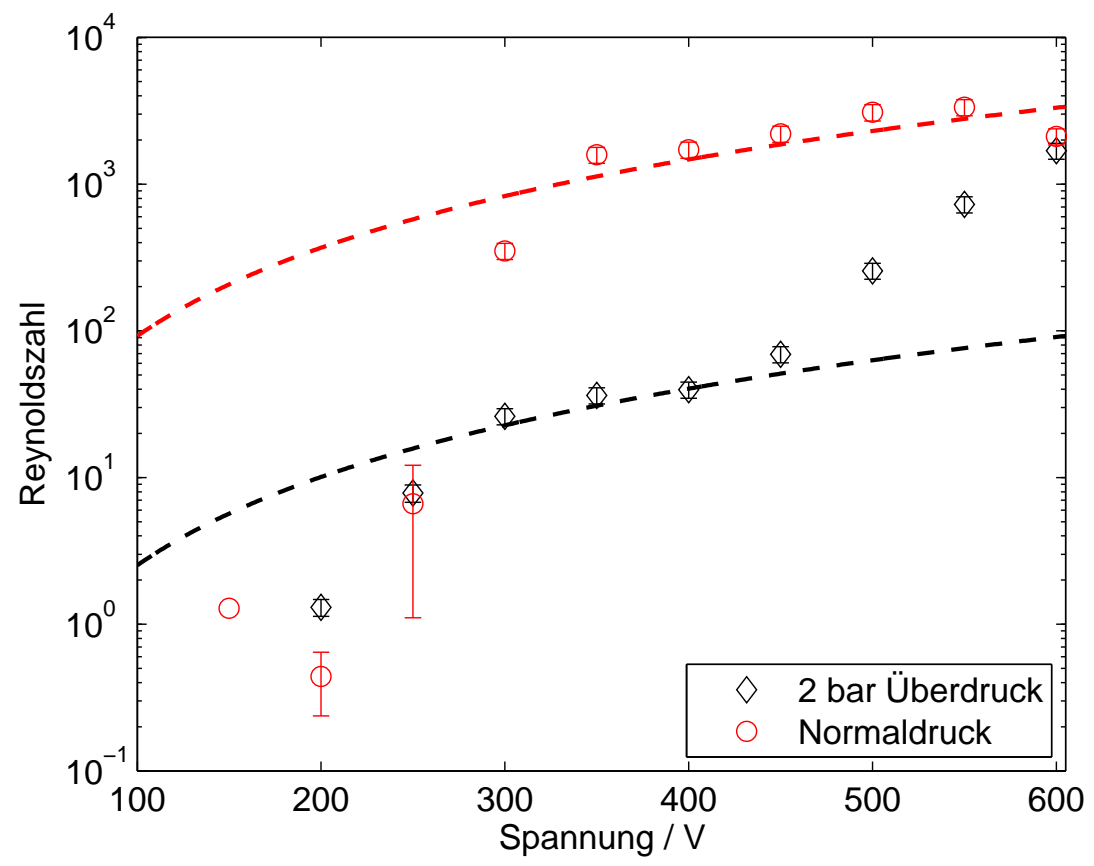

Abbildung 4.51.: Reynolds-Zahl der Strömungen bei $20^{\circ} \mathrm{C}$ und $50 \%$ Gasgehalt mit und ohne Kavitation im Vergleich. Berechnet wurde die Reynolds-Zahl eines freien Jets mit $4 \mathrm{~mm}$ Durchmesser und der jeweiligen Strömungsgeschwindigkeit auf der Mittelachse.

\subsection{Detailaufgelöste Aufnahmen}

Mit 50000 Bildern pro Sekunde wurde ein kleiner Bereich direkt an der Sonotrode bei $20^{\circ} \mathrm{C}, 90 \%$ Gasgehalt und $17.381 \mathrm{kHz}$ aufgenommen. Durch die hohe Aufnahmerate mit etwas mehr als drei Bildern pro Periode ist das Geschwindigkeitsfeld direkt an der Sonotrode zeitaufgelöst während der Blasenschwingungen sichtbar. Eine zeitliche Abfolge von Bildern bei $260 \mathrm{~V}$ ist in Abbildung 4.54 dargestellt. Die Zeit läuft hier zeilenweise von links oben nach rechts unten. Zeitlich kurze Effekte innerhalb einer Anregungsperiode lassen sich hierdurch im Verlauf einer Zeile erkennen. Effekte über eine Periode hinaus sind innerhalb einer Spalte ablesbar. So lässt sich in den Zeilen das Kollabieren und Aufschwingen der Blasen mit der Strömung in Verbindung bringen. Innerhalb einer Spalte sind Bewegungen der Blasen zu beobachten. Es ist zu erkennen, dass sich die Blasen alle auf die Sonotrode zu bewegen. Die Strömungsrichtung wechselt je nachdem zu welcher Phase der Sonotrodenschwingung die Aufnahme gemacht wurde. So ist die Strömungsrichtung über die Dauer von drei Bildern (eine Periode) von der Sonotrode weg und eine Periode lang auf die Sonotrode zu gerichtet. Beim Betrachten der linken 


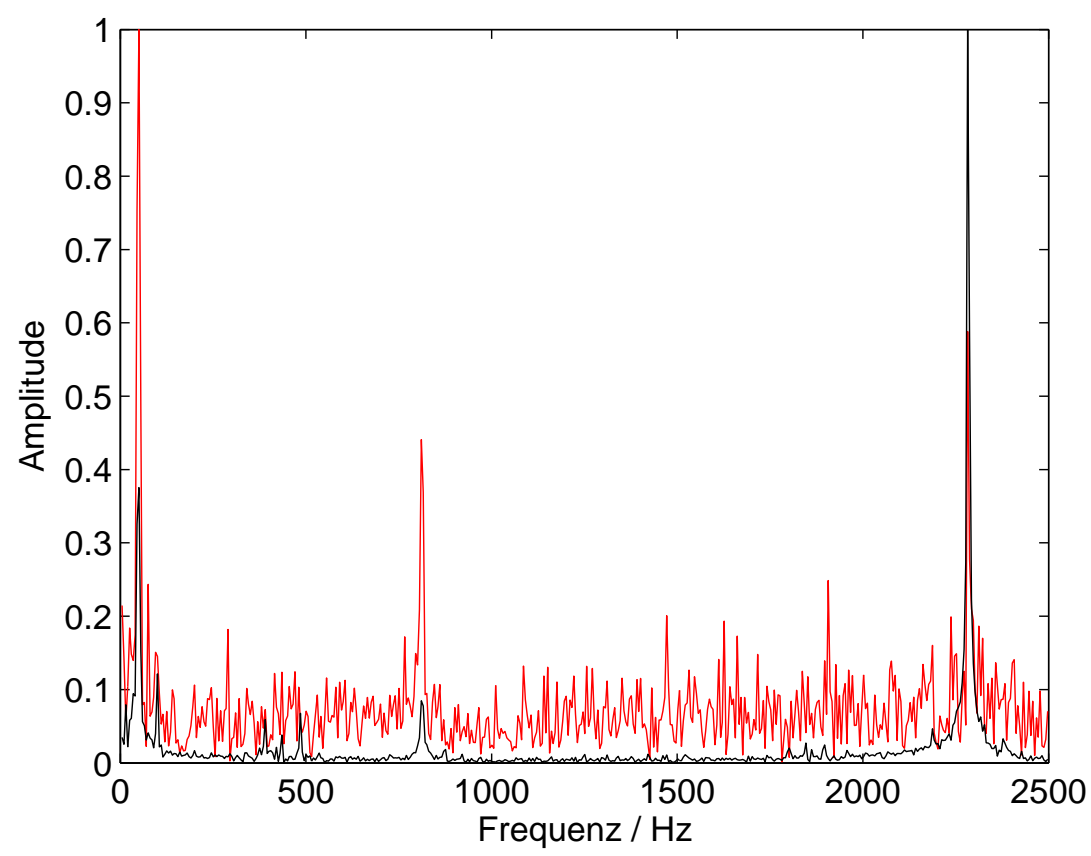

Abbildung 4.52.: Fourier Transformation der Geschwindigkeitsamplitude (rot) und der Anzahl der schwarzen Pixel (schwarz) bei $20^{\circ} \mathrm{C}, 5 \%$ Gasgehalt und $400 \mathrm{~V}$.

Spalte fällt auf, dass die große an der Sonotrode anhaftende Blase ebenfalls mit Periode zwei schwingt und die Strömung unterhalb dieser Schwingung größtenteils folgt.

Eine zeitliche Abfolge von Bildern bei $550 \mathrm{~V}$ ist in Abbildung 4.55 gezeigt. Die Anordnung der Bilder ist die gleiche wie in der vorherigen Abbildung. Genau wie oben ist hier der Wechsel der Strömungsrichtung zu erkennen. Zusätzlich ist im rechten Bildbereich jedes Einzelbildes eine Blasenwolke zu erkennen, die ihrerseits eine Strömung induziert. Vor allem in der rechten Spalte oben ist in den ersten vier Bildern (der Spalte) das Aufschwingen und Kollabieren anhand der Strömungsrichtung erkennbar. In diesem Fall laufen weniger Blasen auf die Sonotrode zu. Die Bewegung der Blasen auf die Sonotrode zu und die Strömung von der Sonotrode weg scheinen sich bei der höheren Spannung in etwa die Waage zu halten, so dass die Blasen auf einer Höhe bleiben. 


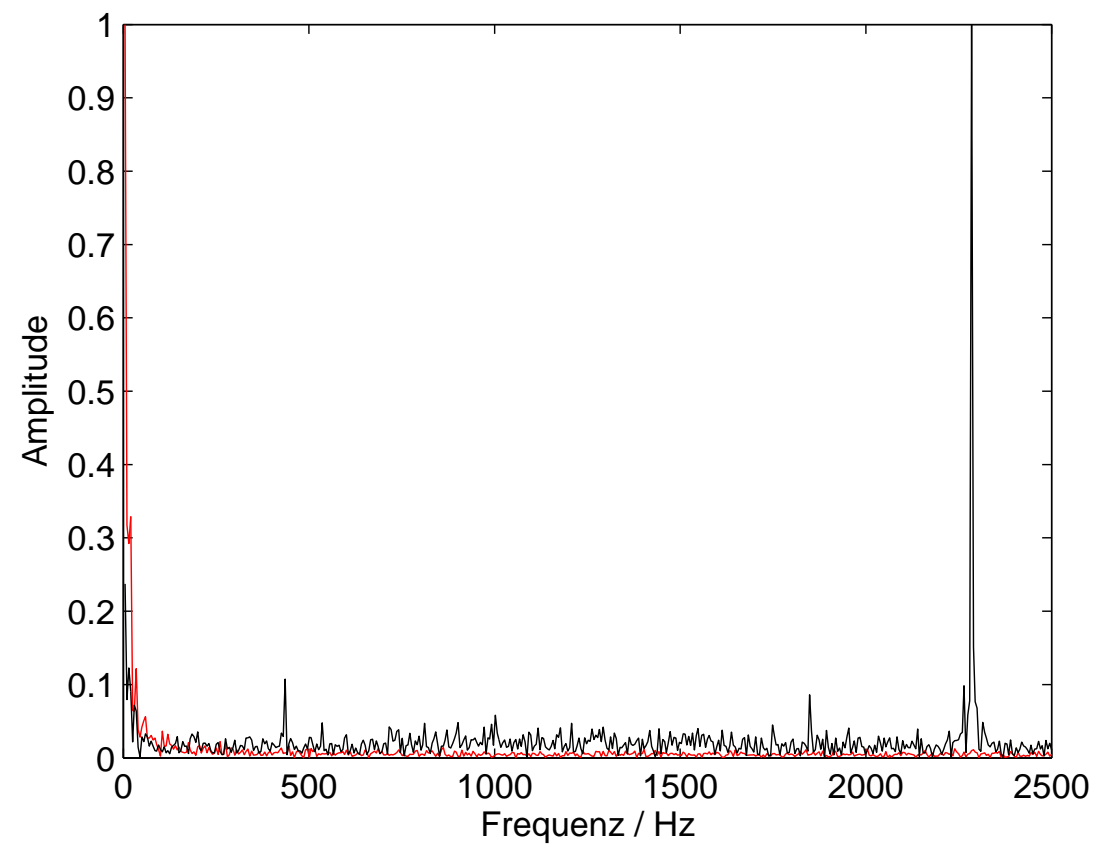

Abbildung 4.53.: Fourier Transformation der Geschwindigkeitsamplitude (rot) und der Anzahl der schwarzen Pixel (schwarz) bei $20^{\circ} \mathrm{C}, 90 \%$ Gasgehalt und $600 \mathrm{~V}$.

\subsubsection{Analyse von Blasenschwingung und Strömung}

Da die Aufnahmerate ein nicht ganzzahliges Vielfaches der treibenden Ultraschallfrequenz ist (ungefähr 2.89), erfolgt die Aufnahme eines Bildes in jeder Anregungsperiode immer etwas zeitversetzt. Dadurch lässt sich jedes Bild einer Phase der Periode zuordnen. In den Abbildungen 4.56 bis 4.58 ist in Teilabbildung (a) jeweils die mittlere vertikale Geschwindigkeitskomponente gegen zwei Perioden des Schallfeldes in rot aufgetragen. In schwarz ist in den selben Abbildungen jeweils die normierte Anzahl der schwarzen Pixel aufgetragen (siehe Kapitel 4.1.2). Diese Werte wurden aufgrund der besseren Darstellbarkeit um 0.6 nach oben verschoben eingezeichnet.

In Abbildung 4.56(a) ist anhand der schwarzen Punkte eine klare Blasenschwingung zu erkennen. Die Werte streuen im Maximum und während des Kollaps stark, im Aufschwingen weniger. Dies könnte mit der schwankenden Größenverteilung der Blasen zusammenhängen. Je nach Größe kollabieren die Blasen zu einer anderen Phase der Schallschwingung. Das Aufschwingen erfolgt aber relativ gleichphasig. 


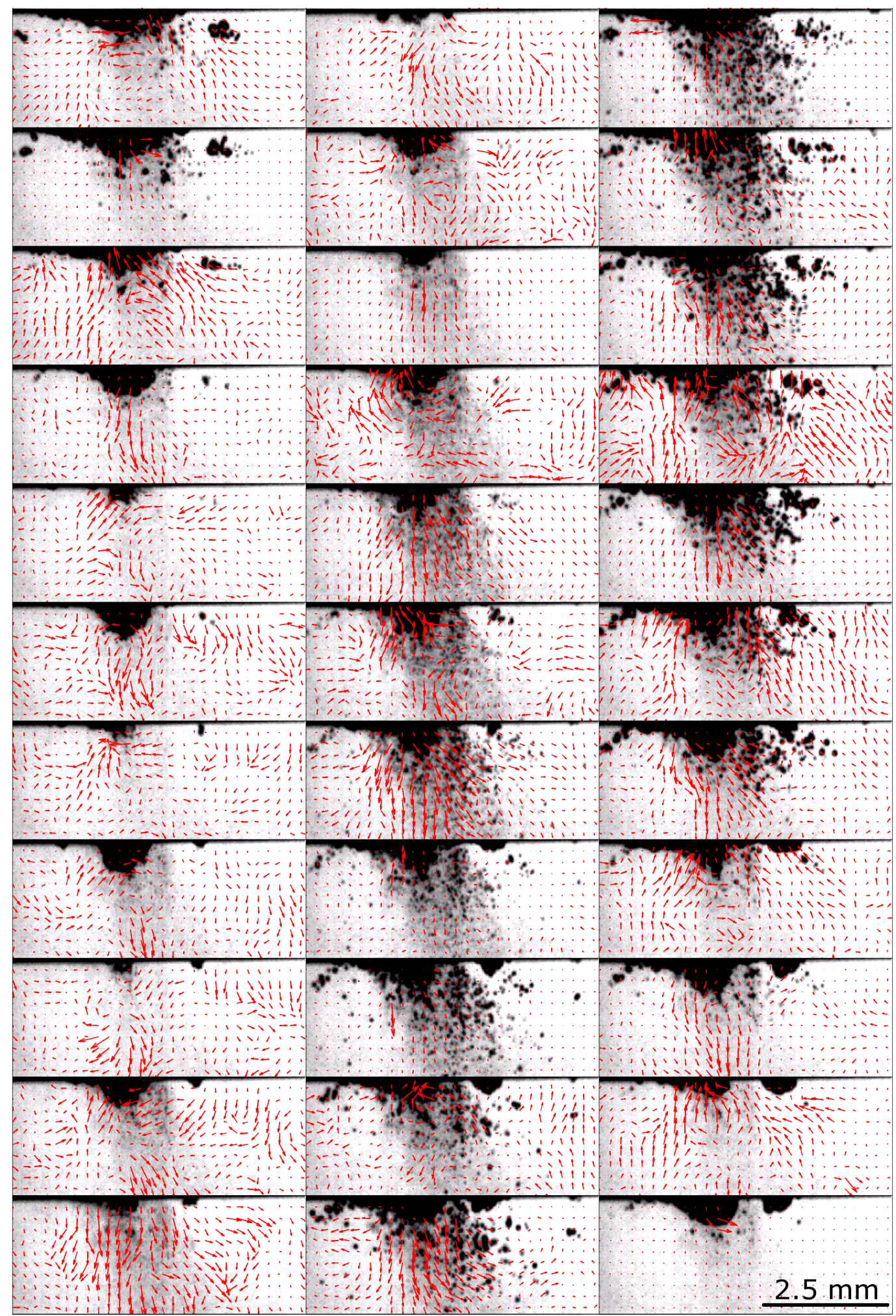

Abbildung 4.54.: Zeitlicher Verlauf der Kavitation und des Strömungsfeldes direkt an der Sonotrode bei 260 V. Der Film wurde mit 50000 Bps aufgenommen, die Belichtungszeit betrug etwa $1 \mu \mathrm{s}$. Die Zeit verläuft zeilenweise von links oben nach rechts unten. Die Bewegung der Blasen auf die Sonotrode zu ist gut zu erkennen. 


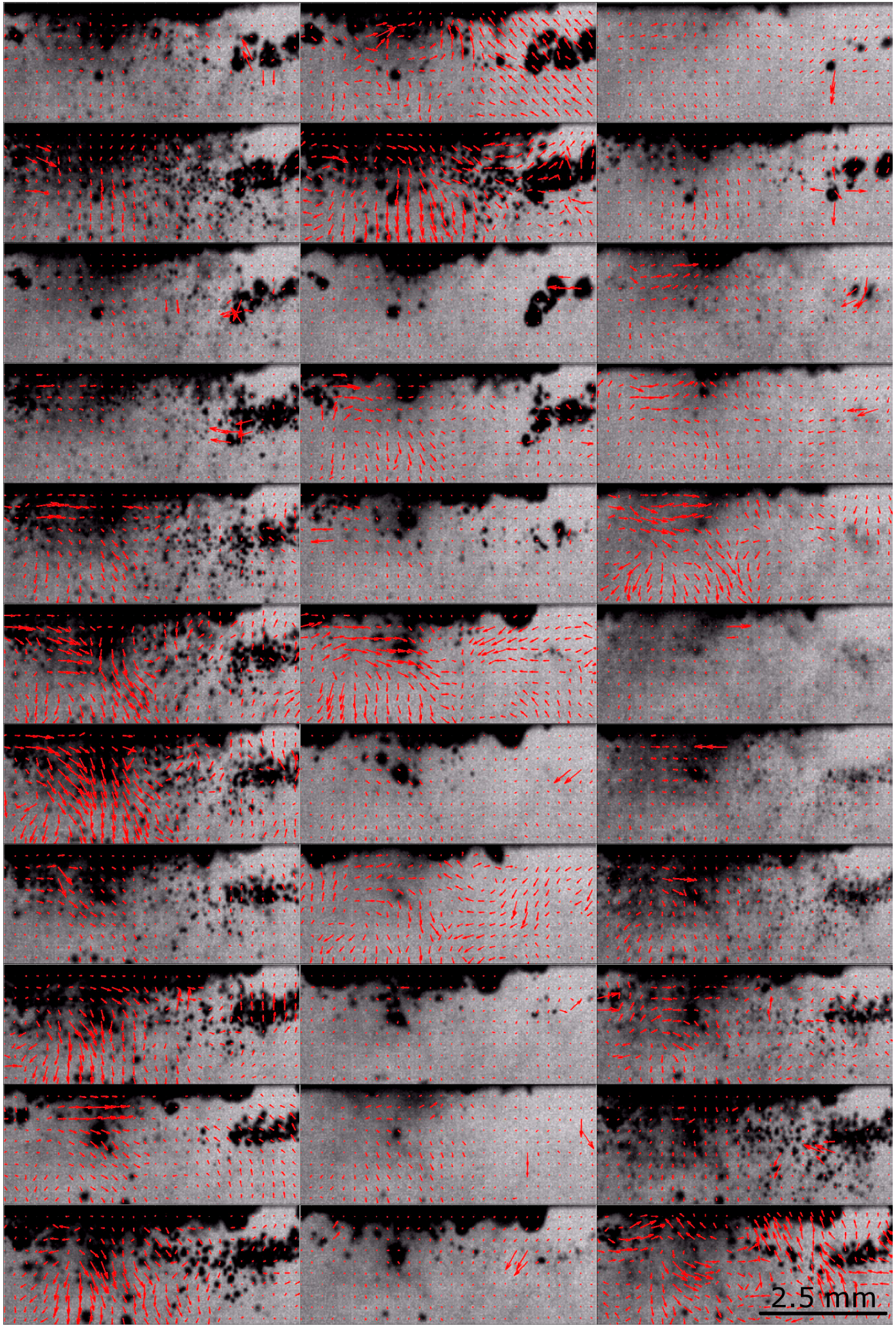

Abbildung 4.55.: Zeitlicher Verlauf der Kavitation und des Strömungsfeldes direkt an der Sonotrode bei 550 V. Der Film wurde mit 50000 Bps aufgenommen, die Belichtungszeit betrug etwa $1 \mu \mathrm{s}$. Die Zeit verläuft zeilenweise von links oben nach rechts unten. 
Die Auftragung der mittleren vertikalen Geschwindigkeitskomponente zeigt ebenfalls einen schwankenden, stark gestreuten Verlauf. Die Maximalwerte (von der Sonotrode weg) treten im Maximum des Blasenvolumens oder kurz danach auf. Ist das Blasenvolumen minimal wird die Geschwindigkeit minimal (auch negativ). In dieser Phase strömt die Flüssigkeit also auf die Sonotrode zu. In der unteren Abbildung 4.56(b) sind die jeweiligen Fouriertransformierten des zeitlichen Verlaufs der schwarzen Pixel und der mittleren vertikalen Geschwindigkeitskomponente dargestellt. Es sind eine deutliche Spitze bei der treibenden Frequenz von $17.381 \mathrm{kHz}$ und ein verbreiterter Bereich bei der halben Frequenz um $8150 \mathrm{~Hz} z u$ erkennen. Dies könnte durch die an der Sonotrode anhaftende Blase verursacht werden, die mit halber Frequenz schwingt.

Ein zweites Beispiel bei $550 \mathrm{~V}$ zeigt Abbildung 4.57. Genau wie in der vorherigen Abbildung sind die Messwerte der schwarzen Pixel stark gestreut. Im Maximum und Kollaps wiederum stärker als im Aufschwingen. Die mittlere vertikale Geschwindigkeitskomponente zeigt ebenfalls einen ähnlichen Verlauf wie in der vorherigen Abbildung. Die maximalen Geschwindigkeiten von der Sonotrode weg treten kurz vor oder im maximal aufgeschwungenen Zustand auf. Die minimalen Geschwindigkeiten (negativ, auf die Sonotrode zu) treten hier kurz vor und während des minimalen Blasenvolumens auf. Kurz nachdem das Blasenvolumen minimal war, streuen die Geschwindigkeitswerte kaum und sind nahe bei Null. Auch die Fouriertransformierte zeigt einen fast identischen Verlauf zum vorherigen Beispiel. Das Maximum der Amplitude bei der treibenden Frequenz von $17.381 \mathrm{kHz}$ ist deutlich ausgeprägt. Bei der halben Frequenz ist wieder der verbreiterte Bereich zu erkennen.

Bei niedrigerer Spannung von $260 \mathrm{~V}$ wurden ebenfalls die Zahl der schwarzen Pixel und die mittlere vertikale Geschwindigkeitskomponente ausgewertet. Die Ergebnisse sind in Abbildung 4.58 gezeigt. Hier streuen die Werte sehr viel weniger. Allerdings ist die Streuung der Werte der schwarzen Pixel wieder im und kurz nach dem Maximum am stärksten ausgeprägt. Es ist zu erkennen, dass die Blasenschwingung eine leichte Periode-zwei-Ausprägung hat. Dieser Effekt ist bei der Geschwindigkeitskomponente noch besser zu erkennen. Wie in den vorherigen Abbildungen treten die maximalen Geschwindigkeiten von der Sonotrode weg kurz vor und im Maximum der Zahl der schwarzen Pixel auf. Die minimalen Geschwindigkeiten, auf die Sonotrode zu, sind kurz vor dem Minimum des Blasenvolumens zu sehen. Der Periode-zwei-Effekt ist in der Auftragung der Fouriertransformierten zu sehen. Die treibende Frequenz erzeugt eine klare Spitze bei $17.381 \mathrm{kHz}$. Bei etwa der halben Frequenz ist hier bei $8717 \mathrm{~Hz}$ eine weitere, schmale und gut ausgeprägte Spitze zu sehen. In Abbildung 4.54 ist das Periode-zwei-Verhalten der Schwingung zu erkennen. Die Blase direkt an der Sonotrode schwingt nur jede zweite Periode stark auf. 


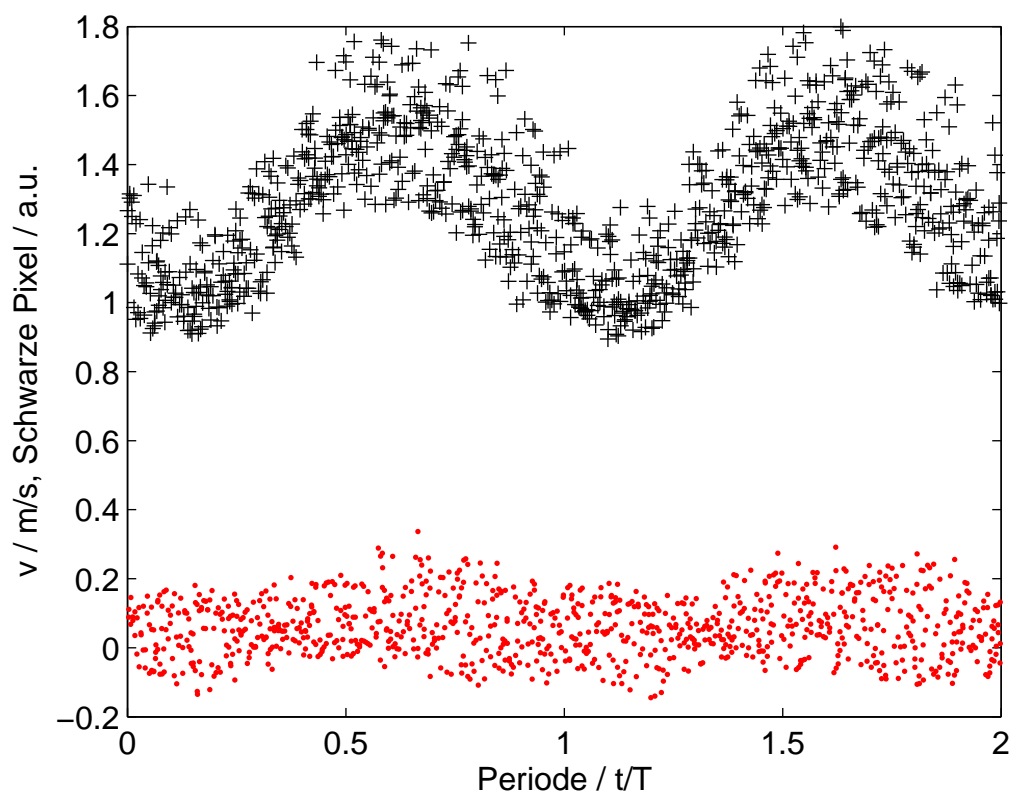

(a) Mittlere Vertikalkomponente der Geschwindigkeit (rot) und normierte Zahl der schwarzen Bildpunkte (schwarz) aufgetragen über der Periode. Zur besseren Darstellbarkeit wurden die Werte der schwarzen Bildpunkte um 0.6 nach oben verschoben.

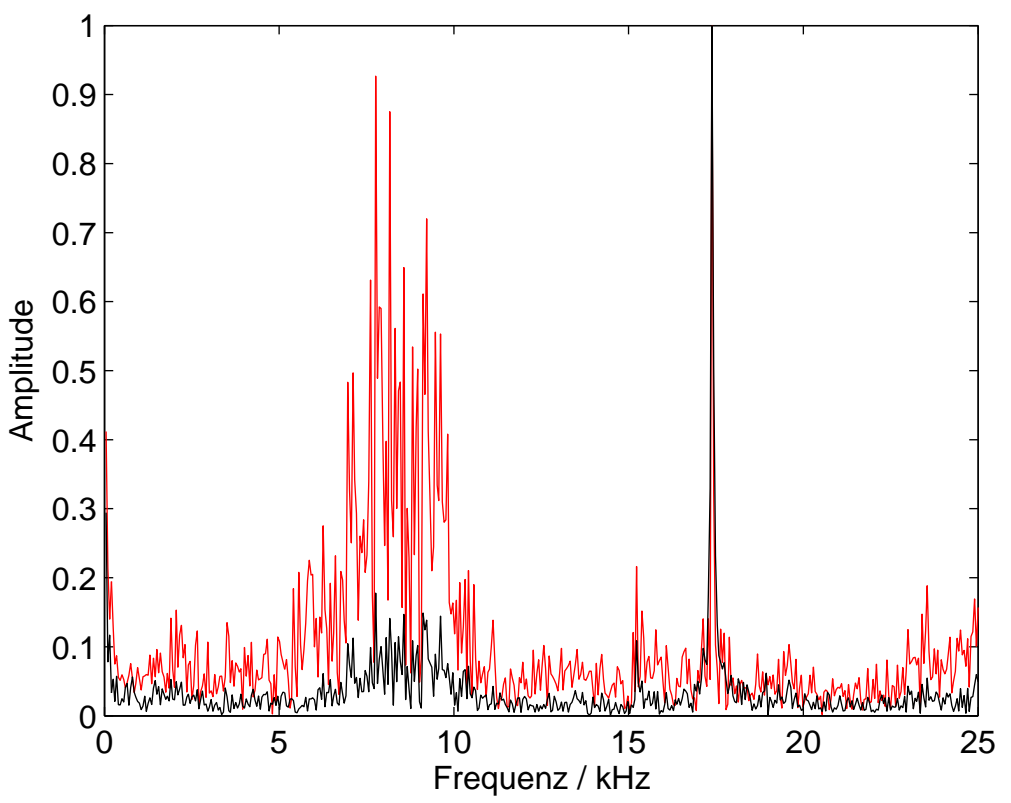

(b) Fourieranalyse des zeitlichen Verlaufs der mittleren Vertikalkomponente der Geschwindigkeit (rot) und der Anzahl der schwarzen Pixel (schwarz). Die Fundamentale der treibenden Frequenz bei $17.381 \mathrm{kHz}$ ist gut zu erkennen.

Abbildung 4.56.: Aufnahmen bei $17.381 \mathrm{kHz}$ und $550 \mathrm{~V}$. 


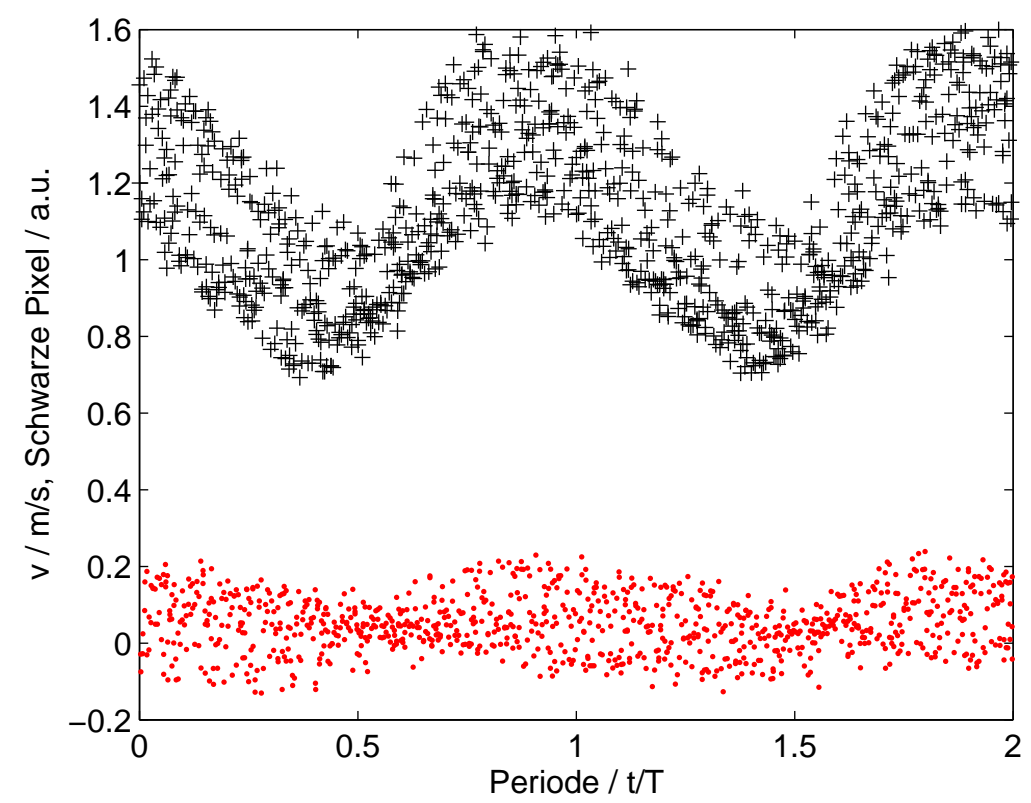

(a) Mittlere Vertikalkomponente der Geschwindigkeit (rot) und normierte Zahl der schwarzen Bildpunkte (schwarz) aufgetragen über der Periode. Zur besseren Darstellbarkeit wurden die Werte der schwarzen Bildpunkte um 0.6 nach oben verschoben.

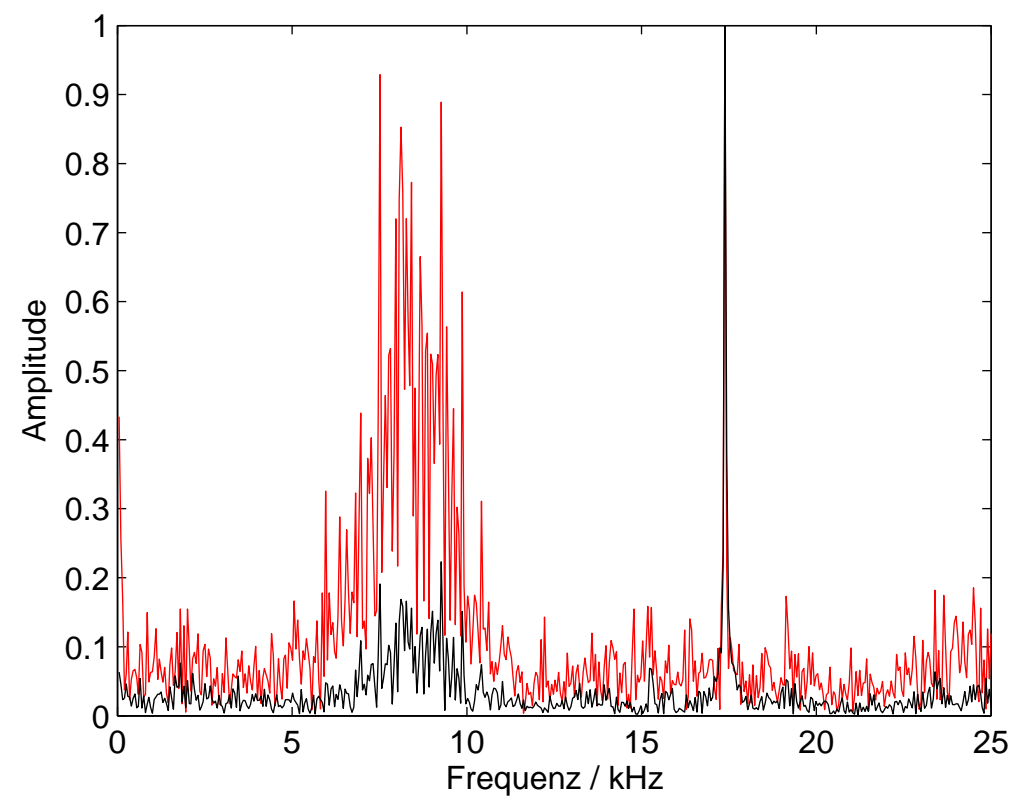

(b) Fourieranalyse des zeitlichen Verlaufs der mittleren Vertikalkomponente der Geschwindigkeit (rot) und der Anzahl der schwarzen Pixel (schwarz). Die Fundamentale der treibenden Frequenz bei $17.381 \mathrm{kHz}$ ist gut zu erkennen.

Abbildung 4.57.: Aufnahmen bei $17.381 \mathrm{kHz}$ und $550 \mathrm{~V}$. 


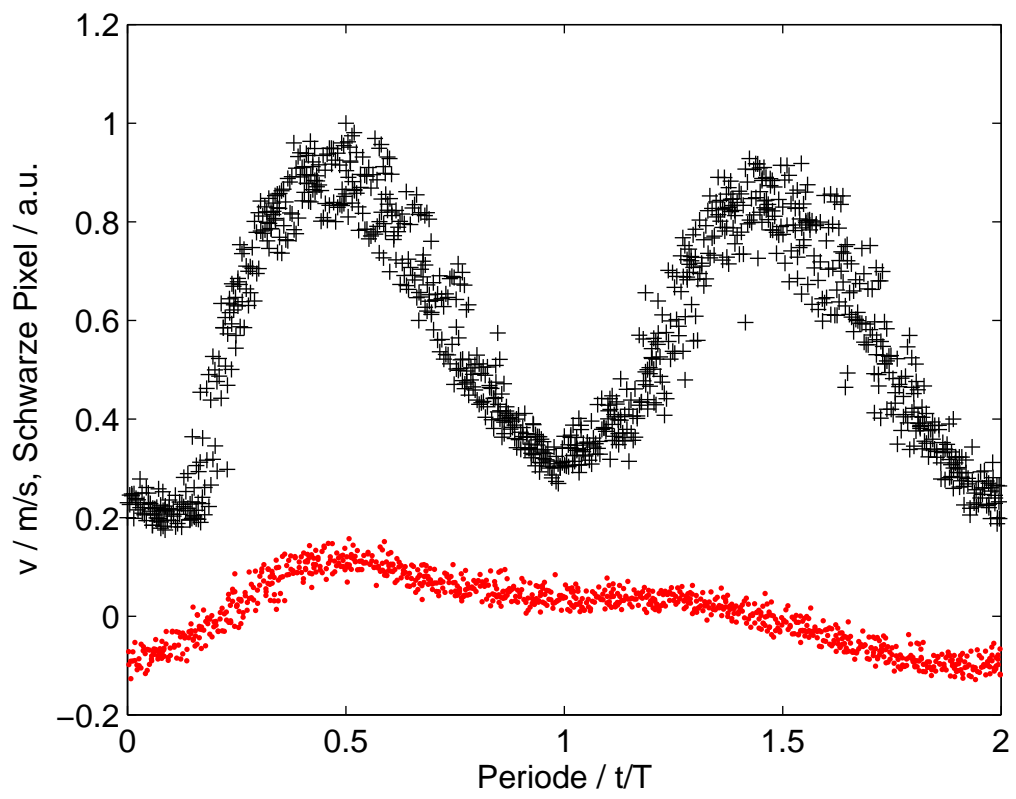

(a) Mittlere Vertikalkomponente der Geschwindigkeit (rot) und normierte Zahl der schwarzen Bildpunkte (schwarz) aufgetragen über der Periode bei $17.381 \mathrm{kHz}$ und $260 \mathrm{~V}$. Zur besseren Darstellbarkeit wurden die Werte der schwarzen Bildpunkte um 0.6 nach oben verschoben.

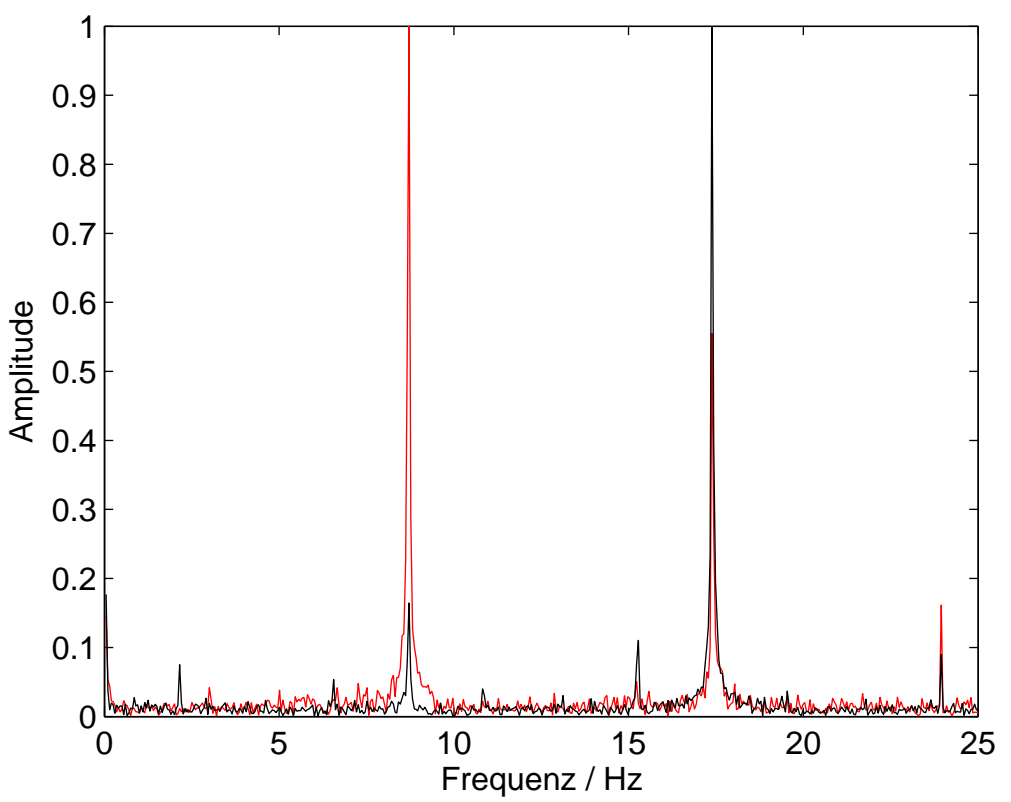

(b) Fourieranalyse des zeitlichen Verlaufs der mittleren Vertikalkomponente der Geschwindigkeit (rot) und der Anzahl der schwarzen Pixel (schwarz). Die Fundamentale der treibenden Frequenz bei $17.381 \mathrm{kHz}$ ist gut zu erkennen.

Abbildung 4.58.: Aufnahmen bei $17.381 \mathrm{kHz}$ und $260 \mathrm{~V}$. 


\section{Kapitel 5 \\ FEM-Simulation von Schallfeld und Strömung}

Mit dem kommerziellen Software-Paket Comsol 4.3a [44] wurde das Schallfeld in der Küvette für verschiedene Auslenkungen der Sonotrode (der Grenzfläche zwischen Sonotrode und Wasser in z-Richtung) mit der Finite-Elemente-Methode (FEM) dreidimensional simuliert. Hierzu wurde das Acoustics-Modul mit dem Unterpunkt Acoustic-Solid Interaction benutzt. Die Simulation wurde in diesem Fall auf die Küvette inklusive der Wände, Verschlussschrauben und Sonotrodenspitze beschränkt. Bei den Eigenschaften der Materialien wurden die in COMSOL vorhandenen benutzt. An der vorderen Fläche der Sonotrodenspitze wurde eine Auslenkung mit einer Frequenz von $17.281 \mathrm{kHz}$ definiert. Das verwendete Gitter besteht aus 267791 Elementen mit einer maximalen Größe von $2.562 \times 10^{-3} \mathrm{~m}$ und einer minimalen Größe von $1.1 \times 10^{-4} \mathrm{~m}$. Die Rechenzeit betrug etwa sechs Minuten. In Abbildung 5.1 ist zu erkennen, dass das Schallfeld eine stehende Welle ausbildet, die unsymmetrisch ist. Die Asymmetrie liegt an dem Aufbau der Küvette: In Betrachtungsrichtung sind die linke, die obere und die untere Wand aus Stahl, die rechte Wand aus Plexiglas. Daher sind die Reflexionseigenschaften verschieden. Die hier abgebildete Blickrichtung entspricht der in allen Aufnahmen dieser Arbeit verwendeten. Die dargestellte Wechseldruckamplitude reicht bis 10.2 bar. Das Druckmaximum befindet sich direkt an der Sonotrodenspitze. Druckminima befinden sich in der rechten oberen Ecke und auf einem Streifen von der linken Stahlwand bis zur rechten Kante der Bodenverschlussschraube. Wird der Druckverlauf von der Sonotrodenspitze bis zum Boden der Küvette in Abhängigkeit von der Auslenkung aufgetragen (siehe Abbildung 5.2), ist zu erkennen, dass der Druck an der Sonotrodenspitze linear mit der Auslenkung zunimmt. Innerhalb von $5 \mathrm{~mm}$ Entfernung nimmt der Schalldruck um etwa $60 \%$ ab. In ungefähr $27 \mathrm{~mm}$ Entfernung befindet sich ein Druckknoten. Messungen des Schallfeldes konnten aufgrund der geschlossenen Bauweise bei der verwendeten Küvette nicht durchgeführt und zum Vergleich herangezogen werden. Eine 
$\boldsymbol{\Delta} 10.212$

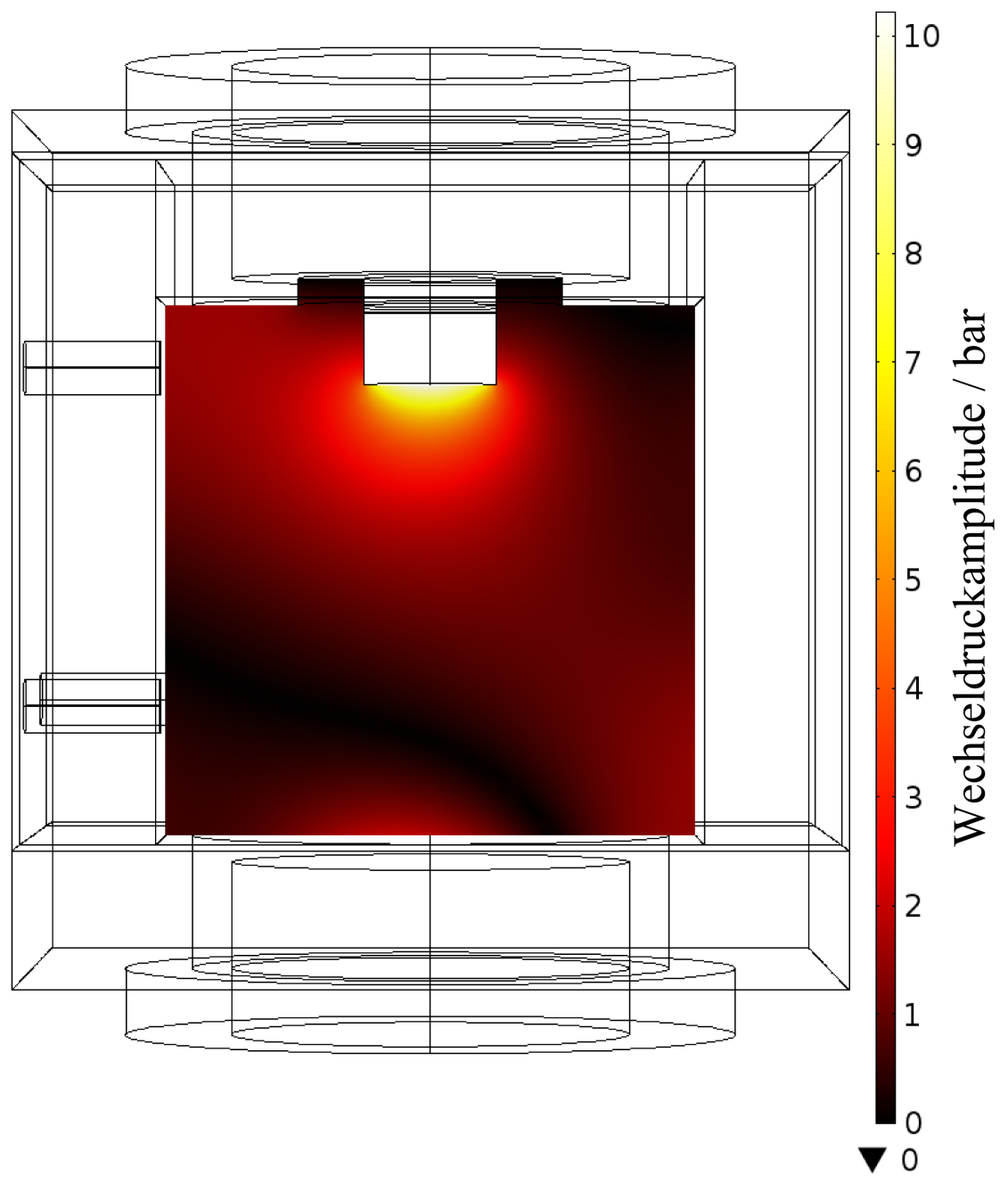

Abbildung 5.1.: 3D-Simulation des Schallfeldes in der Küvette bei $17.281 \mathrm{kHz}$. Simulation durchgeführt mit dem Acoustics-Modul Modul von Comsol mit dem Unterpunkt Acoustic-Solid Interaction. Vorgegebene Auslenkungsamplitude der Sonotrode $20 \mu \mathrm{m}$. Auf der linken Seite befindet sich die Stahlwand mit den Bohrungen für die Anschlüsse, oben und unten die Stahlwände mit den Verschlussschrauben. Die anderen Wände bestehen aus Plexiglas (PMMA). Der Durchmesser der Sonotrodenstirnfläche beträgt $1 \mathrm{~cm}$

Schallwechseldruckamplitude von 10 bar ist bei der gegebenen Auslenkung, die der gemessenen entspricht, realistisch. Mit Kavitation wird sich der Druck durch das Aufreißen der Flüssigkeit und die dadurch resultierende Dämpfung stark reduzieren. 


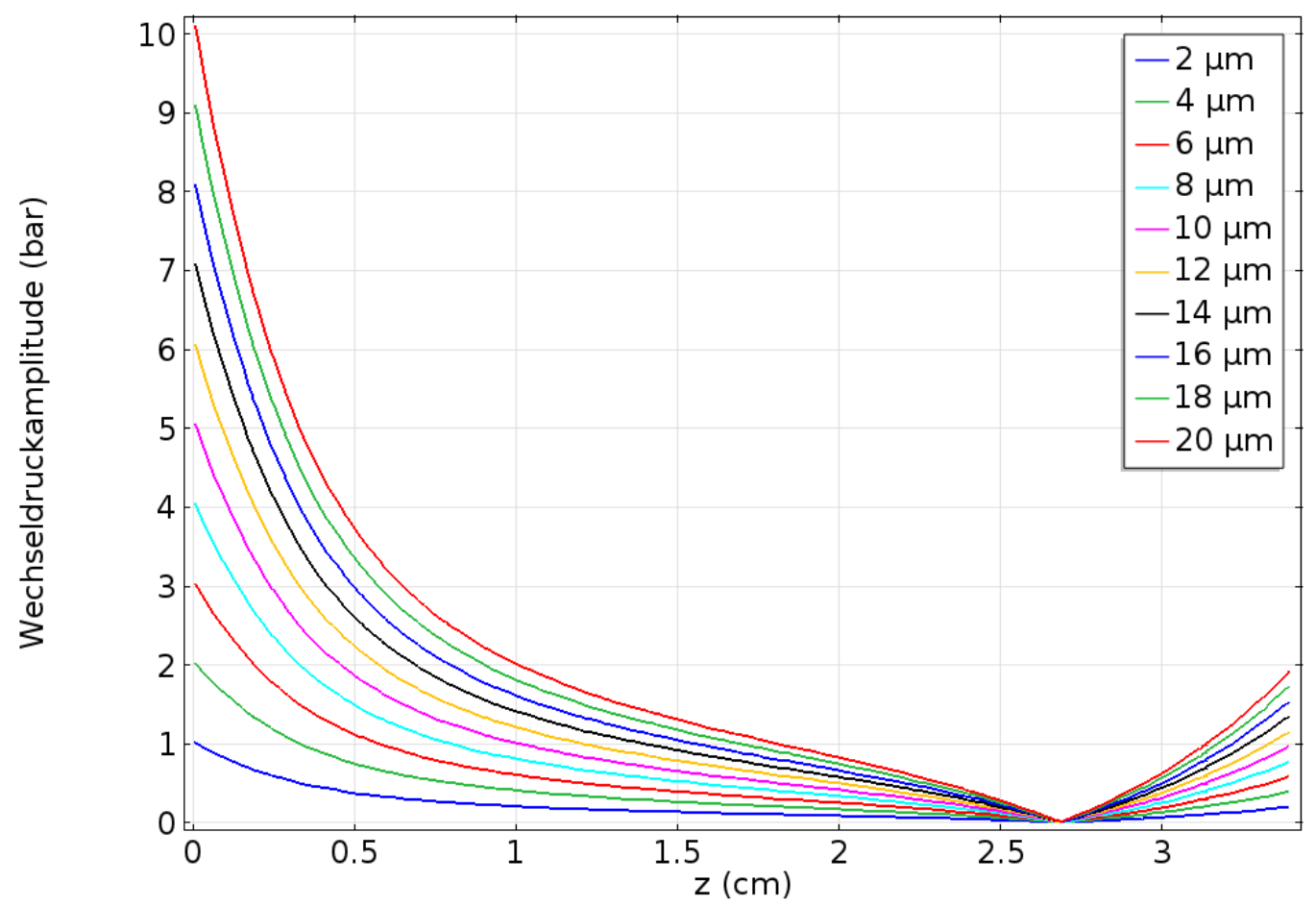

Abbildung 5.2.: Simulation des Schallfeldes in der Küvette bei $17.281 \mathrm{kHz}$. Simulation durchgeführt mit dem Acoustics-Modul von ComSOL mit dem Unterpunkt Acoustic-Solid Interaction. Aufgetragen ist der Druckverlauf in der Mitte der Küvette von der Sonotrodenspitze bis zum Boden, mit der Sonotrodenauslenkung als Parameter.

Es wurde außerdem versucht, eine zweidimensionale Simulation der akustisch induzierten Strömung durchzuführen. Hierzu wurde zusätzlich zur Acoustic-Solid Interaction der Unterpunkt Laminar Flow verwendet. Das Gitter besteht aus 26612 Elementen mit einer Minimalgröße von $1.6 \times 10^{-6} \mathrm{~m}$ und einer Maximalgröße von $8.0 \times 10^{-4} \mathrm{~m}$. Ein erstes Ergebnis ist in Abbildung 5.3 gezeigt. In der Simulation gelang es allerdings nicht eine stabile Lösung zu erhalten. Die Strömung wird während der instationären Rechnung immer schneller und instabil, anschließend bricht die Simulation ab. Eventuell könnte durch Verwenden anderer Lösungsalgorithmen oder den Einbau einer Dämpfung eine stabile Lösung zu erhalten sein, was aber nicht weiter untersucht wurde. Für die abgebildete Lösung wurde ein Zeitschritt kurz vor Beginn der sichtbaren Instabilität und mit realistischen Geschwindigkeiten verwendet. An den Ecken der Sonotrodenspitze und den Kanten der Gummidichtung sind Verwirbelungen zu erkennen. Die Strömung von der Sonotrode weg bildet sich erst nach etwa $1 \mathrm{~cm}$ voll aus. Oberhalb davon strömt die Flüssigkeit von den Ecken der Sonotrodenspitze schräg nach unten. 


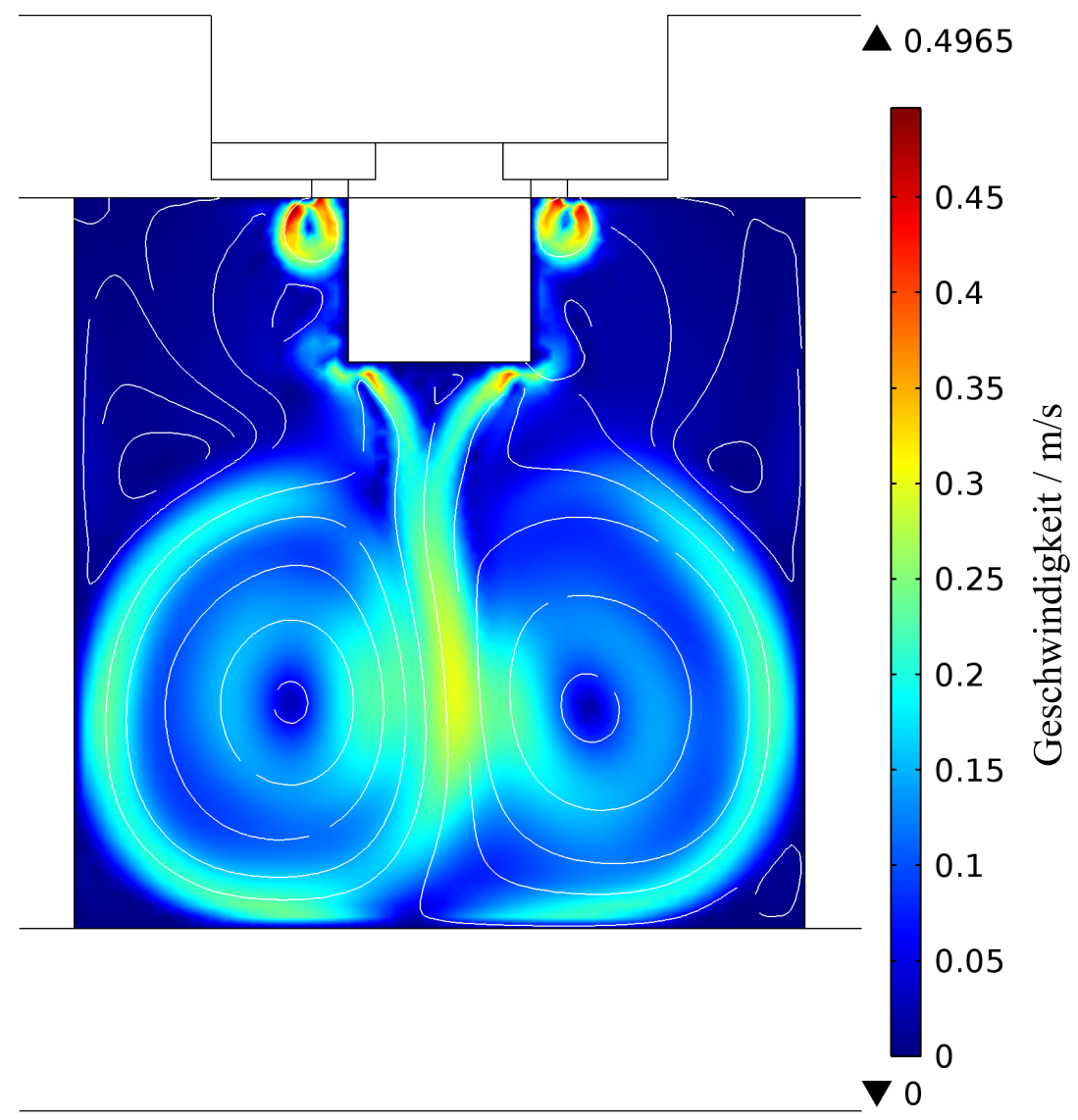

Abbildung 5.3.: Simulation des Strömungsfeldes in der Küvette bei $17.281 \mathrm{kHz}$ und $5 \mu \mathrm{m}$ Auslenkung. Simulation durchgeführt mit dem Acoustics-Modul von Comsol mit den Unterpunkten Acoustic-Solid Interaction und Laminar Flow. 


\section{Kapitel 6 Diskussion der Ergebnisse mit und ohne Kavitation}

\subsection{Strömungsfelder mit und ohne Kavitation}

Das Strömungsfeld unter der Sonotrode ändert sich mit Einsetzen der Kavitation massiv [93]. So nimmt die maximale Strömungsgeschwindigkeit etwa um zwei Größenordnungen zu. Außerdem wird die Strömung wesentlich kleinräumiger und der Bereich hoher Strömungsgeschwindigkeit (im Vergleich zur Strömungsgeschwindigkeit außerhalb) wird sehr viel schmaler. Der Bereich der schnellen Strömung ist scharf begrenzt. Erst mit zunehmender Spannung findet wieder eine Verbreiterung statt. Dieser Effekt des schmaler werdenden Strömungsfeldes ist besonders gut in den gemittelten Strömungsfeldern mit Kavitation zwischen 500 und $600 \mathrm{~V}$ zu erkennen (siehe Abbildung 4.16). Hier ist auch die im oberen Bildbereich abgedruckte maximal vorkommende Geschwindigkeit zeitlich gemittelt, deren Richtung mit der Zeit fluktuiert. Dies führt dazu, dass der vektorielle Mittelwert wesentlich niedriger ist als der vorkommende Maximalwert. In den Geschwindigkeitsfeldern mit Kavitation treten die Maximalgeschwindigkeiten sehr lokal auf. Dies liegt daran, dass das Strömungsfeld sich aus zwei Komponenten zusammensetzt. Zum einen aus den akustischen Strömungen, die durch Dämpfung des Schalls verursacht werden, zum anderen aus durch Einzelblasen oder Blasencluster verursachte Strömungen, sogenannte Mikroströmungen [17]. So erzeugen schnell wandernde Blasen ein Strömungsfeld mit Verwirbelungen, die sich der akustischen gleichgerichteten Strömung überlagern [104,105]. Auch der Blasenkollaps und Oberflächenschwingungen der Blasen erzeugen Mikroströmungen. Diese Mikroströmungen können lokal sehr hohe Geschwindigkeiten hervorrufen. Die Strömungen, die beim Blasenkollaps erzeugt werden, werden allerdings in diesen Versuchen nicht aufgelöst, da über drei Schwingungsperioden gemittelt wird. Ansonsten wären noch sehr viel höhere Geschwindigkeiten zu erwarten gewesen [90]. Eine Schwierigkeit besteht bei den Aufnahmen mit Kavitation darin, 
dass die Kavitationsblasenwolke nicht immer in die gleiche Richtung strömt, sondern hin und her springt. Die Kavitationsblasenwolke haftet mal vorne und mal hinten an der Sonotrode an (Variation der Tiefendimension), so dass das Hauptströmungsfeld, das, wie in den Aufnahmen gezeigt, mit der Blasenwolke zusammenfällt, nicht immer mittig geschnitten wird. Bei einem Schnitt ganz am Rand fiel dies sofort auf und die Aufnahme wurde wiederholt. Wurde das Strömungsfeld aber nur knapp neben der Mitte geschnitten, so dass nur die Maximalgeschwindigkeiten niedriger, aber die Ausdehnung ähnlich war, fiel dies erst beim Auswerten auf und die Messung konnte nicht wiederholt werden. Eventuell ist dies ein Grund für die Fluktuation der maximalen Geschwindigkeiten mit zunehmender Spannung in einigen Aufnahmen.

\subsection{Zusammenhang zwischen Kavitations- und Strömungsfeld}

Die abgebildeten Aufnahmen in Kapitel 4.5 zeigen eine starke räumliche Übereinstimmung zwischen Kavitationsblasenfeld und Bereichen hoher Strömungsgeschwindigkeit. Dies liegt an einer Zunahme der Schalldämpfung und Erzeugung von sehr schnellen Mikroströmungen durch die vorhandenen Blasen. Die starke Übereinstimmung der Grenzen beider Gebiete war nicht zwingend zu erwarten. Durch die Erhöhung der Dämpfung beeinflussen sich die Blasen und das Schallfeld wechselseitig. Eine hohe Schalldruckamplitude trägt zum Entstehen vieler Kavitationsblasen bei, die stark schwingen und dadurch das Schallfeld stark dämpfen. Dies wiederum führt zu einer hohen Strömungsgeschwindigkeit, die vor allem kleine Blasen mitreißt. Diese Blasen wirken nun eventuell in Gebieten mit niedrigerem Schalldruck, der unterhalb der Kavitationsschwelle liegt, als Kavitationskeime, so dass dort trotzdem Kavitation einsetzt und ihrerseits hohe Geschwindigkeiten durch Mikroströmungen erzeugt. Des Weiteren sinkt in dem Gebiet, in dem die Blasen ursprünglich entstanden sind, durch den Wegtransport der Blasen die Dämpfung und der Schalldruck dahinter nimmt zu, was wiederum höhere Strömungsgeschwindigkeiten und das Entstehen von Kavitationsblasen zur Folge hat. Diese Rückkopplungseffekte können sowohl zu räumlichen als auch zu zeitlichen Fluktuationen des Blasenfeldes und damit auch des Strömungsfeldes führen. Auch lassen sich eventuell subharmonische Frequenzen in Schalldruckmessungen durch diese Effekte erklären. 


\subsection{Zunahme der kinetischen Energiedichte mit der Spannung}

Die Zunahme der kinetischen Energiedichte mit der Spannung folgt in den durchgeführten Versuchen verschiedenen Abhängigkeiten. So wurden Proportionalitäten zwischen $U_{\mathrm{el}}^{4}$ und $U_{\mathrm{el}}^{10}$ festgestellt. In einigen Fällen traten auch exponentiell ansteigende Verläufe auf. Laut den theoretischen Betrachtungen sollte die Zunahme der Geschwindigkeit im laminaren Strömungsfeld ohne Kavitation eine Proportionalität $\mathrm{zu} U_{\mathrm{el}}^{2}$ aufweisen (siehe Kapitel 2.1.2). Die kinetische Energiedichte sollte also proportional $\mathrm{zu} U_{\mathrm{el}}^{4}$ verlaufen. Dies ist allerdings in den durchgeführten Messungen hauptsächlich mit Kavitation der Fall. Bei den Ergebnissen ohne Kavitation zeigen die Messwerte meist einen zu $U_{\text {el }}^{6}$ proportionalen Verlauf. Der Grund für höhere gemessene Exponenten liegt vermutlich im Mittelungsverfahren über ein festes Auswertungsfenster. Bei Einkopplung des Schalls in das Wasser wird eine bestimmte kinetische Energie übertragen. Da in einer geschlossenen Küvette das Wasser an den Seiten zurück strömen muss, bilden sich Wirbel aus, die aufgrund der Energieerhaltung eine maximale Energie enthalten können. Ist die Geschwindigkeit innerhalb des Strömungsfeldes sehr klein, breitet sich das Strömungsfeld nicht bis ans Ende der Küvette aus. Da das Fenster, über das die kinetische Energiedichte gemittelt wurde, konstant gehalten wurde und teilweise größer ist als das Strömungsfeld, nimmt die kinetische Energiedichte mit der Größe der Schnittfläche des messbaren Strömungsbereichs im Beobachtungsfenster zu. Vergrößert sich die übertragene kinetische Energie, nimmt auch das Volumen des beschleunigten Wassers zu und die Wirbel werden größer. Diese Zunahme der Wirbelgröße und damit die Vergrößerung des Strömungsfeldes wurde bereits in Kapitel 4.3 beschrieben.

Ein Aspekt der Messungen lässt sich allerdings nicht über geometrische Effekte erklären. So ist der Anstieg der kinetischen Energiedichte bei zwei bar Überdruck (und teilweise auch bei Normaldruck) nach dem Überschreiten der Kavitationsschwelle besonders steil, um nach einiger Zeit ähnliche Energiedichten zu erreichen, die auch bei den Messungen ohne Überdruck bei Kavitation erreicht wurden. Es gibt also einen Aufholeffekt der kinetischen Energiedichte. Da das Wasser in der Küvette zwischen den einzelnen Messungen regelmäßig ausgetauscht wurde, ist auch davon auszugehen, dass es sich nicht um zeitliche (Gedächtnis-)Effekte handelt. Eine Erklärung wäre, dass die Anzahldichte der Kavitationsblasen ab einer bestimmten Druckdifferenz zwischen Umgebungsdruck und anregendem Druck durch entstehende Abschirmung des Schalls gesättigt ist und nicht weiter zunimmt, was zu einem Maximalwert vorhandener 
Kavitationsblasen und somit ab dieser Grenze zu einem Verlauf der erwarteten Proportionalität führen würde. Der Aufholeffekt könnte darin begründet liegen, dass nach Überschreiten der Kavitationsschwelle direkt an der Sonotrode Kavitationskeime erzeugt werden, die von der Strömung mitgetragen werden und Kavitation weiter entfernt von der Sonotrode möglich machen. Durch die Blasen wird wiederum die Dämpfung erhöht, was zu einer schnelleren Strömung führt. Auch die durch den Überdruck veränderte Kollapsdynamik könnte eine Rolle spielen.

Allerdings ist die Genauigkeit der Messungen insgesamt kritisch zu sehen. So war die Kavitation an der Sonotrode besonders bei niedrigen Gasgehalten und Temperaturen relativ instabil und wechselte häufig die Position an der Sonotrodenspitze und die Richtung der Strömung. Wenn die Position an der Sonotrodenspitze sich bei einer Messung reproduzierbar verlagerte, wurde die Lichtschnittebene leicht verschoben, um das Strömungsfeld möglichst vollständig abzubilden. Dies hatte allerdings zur Folge, dass das Strömungsfeld ohne Kavitation nicht unbedingt mittig getroffen wurde, was den Flächenzunahmeeffekt durch einfache geometrische Effekte verstärkt. Unter der Annahme, dass sich die Strömung zylinderförmig ausbildet, lässt sich leicht verdeutlichen, dass mit größer werdendem Radius die Zunahme der Fläche im asymmetrischen Fall größer ist als im symmetrischen Fall, also bei mittigem Schnitt des Zylinders (siehe Abbildung 6.1).
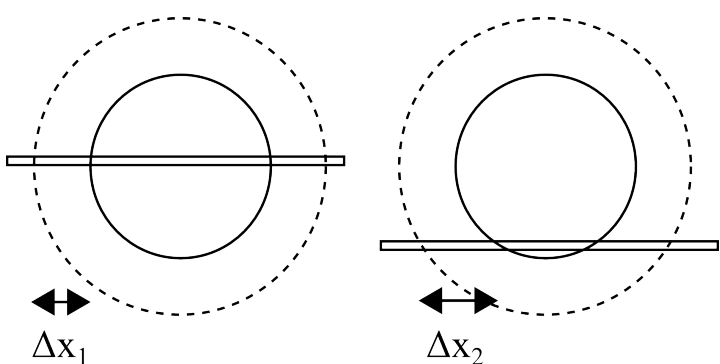

\begin{abstract}
Abbildung 6.1.: Geometrische Abhängigkeit der Größe des sichtbaren Strömungsbereiches in Abhängigkeit von der Lage der Schnittebene bei zylinderförmiger Strömung von oben betrachtet. Bei gleicher Vergrößerung des Strömungsbereiches ist die Zunahme der Fläche größer, wenn der Strömungsbereich am Rand geschnitten wird.
\end{abstract}

Zusammenfassend lässt sich sagen, dass die erwartete Zunahme der kinetischen Energie im (Gleich-)Strömungsfeld mit (Spannung) ${ }^{4}$ mindestens bestätigt wurde, wobei offenbar verschiedene Faktoren zu einem noch deutlich stärkeren Anstieg bei den Messwerten führen können. 


\subsection{Turbulenzmaße Korrelation und Reynolds- Zahl}

Der Übergang von akustisch getriebener Strömung ohne Kavitation zur Strömung mit Kavitation ähnelt im gezeigten Experiment stark dem Übergang zu Turbulenz [101]. Um dies quantitativ zu fassen, wurden Reynolds-Zahlen und Korrelationen des Strömungsfeldes bestimmt (siehe Kapitel 4.7.1 und 4.7.2).

Die Auswertung der Kreuzkorrelation der Strömungen ergab, bei geeigneter Wahl des Auswertungsfensters einen klaren Unterschied zwischen Strömungen mit und ohne Kavitation. Die Strömungen ohne Kavitation sind im Hauptströmungsfeld stark korreliert. Dies deutet auf eine rein laminare Strömung hin. Außerhalb der Hauptströmung tritt durch die sehr langsame Geschwindigkeit beim Auswerten mit der PIV-Software ein starkes Rauschen auf, was die Strömung unkorreliert erscheinen lässt. Mit Kavitation schwankt die Korrelation sehr stark zwischen korrelierter und unkorrelierter Strömung. Dies deutet auf einen ständigen Wechsel zwischen laminaren und turbulenten Phasen hin. Auch dieser Effekt könnte auf die Wechselwirkung zwischen Schallfeld, Blasenwolke und Strömungsfeld zurückzuführen sein. So ist in den aufgenommenen Filmen immer wieder ein Ablösen einer Blasenwolke von der Sonotrode zu erkennen, die anschließend durch die eventuell selbst erzeugte Strömung nach unten getragen wird.

Die erreichten Reynolds-Zahlen zeichnen ein ähnliches Bild. So weist die Strömung ohne Kavitation sehr geringe Reynolds-Zahlen bis maximal 100 auf. Dies spricht ebenfalls für eine rein laminare Strömung. Die Strömungen mit Kavitation lassen sich mit Reynolds-Zahlen bis etwa 3350 charakterisieren. Bei einem freien Jet geht die Strömung bei Reynolds-Zahlen über 3500 in eine turbulente Strömung über [102]. Allerdings gibt es einige Unterschiede zwischen der Strömung eines freien Jets und den untersuchten Strömungen im Kavitationsblasenfeld. So strömt beim freien Jet immer neue Flüssigkeit mit einer bestimmten Geschwindigkeit in das beobachtete Flüssigkeitsvolumen nach. Bei der kavitationsinduzierten Strömung wird die Strömung durch die sich ausbreitende Schallwelle an jedem Punkt weiter beschleunigt. Diese Beschleunigung ist aber durch die geringe Dämpfung bei der verwendeten Frequenz recht gering, was sich an der langsamen, laminaren Strömung ohne Kavitation ablesen lässt. Nach Überschreiten der Kavitationsschwelle treten weitere Effekte auf. So nimmt durch das Vorhandensein der Blasen die Dämpfung zu, was eine schnellere Strömung zur Folge hat. Die Strömung nimmt durch die jetzt in der Nähe des Schallwandlers wesentlich höhere Strömungsgeschwindigkeit, die durch den hohen Gradienten des Schalldrucks zustande kommt, eine jet-artige Form an. Außerdem verursachen 
die Blasen selber durch ihre Bewegung, Oszillation und Oberflächenmoden eigene, sehr kleinräumige Strömungen, die unabhängig von der Hauptströmung sind und ihr auch entgegengesetzt gerichtet sein können [70,106]. Durch die Blasen werden also lokale turbulente Strömungen verursacht, die einen großen Einfluss auf die Korrelationen haben, die aber nicht durch die Reynolds-Zahl, die aus der mittleren Vertikalkomponente der Strömungsgeschwindigkeit berechnet wurde, erfasst werden.

\subsection{Vergleich der Energien}

Der Vergleich der kinetischen Energie mit der abgestrahlten Schallenergie (Kolbenstrahlerenergie) und der in aufgeschwungenen Blasen gespeicherten Volumenenergie zeigt, dass die Energien relativ gut dem erwarteten Verlauf folgen. Die während einer Periode eingekoppelte berechnete Kolbenstrahlerenergie ist in diesem Vergleich am höchsten. In etwa in der gleichen Größenordnung, aber etwas niedriger, ist die in den maximal aufgeschwungenen Blasen gespeicherte Energie. Diese Beobachtung deckt sich mit der Tatsache, dass der Schalldruck in Kavitationsblasenfeldern durch die Blasen stark gedämpft wird und in Messungen um einiges niedriger ist als es mit der gemessenen Auslenkung des Schallwandlers zu erwarten wäre [14,64,87]. Die kinetische Energie des akustisch getriebenen Strömungsfeldes ist in diesen Betrachtungen am niedrigsten. Dies ist bei den verwendeten Frequenzen und den damit einher gehenden niedrigen Dämpfungsfaktoren $\mathrm{zu}$ erwarten. Allerdings ist zu erkennen, dass mit Überschreiten der Kavitationsschwelle die kinetische Energie stark ansteigt, was ebenso auf einen starken Anstieg des Dämpfungsfaktors und damit einen höheren Impulsübertrag von der Schallschnelle auf die Gleichströmung schließen lässt [15,84].

Die Absolutwerte der Energien sind hierbei als Angabe einer Größenordnung zu verstehen. Für die Kolbenstrahlerenergie wurde ein in einer unendlichen Wand eingebauter Kolbenstrahler mit $k a<<1$ ( $k$ Wellenzahl, a Durchmesser des Kolbenstrahlers) angenommen und die während einer Periode umgesetzte Leistung berechnet. Die Reflexion des Schalls an den Küvettenwänden wurde hier außer Acht gelassen. Die Berücksichtigung der Reflexionen hätte zur Folge, dass keine ebene Welle mehr angenommen werden könnte.

Für die Volumenenergie wurde die Energie aus der Differenz der maximal und minimal vorhandenen Anzahl der schwarzen Pixel berechnet. Bei dieser Methode ist allerdings schon das Grundrauschen, wie in einigen Abbildungen ohne Kavitation $\mathrm{zu}$ erkennen ist, relativ groß. Außerdem wurden einige, sicher nicht allgemeingültige, Annahmen zur Blasengrößenverteilung verwendet. Vor allem bei Änderung 
des Gasgehaltes oder der Temperatur ändert sich die Größe der Blasen. Trotzdem entspricht der Verlauf der Messwerte recht gut der Erwartung.

Die Berechnung der kinetischen Gesamtenergie wurde ebenfalls unter Annahme verschiedener Voraussetzungen durchgeführt. So wurde eine Rotationssymmetrie angenommen, die, wie in der spaltenweisen Auftragung der kinetischen Engergiedichte in Kapitel 4.6.1 zu erkennen ist, nicht immer perfekt gegeben ist. Außerdem wurde die gesamte im System gespeicherte Energie berechnet, die sich über viele Perioden aufsummiert hat. Allerdings wurde vor der eigentlichen Messung immer so lange gewartet, bis die Strömung konstant war. Es wurde demnach immer der eingeschwungene stationäre Zustand gemessen, weshalb sich die kinetischen Energien bei den einzelnen Spannungen vergleichen lassen.

\subsection{Detailaufgelöste Aufnahmen}

Die Aufnahmen mit höherer Bildrate zeigen sowohl bei hoher als auch bei niedriger Spannung ähnliche Effekte. Direkt an der Sonotrode spielt die rein akustische Strömung kaum eine Rolle. Durch den geringen Dämpfungsfaktor bei der verwendeten Frequenz wird hier kaum Schall absorbiert und in kinetische Energie dissipiert. Die Strömung wird hier fast ausschließlich durch die Kavitationsblasen - falls vorhanden - verursacht. Hierbei spielen Volumenoszillation, Oberflächenmoden und Bewegungen der Blasen eine Rolle. Hauptsächlich folgt die Strömung in diesem Bereich der Schwingung der großen Blase, beziehungsweise der Blasenwolke, an der Sonotrode. Die Schwingung dieser Blasen schiebt die Flüssigkeit beim Aufschwingen von der Sonotrode weg und saugt sie beim Kollabieren wieder auf die Sonotrode zu. Da diese Schwingung je nach Blasengröße zu einer anderen Phase des Schallfeldes erfolgt, sind die Werte recht stark gestreut. Dies ist sowohl in der Auftragung der schwarzen Pixel als auch in der Auftragung der mittleren vertikalen Geschwindigkeitskomponente zu erkennen. Im Aufschwingen der Blasen wird die Flüssigkeit von der Sonotrode weg beschleunigt und hat im maximal aufgeschwungenen Zustand ihr Maximum. Dann wird sie wieder auf die Sonotrode zu beschleunigt, um kurz nach dem Minimalvolumen die maximale Geschwindigkeit auf die Sonotrode zu zu erreichen. Die große Blase direkt an der Sonotrode scheint hierbei mit halber Frequenz zu schwingen, was sich in der Fouriertransformierten deutlich abzeichnet. Bei höheren Spannungen wird diese Periode-zwei-Schwingung durch die große Anzahl verschieden großer Blasen, die alle zu unterschiedlichen Phasen kollabieren, verdeckt und es tritt keine klare Spitze bei der halben Frequenz, sondern ein verbreiterter Bereich um die halbe Frequenz auf. 
Die hohe Aufnahmerate von 50000 Bps und die hohe räumliche Auflösung sind geeignet, um einzelne Strömungseffekte einzelnen Blasen zuordnen zu können. Allerdings ist dazu eine noch exaktere räumliche Kalibration der beiden Kameras nötig, was in dieser Arbeit noch nicht erreicht werden konnte. Trotzdem sind in den Aufnahmen in der Nähe von Blasen-Clustern außerhalb der an der Sonotrode anhaftenden großen Blase vereinzelt Strömungen zu erkennen, die sich eindeutig der Schwingung oder Bewegung der Blasen zuordnen lassen. Um die durch die Kollapsdynamik erzeugten Mikroströmungen um die Blasen zeitlich auflösen zu können, wären allerdings noch höhere Aufnahmeraten und eventuell kleinere Tracer-Partikel nötig.

\subsection{Einfluss des Schallfeldes auf die Strömung}

Die Simulation des Schallfeldes in der Küvette zeigt aufgrund des unsymmetrischen Aufbaus eine ebenfalls unsymmetrische Ausprägung. Dies könnte ein Grund für die immer wiederkehrende Asymmetrie des beobachteten Strömungsfeldes sein. Vor allem die Strömungsfelder bei Überdruck zeigen diese Asymmetrie. In diesem Fall wird der Schall nur schwach gedämpft und die Struktur des vorhandenen Schallfeldes mit eventuell vorhandenen Druckknoten, -bäuchen und Wanderwellenanteilen dominiert die entstehende Strömung. Tritt Kavitation auf, ändert sich das Schallfeld durch vorhandene Blasen und die dadurch resultierende Dämpfung massiv, und das ursprünglich vorhandene Schallfeld spielt keine wesentliche Rolle mehr. Wie schon erwähnt, beeinflussen sich Blasen und Schallfeld wechselseitig. Aus diesem Grund ist die Richtung der Strömung im Kavitationsblasenfeld sehr instabil, fluktuiert und die Auswahl des Bildes beeinflusst die abgebildete Strömungsrichtung. Der lineare Anstieg des Drucks mit der Auslenkung der Sonotrode und der starke Abfall mit dem Abstand wurde in Messungen bestätigt [64]. In der Realität wird der Abfall des Schalldruckes direkt vor der Sonotrode durch das Einsetzen von Kavitation noch steiler ausfallen. 


\section{Kapitel 7 Untersuchung von Strömungen im Gigahertzbereich}

Die Strömungen im Gigahertzbereich (GHz-Bereich) wurden an zwei verschiedenen speziell entwickelten Schallwandlern untersucht. Hierbei handelt es sich um einen Array-Schallwandler, der von der Firma TRIQuint SEmiconDUCTOR, INC. entwickelt und produziert wurde, und um einen ZwillingsSchallwandler der von der École polytechnique fédérale de Lausanne (EPFL) entwickelt und produziert wurde. Die Array-Schallwandler wurden im Dauerbetrieb untersucht. Wegen der sehr kleinen Wandlerfläche und der dadurch recht hohen Leistungsdichte wurden die Zwillings-Schallwandler im amplitudenmodulierten Betrieb untersucht.

\subsection{Messungen und Methodik}

\subsubsection{PIV-Messungen und Tintenfront-Verfolgung}

Die Form der Strömungen über den Gigahertzschallwandlern (GHzSchallwandler) und ihre Geschwindigkeit wurde mit Hilfe von zwei Methoden untersucht. Um die Geschwindigkeit der Strömung zu bestimmen, wurden PIVMessungen durchgeführt. Hierzu wurde die Küvette mit DI-Wasser gefüllt und es wurden Fluoreszenzpartikel mit einem oder zehn Mikrometer Größe hinzugefügt. Diese Strömung wurde mit einer Hochgeschwindigkeitskamera (PHOTRON SA5) mit verschiedenen Bildraten aufgenommen und mit PIVLab ausgewertet. Für diese Auswertung wurden verschieden große Auswertungsfenster und Verschiebungen (siehe Kapitel 4.1.2) verwendet. Eine Bearbeitung der Bilder war größtenteils nicht nötig. In einigen Fällen, wenn die Tiefenschärfe zu groß oder der Kontrast zu gering war, wurde ein Algorithmus zum Finden der Kanten verwendet, um nur scharfe Partikel in der Auswertung zu betrachten. Die erhaltenen Geschwindigkeitsfelder wurden mit MATLAB weiterverarbeitet. 
Um die Form der Strömung vor allem im gepulsten Betrieb sichtbar zu machen, wurde die Methode der Tintenfront-Verfolgung angewendet. Dazu wurde vor dem Anschalten der Schallwandler mit einer Mikroliterpipette vorsichtig ein Tropfen Tinte direkt auf den Wandlerchip in das DI-Wasser gegeben. Anschließend wurde der Wandler eingeschaltet und währenddessen die Aufnahme gemacht. Für eine erneute Aufnahme wurde das Tinte-Wasser-Gemisch entfernt, die Küvette gereinigt und mit neuem Wasser und gegebenenfalls neuer Tinte befüllt.

\subsubsection{Elektrochemische Messungen}

Um eine relative Geschwindigkeitsverteilung innerhalb des erzeugten Strömungsfeldes bestimmen zu können, wurden Chronoamperometriemessungen mit dem in Kapitel 3.2.2 beschriebenen Aufbau durchgeführt. Die verwendeten Verschiebetische lassen sich mit Hilfe der mitgelieferten Software in einem automatisierten Modus betreiben. Die Schrittweite, die Verzögerung zwischen den Schritten und die Geschwindigkeit der Bewegung sind einstellbar. Für die Messungen in x-, y- oder z-Richtung wurden verschiedene Schrittweiten gewählt und an jeder Position wurde $1 \mathrm{~s}$ bis zum nächsten Schritt gewartet. Währenddessen wurde die Chronoamperometriemessung kontinuierlich durchgeführt. Zwei der gemessenen Kurven sind in Abbildung 7.1 gezeigt. Durch die konstante Zeitschrittlänge, konnte die erhaltene Kurve über der Zeit anschließend mit MatLaB in eine Kurve über der Position der Elektrode umgerechnet werden. Hierzu wurde ein etwas kleinerer Zeitbereich aus der Mitte der Ruhezeit der Elektrode gewählt, um die Randeffekte durch die Bewegung der Elektrode auszuklammern. Durch den gepulsten Betrieb schwankt die erhaltene Messkurve stark, weshalb innerhalb des gewählten Zeitbereichs mit MATLAB die lokalen Maxima gesucht und über diese gemittelt wurde. 


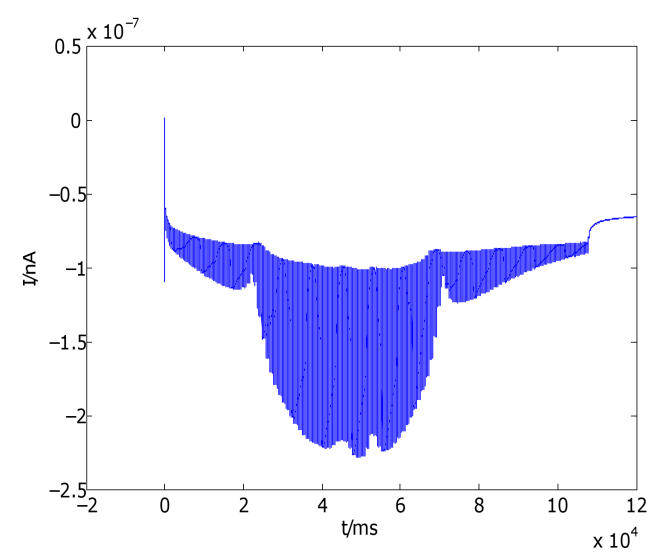

(a) Originaldaten einer Elektrochemiemessung eines Zwillings-Schallwandlers in $y$ Richtung.

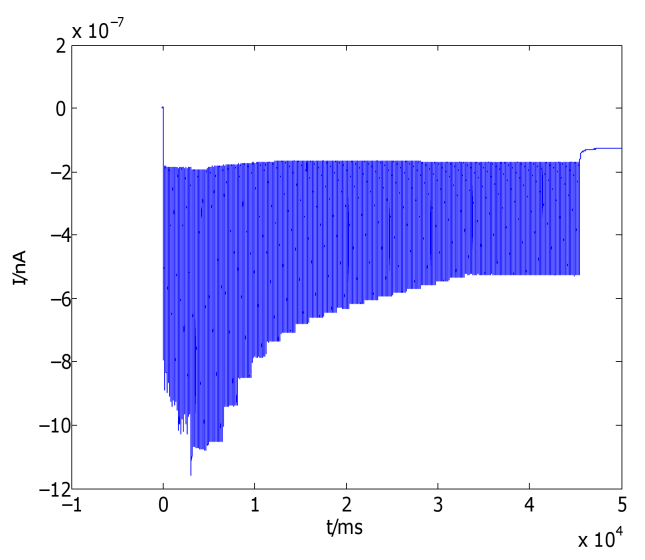

(b) Originaldaten einer Elektrochemiemessung eines Zwillings-Schallwandlers in $\mathrm{Z}$ Richtung.

Abbildung 7.1.: Rohdaten der Elektrochemiemessungen. Die breite Form der Kurve kommt durch den Pulsbetrieb des Wandlers zustande, die Schritte des Verschiebetisches sind gut zu erkennen.

\subsection{Untersuchung von akustischen Strömungen an einem Array-Schallwandler}

Die Strömungen an dem Array-Schallwandler wurden mit verschiedenen Methoden untersucht. PIV-Aufnahmen mit Hintergrundbeleuchtung wurden im stationären Zustand der Strömung durchgeführt, um die Strömungsgeschwindigkeiten messen zu können. Außerdem wurden der Anschaltvorgang und das Entstehen der Jetströmung mit Hilfe von Partikeln und Tinte direkt auf dem Wandler untersucht. Hierzu wurden Partikel von $10 \mu \mathrm{m}$ Größe verwendet, um die Partikel auch bei einem großen Bildausschnitt, bei dem die gesamte Küvette sichtbar ist, auflösen zu können. Um den Anschaltvorgang sichtbar zu machen, wurde die Tinte vorsichtig mit Hilfe einer Mikroliterpipette direkt auf dem Wandlerchip platziert. Da die Partikel eine minimal höhere Dichte als Wasser aufweisen, musste in diesem Fall nur gewartet werden, bis sich die Partikel abgesetzt hatten. So konnte der Anschaltvorgang mit der sich ausbreitenden Strömungsfront und den Wirbeln innerhalb der Strömung sichtbar gemacht werden. 


\subsubsection{Großräumige Strömungen in der Küvette}

Abbildung 7.2 zeigt das Strömungsfeld innerhalb der gesamten Küvette (siehe auch [107]). Der Wandler mit etwa $3 \mathrm{~mm}$ Breite befindet sich am unteren Rand des Bildes. Die Geschwindigkeit ist durch die Farbcodierung, die Richtung der Strömung durch die normierten Pfeile gezeigt. In der Mitte der Küvette ist eine Jet-artige Strömung senkrecht nach oben zu erkennen, die im oberen Bereich auf die Wasseroberfläche trifft und zu beiden Seiten gelenkt wird. An den Wänden der Küvette ist die Strömung nach unten gerichtet und im oberen Bereich sind links und rechts je ein Wirbelzentrum zu erkennen. Die maximale Strömungsgeschwindigkeit wurde auf etwa der halben Höhe der Küvette gemessen und beträgt etwa $4.1 \mathrm{~cm} \mathrm{~s}^{-1}$. Laut der Theorie nach LighthilL (siehe Kapitel 2.1.3) sollte die maximale Strömungsgeschwindigkeit nahe des Schallwandlers noch im Bereich des gedämpften Schalls auftreten. Bei diesen Frequenzen und der daraus resultierenden hohen Dämpfung sollte dies also in einigen zehn Mikrometern Entfernung zu beobachten sein. Aufgrund der verwendeten Aufnahmerate und Auflösung kann die Strömung nahe des Wandlers in dieser Aufnahme leider nicht aufgelöst werden. Auf die Strömung nahe am Wandler wird in den folgenden Kapiteln noch näher eingegangen.

Abbildung 7.3 zeigt eine mit Tinte sichtbar gemachte Strömung nach dem Einschalten. Es ist zu sehen, dass sich eine pilzförmige Struktur nach oben bewegt und sich an der Oberfläche des Wassers zu den Seiten ausbreitet. Dabei wird der entstehende Jet leicht nach rechts abgelenkt. Nachdem die verbreiterte Struktur an der Oberfläche angekommen ist, bildet sich ein gleichförmiger stabiler Jet aus. Bei genauer Betrachtung sind die leicht ausgefransten Ränder des Jets zu erkennen, was eventuell auf Eigenschaften der Tinte zurückzuführen ist. Aufgrund der abnehmenden Menge an Tinte direkt am Wandler wird der Jet mit der Zeit etwas schmaler. Da eine durch thermische Konvektion entstehende Strömung ähnlich aussehen würde, wurde durch Verkippen des Wandlers verifiziert, dass es sich tatsächlich um akustisch getriebene Strömungen handelt. Der entstehende Jet bleibt auch bei starker Verkippung des Wandlers senkrecht zu diesem.

Den gleichen Vorgang zeigt Abbildung 7.4 mit Partikeln, die sich auf der Wandleroberfläche abgesetzt haben. Die innere Struktur des verbreiterten Bereichs (Pilzkopf) ist deutlich zu erkennen. Außerdem ist auf den ersten Bildern zu sehen, dass sich zuerst an den Rändern des aktiven Bereichs kleinere Wirbel bilden und sich erst nach einiger Zeit zu einer größeren Strömung zusammenschließen. Aufgrund der geringen Partikeldichte ist der entstehende Jet hier kaum zu erkennen. 


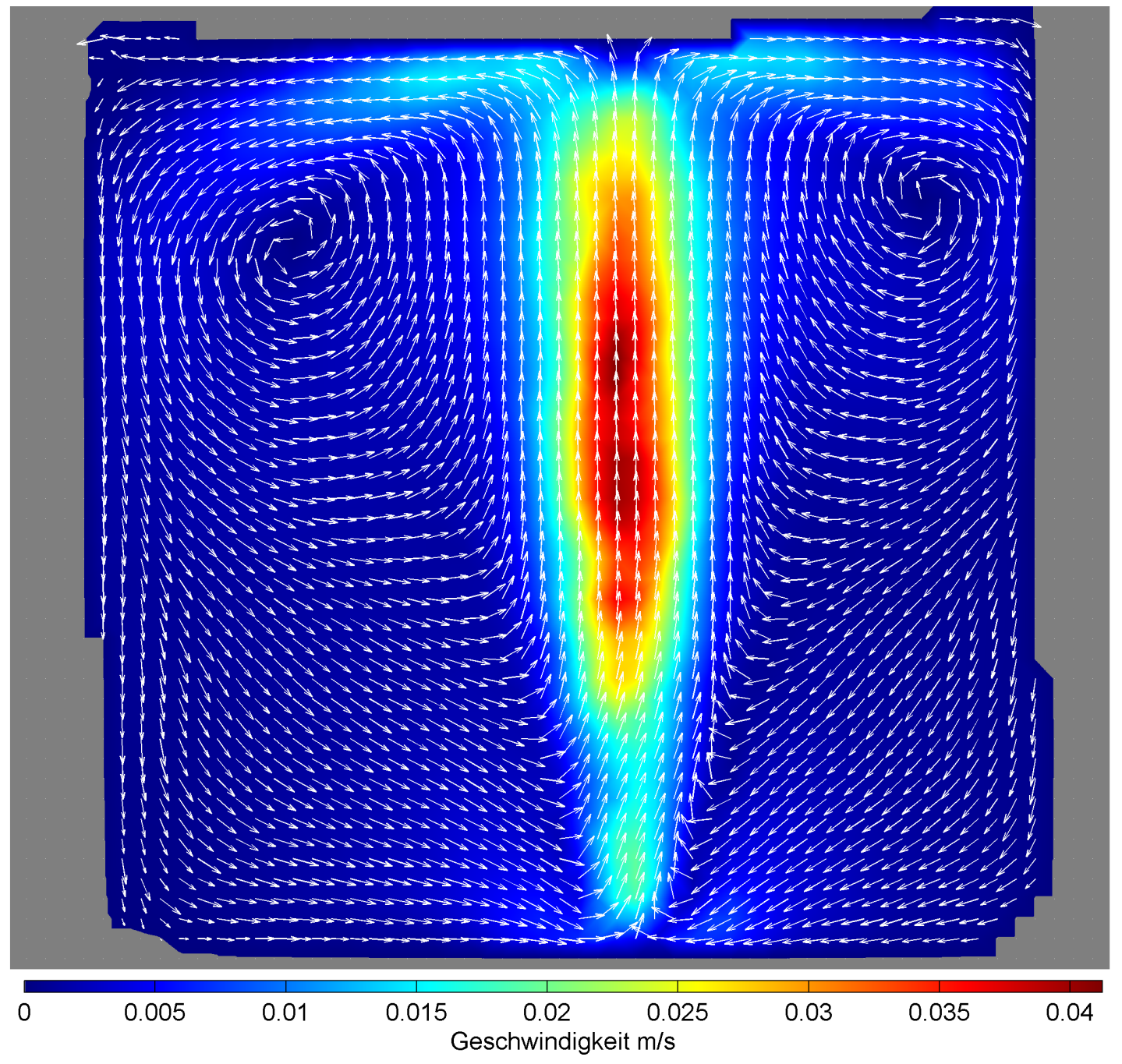

Abbildung 7.2.: Zeitgemittelte PIV-Messung der Strömung über einem Array-Schallwandler. Die Breite des farbcodierten Bereiches beträgt etwa $1 \mathrm{~cm}$, der Wandler befindet sich am unteren Rand und ist etwa $3 \mathrm{~mm}$ breit. Grafik entnommen aus [107].

\subsubsection{Wirbel an der Wandleroberfläche}

In diesem Kapitel wird nun näher auf die direkt am Wandler entstehenden Strömungen eingegangen (siehe auch [107] und [108]). Abbildung 7.5 zeigt eine PIVAuswertung dieser Strömung. Die maximale Strömungsgeschwindigkeit liegt hier bei $13 \mathrm{~cm} \mathrm{~s}^{-1}$ und wird etwa $500 \mu \mathrm{m}$ über der Wandleroberfläche im linken Bereich der Strömung erreicht. Nach oben hin wird das Strömungsfeld leicht breiter und die Geschwindigkeit nimmt etwas ab. Links und rechts des Strömungsfeldes direkt am Wandler sind kleinere Bereiche schnellerer Strömung zu erkennen. Hier 


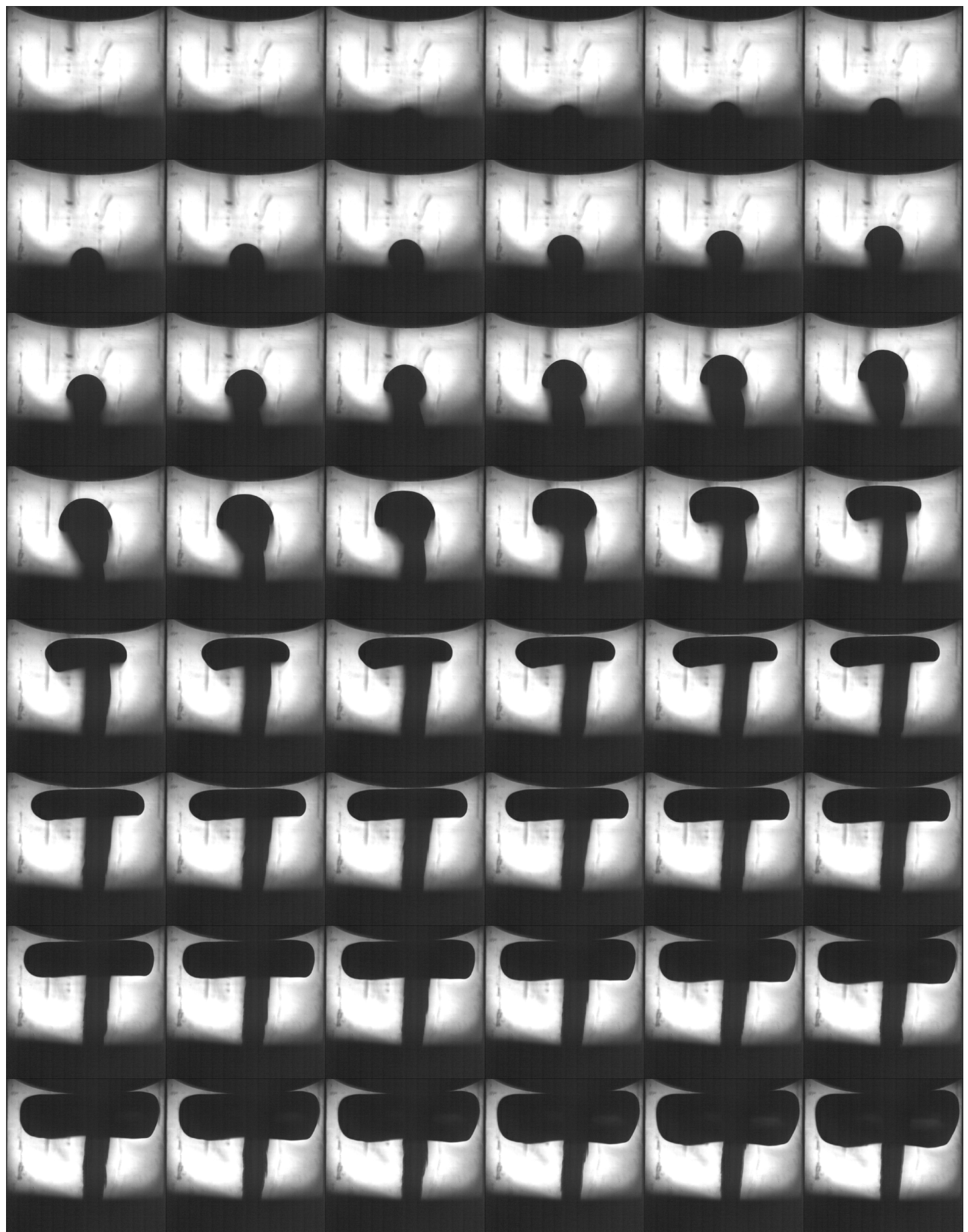

Abbildung 7.3.: Aufsteigende Strömung nach Einschalten des ArraySchallwandlers bei $1.8947 \mathrm{GHz}$. Der Film wurde mit 125 Bps (Bilder pro Sekunde) aufgenommen, abgedruckt ist jedes zehnte Bild. Die Breite eines Einzelbildes beträgt etwa $1 \mathrm{~cm}$. 


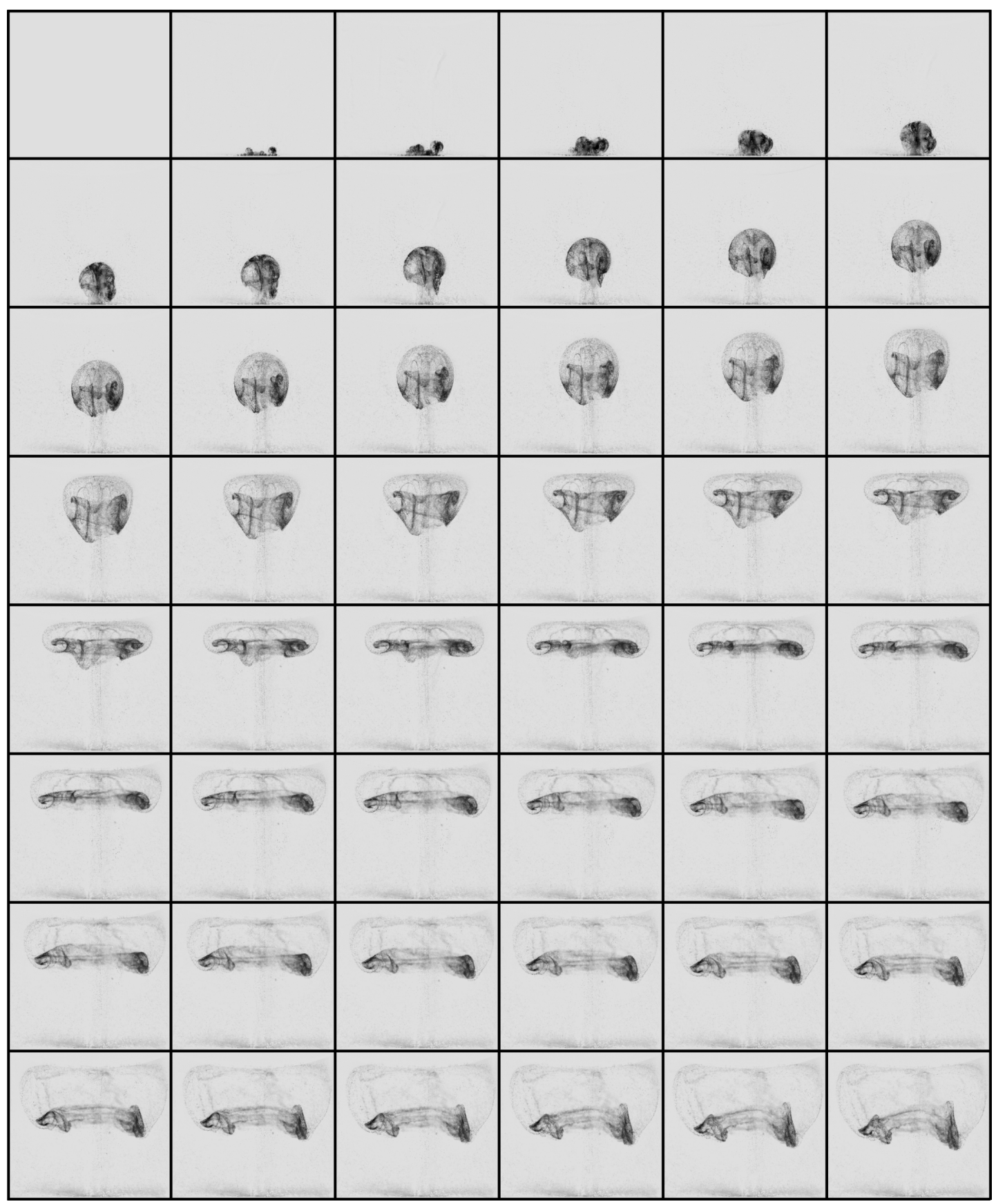

Abbildung 7.4.: Aufsteigende Strömung nach Einschalten des ArraySchallwandlers bei $1.8947 \mathrm{GHz}$. Der Film wurde mit 125 Bps aufgenommen, abgedruckt ist jedes zehnte Bild. Die Breite eines Einzelbildes beträgt etwa $1 \mathrm{~cm}$ 


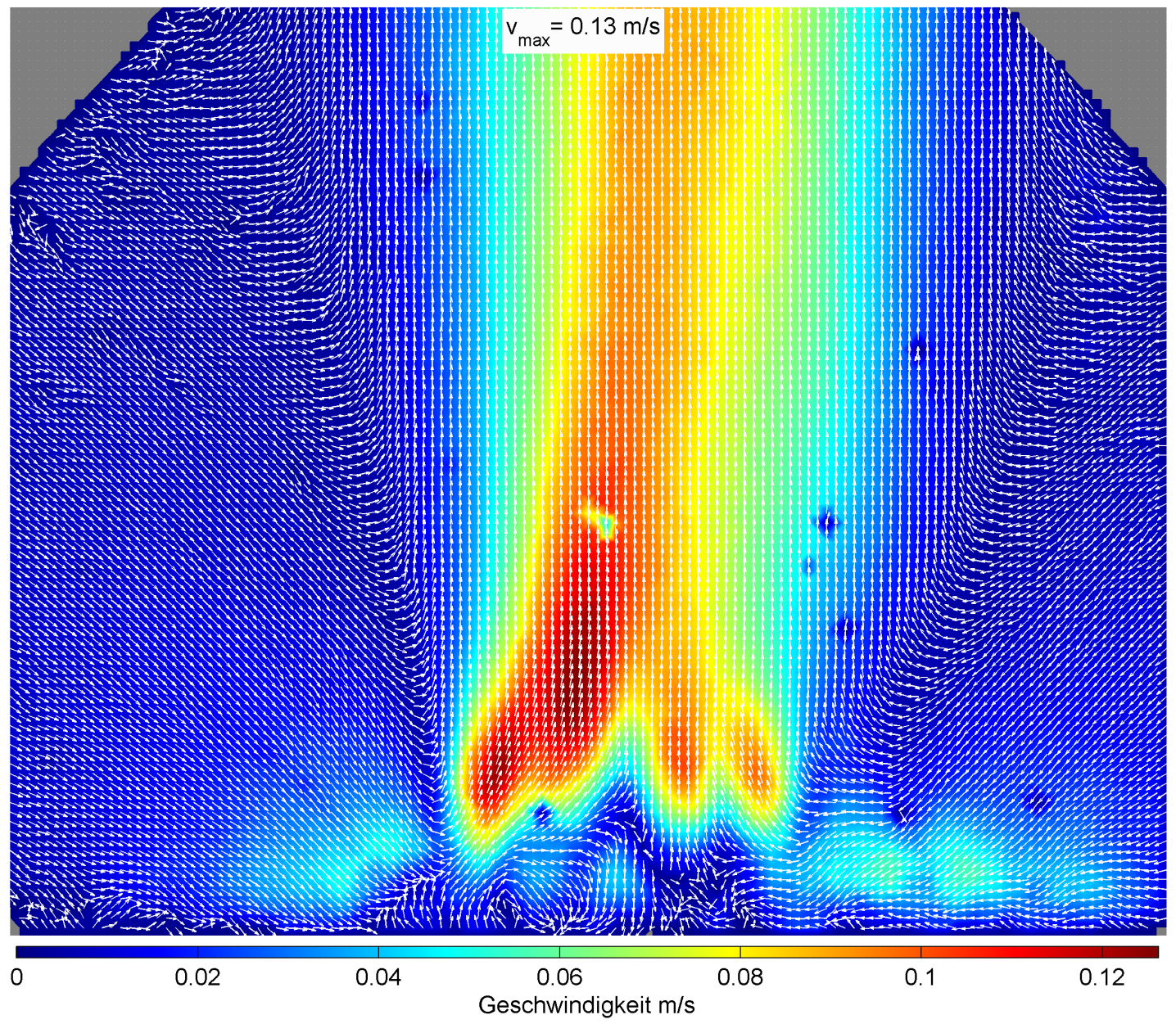

Abbildung 7.5.: Zeitgemittelte PIV Messung der Strömung über einem Array-Schallwandler. Die treibenden Wirbel sind im unteren Bereich des Bildes zu erkennen. Die Breite des Bildes beträgt etwas weniger als $3 \mathrm{~mm}$, es ist also fast der komplette Wandler abgebildet. Grafik nach [107] und [108].

wird die Flüssigkeit durch die einzelnen Wandler zur Mitte des Wandlers befördert. In der Mitte des Bildes, die auch die Mitte des Wandlers darstellt, ist eine Rückströmung auf den Wandler zu zu erkennen. Auch in den äußeren Bereichen ist die Strömung in größerer Entfernung auf den Wandler zu und mit weiterer Annäherung an den Wandler immer mehr zur Mitte gerichtet.

Die Abbildungen 7.6(a) und 7.6(b) zeigen mit Hilfe von Rohdatenbildern der Durchlichtaufnahmen die schon erwähnten Wirbel direkt an der Wandleroberfläche. Abbildung 7.6(a) zeigt den Bereich über der Wandleroberfläche. Durch darin gefangene Partikel sind kleine Ringwirbel mit etwa 50-100 $\mu \mathrm{m}$ Durchmesser zu erkennen. Außerdem sind im unteren Bereich des Bildes etwas heller erscheinende 
Bereiche zu sehen, die wahrscheinlich durch thermische Effekte und somit Lichtbrechung direkt über den Einzelwandlern hervorgerufen werden. Eine Großaufnahme eines einzelnen Wirbelrings ist in Abbildung 7.6(b) zu sehen. Dieser Ring hat in der Höhe etwas mehr als 100 in der Breite aber weniger als 100 um Durchmesser.

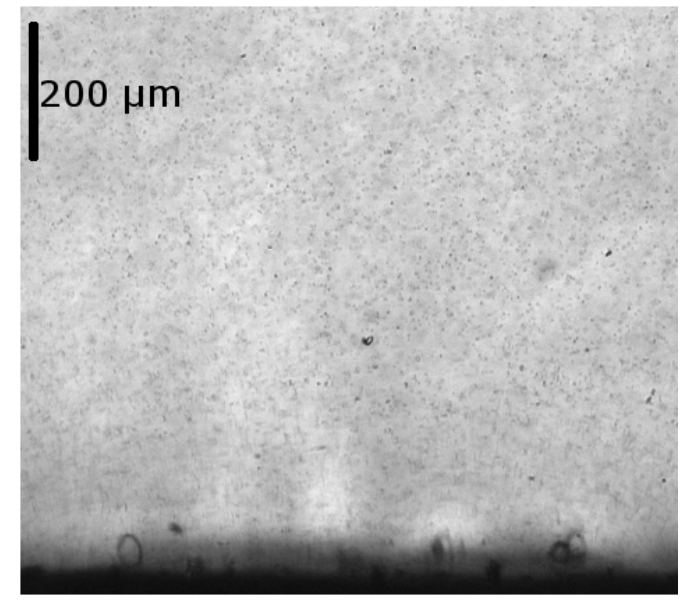

(a) Übersicht über die Wandleroberfläche. Die Wirbelringe sind im unteren Bereich sichtbar. Die helleren Regionen sind thermische Effekte. Grafik aus [108].

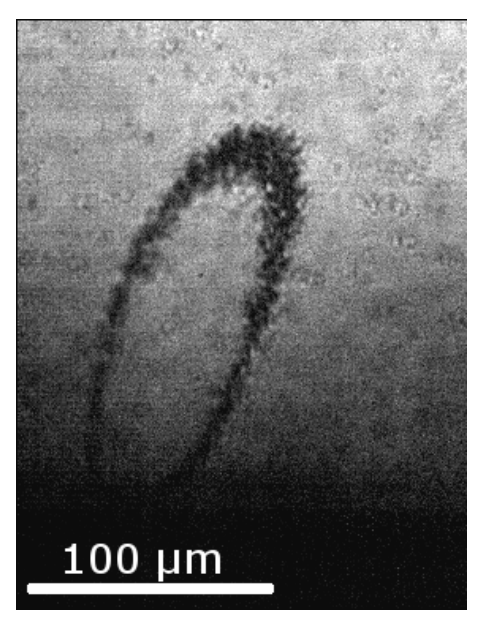

(b) Einzelner Wirbelring an der Wandleroberfläche.

Abbildung 7.6.: Wirbelringe an der Wandleroberfläche eines ArraySchallwandlers, sichtbar gemacht durch darin eingeschlossene Partikel. Der Wandler wurde mit $1.902 \mathrm{GHz}$ betrieben.

\subsubsection{Elektrochemische Messung}

Um die Strömungen an dem Array-Schallwandler qualitativ genauer zu untersuchen, wurden Elektrochemiemessungen durchgeführt (siehe auch [107]). Basis dieser Messungen ist die Annahme, dass der gemessene Strom proportional zur lokalen Strömungsgeschwindigkeit ist. In Abbildung 7.7 ist die Auftragung des gemessenen Stroms über der Entfernung vom Schallwandler gezeigt. Hierzu wurden vier Messungen durchgeführt, um die Reproduzierbarkeit der Messung zu überprüfen. Die Elektrode wurde direkt auf dem Wandler aufgesetzt und in $5 \mu \mathrm{m}$-Schritten vom Wandler weg bewegt $(0-100 \mu \mathrm{m})$. Es ist $\mathrm{zu}$ sehen, dass der Strom erst stark ansteigt, bei $20 \mu \mathrm{m}$ Abstand ein Maximum hat und dann linear wieder abfällt. Anschließend wurde die Elektrode wieder an den Wandler angenähert $(100-0 \mu \mathrm{m})$. Hierbei ist ein leicht von der vorherigen Messung verschiedener Verlauf zu erkennen. Bei der Annäherung steigt der Strom erst an, hat bei etwa 
$45 \mu \mathrm{m}$ Abstand ein Maximum, bleibt dann konstant und fällt ab ungefähr $15 \mu \mathrm{m}$ Abstand steil ab. Die Wiederholungsmessungen liegen in beiden Richtungen gut auf den zuerst gemessenen Kurven. Die Messungen legen nahe, dass die für die gemessene Geschwindigkeit verantwortlichen Wirbel sich abhängig von den Anfangsbedingungen ausbilden. So verformt die sich annähernde Elektrode (Grenzfläche) die bestehenden Wirbel erst, bevor sie sich durch die akustische Grenzschicht nicht mehr ausbilden können und ganz unterdrückt werden. Bei der Entfernung der Elektrode vom Wandler muss ein bestimmter Abstand erreicht sein, damit sich die Wirbel ausbilden können.

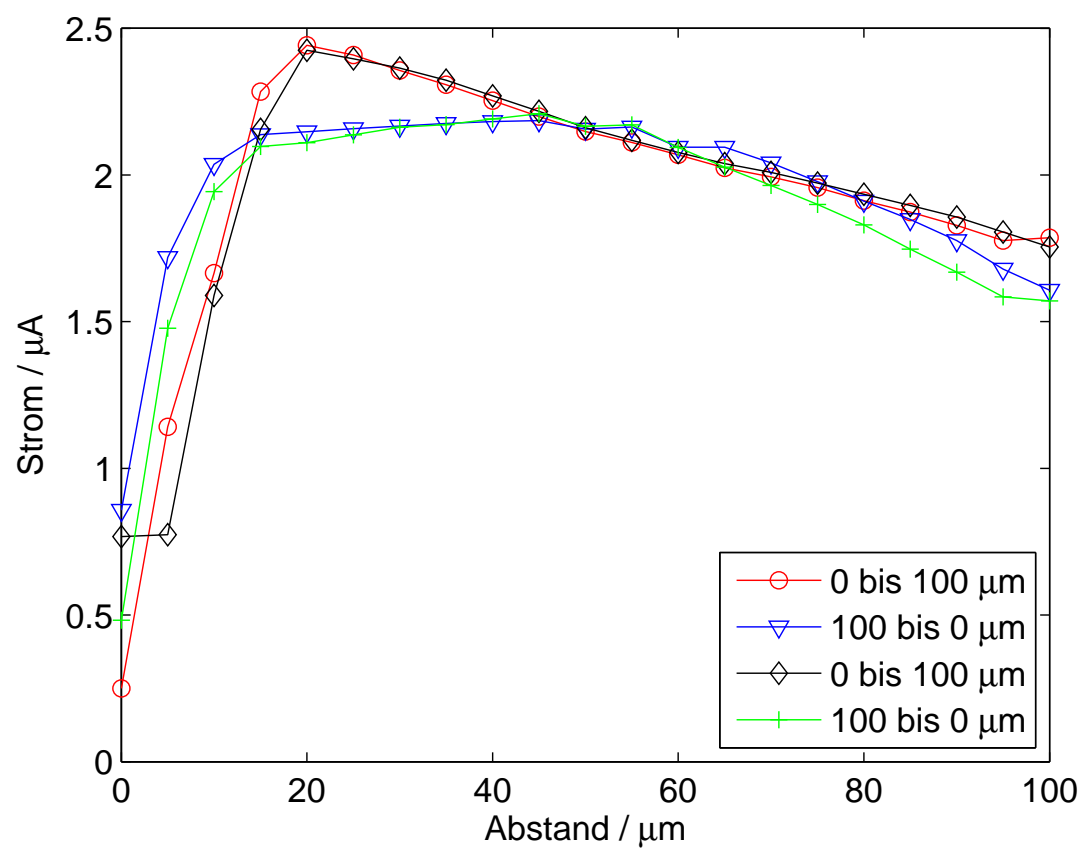

Abbildung 7.7.: Vergleich von vier Messungen des Stromes in Abhängigkeit vom Abstand der Messelektrode vom Wandler bei Entfernung und Annäherung vom Array-Schallwandler.

Abbildung 7.8 zeigt einen Scan entlang der $x$-Richtung des Wandlers. Diese Messung wurde einmal über die gesamte Breite der Küvette und einmal über die Breite der aktiven Fläche des Wandlers mit erhöhter Auflösung durchgeführt. Bei diesen Messungen sind mehrere wichtige Aspekte zu erkennen. So steigen beide Kurven von links nach rechts leicht an. Dies liegt an einer Erhöhung der Temperatur während der Messung, die die Reaktionsrate des elektrochemischen Prozesses beeinflusst. Bei einer Erhöhung der Temperatur nimmt die Reaktions- 
rate zu. Außerdem ist die Array-Struktur des Wandlers mit den 15 einzelnen Wandlern zu erkennen. Diese ist in beiden Messungen abgebildet, nur rechts der Wandlermitte weichen beide Messungen leicht voneinander ab. Die Messungen wurden in einem Abstand von $130 \mu \mathrm{m}$ der Messelektrode vom Schallwandler durchgeführt. Außerdem fällt auf, dass der gemessene Strom in der Mitte über dem Array am höchsten ist. Dies könnte ebenfalls in der Beeinflussung der Wirbel durch die Elektrode begründet liegen. Der Flüssigkeitsaustausch in der Spalte zwischen Wandler und Grenzfläche scheint am besten zu funktionieren, wenn die Elektrode symmetrisch über dem Wandler positioniert ist. In dieser Messung ist der Durchmesser der Elektrode außerdem kleiner als die Abmessungen des gesamten Arrays, was dazu führt, dass bei mittiger Positionierung die Strömung symmetrisch an den Seiten der Elektrode nach oben strömen kann.

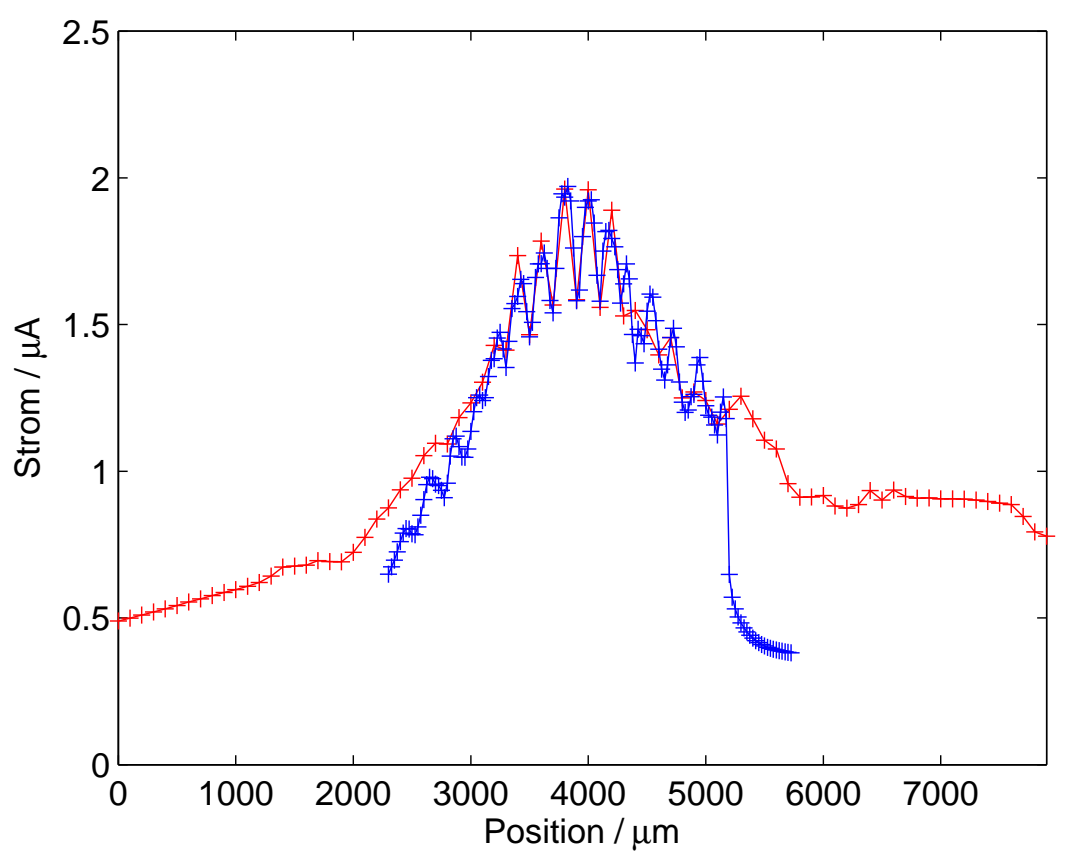

Abbildung 7.8.: Messungen des Stromes in Abhängigkeit von der Position in $x$-Richtung über dem Array-Schallwandler. Einmal mit geringer Auflösung über die gesamte Breite der Küvette und einmal mit hoher Auflösung nur im Bereich des Wandlers. Die Elektrode wurde in der Entfernung mit dem größten Messsignal von $130 \mu \mathrm{m}$ parallel zur Blickrichtung der Kamera aus den PIV-Aufnahmen bewegt. 
Die $y$-Richtung des Wandlers wurde ebenfalls vermessen und ist in Abbildung 7.9 aufgetragen. Diese Messung wurde mit einer noch höheren Auflösung von $25 \mu \mathrm{m}$ durchgeführt. Genau wie in der $x$-Richtung sind die 15 einzelnen Wandler des Arrays gut zu erkennen. Die Intensität zwischen den einzelnen Wandlern schwankt leicht, was an einer Fluktuation der Schichtdicken durch den Produktionsprozess und damit der Resonanzfrequenzen liegen könnte. Die Zunahme des Stromes durch die Zunahme der Temperatur während der Messung ist hier ebenfalls zu erkennen.

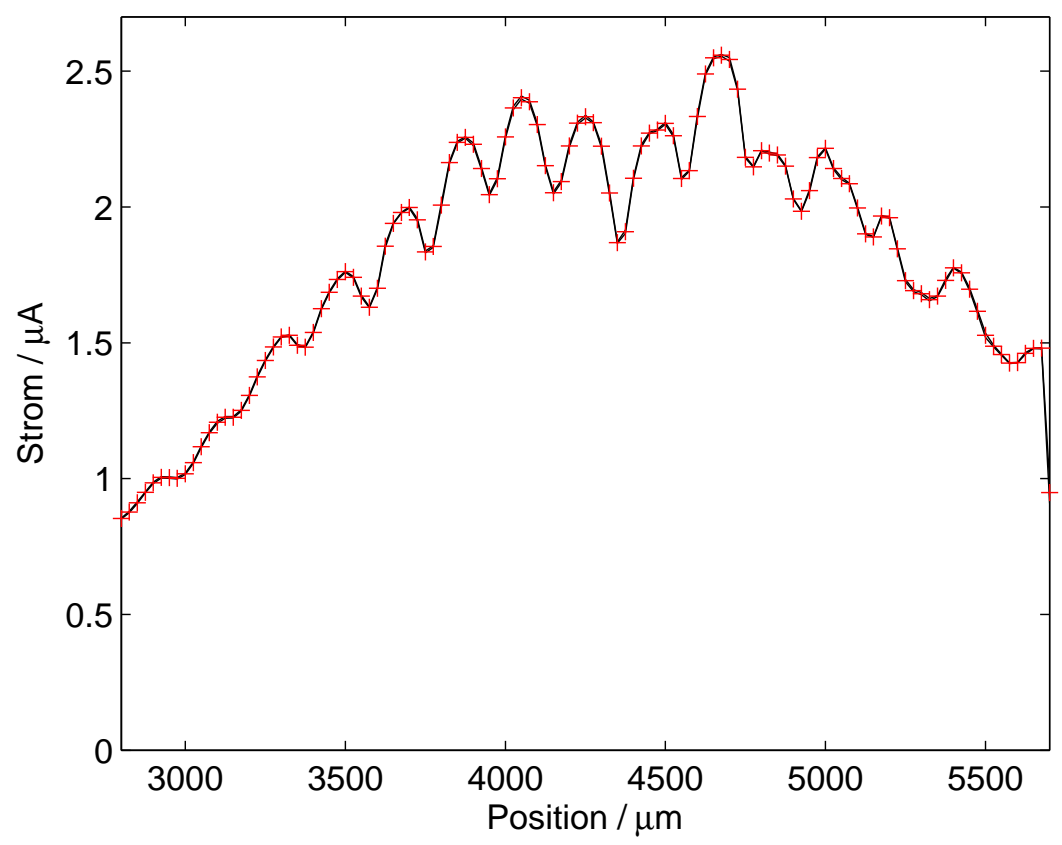

Abbildung 7.9.: Messungen des Stromes in Abhängigkeit von der Position in $y$-Richtung über dem Array-Schallwandler. Die Elektrode wurde in der Entfernung in der der maximale Strom gemessen wurde $(130 \mu \mathrm{m})$ senkrecht zur Blickrichtung der Kamera aus den PIV-Aufnahmen bewegt.

Abbildung 7.10 zeigt eine noch höher aufgelöste Messung der Strömungsabhängigkeit vom Abstand der Messelektrode von der Wandleroberfläche. Hier wurde die Messung für die ersten $10 \mu \mathrm{m}$ mit einer Auflösung von $100 \mathrm{~nm}$ durchgeführt und über der Anzahl der Wellenlängen bei dieser Frequenz in Wasser aufgetragen. Sowohl bei Entfernung als auch bei Annäherung an den Wandler sind leichte Fluktuationen des gemessenen Stromes zu erkennen. Diese lassen sich eventuell auf Stehwelleneffekte zwischen Wandler und Elektrode zurückführen. Allerdings lassen sie sich nicht genau in die Größe der berechneten Wellenlänge einordnen. 


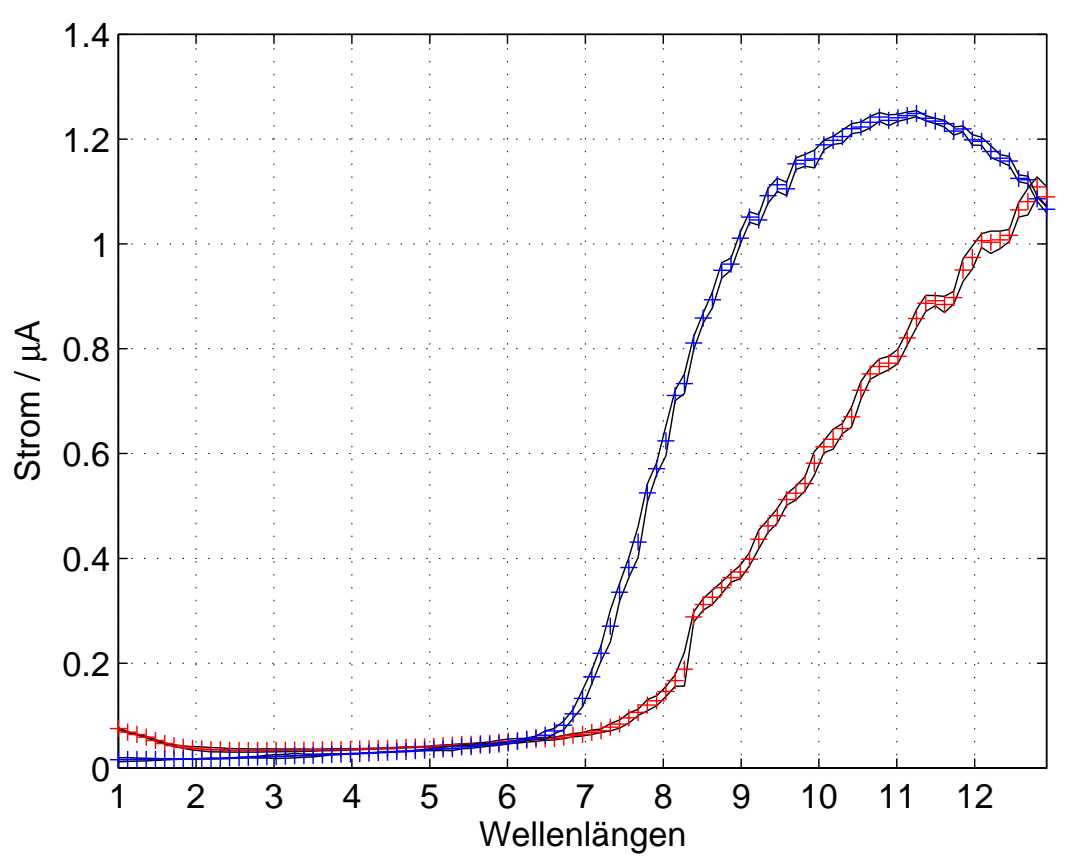

Abbildung 7.10.: Messungen des Stromes in Abhängigkeit von der Position in z-Richtung über dem Array-Schallwandler. Die Änderung der Strömung, wahrscheinlich durch stehende Wellen, ist gut zu erkennen.

\subsection{Untersuchung von akustischen Strömungen an einem Zwillings-Schallwandler}

Die Strömung an den Zwillings-Schallwandlern wurde mit verschiedenen Methoden untersucht. So wurden Aufnahmen des Anschaltvorgangs mit Tinte direkt über dem Wandler gemacht, um die Struktur der Strömung sichtbar zu machen. Außerdem wurden PIV-Aufnahmen mit Hintergrundbeleuchtung durchgeführt, um die Strömungsgeschwindigkeiten messen zu können. Hierzu wurden Partikel von $10 \mu \mathrm{m}$ Größe verwendet, um die Partikel auch bei großem Bildausschnitt in der Größe der gesamten Küvette sehen zu können. Diese Messungen wurden systematisch für verschiedene Leistungen und Betriebsarten durchgeführt. Außerdem wurden elektrochemische Messungen gemacht, um die Strömung direkt am Wandler qualitativ zu untersuchen. 


\subsubsection{Elektrische Pulsformen und Leistung}

Um die Zwillings-Schallwandler elektrisch und thermisch nicht zu überlasten, wurden diese amplitudenmoduliert betrieben, da der Frequenzgenerator keinen echten Pulsbetrieb erlaubte. Es wurde eine Amplitudenmodulation von 100\% gewählt, so dass am Wandler in den Phasen niedriger Amplitude keine Spannung anliegen sollte - auf die tatsächliche Signalform wird im Folgenden genauer eingegangen. Es wurden Modulationsfrequenzen von 10, 100 und $1000 \mathrm{~Hz}$ gewählt und die am Wandler anliegende Spannung wurde gemessen. Hierzu wurden mehrere Dämpfungsglieder mit insgesamt $19 \mathrm{~dB}$ Dämpfung in die Leitung gesetzt und mit Hilfe eines Oszilloskops mit $50 \Omega$ Eingangswiderstand wurde die Spannung gemessen. Um die Pulsformen gut darstellen zu können wurde eine geringe Sampling-Rate gewählt, so dass die eigentliche Sinusschwingung von $1.83 \mathrm{GHz}$ unterabgetastet wurde. Mit Hilfe von MatLaB wurden die gemessenen Kurven in einzelne Bereiche unterteilt, in denen die lokalen Maxima gesucht und gemittelt wurden. Diese erhaltenen Werte sind in Abbildung 7.11 aufgetragen. Es ist also der positive Teil der Einhüllenden mit der Trägerfrequenz von $1.83 \mathrm{GHz}$ zu sehen. Die hier aufgetragenen Messungen wurden alle bei der maximal verwendeten Leistung von $6 \mathrm{dBm}$ Ausgangsleistung des Frequenzgenerators und 1\% Einstellung des Potentiometers am Verstärker gemacht. Bei unmoduliertem Betrieb ergibt sich nun eine Spannungsamplitude von im Mittel 12.5 V (Teilabbildung 7.11(a)). Wird das Signal mit $10 \mathrm{~Hz}$ moduliert, ergibt sich vor Beginn des eigentlichen Pulses ein Einschwingvorgang auf bereits $12.5 \mathrm{~V}$. Zu Beginn des Pulses folgt dann ein starker Sprung auf die Spitzenspannung von knapp $33 \mathrm{~V}$, gefolgt von einem Abklingen der Spannung auf wieder $12.5 \mathrm{~V}$ bis zum Ende des Pulses. Dann fällt die Spannung steil auf $0 \mathrm{~V}$ ab (Abbildung 7.11(b)).

Bei $100 \mathrm{~Hz}$ Amplitudenmodulationsfrequenz (Teilabbildung 7.11(c)) ist wieder ein leichter Anstieg der Spannung schon im Zeitraum vor dem Einsetzen des eigentlichen Pulses zu erkennen. Hier steigt die Spannung allerdings nur auf etwa $2.5 \mathrm{~V}$ an. Der darauf folgende Sprung lässt die Spannung auf etwa $26 \mathrm{~V}$ ansteigen. Im Anschluss sinkt sie bis zum Ende des Pulses auf $24 \mathrm{~V}$ ab, um am Ende des Pulses wieder steil auf knapp über $0 \mathrm{~V}$ zu fallen.

Der beste Rechteckpuls wird bei $1000 \mathrm{~Hz}$ Modulationsfrequenz, zu sehen in Teilabbildung 7.11(d), erreicht. Allerdings ist die Spannung hier im Bereich außerhalb des Pulses nicht bei 0, sondern bei etwas über $1 \mathrm{~V}$. Während des eigentlichen Pulses steigt die Spannung auch hier noch auf das Doppelte der unmodulierten Spannung, auf etwa $25 \mathrm{~V}$, an.

Diese Pulsformen haben mehrere Ursachen. So ist der Frequenzgenerator für Frequenzen zwischen einigen $\mathrm{MHz}$ und vier $\mathrm{GHz}$ ausgelegt und scheint mit den nied- 


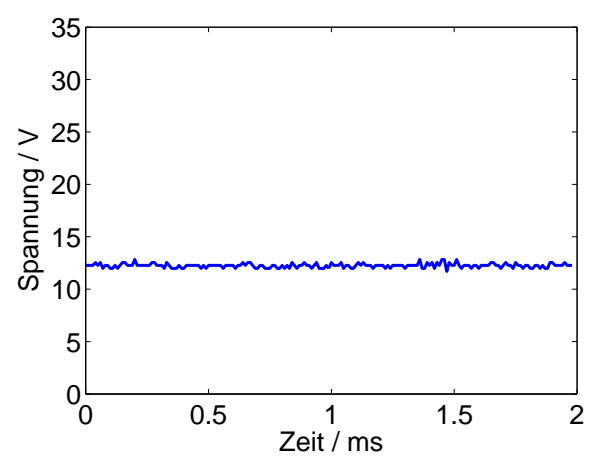

(a) Pulsform des elektrischen Signals ohne Amplitudenmodulation

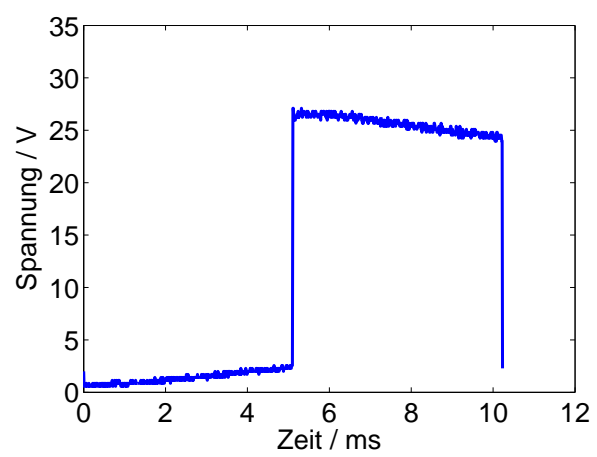

(c) Pulsform des elektrischen Signals bei $100 \mathrm{~Hz}$.

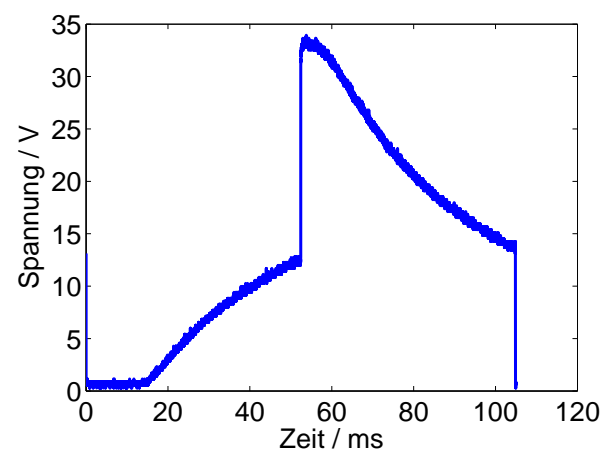

(b) Pulsform des elektrischen Signals bei $10 \mathrm{~Hz}$. Dieses Signal weist die höchste Spitzenleistung der verwendeten auf.

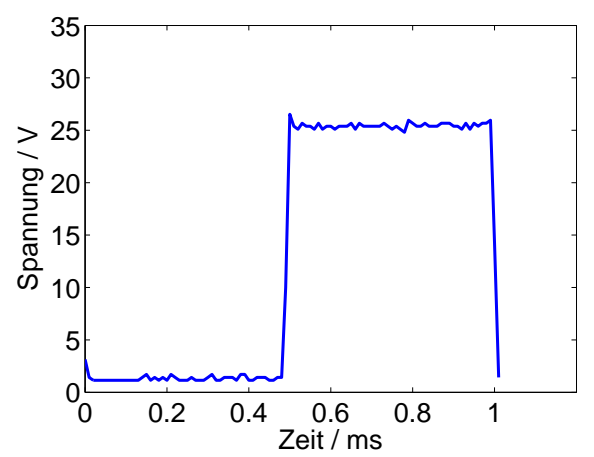

(d) Pulsform des elektrischen Signals bei $1000 \mathrm{~Hz}$.

Abbildung 7.11.: Einhüllende der Amplitudenmodulation bei $6 \mathrm{dBm}$ und $1 \%$ Einstellung am Verstärker.

rigen Frequenzen der Amplitudenmodulation Probleme zu haben. Vor allem bei $10 \mathrm{~Hz}$ Modulationsfrequenz ist dies zu erkennen. Je höher diese Frequenz wird, desto sauberer wird das Rechtecksignal. Ein weiterer beobachteter Effekt ist auf den Verstärker zurückzuführen. Dieser liefert maximal $15 \mathrm{~W}$, die bei $0 \mathrm{dBm}$ Eingangsleistung erreicht werden. Bei höheren Eingangsleistungen kann der Verstärker dem anliegenden Signal nicht mehr folgen und schwächt dieses automatisch ab. Die Form des Signals könnte ebenfalls mit dem Regelkreis am Verstärkereingang zusammenhängen, der je nach anliegender Leistung diese reduziert oder eventuell bei zu langen Phasen sehr geringer Spannung am Eingang auch anhebt. Die Effekte, die nun in Kombination mit dem verstärkereigenen Regler, der hier auf $1 \%$ eingestellt war, auftreten, lassen sich nur schwer abschätzen. Aus diesem Grund 
wurden die bei den folgende Messungen vorgenommenen Einstellungen mit Hilfe dieser hier zum Teil gezeigten Messungen auf entsprechende Eingangsleistungen an $50 \Omega$ umgerechnet. Wurde in der Auswertung die mittlere Geschwindigkeit für Berechnungen genutzt, wurde auch die mittlere Leistung an $50 \Omega$ berechnet, indem die Spannung über die Dauer eines Modulationszyklus gemittelt wurde. In den Fällen, in denen die maximale Geschwindigkeit für die Berechnungen herangezogen wurde, wurde auch das Maximum der anliegenden Spannung für die Berechnung der Leistung benutzt.

\subsubsection{Gepulste Strömung in der Küvette}

Abbildung 7.12 zeigt eine Abfolge von Aufnahmen einer durch einen mit $10 \mathrm{~Hz}$ gepulsten Zwillings-Schallwandler erzeugten Strömung (siehe auch [108]). Hierbei wurde der Wandler mit $0 \mathrm{dBm}$ und $10 \%$ bei $1.82383 \mathrm{GHz}$ betrieben. Der Film wurde mit 2000 Bildern pro Sekunde aufgenommen, abgedruckt ist jedes zehnte Bild. Um die Strömung sichtbar zu machen, wurde eine kleine Menge Tinte direkt auf den Wandler in das DI-Wasser gegeben. Der erste Einschaltvorgang des Wandlers wurde aufgenommen.

Es ist zu erkennen, dass der erste Puls sich relativ langsam nach oben bewegt. Die Grenze zwischen Tinte und Wasser verläuft scharf und die nach oben schießende Tinte hat die Form eines Pilzes. Der zweite Puls ist wesentlich schneller als der erste und schießst auf etwa der Hälfte der Küvette durch den fast abgebremsten ersten Puls hindurch. Dieser wird dann von dem zweiten Puls mitgezogen. Der dritte Puls hat in etwa die gleiche Geschwindigkeit wie der zweite Puls. Außerdem ist zu erkennen, dass die Geschwindigkeit zu Beginn sehr viel höher ist und dann stark abnimmt. Die relativ kurze Reichweite des ersten Pulses könnte durch die höhere Viskosität der zu Beginn dicken Tintenschicht verursacht werden. Bei Erzeugen des zweiten Pulses hat die Menge der Tinte direkt über dem Wandler bereits abgenommen und Wasser und Tinte sind leicht vermischt, was eine Verringerung der Viskosität zur Folge hat.

Eine vergrößerte Darstellung einiger ausgewählter Bilder ist in Abbildung 7.13 gezeigt. Hier ist die innere Struktur der „Pilzköpfe“ durch den schnellen Start der Strömung zu erkennen. So bildet sich erst nahe der Wandleroberfläche ein recht kleiner Wirbel aus, der dann hochkatapultiert wird. Bei näherem Betrachten sind außerdem innerhalb des entstehenden Jets drei dunkler gefärbte Bereiche zu erkennen. So sind die beiden Ränder und die Mitte der Strömung intensiver gefärbt. Diese Struktur könnte durch die zwei einzelnen Wandler, die beide zu Beginn eine einzelne Strömung erzeugen, wie anhand des Array-Schallwandlers in Abbildung 7.4 bereits gezeigt wurde, hervorgerufen werden. 


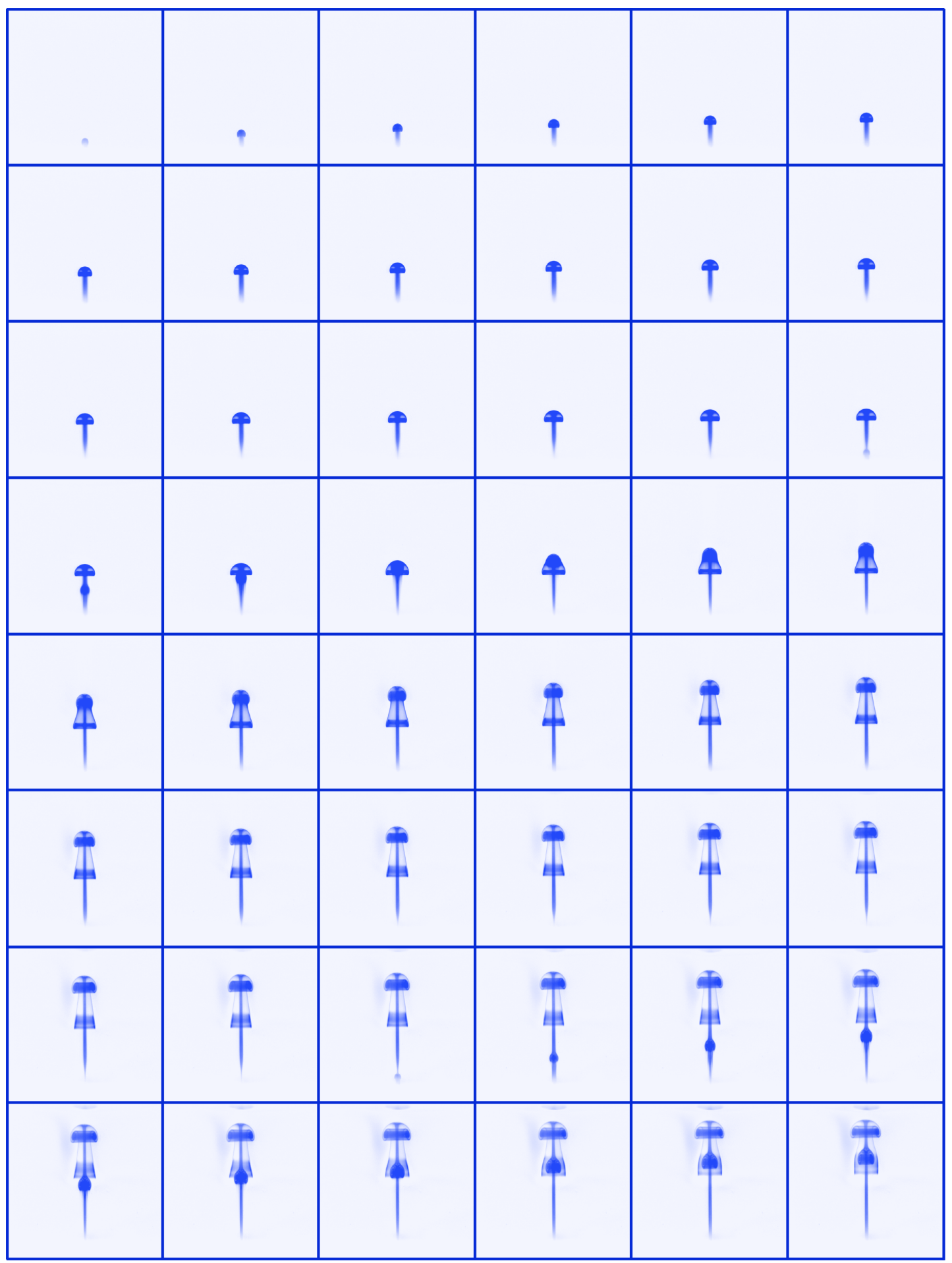

Abbildung 7.12.: Strömung über dem Zwillings-Schallwandler, sichtbar gemacht mit Tinte. Der Schallwandler wurde mit $0 \mathrm{dBm}$ und $10 \%$ bei $1.82383 \mathrm{GHz}$ betrieben. Der Film wurde mit 2000 Bps aufgenommen, abgedruckt ist jedes zehnte Bild. Die Breite eines Bildes beträgt etwa $1 \mathrm{~cm}$. Zur besseren Darstellung wurde das erste aufgenommene Bild ohne sichtbare Strömung als Hintergrund von allen Bildern abgezogen. Die Aufnahmen wurde nachträglich blau eingefärbt. 


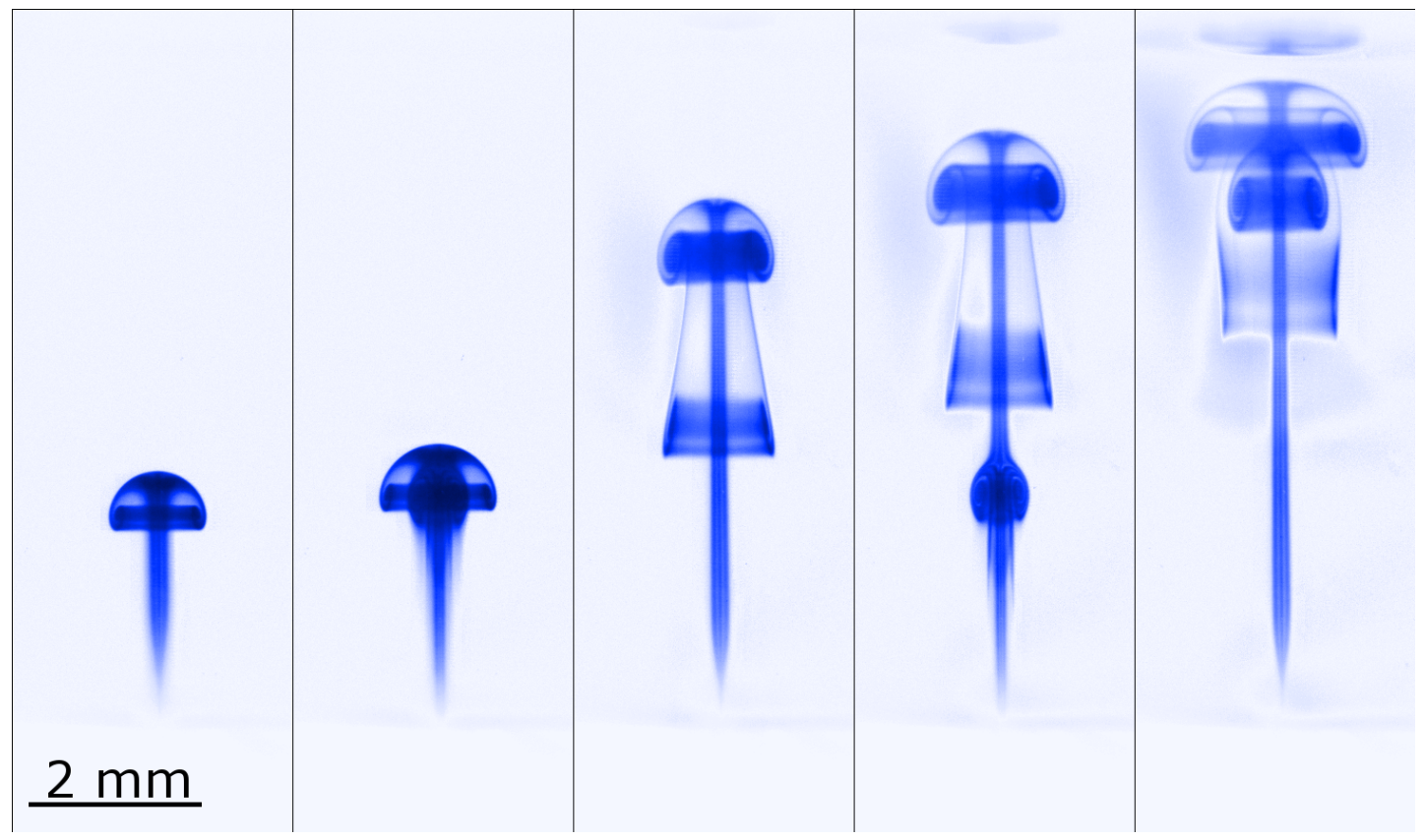

Abbildung 7.13.: Strömung über dem Zwillings-Schallwandler, sichtbar gemacht mit Tinte. Der Schallwandler wurde mit $0 \mathrm{dBm}$ und $10 \%$ bei $1.82383 \mathrm{GHz}$ betrieben. Der Film wurde mit 2000 Bps aufgenommen, abgedruckt ist eine willkürliche Folge von Bildern. Zur besseren Darstellung wurde das erste aufgenommene Bild ohne sichtbare Strömung als Hintergrund von allen Bildern abgezogen. Das Bild wurde nachträglich eingefärbt. Grafik ähnlich wie in [108].

In den Einzelbildern 1, 3 und 5 ist im unteren Bereich der Jetströmung das Ende des Einzelpulses zu erkennen. Die mitströmende Tinte wird schmaler und reift dann ab. Im vierten Einzelbild ist der dritte hochschießende Wirbel zu sehen. Außerdem sind zwei ,Schleppen“ links und rechts des eigentlichen Jets zu sehen, die, wie im füntten Teilbild erkennbar, durch die Rotation der Wirbel in diesen hineingezogen werden.

Um die Geschwindigkeit dieser hochschießenden Strömungs-Front zu ermitteln, wurde in jedem Bild des Filmes die Position der Tintenfront eines bestimmten Pulses bestimmt, daraus die Geschwindigkeit berechnet und über der Entfernung vom Wandler aufgetragen. Abbildung 7.14 zeigt die Ergebnisse für $0 \mathrm{dBm}$ und 10 und $20 \%$ der Verstärkerpotentiometereinstellung. Es ist zu erkennen, dass die maximale Geschwindigkeit nicht von der Verstärkerleistung abhängt. Bei 10\% Verstärkerpotentiometereinstellung (Teilabbildung 7.14(a)) werden vom ersten Puls etwa $24 \mathrm{~cm} \mathrm{~s}^{-1}$ erreicht. Durch die Tinte, die sich am Boden der Küvette befindet, kann der hochgeschossene Puls erst ab $1 \mathrm{~mm}$ Entfernung verfolgt wer- 


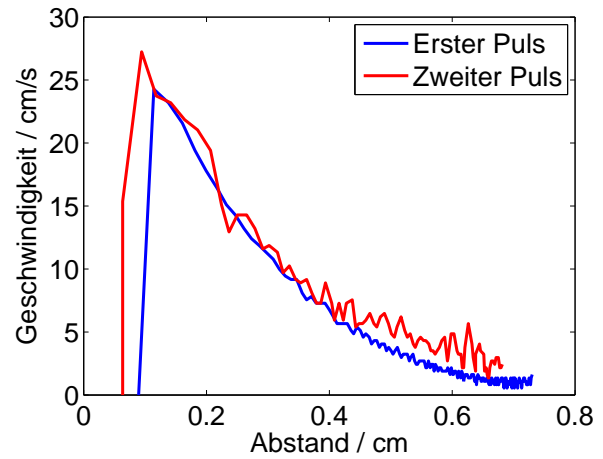

(a) $10 \%$ Verstärkerleistung.

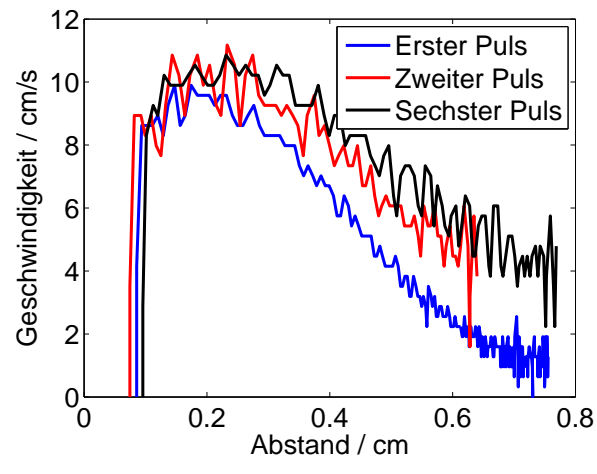

(b) $20 \%$ Verstärkerleistung.

\begin{abstract}
Abbildung 7.14.: Durch Tintenfront-Verfolgung ermittelte Geschwindigkeit in Abhängigkeit vom Abstand vom Zwillings-Schallwandler. Durch die den Wandler verdeckende Tinte ist die Geschwindigkeit erst nach etwa einem Millimeter zu bestimmen.
\end{abstract}

den. Der zweite Puls, der in die bereits strömende Flüssigkeit beschleunigt wird, erreicht in dieser Messung etwa $27 \mathrm{~cm} \mathrm{~s}^{-1}$. Des Weiteren ist zu erkennen, dass die Geschwindigkeit in weiterer Entfernung vom Wandler ebenfalls etwas höher ist als beim ersten Puls. Die höhere Schwankungsbreite des zweiten Pulses kommt durch die Tatsache zustande, dass bei diesem bereits Tinte in der Flüssigkeit vorhanden gewesen ist und sich somit die Front nicht so genau bestimmen lässt, wie beim ersten Puls.

In der zweiten Teilabbildung 7.14(b) für 20\% Verstärkerpotentiometereinstellung sind drei verschiedene Pulse (der erste, zweite und sechste) aufgetragen. Genau wie in der vorherigen Teilabbildung ist die Zunahme der Geschwindigkeit mit der Nummer des Pulses zu erkennen. Allerdings wird hier nur eine maximale Geschwindigkeit von $11 \mathrm{~cm} \mathrm{~s}^{-1}$ erreicht. Auch nimmt die Geschwindigkeit mit der Entfernung erst langsam zu und ist nicht nahe am Wandler am höchsten. Für diesen Effekt verantwortlich sind eventuell unterschiedliche Viskositäten der Tinte.

\title{
Zeitverlauf der Geschwindigkeit
}

Um die Abbildungen 7.15(a) und 7.15(b) zu erzeugen, wurde zu jedem Messzeitpunkt die maximale Geschwindigkeit ermittelt und über der Zeit aufgetragen. Im unmodulierten Betrieb in Teilabbildung 7.15(a) ist eine klare Zunahme der Geschwindigkeit mit der Leistung zu erkennen. Die gemessene maximale Geschwindigkeit weist starke Schwankungen auf. 
Die Messung bei $10 \mathrm{~Hz}$ Modulationsfrequenz zeigt einen Geschwindigkeitsverlauf, der der Form des elektrischen Signals in Abbildung 7.11(b) ähnelt. So nimmt die Geschwindigkeit erst langsam zu, weist einen Sprung auf und klingt dann langsam wieder ab. Allerdings ist keine Leistungsabhängigkeit der maximalen Geschwindigkeit zu erkennen. Die höchste Geschwindigkeit wird in dieser Messung bei der niedrigsten Leistung mit $38 \mathrm{~cm} \mathrm{~s}^{-1}$ erreicht.

Abbildung 7.16 zeigt die Zeitabhängigkeit der mittleren Geschwindigkeit, die durch Mittelung aller Geschwindigkeitsvektoren über ein immer gleiches Messfenster errechnet wurde. In Teilabbildung 7.16(a), für den unmodulierten Betrieb, ist wieder eine klare Abhängigkeit der Geschwindigkeit von der Leistung sichtbar. Die Schwankung der Messwerte ist hier wesentlich kleiner als in der Darstellung der Maximalwerte.

Im mit $10 \mathrm{~Hz}$ modulierten Betrieb (Teilabbildung 7.16(b)) ist eine leichte Abhängigkeit der maximal erreichten mittleren Geschwindigkeit von der Leistung zu erkennen. Bei der geringsten Leistung tritt auch die geringste maximale mittlere Geschwindigkeit auf. Die anderen beiden Leistungen liegen allerdings trotz recht großem Leistungsunterschied in der Geschwindigkeit recht nah beieinander. Die maximal erreichte mittlere Geschwindigkeit liegt bei $2.36 \mathrm{~cm} \mathrm{~s}^{-1}$.

\subsubsection{Untersuchung von Strömungen bei verschiedenen Lei- stungen}

\section{Spaltenweise Auftragung der gemessenen Geschwindigkeit}

Um zu überprüfen, ob die maximale Geschwindigkeit geeignet ist, um die Reynolds-Zahl und kinetische Energiedichte zu berechnen, wurde in jeder Spalte der PIV-Daten über die Dauer des gesamten aufgenommenen Films die maximale Geschwindigkeit gesucht und über der Position über dem Wandler aufgetragen (Abbildungen 7.17(a) und 7.17(b)). Die Form der erhaltenen Kurve legt nahe, dass es sich um tatsächlich gemessene Geschwindigkeiten und nicht um eventuelle Messfehler handelt. So tritt in der Mitte über dem Wandler ein deutliches Geschwindigkeitsmaximum auf, das zu den Rändern gaußförmig abfällt. In Teilabbildung 7.17(a) für den unmodulierten Betrieb ist eine klare Abhängigkeit von der am Schallwandler anliegenden elektrischen Leistung zu erkennen.

Im mit $10 \mathrm{~Hz}$ modulierten Betrieb (Teilabbildung 7.17(b)) fällt eine leichte Doppelspitze auf, die bei allen Leistungen zu beobachten ist, und die im Ansatz auch in Teilabbildung 7.17(a) zu sehen ist. Die Strömungsgeschwindigkeit ist im modulierten Betrieb unabhängig von der elektrischen Leistung. 


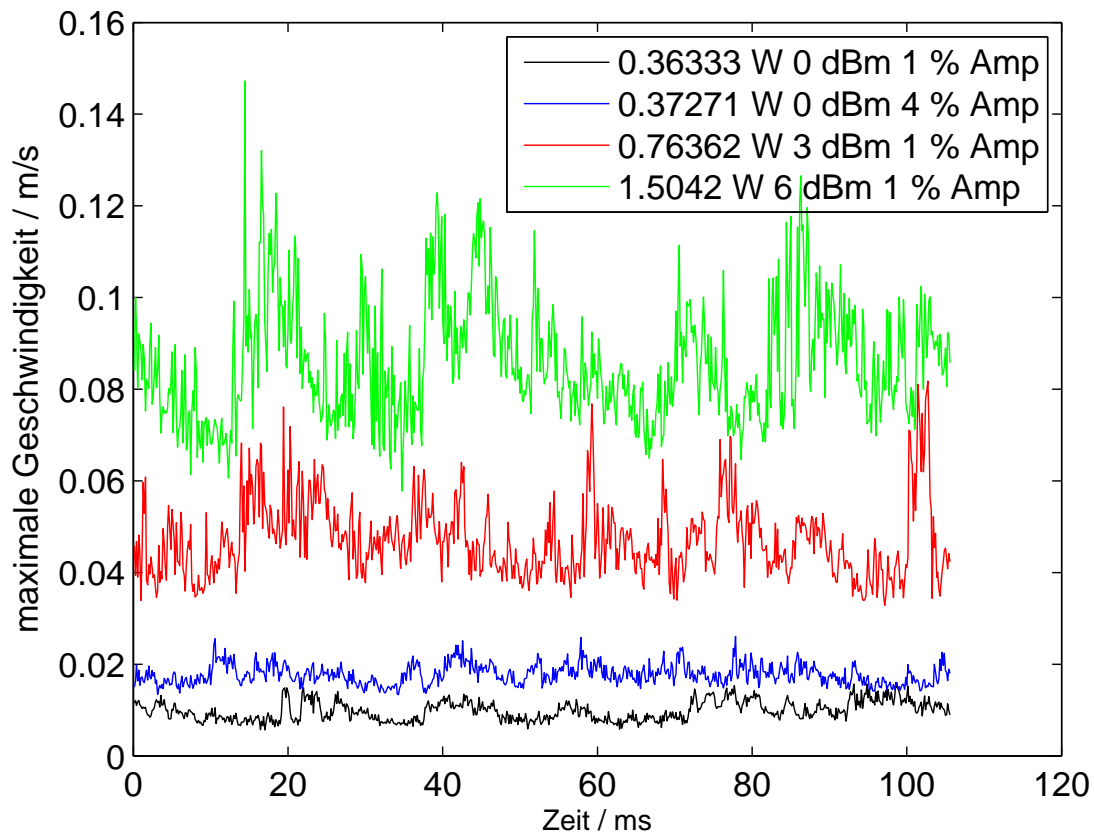

(a) Unmodulierter Betrieb.

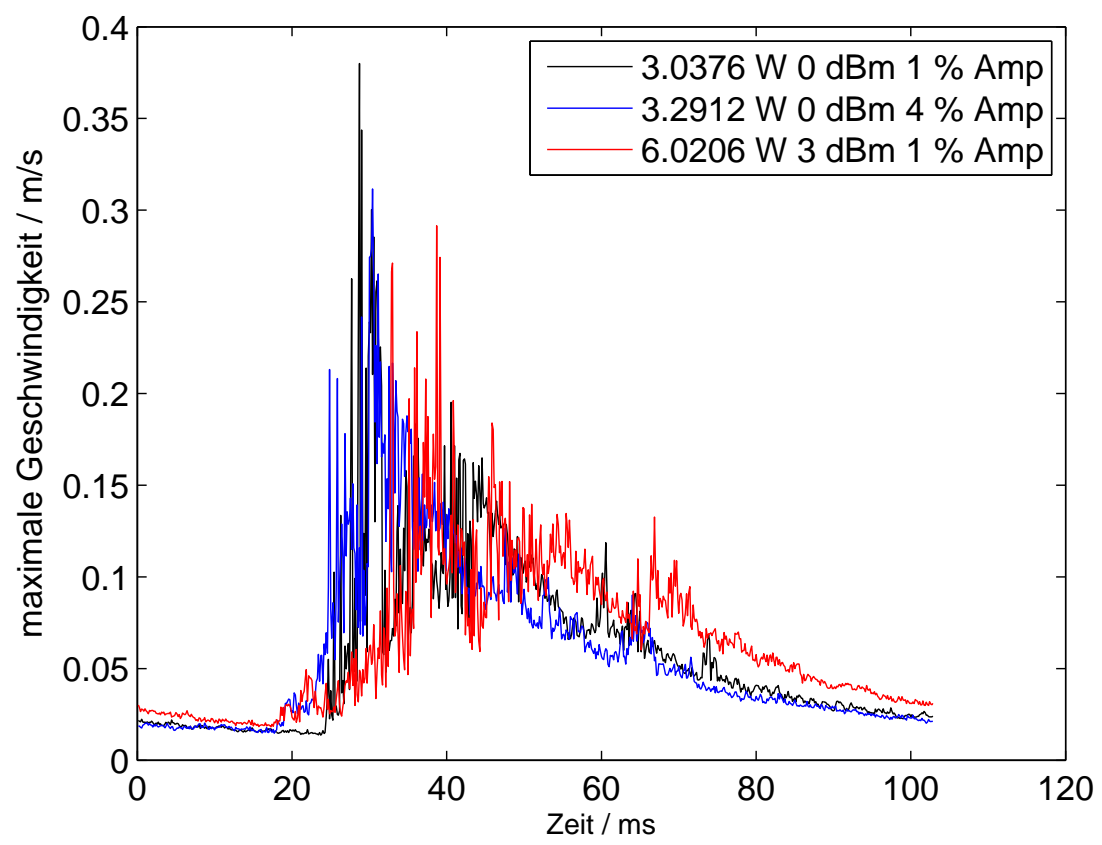

(b) Mit $10 \mathrm{~Hz}$ modulierter Betrieb.

Abbildung 7.15.: Zeitverlauf der maximalen Geschwindigkeit innerhalb des Strömungsfeldes am Zwillings-Schallwandler. Hinter der am Wandler anliegenden Leistung in der Legende sind die Einstellungen des Frequenzgenerators und des Potentiometers am Verstärkereingang angegeben. 


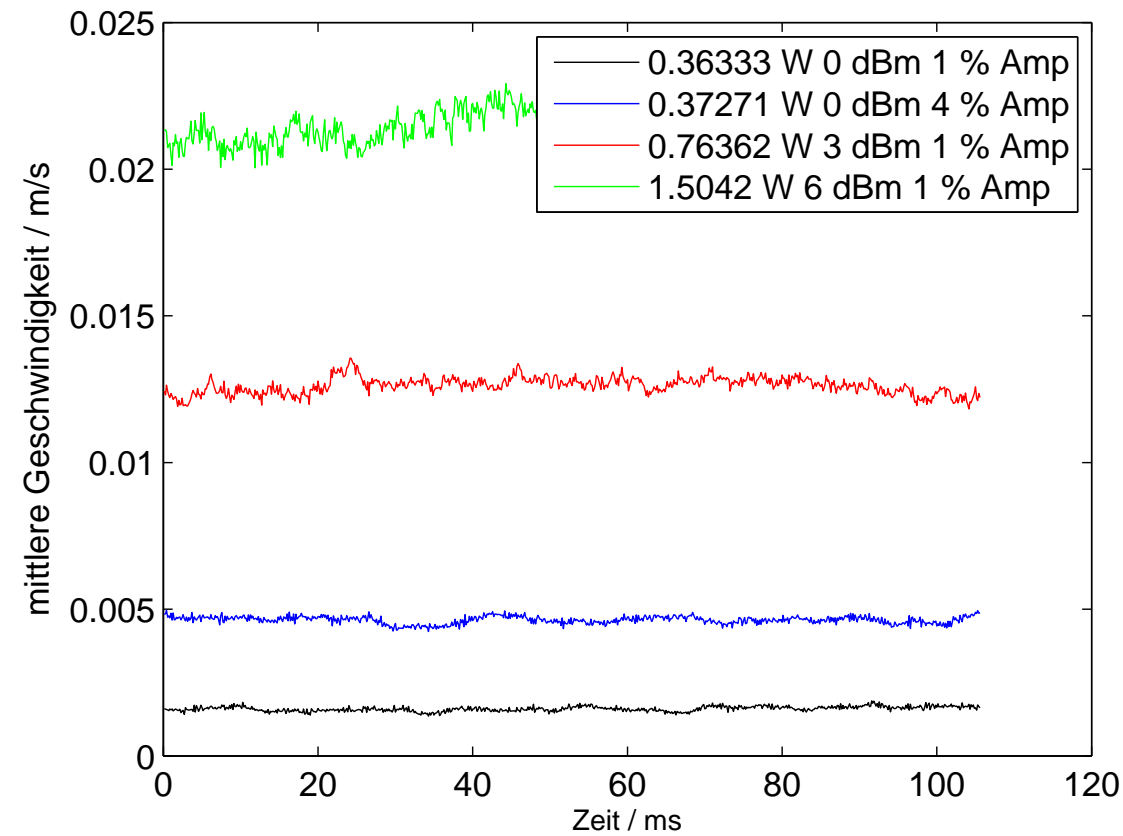

(a) Unmodulierter Betrieb.

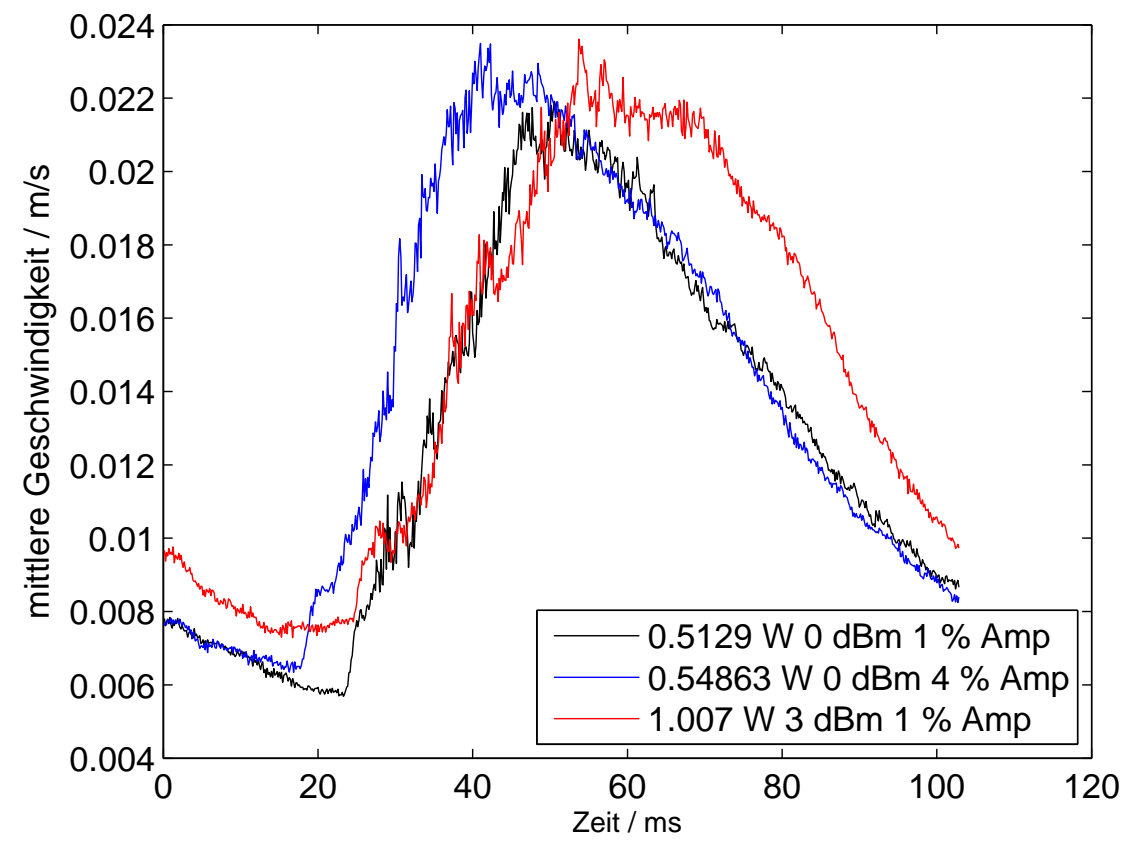

(b) Mit $10 \mathrm{~Hz}$ modulierter Betrieb.

Abbildung 7.16.: Zeitverlauf der mittleren Geschwindigkeit des Strömungsfeldes am Zwillings-Schallwandler. Hinter der am Wandler anliegenden Leistung in der Legende sind die Einstellungen des Frequenzgenerators und des Potentiometers am Verstärkereingang angegeben. 


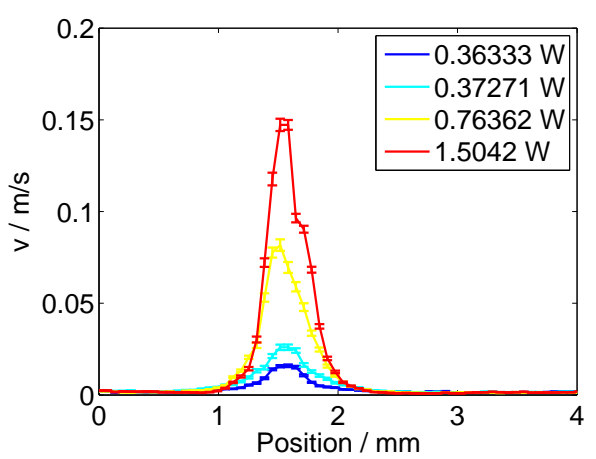

(a) Unmodulierter Betrieb.

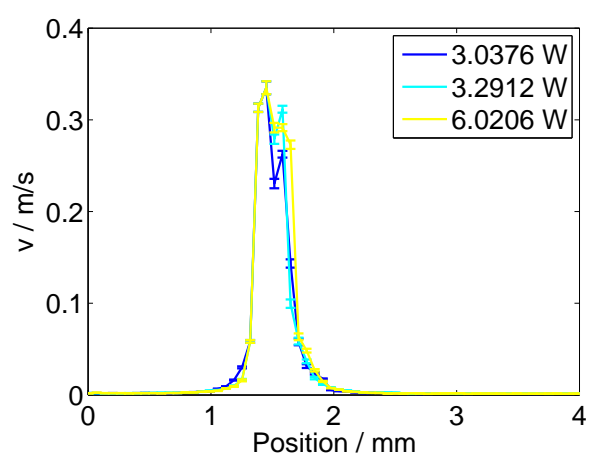

(b) Mit $10 \mathrm{~Hz}$ modulierter Betrieb.

Abbildung 7.17.: Spaltenweise Auftragung der Maximalen Geschwindigkeit des Strömungsfeldes am Zwillings-Schallwandler.

\section{Kinetische Energiedichte}

Um die kinetische Energiedichte zu ermitteln, wurden zum einen die mittlere Leistung und die mittlere kinetische Energiedichte über das gesamte Zeitfenster berechnet und zum anderen wurde die maximale Leistung wie in Kapitel 7.3.1 beschrieben und die maximale kinetische Energiedichte berechnet und aufgetragen. Die maximale kinetische Energiedichte berechnet sich hierbei über folgende Methode: Es wurde zu jedem Messzeitpunkt an jedem Punkt die kinetische Energiedichte berechnet. Für alle Messzeitpunkte wurde anschließend das jeweilige Maximum ermittelt und es wurden die lokalen Extrema der Zeitfunktion gesucht und nach Größe absteigend geordnet. Aus den ersten 20 Werten wurde dann die mittlere maximale kinetische Energiedichte berechnet. Die ersten 20 Werte wurden gewählt, weil sich im Fall des unmodulierten Betriebs mit dieser Anzahl die gleichen Werte ergaben, wie mit der gesamten mittleren Energiedichte. Aus diesem Grund sind die Messwerte des unmodulierten Betriebs in Abbildung 7.18 nur einmal zu erkennen. Es ist zu sehen, dass die Messwerte im unmodulierten Betrieb einen recht linearen Verlauf zeigen. Die Messwerte der mittleren kinetischen Energiedichte für den modulierten Betrieb liegen alle etwas höher und zeigen im Mittel ebenfalls einen mit der Leistung ansteigenden Verlauf. Die Messwerte der mittleren maximalen kinetischen Energiedichte steigen zwar auch etwas an, liegen aber sehr dicht beieinander und zeigen keinen monotonen Anstieg mit der Leistung. Insgesamt liegen die Werte der mittleren maximalen kinetischen Energiedichte im gepulsten Betrieb sehr viel höher als die Werte der mittleren kinetischen Energiedichte, was durch die Beschränkung auf die höchsten Werte während des Pulses und die höhere Ausgangsleistung des Verstärkers im modulierten Betrieb zu erwarten war. 


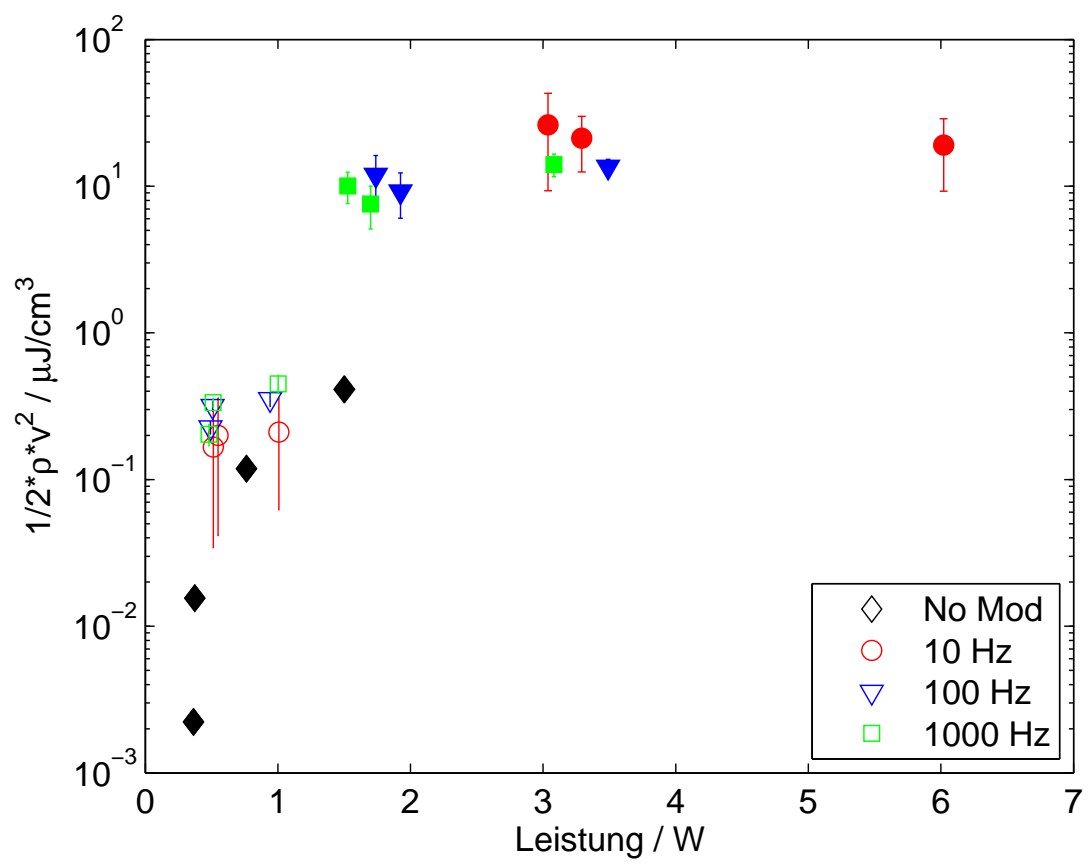

\begin{abstract}
Abbildung 7.18.: Kinetische Energiedichte am Zwillings-Schallwandler aufgetragen über der vom Verstärker zur Verfügung gestellten Energie. Ungefüllte Punkte sind für gemittelte Geschwindigkeiten und gemittelte Energie pro Pulsperiode und gefüllte Punkte für maximale Geschwindigkeit und maximale kurzzeitig zur Verfügung gestellte Energie eingetragen. Die Messwerte für den unmodulierten Betrieb sind in beiden Fällen identisch und deshalb nur einmal zu erkennen.
\end{abstract}

\title{
Reynolds-Zahl
}

Für die Auftragung der Reynolds-Zahl wurde jeweils die maximal gemessene Geschwindigkeit über den gesamten Messzeitraum gesucht, daraus (wie in Kapitel 4.7.2) die Reynolds-Zahl berechnet, und über der aus der maximal gemessenen Spannung berechneten Leistung (siehe Kapitel 7.3.1) aufgetragen (Abbildung 7.19). Die Reynolds-Zahl steigt bei niedrigen Leistungen und unmoduliertem Betrieb homogen bis etwa 190 an. Bei moduliertem Betrieb schwanken die Werte relativ stark, nehmen aber in der Tendenz auch zu. Die maximale ReynoldsZahl von etwa 410 wird bei $10 \mathrm{~Hz}$ Modulationsfrequenz erreicht und ist in dieser Messung unabhängig von der anliegenden Leistung. Auch bei den anderen beiden Modulationsfrequenzen lässt sich kein von der Leistung abhängender Verlauf feststellen. 


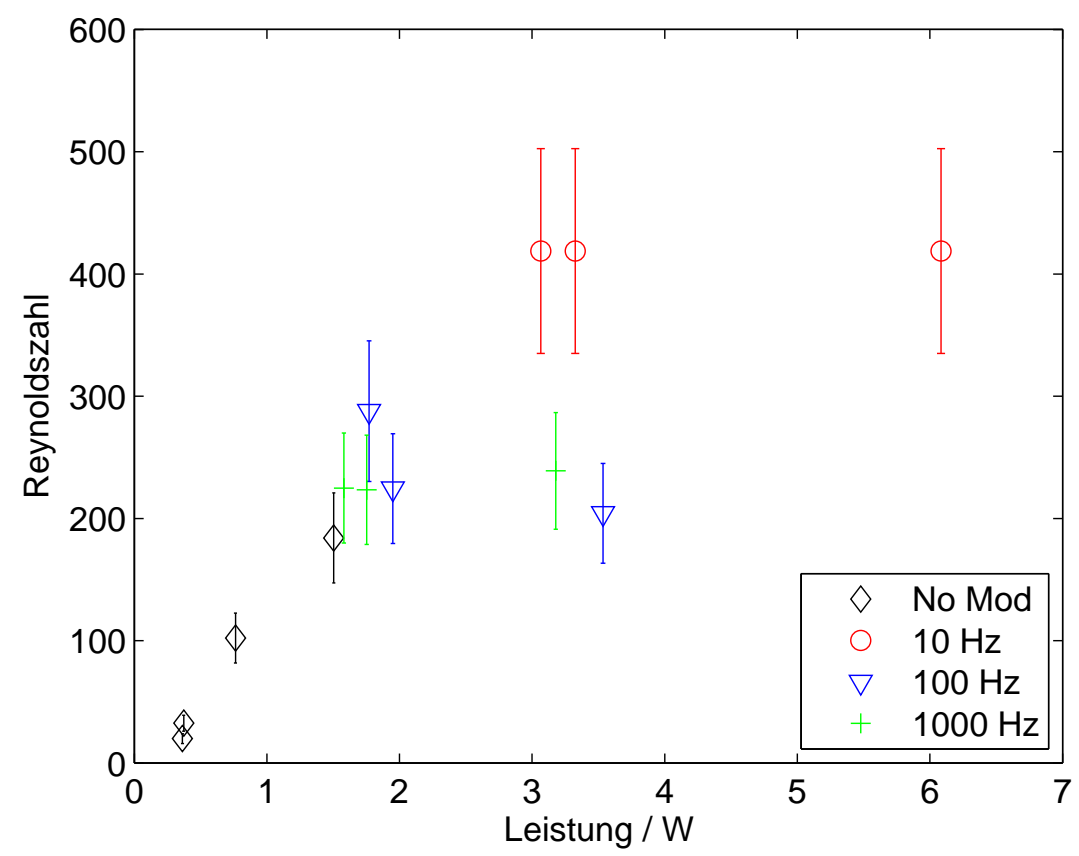

Abbildung 7.19.: Reynolds-Zahl der Strömung am ZwillingsSchallwandler aufgetragen über der vom Verstärker zur Verfügung gestellten Energie.

\subsubsection{Elektrochemische Messung}

Abbildungen 7.20(a) und 7.20(b) zeigen Elektrochemiemessungen der Strömung über dem Zwillings-Schallwandler. Bei der Messung in $y$-Richtung in Teilabbildung 7.20(a) sind drei Strömungsspitzen zu erkennen. Außerdem sind in einem Abstand von etwa $400 \mu \mathrm{m}$ zwei Minima des gemessenen Stromes zu sehen. Außerhalb nimmt die Stromstärke wieder etwas zu. Die Struktur mit den drei Maxima in der Mitte ist eventuell durch die Größe der Messelektrode zu erklären. Diese ist etwas breiter als die Breite der aktiven Wandlerfläche und beeinflusst je nach Position die Richtung und Stärke der Wirbel zwischen Wandleroberfläche und der durch die Elektrode gebildeten Grenzfläche. Allerdings lassen sich auch in den Aufnahmen des Strömungsfeldes mit Tinte (siehe Kapitel 7.3.2) drei dunklere Bereiche ausmachen, was auf eben diese drei Maxima zurückzuführen wäre. Die Messung in der anderen Richtung (Teilabbildung 7.20(b)), parallel zu den elliptischen Wandlerflächen, zeigt nur ein Maximum und zwei Minima, die einen Abstand von etwas über $200 \mu \mathrm{m}$ haben. Genau wie in der vorherigen Abbildung folgen auf die Minima Regionen mit einem etwas höheren gemessenen Strom. 


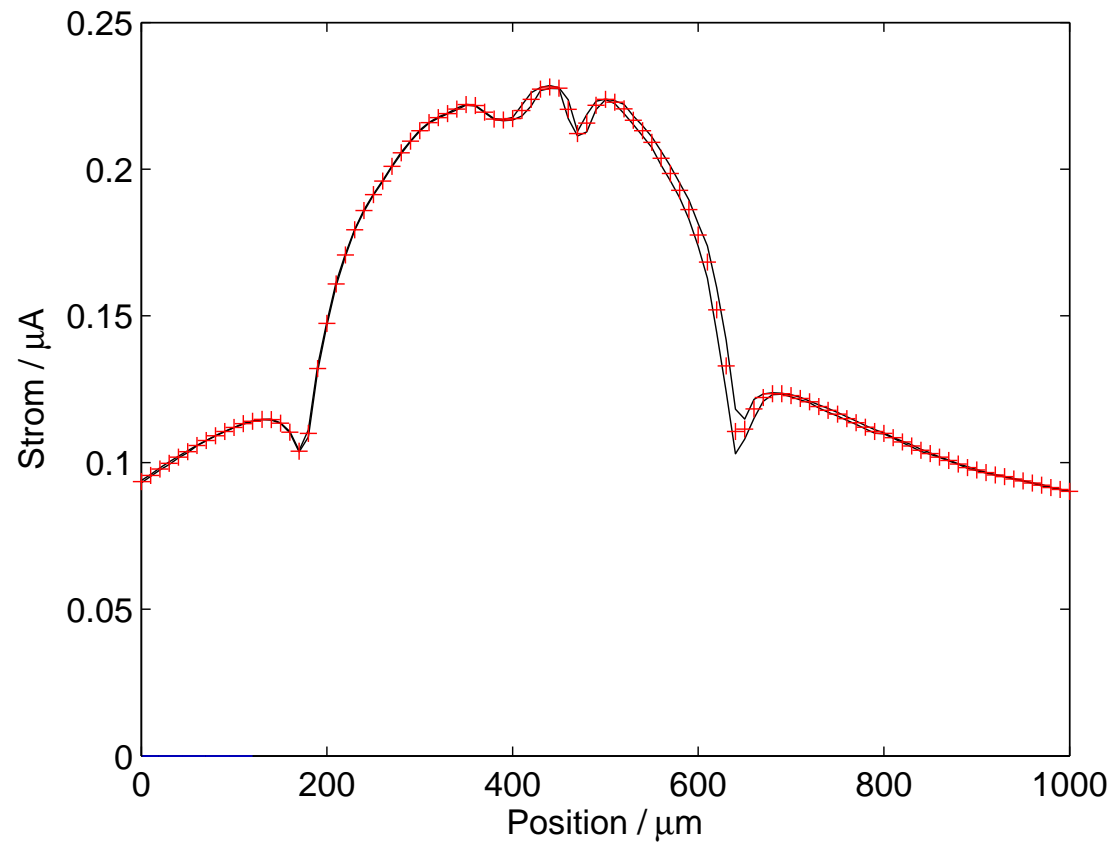

(a) Position in $y$-Richtung, senkrecht zur Blickrichtung der Kamera aus den PIVAufnahmen.

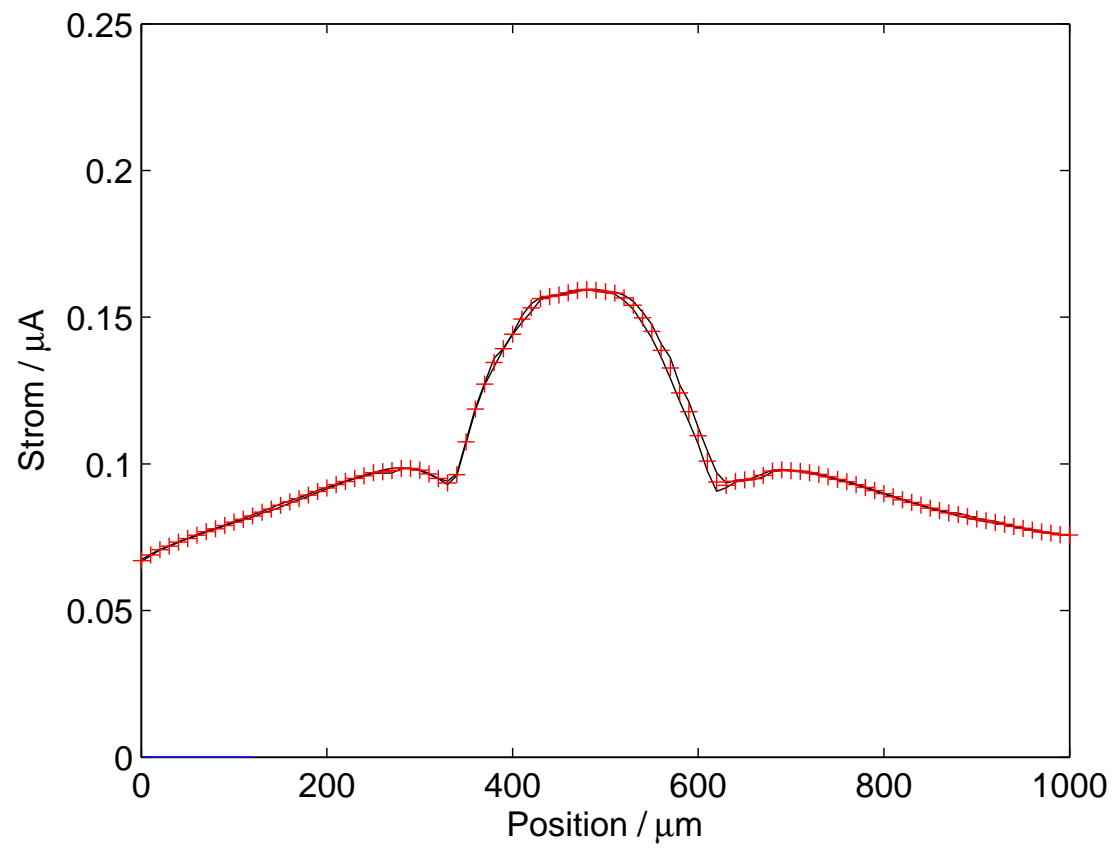

(b) Position in $x$-Richtung, parallel zur Blickrichtung der Kamera aus den PIVAufnahmen.

Abbildung 7.20.: Messungen des Stromes in Abhängigkeit von der Position über dem Zwillings-Schallwandler. Die Elektrode wurde in einer Entfernung von $100 \mu \mathrm{m}$ bewegt. 


\section{Kapitel 8 FEM-Simulation von akustischen Strömungen im Gigahertzbereich}

Um die durch die Gigahertz-Schallwandler eingebrachte Volumenkraft abschätzen zu können, wurden FEM-Simulationen mit COMSOL [44] durchgeführt. Es wurden akustische Simulationen unter Einbeziehung der kompletten Schallwandler, akustische Simulationen mit einer schwingenden Grenzfläche und vereinfachte Volumenkraftsimulationen durchgeführt.

\subsection{Akustische Simulationen mit Comsol}

\subsubsection{Schallfelder und Strömungen bei $400 \mathrm{MHz}$}

Um die Effekte des gesamten Schallwandlers bestehend aus Elektroden, Piezokeramik, Anpassungsschichten und Trägermaterial abschätzen zu können, wurden Simulationen bei 400 und $800 \mathrm{MHz}$ Anregungsfrequenz durchgeführt. Aufgrund der besseren Sichtbarkeit der Effekte werden hier nur die Ergebnisse der $400 \mathrm{MHz}$ Simulation gezeigt. Diese niedrigeren Frequenzen wurden gewählt, weil durch die kurze Wellenlänge bei $2 \mathrm{GHz}$ ein sehr feines Gitter für die Berechnungen benötigt wird, was bei der Größe der Struktur zu sehr langen Rechenzeiten führen würde. Die Wellenlängen waren also um einen Faktor 5 größer, was aber zu ähnlichen Effekten führt wie die experimentelle Frequenz $2 \mathrm{GHz}$.

Für die Simulationen wurde ein rotationssymmetrisches Modell mit Piezoelektrik, Schallausbreitung im Festkörper und in Flüssigkeiten gewählt (AcousticPiezoelectric Interaction). Für diese Simulation wurde ein kombiniertes Gitter, bestehend aus Rechtecken im unteren Bereich und Dreiecken im oberen Bereich aus insgesamt 588328 Elementen verwendet. Die maximale Gittergröße beträgt $1 \times 10^{-5} \mathrm{~m}$, beziehungsweise im Bereich des Wasser ein Fünftel der Wellenlänge. Außerdem wurden weitere Verfeinerungen im Bereich des Piezomaterials, der Elektroden und der Impedanzanpassungsschicht vorgenommen. Die Rechenzeit 
betrug knapp drei Minuten. Die Ergebnisse dieser Simulation wurden genutzt, um die Größe der durch die Schallabsorption resultierenden Volumenkraft in der Flüssigkeit zu berechnen, die dann zu einer gleichgerichteten Strömung führt. Für die Simulation der Strömung wurde in diesem Fall ein Modell für Creeping Flow benutzt, das für kleine Reynolds-Zahlen gültig ist. Auch hier wurde ein aus Rechtecken im unteren Bereich und Dreiecken im oberen Bereich kombiniertes Gitter verwendet. Die maximale Größe der Elemente beträgt $1 \times 10^{-6} \mathrm{~m} \mathrm{im}$ unteren und $1 \times 10^{-5} \mathrm{~m}$ im oberen Bereich. Das gesamte Gitter besteht aus 125881 Elementen und die Rechenzeit betrug etwa fünf Minuten. Der gesamte Wandler besteht aus zwei Goldelektroden zum Anlegen der Spannung, die das Piezomaterial umschließen. Dann folgt das Wafermaterial, auf das noch eine Impedanzanpassungsschicht aufgebracht ist, um die Schallabstrahlung in das Wasser zu verbessern. Die Dicken der Materialien und andere verwendete Parameter sind in Tabelle 8.1 angegeben. An die Elektroden wird eine Spannung von $11 \mathrm{~V}$ mit einer Frequenz von $400 \mathrm{MHz}$ angelegt.

Tabelle 8.1.: Die verwendeten Parameter für die piezoelektrische Simulation bei $400 \mathrm{MHz}$

\begin{tabular}{|r|l|}
\hline Elektrodendicke & $<1 \mu \mathrm{m}$ \\
\hline Piezomaterialdicke & $>1 \mu \mathrm{m}$ \\
\hline Piezodurchmesser & $200 \mu \mathrm{m}$ \\
\hline Impedanzanpassungsschicht & $<1 \mu \mathrm{m}$ \\
\hline Waferdicke & $500 \mu \mathrm{m}$ \\
\hline Wasserhöhe & $500 \mu \mathrm{m}$ \\
\hline Frequenz $f$ & $400 \mathrm{kHz}$ \\
\hline Dämpfungskoeffizient $\alpha$ & $25.3 * f^{2} * 10^{-15} \mathrm{~m}^{-1} \mathrm{~Hz}^{-2}[109]$ \\
\hline Spannung & $11 \mathrm{~V}$ \\
\hline
\end{tabular}

Abbildung 8.1 zeigt die darauf folgende Auslenkung des Wandlers und den resultierenden Schalldruck im Wasser. Die maximale Auslenkung innerhalb des Schallwandlers beträgt $0.87 \mathrm{~nm}$. An der Oberfläche werden etwa $0.7 \mathrm{~nm}$ erreicht. Der daraus resultierende maximale Schalldruck beträgt direkt an der Oberfläche des Wandlers bis $2.88 \times 10^{6} \mathrm{~Pa}$, der maximale Unterdruck beträgt $-3.08 \times 10^{6} \mathrm{~Pa}$. Wie zu erkennen ist, wird der Hauptteil des Schalls auf der Achse des Piezos abgestrahlt und geht kaum über die Breite des Piezomaterials hinaus. Seitlich sind nur sehr viel schwächere Auslenkungen zu sehen. 


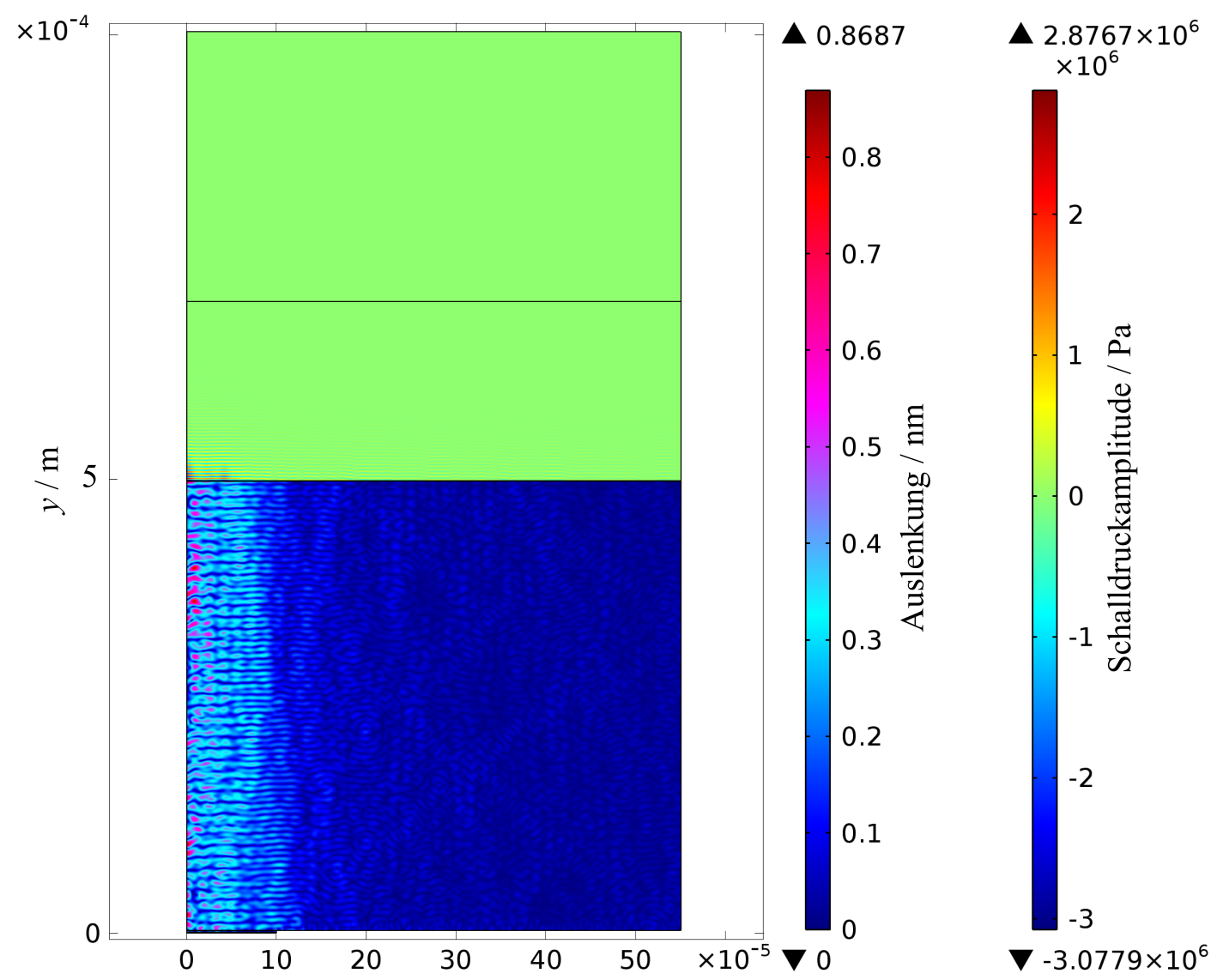

Abbildung 8.1.: Rotationssymmetrische Schallfeldsimulation eines $200 \mu \mathrm{m}$ breiten Schallwandlers (die Symmetrieachse befindet sich auf der linken Seite) bei $400 \mathrm{MHz}$ und $11 \mathrm{~V}$ anliegender Spannung am Piezo. Der Druck ist in der Einheit $\mathrm{Pa}$, die Auslenkung in nm angegeben. Der Piezo befindet sich unten links. Aufbau von unten nach oben: Elektrode Au $(<1 \mu \mathrm{m})$, Piezomaterial $\mathrm{ZnO}(>1 \mu \mathrm{m})$, Elektrode $\mathrm{Au}(<1 \mu \mathrm{m})$, Wafer $\mathrm{Si}$ (500 $\mu \mathrm{m})$, Impedanzanpassungsschicht $\mathrm{SiO}_{2}(<1 \mu \mathrm{m}), \mathrm{H}_{2} \mathrm{O}(500 \mu \mathrm{m})$.

Einen vergrößerten Ausschnitt des resultierenden Schallfelds in Wasser zeigt Abbildung 8.2. Es ist zu erkennen, dass trotz des langen Wegs des Schalls durch den Wafer, auch das resultierende Schallfeld relativ konzentriert über dem Piezo abgestrahlt wird. Die Wellenberge und Wellentäler der abgestrahlten Schallwelle und die Wellenlänge sind ebenfalls deutlich sichtbar. Innerhalb von etwa $70 \mu \mathrm{m}$ ist bei der verwendeten Farbskala kein Schalldruck mehr zu sehen.

Ein vergrößerter Ausschnitt eines kleinen Teils des Wafers, der Impedanzanpassungsschicht und des Wassers zeigt Abbildung 8.3. Die Skala der Auslenkung im Wafer und in der Impedanzanpassungsschicht ist die gleiche wie in der vorherigen 


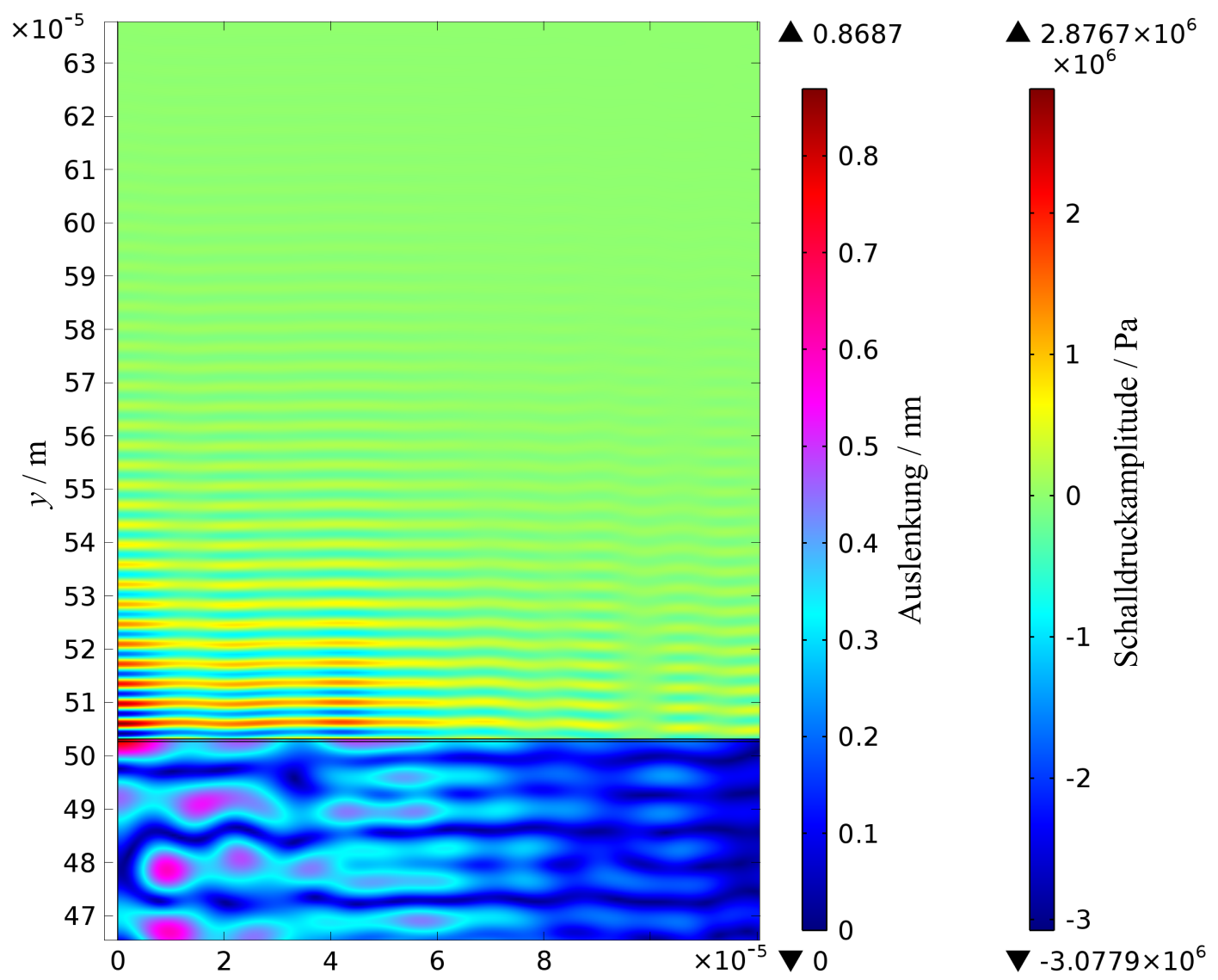

Abbildung 8.2.: Rotationssymmetrische Schallfeldsimulation eines $200 \mu \mathrm{m}$ breiten Schallwandlers (die Symmetrieachse befindet sich auf der linken Seite) bei $400 \mathrm{MHz}$ und $11 \mathrm{~V}$ anliegender Spannung am Piezo. Der Druck ist in der Einheit $\mathrm{Pa}$, die totale Auslenkung in $\mathrm{nm}$ angegeben.

Abbildung. Im oberen Teil der Abbildung (Wasser) ist die wirksame Volumenkraft gezeigt. Es wird ein Maximalwert von $9.07 \times 10^{9} \mathrm{~N} \mathrm{~m}^{-3}$ erreicht, der aber nur in einem sehr kleinen Bereich direkt über und in der Mitte des Wandlers auftritt. Mit zunehmendem Abstand vom Schallwandler klingt die Volumenkraft sehr schnell ab, obwohl die Reichweite des Schallfelds sehr viel weiter ist.

Das aus der piezoelektrischen Simulation resultierende Strömungsfeld ist in Abbildung $8.4 \mathrm{zu}$ sehen. Trotz des sich erst durch den Wafer ausbreitenden Schalls und des etwas verbreiterten Schallfelds im Wasser ist die Hauptströmung im Strömungsfeld auf die Mitte über dem Wandler konzentriert. Hier strömt es mit einer maximalen Geschwindigkeit von etwa $63 \mathrm{~cm} \mathrm{~s}^{-1}$ vom Wandler weg nach oben. Durch die oben geschlossene Struktur wird die Strömung oben zur Seite umgelenkt und bildet einen Wirbel, dessen Zentrum leicht rechts von der maximalen Strömung liegt. Eine weitere Auffälligkeit zeigt sich direkt an der Wand- 


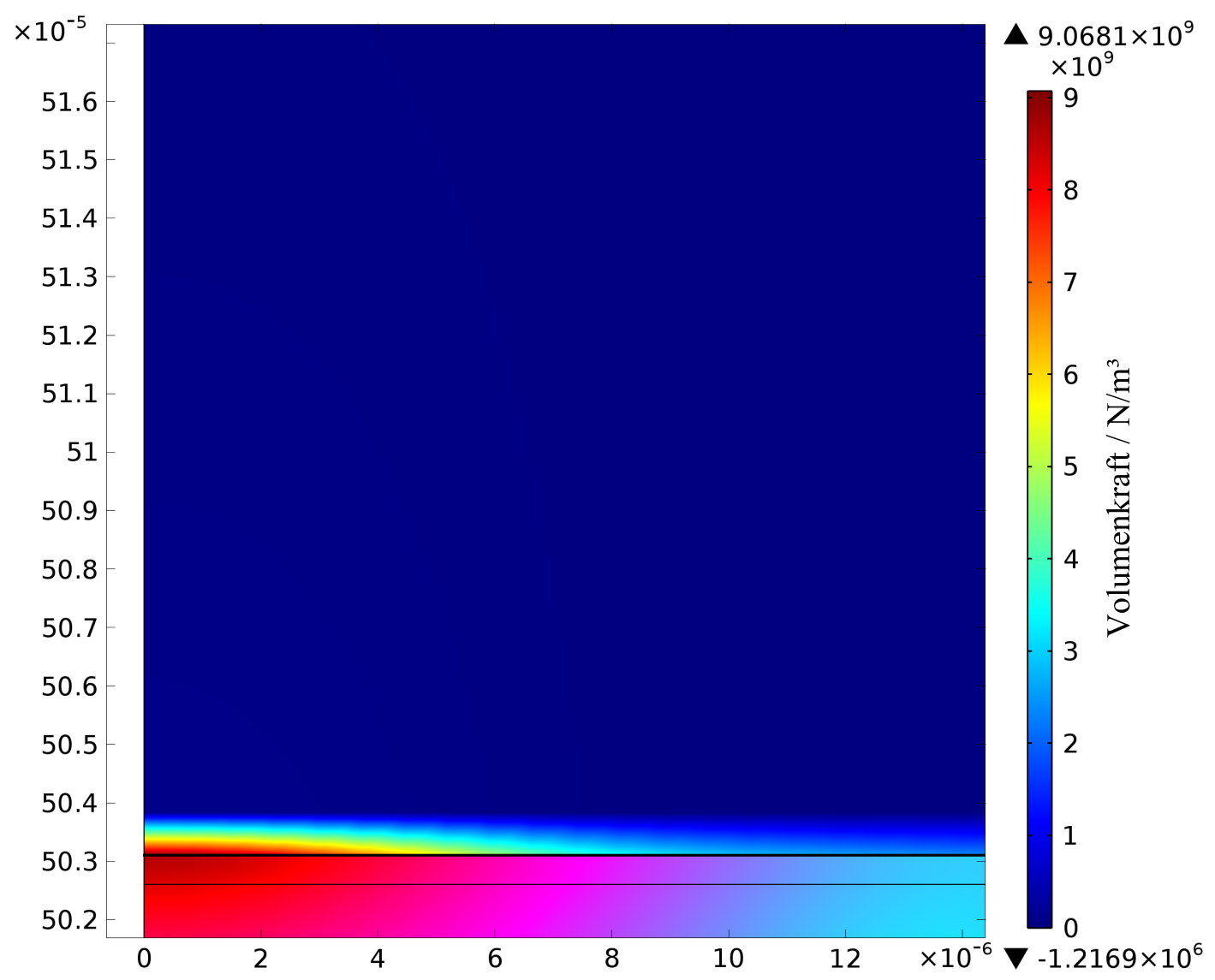

Abbildung 8.3.: Rotationssymmetrische Schallfeldsimulation eines $200 \mu \mathrm{m}$ breiten Schallwandlers (die Symmetrieachse befindet sich auf der linken Seite) bei $400 \mathrm{MHz}$ und $11 \mathrm{~V}$ anliegender Spannung am Piezo. Die Volumenkraft ist in der Einheit $\mathrm{N} \mathrm{m}^{-3}$ angegeben.

leroberfläche. So sind rechts von der Position des Piezos Wirbel mit teilweisen Rückströmungen direkt an der Wandleroberfläche zu erkennen. Dies könnte an der komplexen Struktur des Schallfelds im Wafer und somit auch der komplexen Struktur des Schallfelds im Wasser liegen.

\subsubsection{Schallfelder und Strömungen bei $2 \mathrm{GHz}$}

Die Simulationen bei $2 \mathrm{GHz}$ wurde ohne den Aufbau des Schallwandlers, nur mit einer beschleunigten Grenze an einer Seite der Flüssigkeitsdomäne mit dem Acoustics-Modul von Comsol mit den Unterpunkten Pressure Acoustics und Creeping Flow durchgeführt. Durch die sehr kleinen Wellenlängen bei diesen Frequenzen benötigt eine vollständige Simulation zu viele Gitterelemente und damit eine $\mathrm{zu}$ hohe Rechenzeit. Auch in dieser reduzierten Variante musste eine ma- 


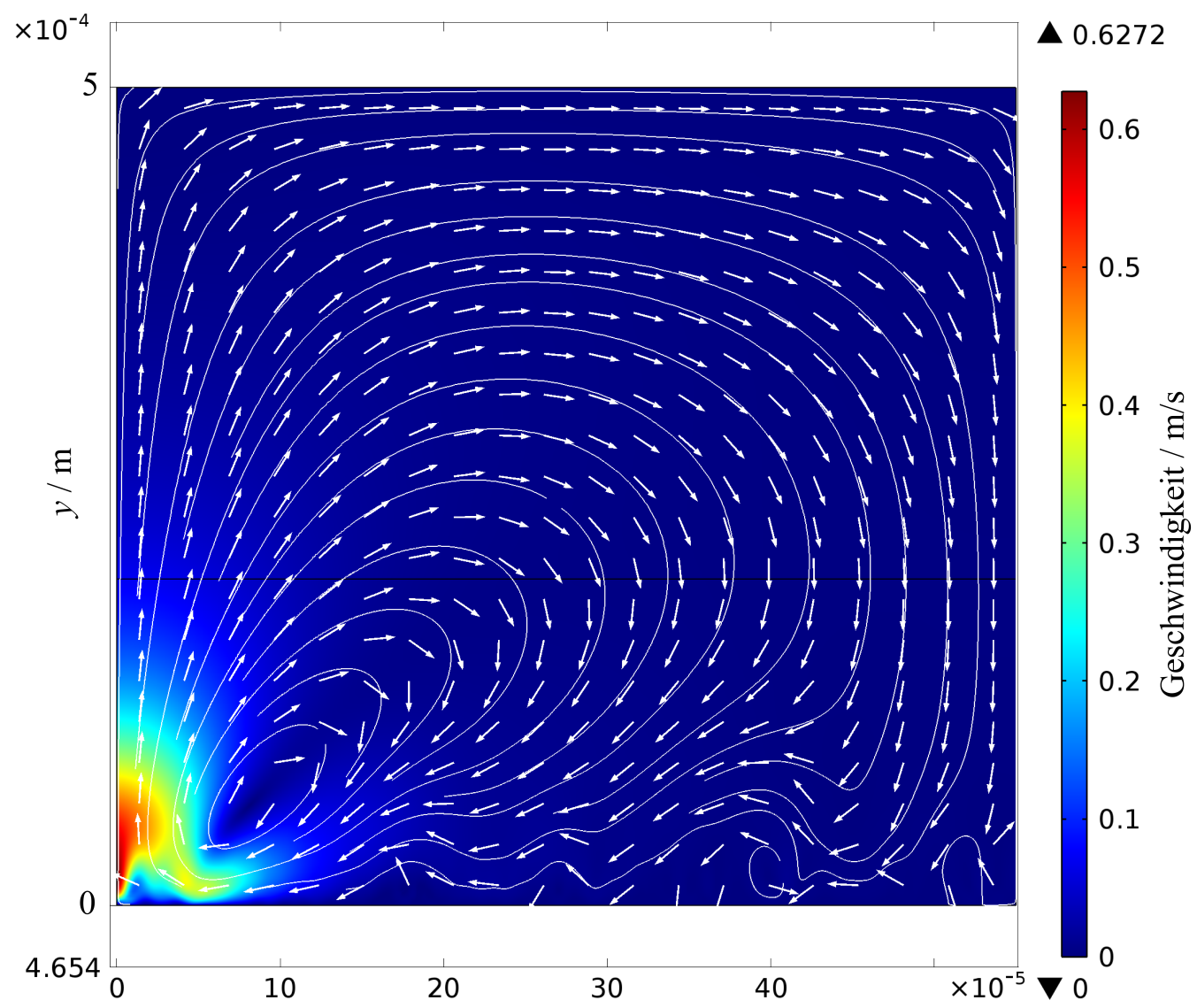

Abbildung 8.4.: Strömung eines $200 \mu \mathrm{m}$ breiten Schallwandlers (die Symmetrieachse befindet sich auf der linken Seite) bei $400 \mathrm{MHz}$ und $11 \mathrm{~V}$ anliegender Spannung am Piezo. Die Strömung ist in $\mathrm{m} \mathrm{s}^{-1}$ angegeben.

ximale Elementgröße von $1 \times 10^{-6} \mathrm{~m}$ verwendet werden. Die minimale Elementgröße beträgt $1 \times 10^{-8} \mathrm{~m}$, außerdem wurde im unteren Bereich ein noch feineres Grenzflächengitter verwendet. Das gesamte Gitter besteht aus 4787046 Elementen. Die Rechenzeit betrug etwa drei Stunden. Es handelt sich also um eine stark vereinfachte akustische Simulation, bei der ausschließlich das Schallfeld in der Flüssigkeit simuliert wurde. Dieses Schallfeld wurde für die Berechnung einer Volumenkraft genutzt. Die daraus resultierende Strömung wurde auch in diesem Fall durch eine Creeping Flow Simulation berechnet. Abbildung 8.5 zeigt das resultierende Schallfeld eines $150 \mu \mathrm{m}$ breiten Wandlers mit $0.1 \mathrm{~nm}$ Auslenkung. Dieses Schallfeld ist zu den Seiten sehr scharf begrenzt und wird innerhalb von etwa $40 \mu \mathrm{m}$ fast vollständig absorbiert.

Um das Schallfeld etwas genauer betrachten zu können, ist in Abbildung 8.6 ein vergrößerter Ausschnitt abgebildet. Es ist zu erkennen, dass am Rand des Wandlers eine Zylinderwellenabstrahlung (hier aufgrund der Zweidimensionalität eine 


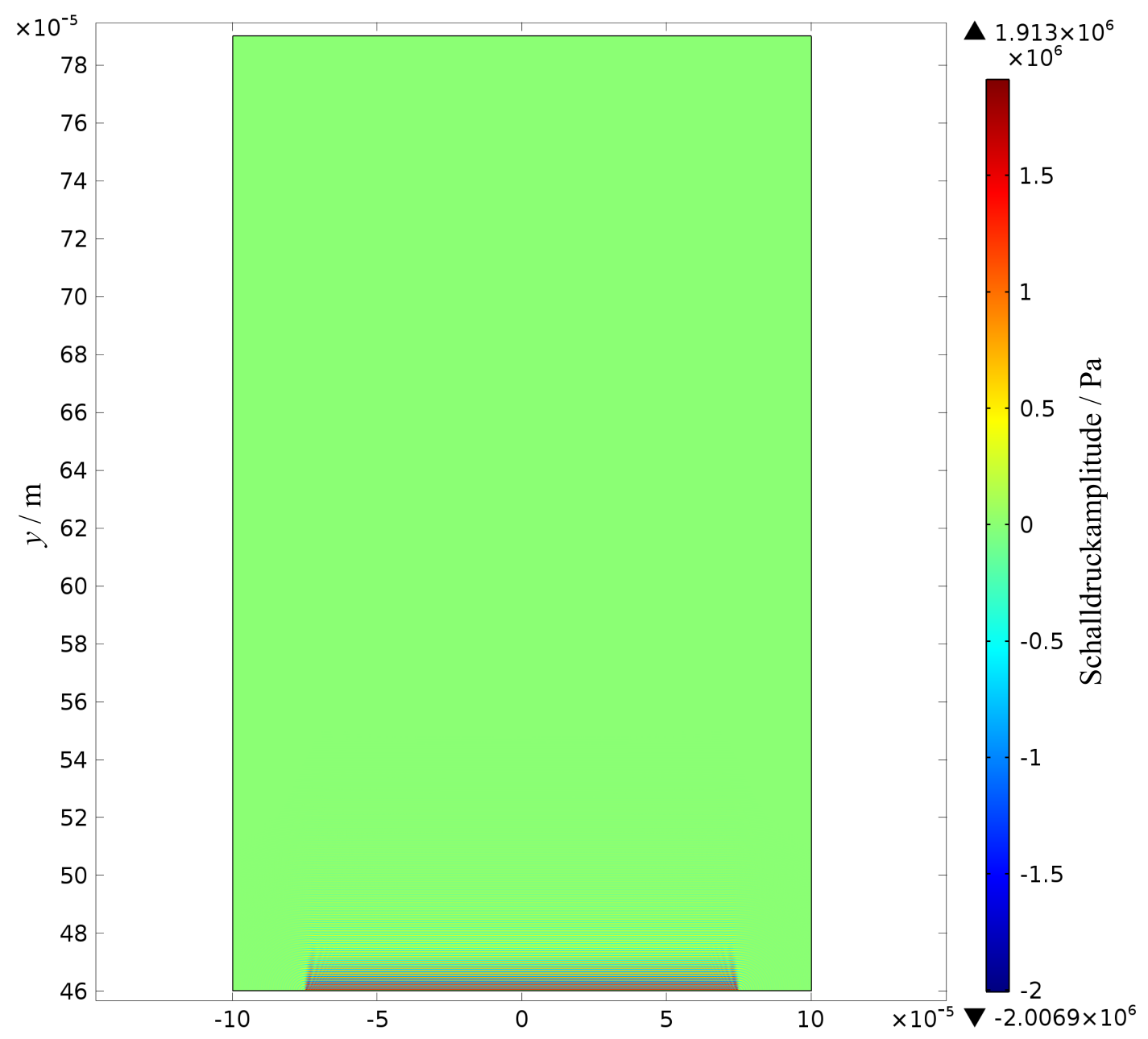

Abbildung 8.5.: 2D-Schallfeldsimulation eines $150 \mu \mathrm{m}$ breiten Schallwandlers bei $1.83 \mathrm{GHz}$ und $0.1 \mathrm{~nm}$ Auslenkung. Der Druck ist in der Einheit $\mathrm{Pa}$ angegeben.

Kreiswelle) stattfindet. Daran schließen sich leichte Bündelungseffekte und Interferenzen an. Richtung Wandlermitte werden die Wellen zunehmend ungestört und lassen sich als ebene Wellen behandeln.

Abbildung 8.7 zeigt die aus dem oben abgebildeten Schallfeld resultierende Volumenkraft. Der Rahmen verdeutlicht den in Abbildung 8.8 in der vergrößerten Ansicht gezeigten Bereich. In der Übersicht ist die Volumenkraft kaum zu erkennen, nur bei sehr genauem Hinsehen fällt direkt über dem Wandler ein kleiner Bereich wirkender Volumenkraft auf. In der vergrößerten Ansicht ist die Struktur der Volumenkraft im Randbereich des Wandlers abgebildet. Die Interferenzen in diesem Bereich rufen eine starke Volumenkraft hervor. In Richtung Wandlermitte nimmt die Interferenzstruktur zunehmend ab und es ist nur noch ein sehr kleiner 


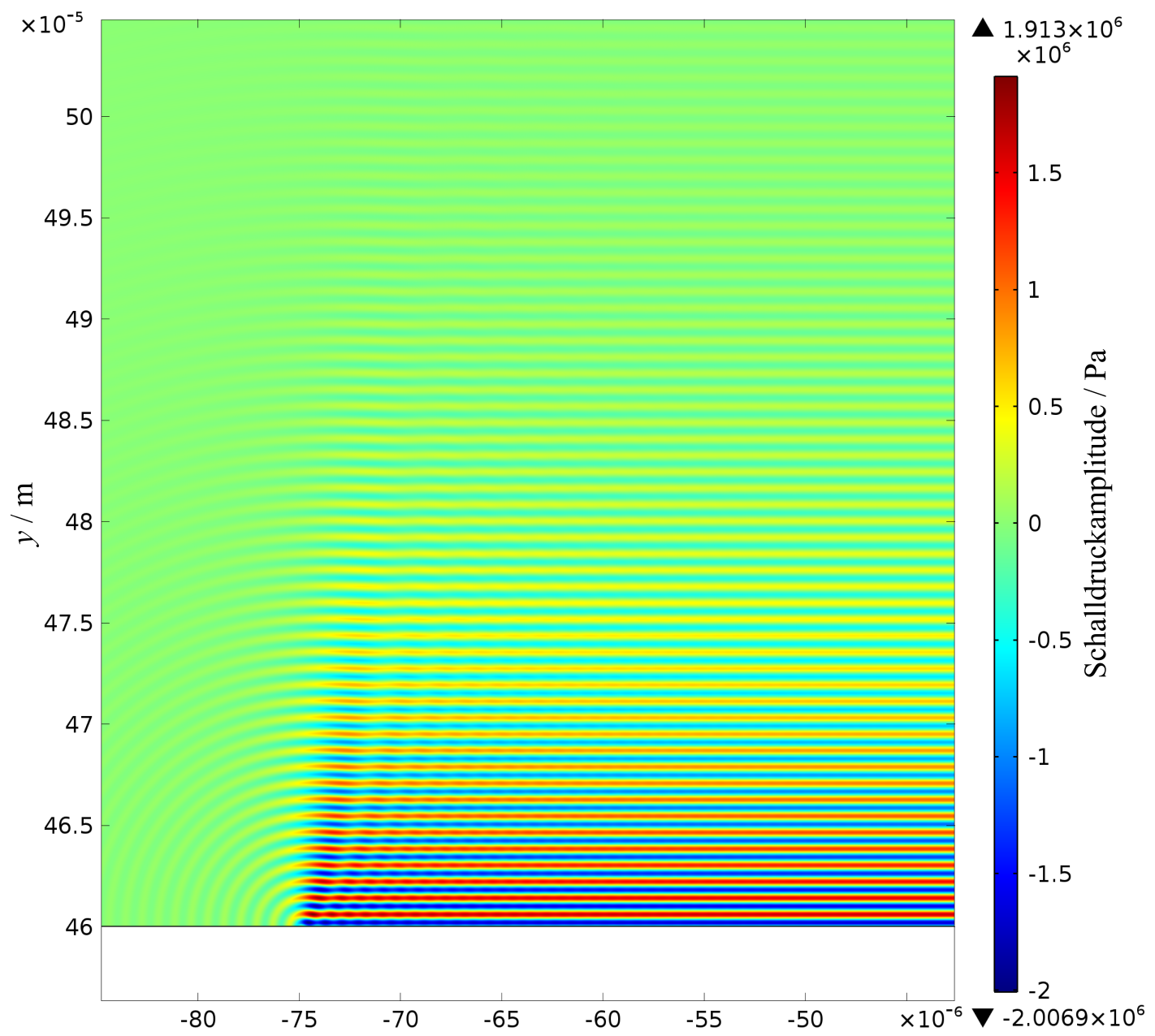

Abbildung 8.6.: Randbereich der 2D-Schallfeldsimulation eines $150 \mu \mathrm{m}$ breiten Schallwandlers bei $1.83 \mathrm{GHz}$ und $0.1 \mathrm{~nm}$ Auslenkung. Der Druck ist in der Einheit Pa angegeben.

Bereich hoher Volumenkraft direkt an der Wandleroberfläche zu erkennen. Trotz der komplexen Struktur der Volumenkraft folgt im Zeitmittel das in Abbildung 8.9 abgebildete Strömungsfeld. Da es sich um eine 2D-Simulation handelt, ergeben sich im Vergleich zu einer dreidimensionalen Strömung für die Zuströmungen an den Rändern des Wandlers zu hohe Werte. Bei Rotationssymmetrie nimmt die Strömungsgeschwindigkeit bei konstantem Volumenstrom durch die größere Entfernung (und somit Gewichtung) von der Rotationsachse ab. Unter Berücksichtigung dieser Gewichtung wird die maximale Strömungsgeschwindigkeit also mit etwa $30 \mathrm{~cm} \mathrm{~s}^{-1}$ direkt über dem Wandler erreicht. Diese Geschwindigkeit tritt in einer Entfernung von etwa 60 bis etwa 120 pm auf. Darüber beträgt die Geschwindigkeit ungefähr $20 \mathrm{~cm} \mathrm{~s}^{-1}$. 


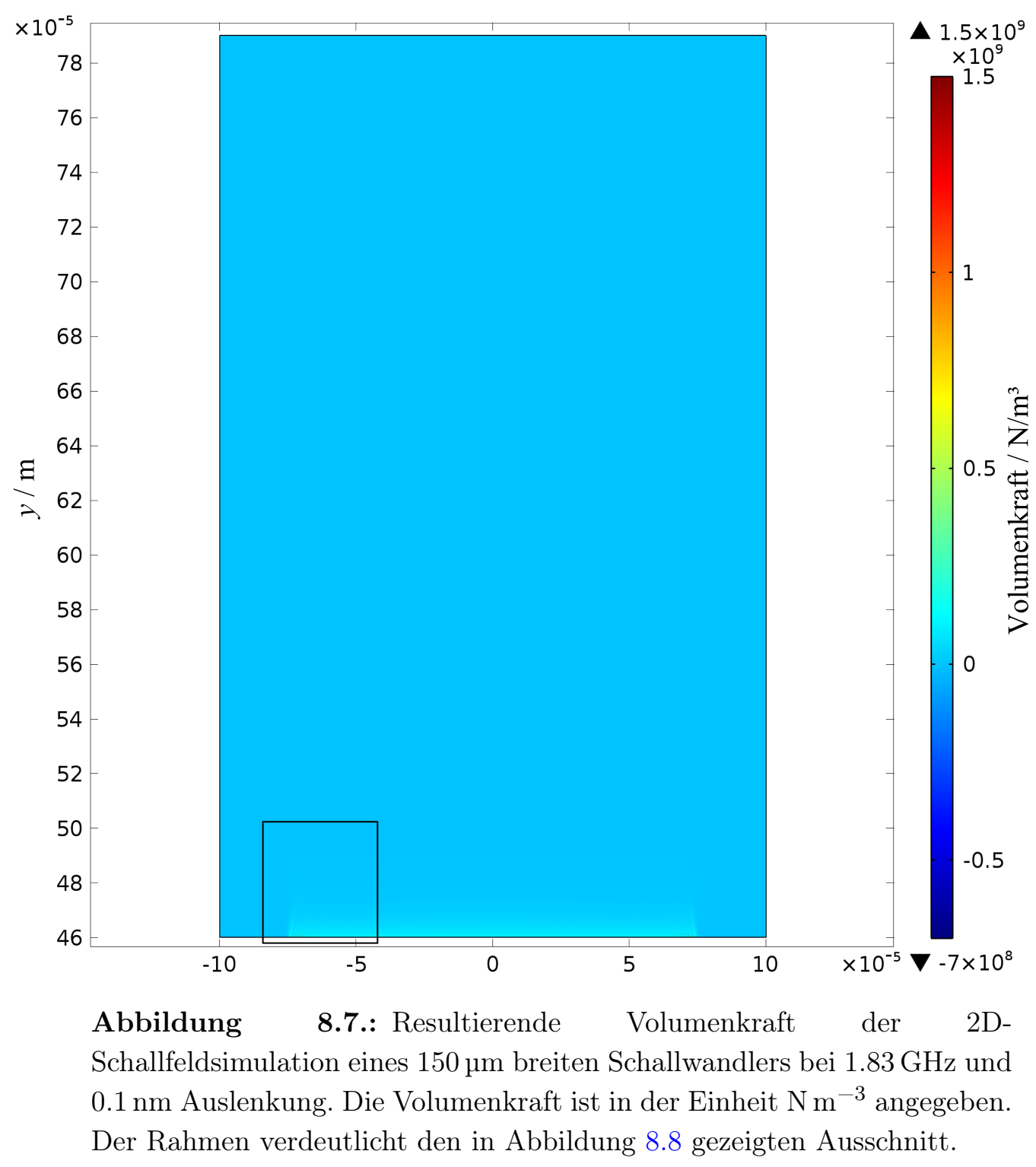

\subsection{Volumenkraftsimulationen mit Comsol}

Aufgrund der Ergebnisse der akustischen Simulationen wurden die großräumigen Simulationen von Array-Schallwandler und Zwillings-Schallwandler und vollständiger Küvettenströmung mit einer reinen Volumenkraftsimulation in 2D durchgeführt. Hierzu wurde in COMSOL in den Bereichen der Wandler eine nach Formel (2.16) mit der Entfernung von der Oberfläche exponentiell abklingende auf die Flüssigkeit nach oben wirkende Volumenkraft der Form $F_{0} \mathrm{e}^{-2 \alpha y}$ angelegt. Zur Simulation der Strömung wurde eine Laminar Flow Simulation verwendet. 


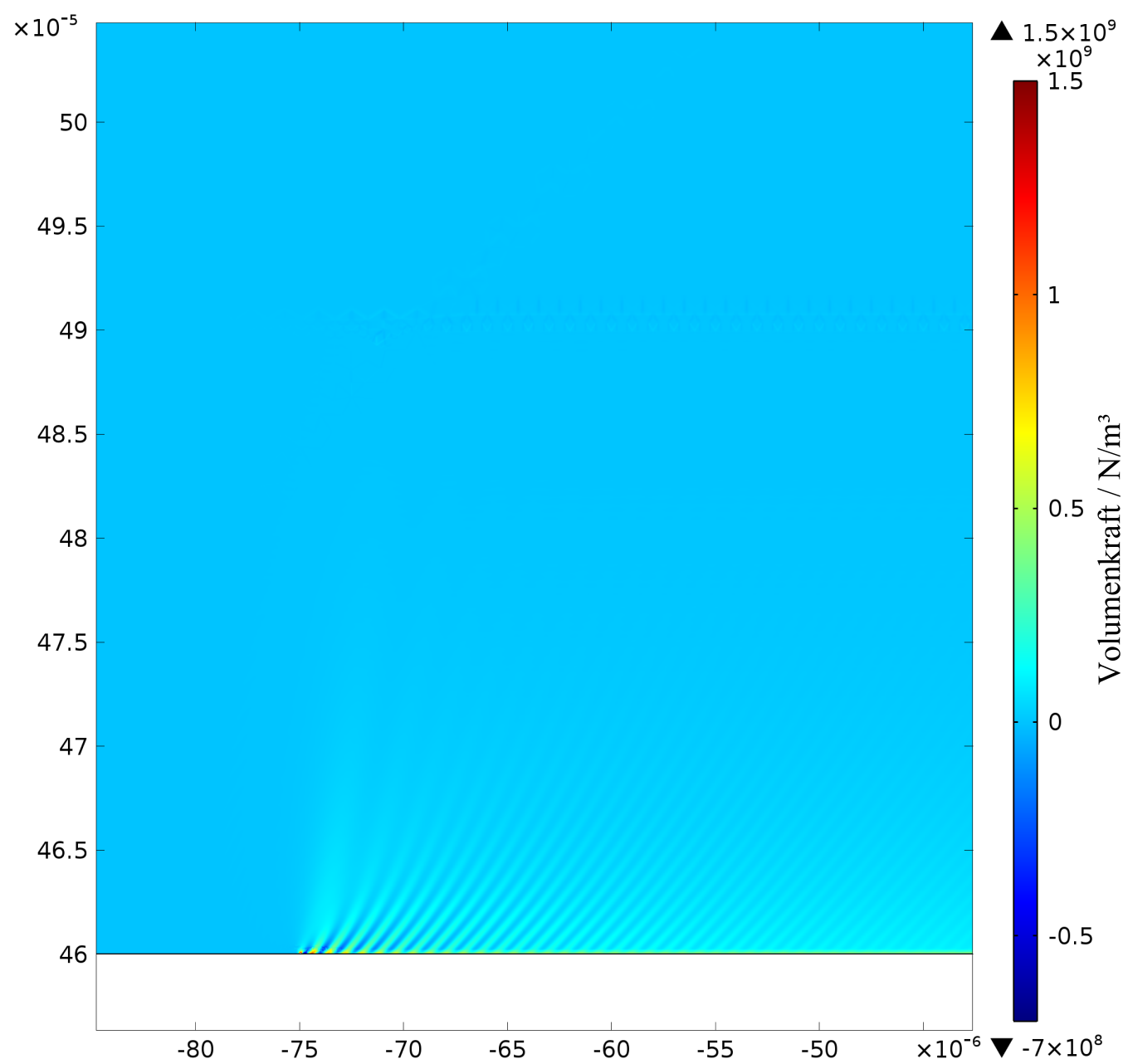

Abbildung 8.8.: Randbereich der Volumenkraft der 2DSchallfeldsimulation eines $150 \mu \mathrm{m}$ breiten Schallwandlers bei $1.83 \mathrm{GHz}$ und $0.1 \mathrm{~nm}$ Auslenkung. Die Volumenkraft ist in der Einheit $\mathrm{Nm}^{-3}$ angegeben. Für eine bessere Sichtbarkeit wurde der Wertebereich der Volumenkraft begrenzt. Die eigentlichen Grenzen sind $-1.21 \times 10^{10}$ und $2.12 \times 10^{10} \mathrm{~N} \mathrm{~m}^{-3}$.

\subsubsection{Array-Schallwandler}

Für die Simulation der Array-Schallwandler wurde ein Gitter mit insgesamt 433308 Einzelelementen verwendet. Die minimale Elementgröße beträgt $2 \times 10^{-6}$, die maximale Elementgröße $6.7 \times 10^{-5} \mathrm{~m}$. Die Rechenzeit betrug knapp sechs Minuten. Mit 15 einzelnen Wandlern mit einer Breite von je $150 \mu \mathrm{m}$ und einem Abstand von $50 \mu \mathrm{m}$ ergibt sich das in Abbildung 8.10 abgebildete Strömungsfeld. Die Strömungen der einzelnen Wandler schließen sich zu einer großräumigen Strömung zusammen, die die gesamte simulierte Küvette ausfüllt. Durch die anströ- 


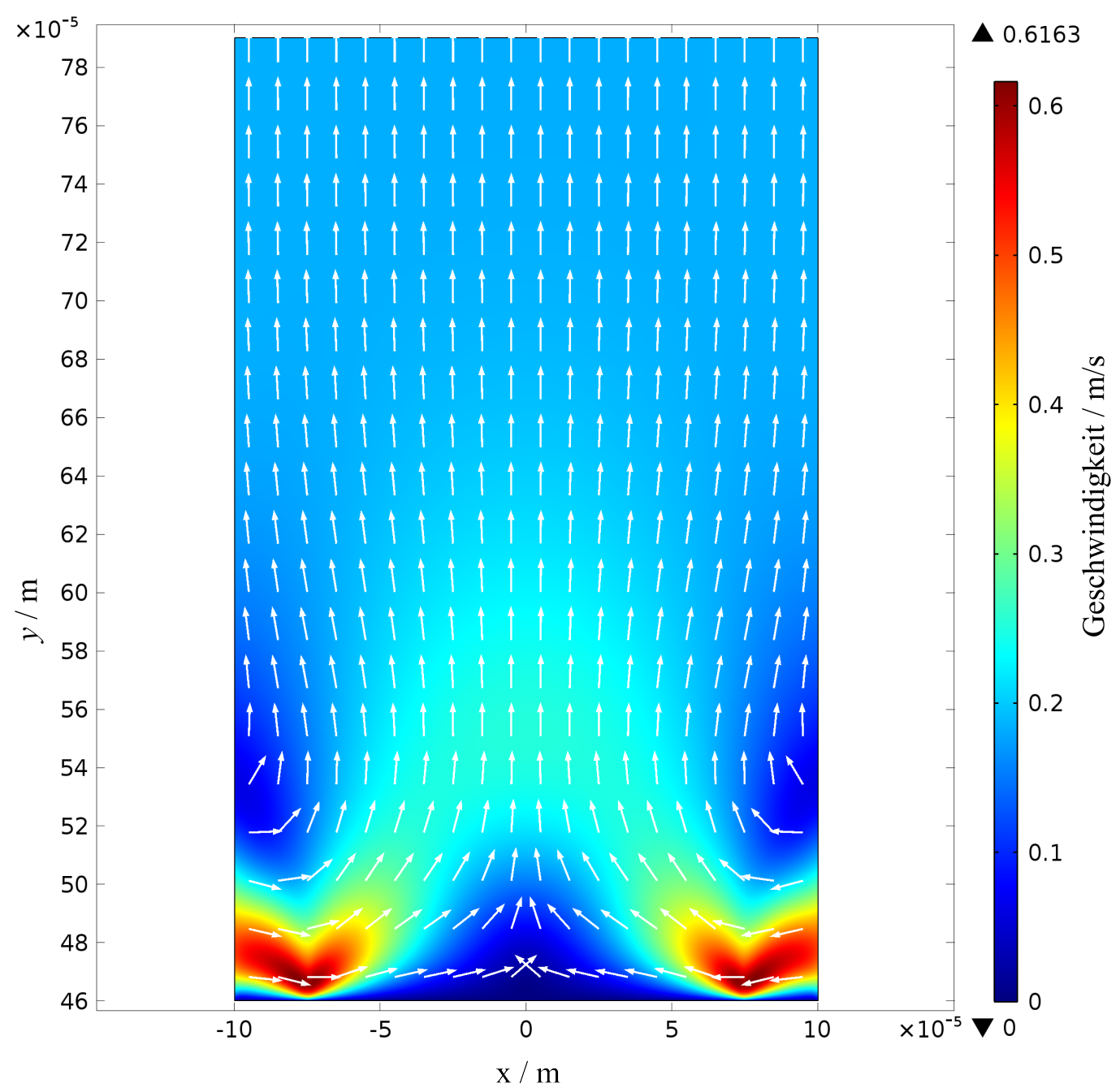

Abbildung 8.9.: Resultierendes Strömungsfeld der 2DSchallfeldsimulation eines $150 \mu \mathrm{m}$ breiten Schallwandlers bei $1.83 \mathrm{GHz}$ und $0.1 \mathrm{~nm}$ Auslenkung. Die Geschwindigkeit ist in der Einheit $\mathrm{ms}^{-1}$ angegeben.

mende Flüssigkeit von der Seite wird die Flüssigkeit in Richtung Küvettenmitte gepumpt. Der entstehende Jet nach oben ist etwas schmaler als die Gesamtbreite der aktiven Fläche. Die maximale Geschwindigkeit wird direkt an der Wandleroberfläche mit etwa $19 \mathrm{~cm} \mathrm{~s}^{-1}$ erreicht. In größerer Entfernung vom Wandler ist die Geschwindigkeit mit etwa $4 \mathrm{~cm} \mathrm{~s}^{-1}$ deutlich langsamer.

Die Strömung direkt über dem Wandler in Abbildung 8.11 zeigt einige Auffälligkeiten. So ist die Strömung auf den Wandler zu schneller als die Strömung vom Wandler weg. Der gleiche Effekt war auch bereits in den akustischen Simulationen in Kapitel 8.1.2 zu beobachten. Der Hauptgrund hierfür ist die Tatsache, dass die 


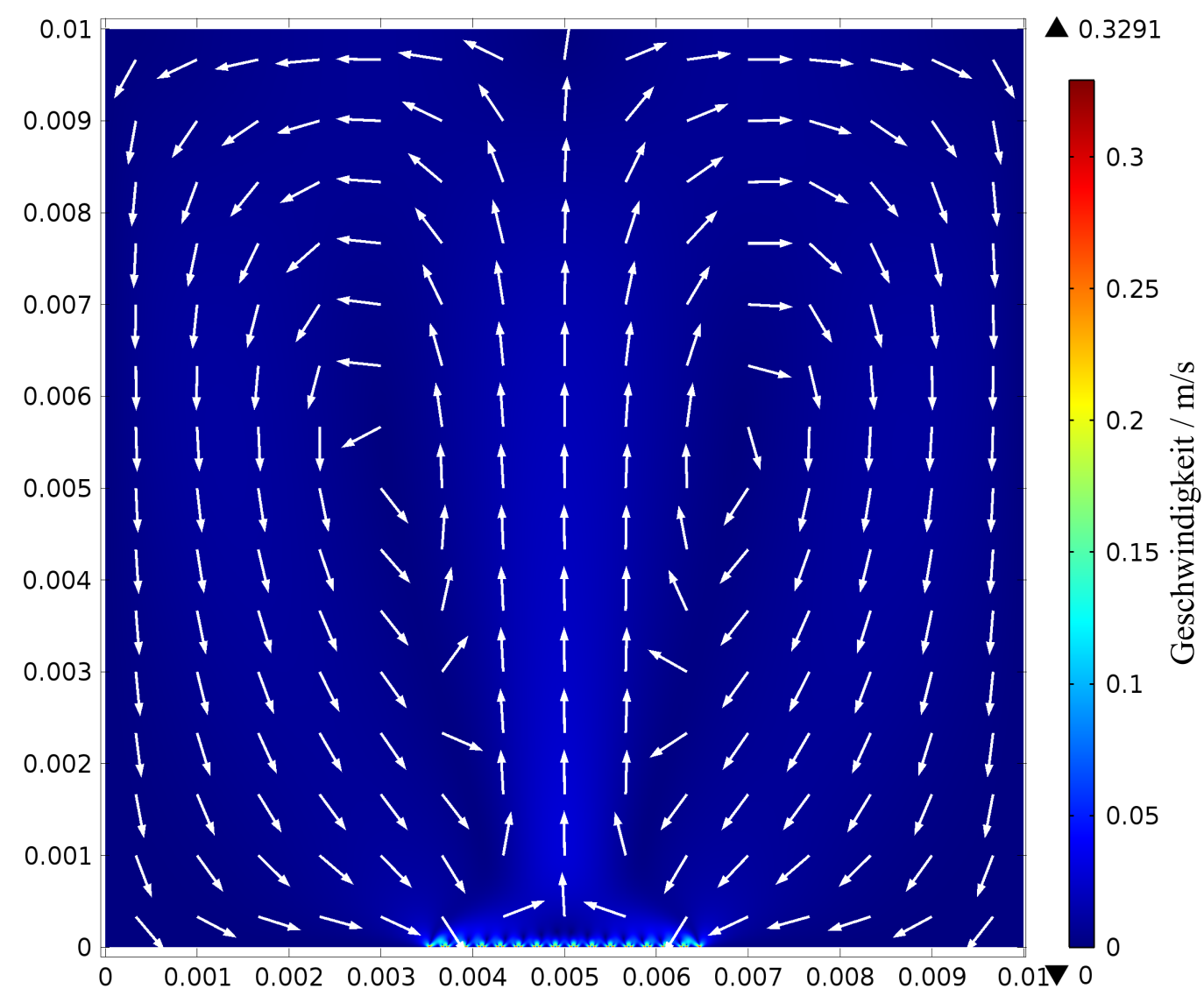

Abbildung 8.10.: Simulierte Strömung über dem Array-Schallwandler. Die Simulation wurde mit einer Volumenkraft von $1.7 \times 10^{8} \mathrm{~N} \mathrm{~m}^{-3}$ durchgeführt.

Breite eines Wandlers größer ist als der Abstand zwischen zwei Wandlern, was in einer 2D-Simulation bei konstantem Volumenstrom eine höhere Strömung auf den Wandler zu zur Folge hat. Des Weiteren sind anhand der eingezeichneten Strömungslinien einzelne Wirbelringe zwischen den einzelnen Wandlern zu erkennen, die in ihrer Form und Größe stark an die beobachteten Wirbelringe in Abbildung 7.6 erinnern. Außerdem lassen sich Strömungslinien finden, die in wellenförmigen Bahnen vom Rand des Wandlers in Richtung Wandlermitte und von dort nach oben führen. In Experimenten mit Partikeln sind Partikel zu beobachten, die ähnlichen Strömungslinien folgen. 


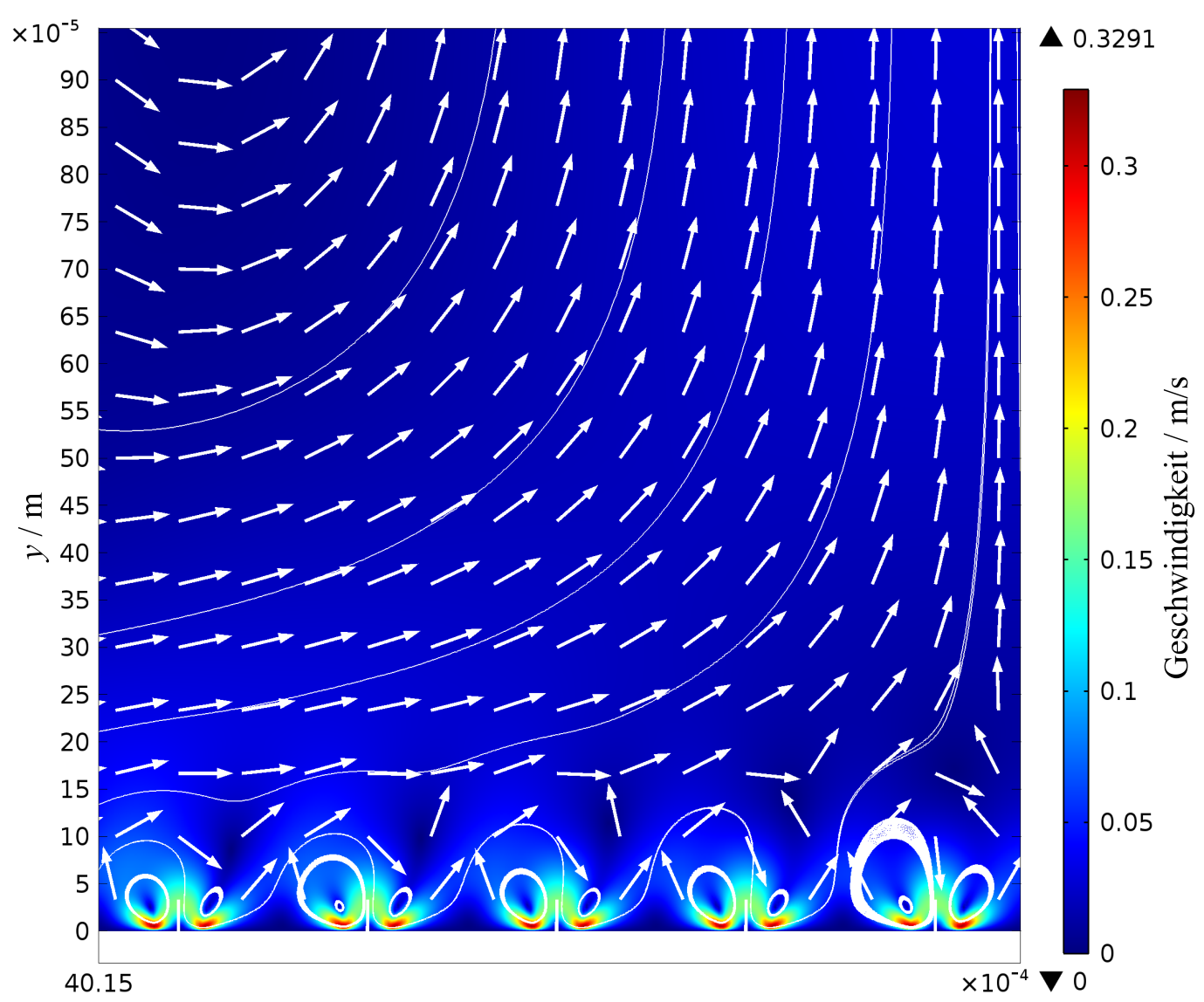

Abbildung 8.11.: Simulierte Strömung über dem Array-Schallwandler. Die Simulation wurde mit einer Volumenkraft von $1.7 \times 10^{8} \mathrm{~N} \mathrm{~m}^{-3}$ durchgeführt. Durch die eingezeichneten Strömungslinien sind direkt über dem Wandler Wirbelringe zu erkennen.

\subsubsection{Zwillings-Schallwandler}

Die Simulationen der Zwillings-Schallwandler wurden mit Laminar Flow und Transport of Concentrated Species durchgeführt. Das Gitter besteht insgesamt aus 162693 Elementen mit einer Maximalgröße von $6 \times 10^{-5} \mathrm{~m}$. Durch verschiedene Verteilungsvorgaben wurde direkt im Bereich der angelegten Volumenkraft in Richtung der Volumenkraft eine Elementgröße von $3 \times 10^{-7} \mathrm{~m}$ und in der Breite von etwa $4 \times 10^{-6} \mathrm{~m}$ erreicht. Die Rechenzeit betrug etwa 40 Stunden. Für die Simulation der Zwillings-Schallwandler wurden zwei einzelne Wandler mit einer Breite von je $100 \mu \mathrm{m}$ mit einer Lücke zwischen den Wandlern von $20 \mu \mathrm{m}$ simuliert. Diese Simulationen wurden an die beobachtete, mit Tinte sichtbar gemachte gepulste Strömung angepasst. Es wurde eine Pulsfrequenz von $10 \mathrm{~Hz}$ benutzt, die Pulsform wurde an die gemessenen Pulsformen des realen Verstärkers angepasst (siehe Abbildung 7.11(b)). 


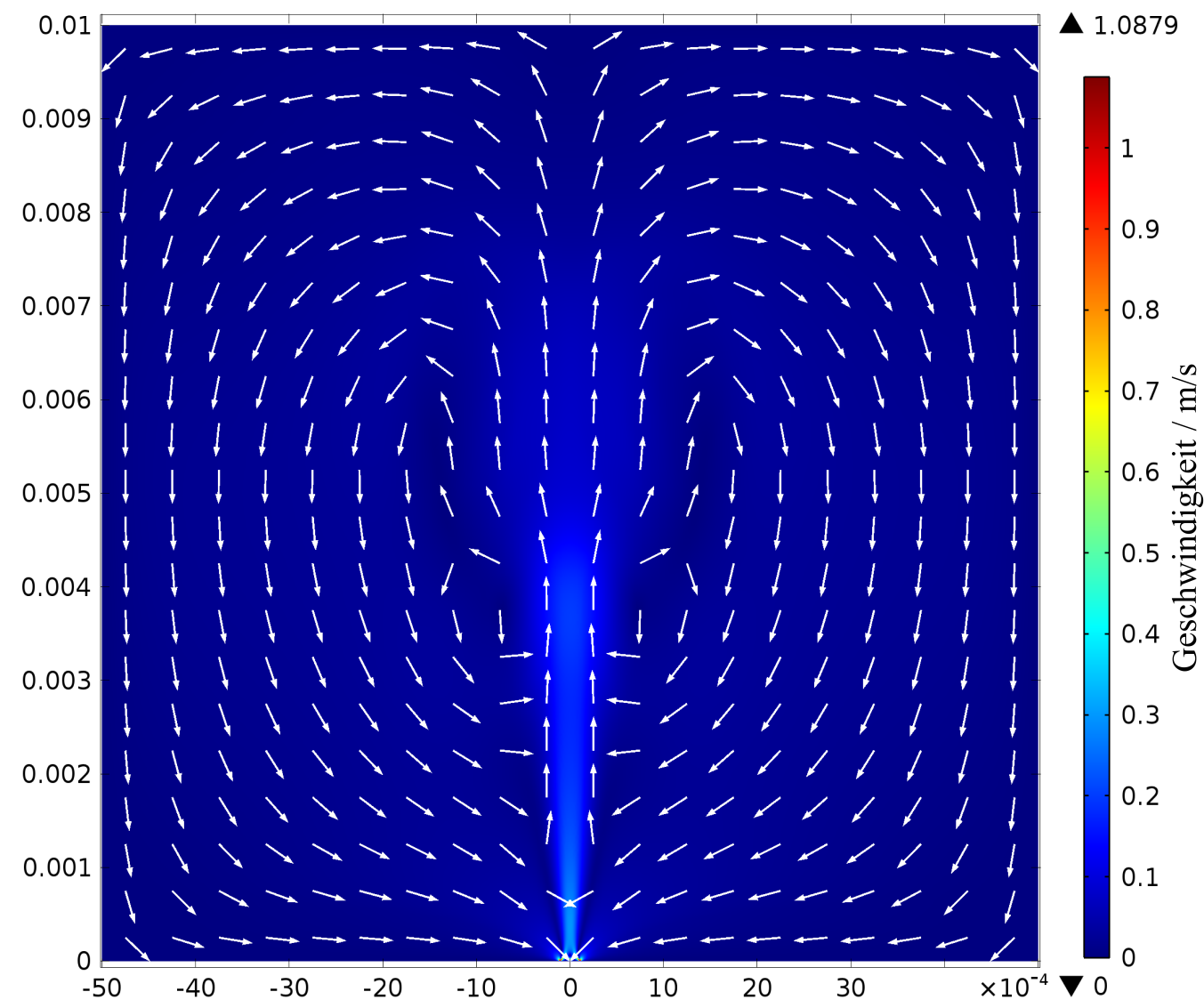

Abbildung 8.12.: Simulierte Strömung über dem ZwillingsSchallwandler nach $267 \mathrm{~ms}$. Die Simulation wurde mit einer Volumenkraft von $2.3 \mathrm{GN} \mathrm{m}^{-3}$ durchgeführt. Im unteren Bereich befand sich zu Beginn der Simulation ein Gebiet höherer Viskosität. Die Viskosität dieser Flüssigkeit betrug $5 \times 10^{-3} \mathrm{Pas}$.

Für die Tinte wurde eine Viskosität von $5 \times 10^{-3} \mathrm{Pas}$ angenommen. Bei einer Volumenkraft von $2.3 \mathrm{GN} \mathrm{m}^{-3}$ ergab sich eine ähnliche Form der Strömung und ein ähnlicher zeitlicher Verlauf. Aus diesem Grund wurde diese Volumenkraft für die Simulationen der Strömung benutzt. Die Volumenkraft entspricht bei der genutzten Frequenz einer Auslenkung des Wandlers von etwa 2.7 nm. Insgesamt gab es bei den Simulationen einen relativ kleinen Wertebereich der geeigneten Volumenkraft. Wurde die Kraft zu gering gewählt, wurde nur ein sehr kleiner Bereich direkt oberhalb des Wandlers zum Strömen angeregt. Wurde die Volumenkraft zu groß gewählt, treten Instabilitäten auf und die resultierende Strömung wird zur Seite abgelenkt. Beide Effekte waren auch in den Experimenten zu beobachten. Bei einer zu niedrigen Eingangsleistung oder falscher Frequenz kann sich keine richtige Strömung ausbilden und es sind nur kleine Wirbel an der Wandlerober- 
fläche zu beobachten. Leichte Instabilitäten konnten in Experimenten ebenfalls beobachtet werden. Allerdings ist bei den in den vorherigen Kapiteln gezeigten Messungen die Leistungsfähigkeit der Schallwandler bereits erreicht.

Abbildung 8.12 zeigt die Strömung in der gesamten Küvette. Es ist ein relativ scharf begrenzter Jet in der Mitte der Küvette zu erkennen, dessen Geschwindigkeit im unteren Drittel bis etwas über $30 \mathrm{cms}^{-1}$ erreicht. Nach oben wird der Jet etwas breiter und langsamer. Durch den gepulsten Betrieb ergeben sich mehrere aufsteigende Wirbel, die anhand der eingezeichneten Pfeile zu erahnen sind. Die Abbildungen 8.13(a) und 8.13(b) zeigen einen Vergleich zwischen

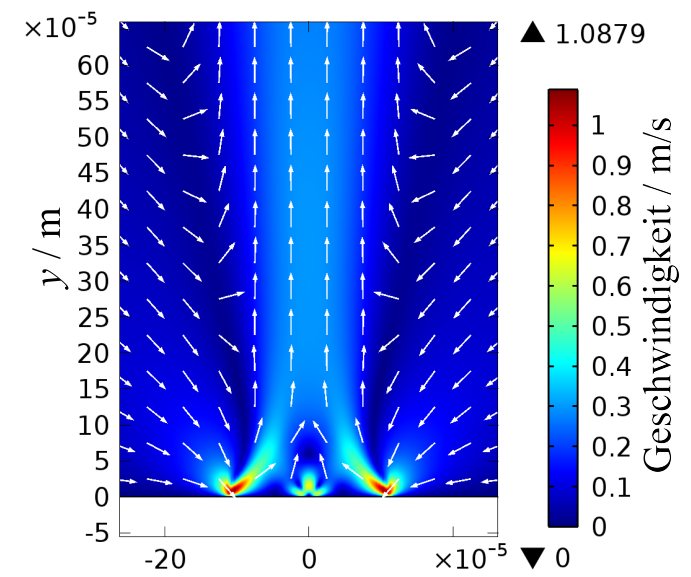

(a) Mit Comsol simulierte Strömung über einem Zwillings-Schallwandler bei $1.83 \mathrm{GHz}$ und $2.3 \mathrm{GN} \mathrm{m}^{-3}$ Volumenkraft. Die Lücke zwischen den Wandlern ist $20 \mu \mathrm{m}$ groß.

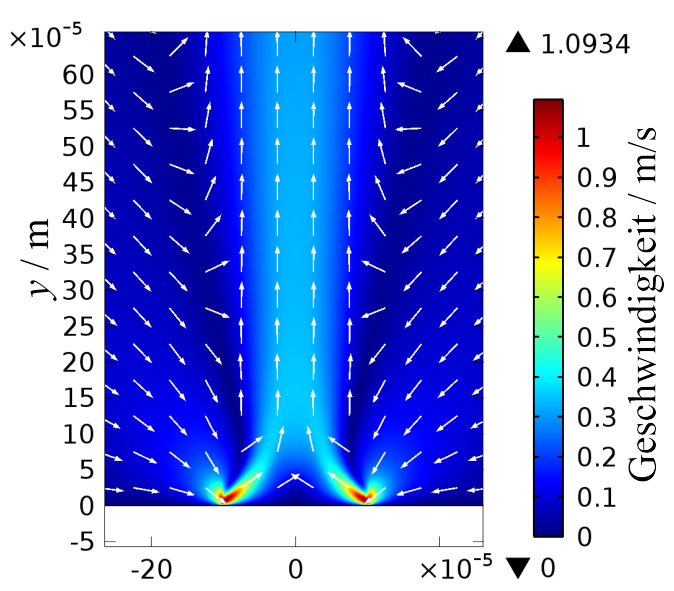

(b) Mit Comsol simulierte Strömung über einem Einzel-Schallwandler bei $1.83 \mathrm{GHz}$ und $2.3 \mathrm{GN} \mathrm{m}^{-3}$ Volumenkraft.

Abbildung 8.13.: Die Gesamtbreite der Wandler beträgt $200 \mu \mathrm{m}$.

einem Zwillings-Schallwandler, wie für die Simulation der Strömung in Abbildung 8.12 benutzt wurde, und einem Einzel-Schallwandler gleicher Gesamtbreite. In Teilabbildung 8.13(a) ist der nach oben gerichtete Jet zu erkennen. Die erreichte Geschwindigkeit innerhalb des Jets beträgt etwas über $30 \mathrm{~cm} \mathrm{~s}^{-1}$. Die Maximalströmung von $1.09 \mathrm{~m} \mathrm{~s}^{-1}$ wird wieder direkt an der Wandleroberfläche im Bereich der Zuströmung erreicht und wird in der Realität durch den Übergang zur 3D-Geometrie sicherlich kleiner sein. In der Mitte zwischen den Wandlern ist eine Rückströmung zu erkennen, die ebenfalls relativ schnell ist. Die Teilabbildung 8.13(b) zeigt, dass die Strömungsgeschwindigkeit über einem Einzelwandler gleicher Gesamtbreite etwas höher ist. In diesem Fall geht keine Energie in der Rückströmung verloren, hier werden im unteren Bereich des Jets in etwa $150 \mu \mathrm{m}$ Entfernung noch $40 \mathrm{~cm} \mathrm{~s}^{-1}$ erreicht. Durch eine Simulation mit zwei verschiedenen Flüssigkeiten unterschiedlicher Viskosität (ohne Oberflächenspannung und mit 


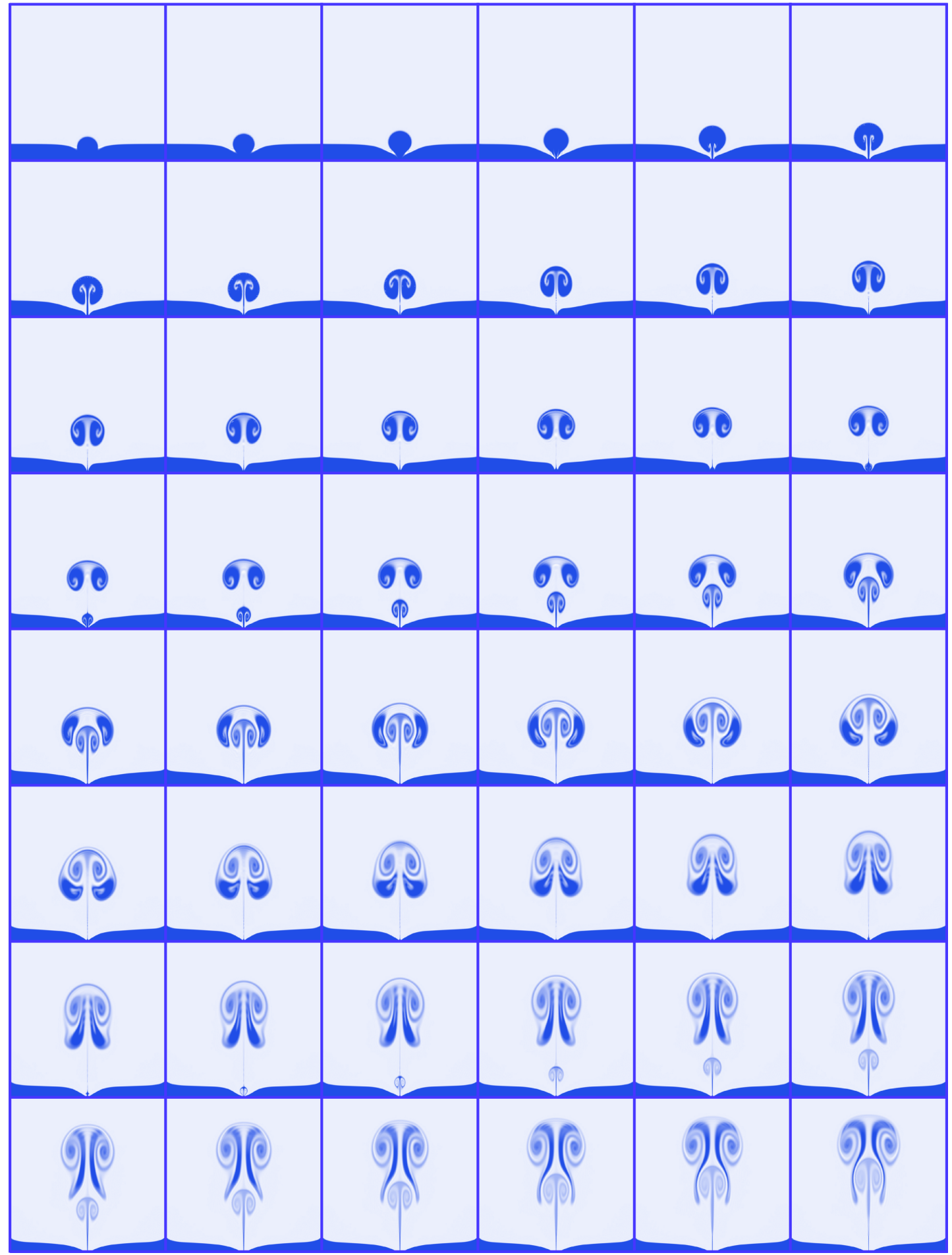

Abbildung 8.14.: Simulierte Strömung über dem ZwillingsSchallwandler, sichtbar gemacht mit Tinte. Die Simulation wurde mit einer Volumenkraft von $2.3 \times 10^{9} \mathrm{~N} \mathrm{~m}^{-3}$ durchgeführt. Die Viskosität der Tinte wurde mit $5 \times 10^{-3} \mathrm{Pas}$ abgeschätzt. Die Breite eines Einzelbildes beträgt $1 \mathrm{~cm}$. 
sehr langsamer Diffusionsgeschwindigkeit) lässt sich die gepulste Strömung ähnlich wie mit Einbringen von Tinte im Experiment sichtbar machen. Ein Ergebnis ist in Abbildung 8.15 gezeigt. Durch die höhere Viskosität der blau dargestellten Flüssigkeit ist der erste Puls relativ langsam und kommt nicht einmal bis zur Hälfte der Küvettenhöhe, bevor der zweite Puls den nächsten Wirbel nach oben beschleunigt. Dieser holt den ersten Puls auf etwa halber Küvettenhöhe ein und schießt durch diesen hindurch. Der erste Puls wird nun vom zweiten mitgezogen. Der dritte Puls schließt ebenfalls zu den anderen auf und drückt die durch den zweiten Puls entstandenen Schleppen etwas auseinander. In dieser Abbildung ist die innere Wirbelstruktur der einzelnen Pulse deutlich sichtbar. Die Auswertung

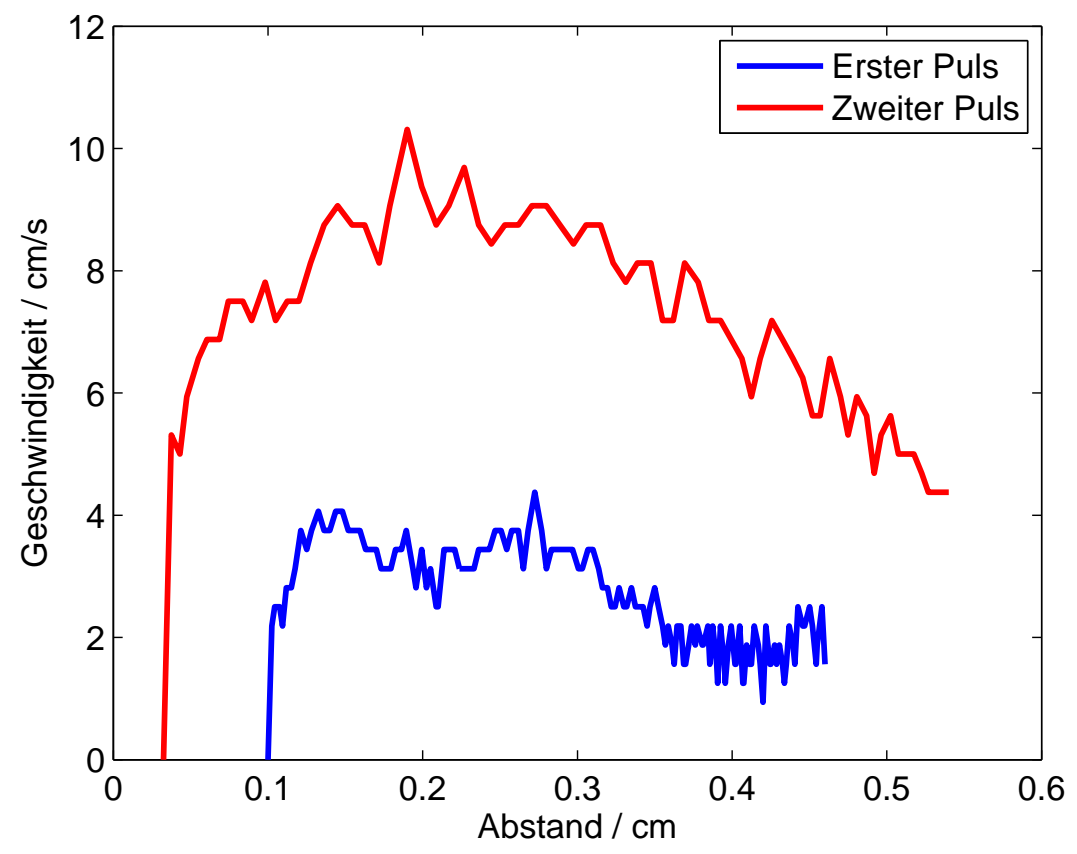

Abbildung 8.15.: Tintenfront-Verfolgung der Simulation mit Comsol.

der Geschwindigkeit der simulierten Tintenfront zeigt einen recht langsamen ersten Puls, der nur eine Maximalgeschwindigkeit von etwa $4 \mathrm{~cm} \mathrm{~s}^{-1}$ erreicht. Der zweite Puls ist mit fast $11 \mathrm{~cm} \mathrm{~s}^{-1}$ deutlich schneller. Diese Geschwindigkeit wird in etwa $2 \mathrm{~mm}$ Entfernung erreicht. Danach nimmt die Geschwindigkeit wieder ab. Die Ergebnisse der Simulationen zeigen, dass die approximativen VolumenkraftRechnungen das beobachtete Verhalten recht gut reproduzieren können und für den Fall des Lighthill-Streamings eine Alternative zu den aufwändigen akustischen Rechnungen darstellen. 



\section{Kapitel 9 \\ Diskussion der Ergebnisse im Gigahertzbereich}

\subsection{PIV-Aufnahmen}

PIV-Messungen der Strömungen an den Gigahertz-Schallwandlern in Kapitel 7.2.1 zeigen laminare Jet-Strömungen mit relativ hoher Geschwindigkeit. Trotz der hohen Schalldämpfung sind die erzeugten Strömungen auch in größerer Entfernung zu beobachten, wie in der Theorie (Kapitel 2.1.3) erläutert wurde [107]. Problematisch sind die sehr kleinskaligen Strömungen direkt an der Wandleroberfläche. Diese spielen sich innerhalb der ersten zehn Mikrometer ab, und eine leichte Verkippung des Wandlers führt dazu, dass die Vorder- oder Hinterkante des Wandlers diesen Bereich verdeckt. Dadurch kann die Strömung an der Oberfläche nur begrenzt beobachtet werden. Außerdem sind sehr kleine Partikel nötig um bei der hohen optischen Aufösung eine PIV-Messung durchführen zu können. Die benötigte optische Auflösung liegt dann im Bereich der optischen Auflösungsgrenze. Trotzdem ist es gelungen, die Wirbel an der Wandleroberfläche sichtbar zu machen, allerdings konnten keine Geschwindigkeiten bestimmt werden. Der Einfluss der einzelnen Wandler des Arrays und der durch diese erzeugten Wirbel auf die Strömung ist aber in der PIV-Aufnahme des unteren Bereichs der Strömung zu erkennen. Auch die großräumige Strömung in der gesamten Küvette konnte mit Hilfe dieser Messungen sichtbar gemacht und die Geschwindigkeiten bestimmt werden. Nur im unteren Bereich der Strömung kommt eine zu niedrige gemessene Geschwindigkeit durch die unter Umständen für diese Geschwindigkeiten zu niedrige Aufnahmerate zustande.

Die Geschwindigkeiten in einigem Abstand über dem Zwillings-Schallwandler wurden über Tintenfront-Tracking bestimmt. Selbst in knapp einem Millimeter Entfernung wurden bei diesen Wandlern noch hohe Geschwindigkeiten von bis $\mathrm{zu} 35 \mathrm{~cm} \mathrm{~s}^{-1}$ gemessen. 
Der Verlauf der gemessenen kinetischen Energiedichte über der Leistung zeigt bei niedrigen Leistungen einen klar ansteigenden Verlauf, der sich bei höheren Leistungen (im Pulsbetrieb) jedoch nicht eindeutig fortsetzt. Wird die mittlere Leistung über die Dauer eines Pulses und die mittlere kinetische Energiedichte betrachtet, liegen die Messwerte in der Nähe der Messwerte des unmodulierten Betriebs. Bei Betrachtung des jeweiligen Maximalwertes - der maximalen Leistung und der maximalen kinetischen Energiedichte - zeigt sich kein steigender Verlauf mit zunehmender Leistung. Für diesen Effekt gibt es zwei Möglichkeiten: Entweder die kinetische Energiedichte geht in Sättigung weil der Wandler die zugeführten Leistungsspitzen nicht umsetzten kann oder die verwendete Aufnahmerate ist zu gering, so dass die entstehenden Spitzengeschwindigkeiten nicht mehr aufgelöst werden können.

Das sporadische Auftreten von hohen Geschwindigkeiten in der PIV-Auswertung legt den offenbar falschen Schluss nahe, dass es sich bei diesen Werten um Messfehler handelt. Da die verwendeten Messungen qualitative Mängel aufwiesen, wurden die erhaltenen Maximalwerte spaltenweise aufgetragen. Diese Auftragung zeigt, dass es sich bei den hohen Messwerten höchstwahrscheinlich um die tatsächlich vorhandenen Geschwindigkeiten handelte. Die mit diesen Werten berechneten Reynolds-Zahlen zeigen zu Beginn, bei niedrigen Leistungen, den erwarteten linearen Verlauf. Bei hohen Leistungen steigen auch diese nicht weiter an, was ebenfalls für eine Begrenzung der Geschwindigkeit durch den Schallwandler oder die Aufnahmerate spricht.

Bei den Zwillings-Schallwandlern ist es nicht gelungen, mit Hilfe von PIVAufnahmen die Strömung in der gesamten Küvette aufzunehmen. Vor allem durch den gepulsten Betrieb der Zwillings-Schallwandler ist die Auswertung der PIVAufnahmen relativ schwierig und weist ein hohes Maß an Messrauschen auf. Die Geschwindigkeiten bei diesem Betrieb schwanken sehr stark, was sehr unterschiedliche Anforderungen an die Aufnahmegeschwindigkeit, die Messfenstergröße und die Verschiebung des Messfensters zur Folge hat. Außerdem sorgen die geringe Ausdehnung von nur etwa $200 \mu \mathrm{m}$ und die scharfe Begrenzung der Jet-Strömung in Kombination mit der Tiefenschärfe des Objektives und des sehr starken räumlichen Geschwindigkeitsgradienten für viele Fehler der PIV-Software, weshalb die tatsächliche Geschwindigkeit im unteren Bereich nur selten gemessen werden konnte. Aufgrund von zeitlichen Engpässen konnten leider keine Messungen der Gigahertz-Schallwandler mit Laserlichtschnitt mehr durchgeführt werden. Mit diesem sollten die bestehenden Probleme wie zu geringe Zeitauflösung, schlechter Kontrast und zu hohe Tiefenschärfe gelöst werden können. 


\subsection{Elektrochemische Messungen}

Die elektrochemischen Messungen erwiesen sich als gut reproduzierbar und geeignet, um Strömungen und Wandlerstrukturen zu charakterisieren [110]. So konnten die relativen Geschwindigkeiten in Abhängigkeit vom Abstand und der Position über dem Wandler gemessen werden. Auch potentielle Stehwelleneffekte bei sehr kleinem Abstand zum Wandler waren zu beobachten. Allerdings lässt der bei den elektrochemischen Messungen gemessene Strom im Gigahertzbereich nicht immer Rückschlüsse auf die Strömungsgeschwindigkeit zu. Voraussetzung für einen hohen gemessenen Strom ist eine hohe Zuströmung frischer Flüssigkeit, um neue Ionen an die Messelektrode zu liefern. Bei Strömungen in sehr schmalen Spalten können allerdings sehr schnelle kleine Wirbel zwischen den Grenzflächen und eventuell innerhalb des Diffusionsbereiches auftreten, die aber nur zwischen diesen Grenzflächen für eine hohe Strömungsgeschwindigkeit sorgen und somit keine frische Flüssigkeit zur Elektrode befördern. Nur bei einem relativ hohen Abstand vom Schallwandler, wenn von einer freien Strömung ausgegangen werden kann, lässt sich der gemessene Strom direkt auf die Geschwindigkeit zurückführen. Eventuell nimmt der Strom aus diesem Grund bei sehr kleinen Abständen zum Wandler hin ab, obwohl dort die höchsten Geschwindigkeiten auftreten sollten. Wird der Strom aber in einem konstanten Abstand gemessen, lässt sich dieser auf eine relative Strömungsgeschwindigkeit beziehen. Eine Kalibrierung, um den gemessenen Strom in eine Geschwindigkeit umzurechnen, wurde noch nicht durchgeführt.

Durch die relativ große Messelektrode tritt ein weiterer Effekt auf. So bildet die Messelektrode eine dem Wandler gegenüber liegende Grenzfläche und beeinflusst die Strömung stark. Je nach Position der Elektrode wird die Strömung zu verschiedenen Seiten abgelenkt und es bilden sich unterschiedliche Wirbel aus. Trotzdem konnten die Array-Struktur der verwendeten Array-Schallwandler und deren Einzelströmungen gut verifiziert werden. Die Messung der Zwillings-Schallwandler zeigt in einer Richtung drei Maxima, die sich eventuell durch entstehende Wirbel zwischen Elektrode und Wandler erklären lassen. Auch die Struktur des Wandlers mit zwei in Reihe geschalteten elliptischen abstrahlenden Zonen könnte für diese drei Maxima verantwortlich sein. So ist in den Messungen mit Tinte über dem Wandler ebenfalls eine Struktur von drei dunkleren Bereichen innerhalb des Jets zu erkennen, was ebenfalls auf drei Bereiche maximaler Geschwindigkeit hinweisen könnte. 


\subsection{Vergleich zwischen Simulation und Experi- ment}

Die experimentell beobachteten Strömungen konnten in den Simulationen recht gut reproduziert werden. Mit Hilfe von Simulationen der kompletten Wandlerstruktur inklusive Piezoelektrik und Trägermaterial bei einer Frequenz von $400 \mathrm{MHz}$ konnte die Gültigkeit der Vereinfachung einer von der Wandleroberfläche abgestrahlten ebene Welle verifiziert werden. Obwohl das Schallfeld innerhalb des Wafermaterials an Komplexität zunimmt, bleibt die stark schwingende Oberfläche räumlich begrenzt und erzeugt eine größtenteils auf den Bereich direkt über dem Wandler beschränkte Strömung. Der Vergleich zwischen gekoppelten akustischen und Strömungs-Simulationen und reinen Strömungs-Simulationen mit exponentiell abklingender Volumenkraft als genähertem Antrieb durch die Schalldämpfung vor den Gigahertz-Schallwandlern zeigt trotz der eigentlich recht komplexen räumlichen Struktur der Volumenkraft die Eignung auch dieser Vereinfachung. Bei geeigneter Wahl der Auslenkung und Volumenkraft stimmen die groben Strukturen und Geschwindigkeiten der resultierenden Strömung überein. Leider war es aufgrund fehlender Verfügbarkeit von geeigneten Laservibrometern nicht möglich, die tatsächliche Auslenkung der Wandler zu bestimmen. Aufgrund der eingekoppelten elektrischen Leistung und der aktiven Fläche der verwendeten Wandler kann unter der Annahme, dass die elektrische vollständig in akustische Energie umgewandelt wird, die erreichte Auslenkung berechnet werden. Diese Rechnung ergibt Auslenkungen von etwa einem Nanometer. Vergleiche mit den Simulationen bestätigen die Größenordnung dieser Rechnung. Zum Experiment ähnliche Strömungsgeschwindigkeiten ergaben sich in den Simulationen zwischen 0.1 und 1 Nanometer Auslenkung. Bei der Simulation des Array-Schallwandlers konnten Wirbelstrukturen direkt an der Wandleroberfläche, die im Experiment nur im Ansatz zu beobachten waren, genauer sichtbar gemacht werden. Die dabei berechneten Geschwindigkeiten dürften allerdings speziell innerhalb der Zuströmung zu den einzelnen Wandlern durch die Dimensionsreduktion von 3d auf 2d $\mathrm{zu}$ hoch sein. Die resultierende Gesamtströmung und die Beiträge der einzelnen Wandler zu dieser sind anhand der Simulation gut nachzuvollziehen. Für die Simulation der gepulsten Strömungen am Zwillings-Schallwandler wurde die Pulsform des am Wandler anliegenden elektrischen Signals in etwa nachgebildet und als zeitliche Komponente der auf die Flüssigkeit wirkenden Volumenkraft hinzugefügt. So konnte auch in der Simulation der gepulste Betrieb nachgebildet werden. Um die Struktur der Strömungen an dem Zwillings-Schallwandler auch in der Simulation sichtbar zu machen, wurde eine Simulation mit zwei verschie- 
denen Flüssigkeiten unterschiedlicher Viskosität durchgeführt. Da die Viskosität der zweiten Flüssigkeit (Tinte) unbekannt war und geschätzt wurde, lassen sich die beobachteten Effekte im Wesentlichen qualitativ vergleichen. So konnte in der Simulation der Effekt, dass der zweite Puls durch den ersten hindurch geschossen wird, nachgebildet werden. Die Vermutung, dass es sich hierbei um einen Viskositätseffekt handelt wurde so bestätigt. Die Ähnlichkeit der Strömungsfelder in Simulation und Experiment ist klar zu erkennen. Allerdings breitet sich der Puls in der Simulation sehr viel stärker in der Breite aus und bleibt im oberen Bereich der Küvette schneller, als es im Experiment zu sehen ist.

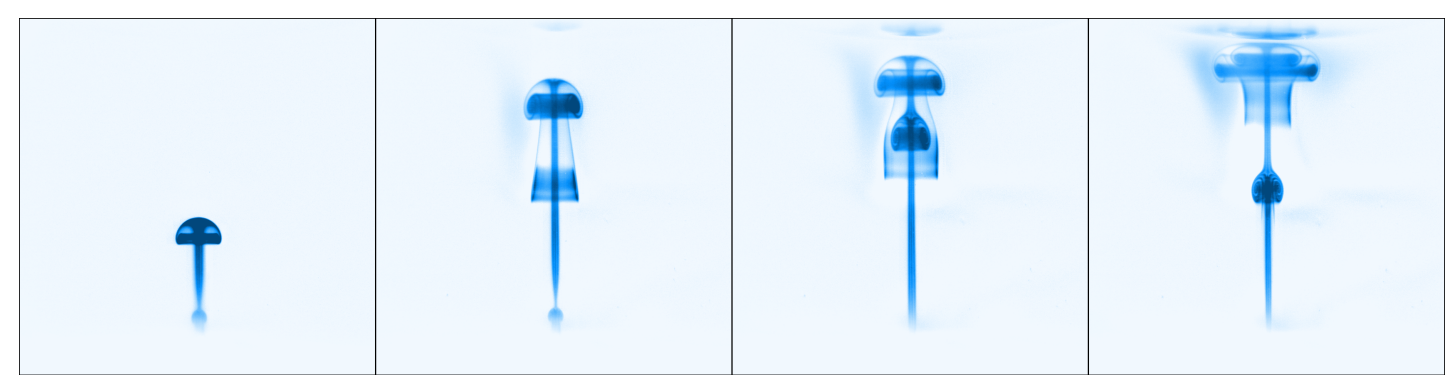

(a) Ausgewählte Bilder aus der Bildreihe in Abbildung 7.12. Zeitschritte: $0 \mathrm{~ms}, 105 \mathrm{~ms}, 155 \mathrm{~ms}$ und $235 \mathrm{~ms}$.

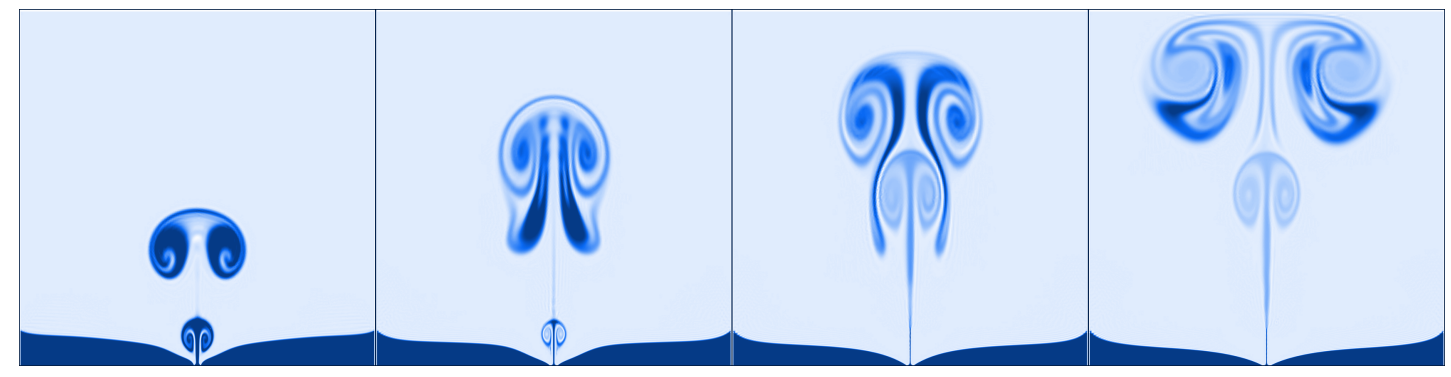

(b) Ausgewählte Bilder aus der Bildreihe in Abbildung 8.14. Zeitschritte: 0 ms, 95 ms, 140 ms und $235 \mathrm{~ms}$.

Abbildung 9.1.: Vergleich der Strömungsstrukturen eines ZwillingsSchallwandlers in Experiment und Simulation. Hierbei wurden immer möglichst ähnliche Bilder ausgewählt (siehe auch [108]). 



\section{Kapitel 10 Zusammenfassung und Ausblick}

Im ersten Teil dieser Arbeit wurden die akustischen Strömungen an einer Sonotrode bei gleicher Auslenkungsamplitude mit und ohne Kavitation verglichen. Dies wurde durch einen speziellen Versuchsaufbau ermöglicht, der über einen geschlossenen Wasserkreislauf verfügt und so Temperatur, Gasgehalt und Druck einstellbar machte.

Insbesondere konnte durch eine Erhöhung des statischen Drucks auf zwei Bar die Kavitation unterdrückt und zusätzlich durch Änderung von Gasgehalt oder Temperatur die Kavitationsschwelle verschoben werden. Mit Hilfe eines speziellen Fluoreszenzmikroskopaufbaus war es möglich, die Strömungen und die Kavitationsblasendynamik simultan und zeitaufgelöst aufzunehmen. Die Ergebnisse zeigen einen deutlichen Unterschied zwischen der reinen akustischen Strömung und der von sekundären Effekten überlagerten Strömung im Kavitationsblasenfeld. Die ab der Kavitationsschwelle entstehenden, stark schwingenden Blasen sorgen für eine massive Erhöhung des Dämpfungsfaktors um einen stark streuenden Faktor von im Mittel etwa 30. Dies entspricht bei $20^{\circ} \mathrm{C}$ einer Zunahme des Dämpfungsfaktors $\alpha$ von $7.6 \times 10^{-6} \mathrm{~m}^{-1}$ auf $2.3 \times 10^{-4} \mathrm{~m}^{-1}$. Der Schall wird stärker absorbiert und in kinetische Energie einer Gleichströmung umgesetzt. Durch Oszillation und Translation erzeugen diese Blasen zusätzliche Mikroströmungen hoher Geschwindigkeit, die einige Größenordnungen über der Geschwindigkeit der akustischen Strömungen liegen können. Die Kombination aus akustischen Strömungen, Bjerknes-Kräften, Impulserhaltung und Strahlungsdruck sorgt für komplexe, auf die Blasen wirkende Kräfte, die die Blasenbewegungen, die Strukturbildung innerhalb der Blasenwolke und somit durch Schalldämpfung und Schallstreuung wiederum das Schallfeld beeinflussen können. Dieses hat seinerseits wieder einen Einfluss auf die Entstehung von Blasen und damit auf die durch diese hervorgerufenen Strömungen, die wiederum für einen Transport der Blasen verantwortlich sind. Durch diese Wechselwirkung können räumliche und zeitliche Oszillationen des Blasenfeldes auftreten. Diese können sowohl mit dem anregenden Schallfeld in Zusammenhang stehen als auch turbulente 
Fluktuationen zeigen. Dies konnte mit Hilfe von Korrelationsanalysen und Fouriertransformationen nachgewiesen werden. So zeigen die Korrelationsanalysen bei Strömungen in Kavitationsblasenfeldern einen ständigen Wechsel zwischen korrelierter und unkorrelierter Strömung. Auch die Fouriertransformierten weisen neben Artefakten (zum Beispiel der Differenzfrequenz zwischen anregender Frequenz und Aufnahmerate) weitere Amplitudenmaxima bei anderen Frequenzen auf, was ein Hinweis auf zum Schallfeld harmonische Fluktuationen der Blasenschwingungen, beziehungsweise der Blasendichte, und der zum Teil daraus resultierenden Strömungsgeschwindigkeit ist.

Des Weiteren konnte ein Zusammenhang zwischen dem Blasenvolumen und der gespeicherten kinetischen Energie festgestellt werden. Auch eine starke räumliche Korrelation zwischen Blasenwolke und Geschwindigkeitsfeld wurde nachgewiesen. Ob das sich ausbreitende Strömungsfeld die Blasen transportiert, ob die Blasen das sich ausbreitende Strömungsfeld hervorrufen oder ob beide Effekte sich gegenseitig beeinflussen, konnte im Rahmen dieser Arbeit nicht geklärt werden. Für die Untersuchung des genauen Zusammenhangs von Einzelereignissen der Blasendynamik und den verursachten Strömungen sind noch genauere Kalibrierungen und eventuell höhere Aufnahmegeschwindigkeiten notwendig. Die Simulation des Schallfeldes ermöglichte die genauere Untersuchung einzelner Beobachtungen. So konnte beispielsweise die reproduzierbare Asymmetrie der Strömungen im kavitationsfreien Fall durch die Simulation geklärt werden.

Im zweiten Teil der Arbeit wurden reine akustische Strömungen ohne Kavitation bei Frequenzen im Gigahertzbereich experimentell und mit Hilfe von Simulationen untersucht. Die Funktionalität der speziell entwickelten Schallwandler konnte so bestätigt werden. Wie aufgrund des Zusammenhangs zwischen Frequenz und Dämpfungsfaktor zu erwarten war, ergeben sich trotz geringer Leistungen sehr hohe Strömungsgeschwindigkeiten. Die gesamte Energie wird nach kleiner Eindringtiefe absorbiert und der komplette Impuls des Schallfelds geht auf die Flüssigkeitsströmung über. Dies geschieht auf so kurzer Laufstrecke (etwa $50 \mu \mathrm{m}$ ), dass die Kräfte auf die Flüssigkeit gewissermaßen in einer dünnen Scheibe übertragen werden. So wird der Extremfall des „Lighthill“-Streamings realisiert, der stark einem Unterwasser-Flüssigkeitsstrahl (,submerged Jet“) ähnelt. Die Strömungsfelder wurden mit verschiedenen Methoden untersucht und die Strukturen der Strömung, die in der Tat einem Unterwasserstrahl entsprechen, abgebildet. Mit Hilfe elektrochemischer Messungen wurden Mikroströmungen in Wandlernähe untersucht, um die Anordnung der einzelnen Wandlerzellen zu charakterisieren und ihre Funktionalität zu verifizieren. Mit Hilfe von TintenfrontVerfolgung und PIV-Messungen konnten die Strömungsgeschwindigkeiten und 
Reynolds-Zahlen (bis zu 410 bei der maximal verwendeten Leistung) der Strömung bestimmt werden. Es zeigte sich mit zunehmender Leistung ein linearer Anstieg der Strömungsgeschwindigkeit, der bei hohen Leistungen in Sättigung geht. Die mittels PIV-Messungen und Tintenfront-Verfolgung bestimmten Geschwindigkeiten stimmen in etwa überein und betrugen bis zu 37 beziehungsweise $28 \mathrm{~cm} \mathrm{~s}^{-1}$. Die Struktur der entstehenden Wirbel innerhalb der Strömung konnte mit Tinte sichtbar gemacht und mit PIV-Messungen quantifiziert werden. Des Weiteren wurden die Strömungen dieser Wandler bei gegenüberliegenden Grenzflächen und innerhalb von schmalen Spalten qualitativ experimentell untersucht und simuliert. Die Ergebnisse - nicht Gegenstand dieser Arbeit - zeigen, dass sich im Wesentlichen vom Abstand der Grenzflächen abhängige Wirbel ausbilden. Um die Geschwindigkeiten dieser Wirbel und die Geschwindigkeiten nahe der Grenzflächen quantitativ zu untersuchen sind weitere Messungen mit kleineren Tracer-Partikeln und höherer optischer Aufösung nötig. Auch die Wirbel an der Wandleroberfläche bei freier Strömung müssen noch näher untersucht und besser abgebildet werden. Beides sollte mit dem jetzt vorhandenen Aufbau mit Lichtschnitt und dem Nd-YAG-Laser möglich sein. Die Strömungsgeschwindigkeiten innerhalb der Grenzschichten können nach erfolgreicher Kalibration mit Hilfe der elektrochemischen Messungen bestimmt werden. Der vorhandene Versuchsaufbau bietet die Chance, weitere, vereinfachte Schallwandler systematisch $\mathrm{zu}$ untersuchen und deren Geometrie und Frequenz zu optimieren. 



\section{Anhang A Konstruktion der Sonotroden}

Die Sonotroden wurden als Sandwichtransducer mit Rückmasse und Exponentialhornspitze konstruiert. Wegen der einfacheren Herstellung wurde als Material Aluminium verwendet und nur die Spitze wurde nachträglich durch einen schraubbaren Stahlzylinder ersetzt. Die Massekontaktierung erfolgte mit dünnen Kupferlötfahnen, die jeweils zwischen Piezokeramikring (Piezo) und Aluminiumbauteil geklemmt wurden. Zwischen den Piezokeramikringen wurde der Hochspannungskontakt, der gleichzeitig als Halterung genutzt wurde, positioniert. Zum Verspannen wurden einfache Stahlschrauben, die mit einer Isolierhülse versehen waren, verwendet. Das folgende Kapitel ist aus meiner Diplomarbeit übernommen worden [20].

\section{A.1. Anpassen des Exponentialhorns}

Ein Exponentialhorn ist durch die exponentielle Abnahme des Querschnittes mit der Länge charakterisiert. Der Querschnitt $S=\pi r^{2}$ der Exponentialhornspitze ergibt sich aus dem einfachen Exponentialgesetz [111] :

$$
S(x)=S_{1} \mathrm{e}^{-2 \epsilon x} .
$$

$\epsilon$ ist der Abklingkoeffizient und nach Gleichung (A.6) eindeutig bestimmbar. Anfangs- und Enddurchmesser $S_{1}$ und $S_{2}$ sind durch die verwendeten Piezokeramikringe und den gewünschten Spitzendurchmesser gegeben. Die Resonanzlänge $l$ (vergleiche Abbildung A.1) ergibt sich aus der Lösung der Gleichung für die nicht konstante Schallschnelle [100] mit der Dehnwellenlänge $\lambda_{\mathrm{D}}$ des Sonotrodenmaterials:

$$
l=\frac{\lambda_{\mathrm{D}}}{2} \sqrt{1+\left(\frac{1}{2 \pi} \ln \frac{S_{1}}{S_{2}}\right)^{2}} .
$$

Diese Formel geht allerdings von der Resonanzlänge $\lambda / 2$ aus (Schwingungsbäuche bei $S_{1}$ und $S_{2}$, Schwingungsknoten bei $x_{0}$ ), weshalb als Anfangspunkt des 


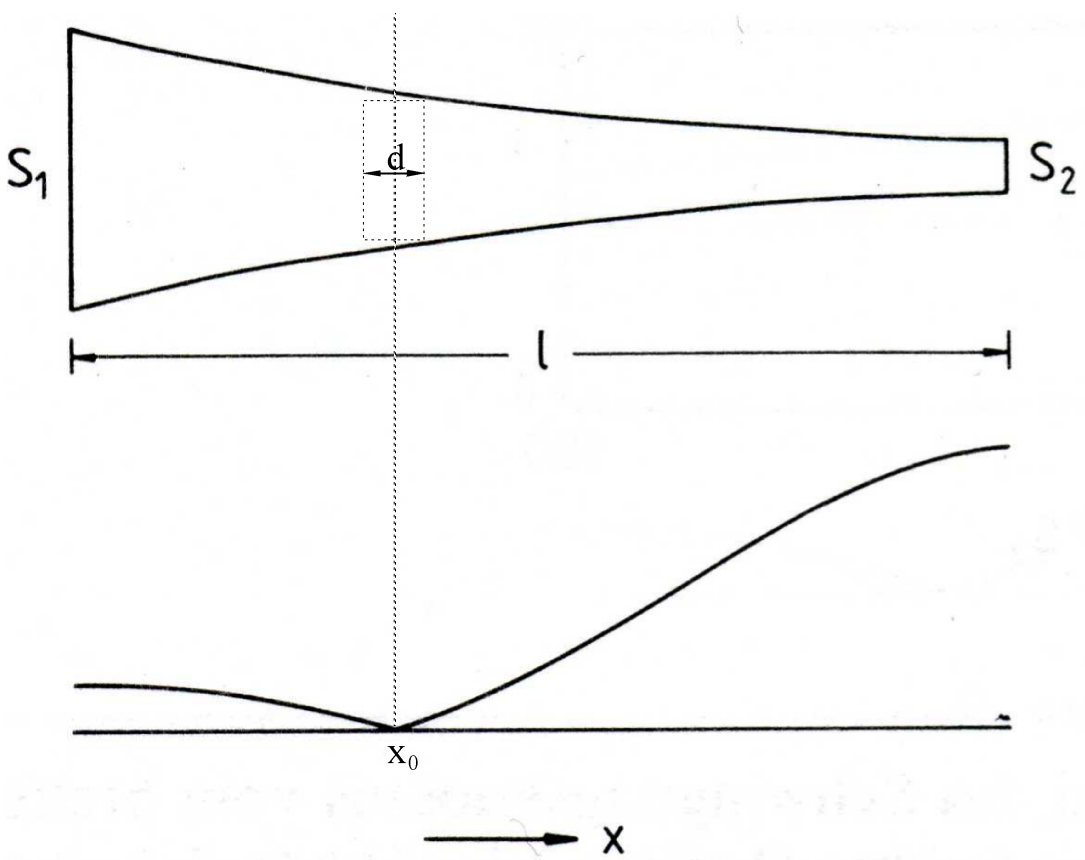

Abbildung A.1.: Form des Exponentialhorns und Verteilung der Schwingungsschnelle (schematisch, aus [100]). Die asymmetrische Lage des Schwingungsknotens bei $x_{0}$ ist gut zu erkennen.

Exponentialhorns idealisiert die Schwingungsknotenebene [100]

$$
x_{0}=\frac{l}{\pi} \arctan \left[\frac{2 \pi}{\ln \left(\frac{S_{1}}{S_{2}}\right)}\right]+\frac{d}{2}
$$

gewählt wurde. Damit wird der Schwingungsknoten zum einfachen Befestigen in die Nähe der Halterung zwischen den beiden Piezos verschoben. Daraus ergibt sich für die Länge der verwendeten Exponentialhornspitze:

$$
l_{\mathrm{H}}=l-x_{0}+\frac{d}{2} .
$$

Es wurde also $S_{1}$ so angepasst, dass $S\left(x_{0}\right)$ der Fläche der verwendeten Piezos entspricht. Außerdem wurde noch ein kurzes, zylinderförmiges Stück mit der Länge $d$ vorgesehen, um beim Vorspannen mit der Schraube die Sonotroden mit einem Schraubstock festklemmen zu können. Der Einfachheit halber wurde dieses allerdings zur Hälfte von der benötigten Gesamtlänge des Exponentialhorns abgezogen, so dass der Schwingungsknoten rein rechnerisch nun $l_{\mathrm{H}}-d / 2$ Abstand von der Sonotrodenspitze hat. Es ergibt sich also für die Exponentialhorngleichung:

$$
S(x)= \begin{cases}S\left(x_{0}\right) & \text { falls } 0 \leq x \leq d \\ S\left(x_{0}\right) \mathrm{e}^{-2 \epsilon(x-d)} & \text { falls } d<x \leq l_{\mathrm{H}}\end{cases}
$$




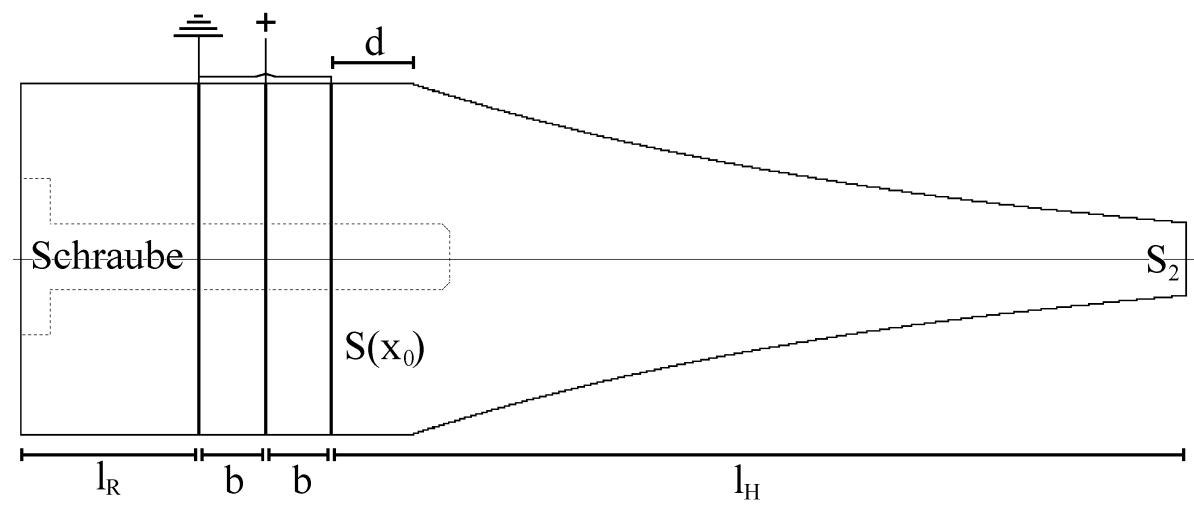

Abbildung A.2.: Die Bauteile des Exponentialhorns. $l_{\mathrm{R}}$ ist die Dicke der Rückmasse, $b$ die Dicke der Piezokeramikringe, $l_{\mathrm{H}}$ die Länge der Exponentialhornspitze, $d$ der zylindrische Teil zum Festklemmen bei der Montage und $S\left(x_{0}\right)$ und $S_{2}$ die Querschnittsfläche am Anfang, bzw. am Ende des Horns.

mit

$$
\epsilon=\frac{\ln \left(\frac{S_{1}}{S_{2}}\right)}{2 l} .
$$

\section{A.2. Berechnung der Rückmasse}

Die Auslenkung des freien Endes der Rückmasse lässt sich mit Hilfe der Formel

$$
\Xi_{\mathrm{R}}\left(-l_{\mathrm{R}}^{*}\right)=\frac{e V_{0}}{\omega b Z_{\mathrm{P}} K_{\mathrm{b}}\left(\frac{R Z}{K_{\mathrm{b}}} \sin \left(k_{\mathrm{R}} l_{\mathrm{R}}\right)+\cos \left(k_{\mathrm{R}} l_{\mathrm{R}}\right)\right)}
$$

berechnen [112], wobei $\left(-l_{\mathrm{R}}^{*}\right)=l_{\mathrm{R}}+b$, also die Entfernung vom Schwingungsknoten zwischen den Piezos ist. In Gleichung (A.7) ist $e$ der piezoelektrische Koeffizient, $V_{0}$ die Amplitude des elektrischen Potentials,

$$
K_{\mathrm{b}}=\frac{e^{2}}{\omega b \epsilon^{S} Z_{\mathrm{P}}}-\frac{\cos \left(k_{\mathrm{P}} b\right)}{\sin \left(k_{\mathrm{P}} b\right)}
$$

und

$$
R Z=\frac{Z_{\mathrm{R}} S_{\mathrm{R}}}{Z_{\mathrm{P}} S_{\mathrm{P}}}
$$

Außerdem ist $k_{m}=\frac{2 \pi}{\lambda_{m}}$ die gegenüber dem Artikel [112] vereinfachte Wellenzahl, $S_{m}$ die Fläche und $Z_{m}=\rho_{m} c_{m}$ die akustische Impedanz mit $m$ dem Index für den jeweiligen Abschnitt der Sonotrode (R: Rückmasse, H: Horn, P: Piezo).

Da die Auslenkung $\Xi_{\mathrm{R}}$, um geringe mechanische Verluste zu erhalten, möglichst 
klein sein muss, ergibt sich die Länge $l_{\mathrm{R}}$ durch Minimieren der Formel (A.7). Die meisten dieser Parameter sind Konstanten; deshalb lässt sich dieses Problem auf die Bestimmung der Extrema von

$$
\Psi_{\mathrm{R}}\left(l_{\mathrm{R}}\right)=\frac{R Z}{K_{\mathrm{b}}} \sin \left(k_{\mathrm{R}} l_{\mathrm{R}}\right)+\cos \left(k_{\mathrm{R}} l_{\mathrm{R}}\right)
$$

reduzieren. Geeignete Abmessungen der Rückmasse $l_{\mathrm{R}}$ ergeben sich dann aufgrund der Periodizität in jedem Extremum der in Abbildung A.3 dargestellten Funktion $\Psi_{\mathrm{R}}\left(l_{\mathrm{R}}\right)$. Da das erste Extremum $(\mathrm{A})$ bei negativer Länge liegt und das zweite (B) einer zu großen Länge der Rückmasse entspräche, wurde die aus mechanischen und konstruktiven Gründen minimal benötigte Größe mit möglichst geringer Abweichung vom ersten Maximum $(A)$ gewählt.

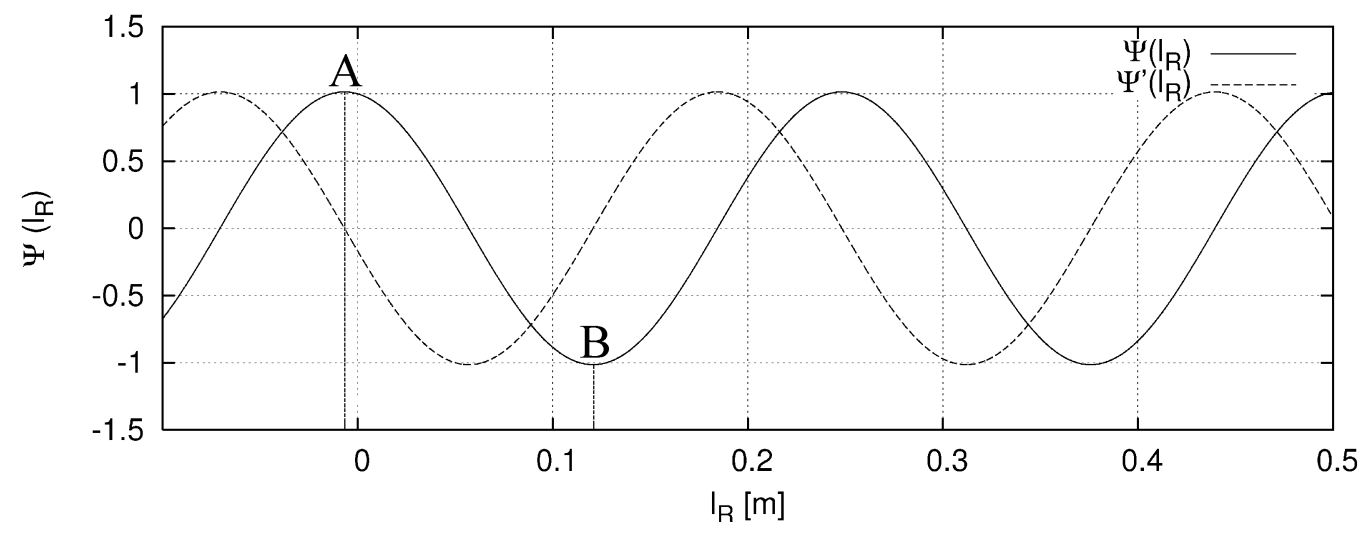

Abbildung A.3.: Zur Berechnung der Rückmasse: Funktion $\Psi_{\mathrm{R}}\left(l_{\mathrm{R}}\right)$ und ihre Ableitung $\Psi_{\mathrm{R}}^{\prime}\left(l_{\mathrm{R}}\right)$ (Beispiel für $20 \mathrm{kHz}$ Aluminium-Sonotrode).

\section{Verwendete Daten}

$$
\begin{aligned}
c_{\mathrm{Al}} & =5100 \mathrm{~m} \mathrm{~s}^{-1} \\
c_{\text {Piezo }} & =3900 \mathrm{~m} \mathrm{~s}^{-1} \\
\rho_{\mathrm{Al}} & =2710 \mathrm{~kg} \mathrm{~m}^{-3} \\
\rho_{\text {Piezo }} & =7800 \mathrm{~kg} \mathrm{~m}^{-3} \\
e & =17.65 \mathrm{C} \mathrm{m}^{-2} \\
\epsilon^{\mathrm{S}} & =61.85 \times 10^{-10} \mathrm{C}^{2} \mathrm{~N}^{-1} \mathrm{~m}^{-2}
\end{aligned}
$$




\section{Symbolverzeichnis}

A Schallschnelleamplitude am Schallwandler

$A_{0} \quad$ Schwingungsamplitude der Blasenwand

$\vec{F}^{\prime} \quad$ Kraft auf Volumenelement durch den Schall

$\vec{F} \quad$ Kraft

$\vec{F}_{\mathrm{D}} \quad$ Stokesreibung

$M_{\mathrm{a}} \quad$ virtuelle Masse der Blase

$N \quad$ Anzahl der Blasen

$P \quad$ Schalldruckamplitude

$P_{\mathrm{B}} \quad$ Blake-Schwelle

$R \quad$ Blasenradius

$\dot{R} \quad$ Geschwindigkeit der Blasenwand

$\ddot{R} \quad$ Beschleunigung der Blasenwand

$R_{0} \quad$ Ruheradius der Blase

$R_{\mathrm{B}} \quad$ Blake-Radius

$R_{\mathrm{n}} \quad$ Gleichgewichtsradius der Blase

$R_{\mathrm{r}} \quad$ Resonanzradius der Blase

Re Reynolds-Zahl

$T \quad$ Schwingungsperiode

$\vec{U} \quad$ Geschwindigkeit der Blase relativ zur Flüssigkeit 
$U_{0} \quad$ akustische Strömungsgeschwindigkeit in der Mitte des Schallstrahls nach Eckart

$U_{\mathrm{el}} \quad$ elektrische Spannung

$V \quad$ Blasenvolumen

$V_{0} \quad$ Ruhevolumen der Blase

$\dot{V}_{i} \quad$ Volumenoszillation von Blase i

$\vec{X} \quad$ Position des Blasenmittelpunktes

$\dot{\vec{X}} \quad$ Geschwindigkeit des Blasenmittelpunktes

c Schallgeschwindigkeit

$\vec{f} \quad$ Kraft auf Volumenelement

$k \quad$ Wellenzahl

$k_{\mathrm{r}} \quad$ Realteil der Wellenzahl

$p \quad$ Druck

$p_{0} \quad$ statischer Druck

$p_{1} \quad$ anregender Schalldruck

$p_{2} \quad$ zeitunabhängige Druckkomponente

$p_{\mathrm{A}} \quad$ Anregungsdruck

$p_{\mathrm{g}} \quad$ Gasdruck in der Blase

$p_{\mathrm{i}} \quad$ Innendruck der Blase

$r_{0} \quad$ Radius der flüssigkeitsgefüllten Röhre

$r_{1} \quad$ Radius des scharf begrenzten Schallstrahls

$\vec{r}_{1,2} \quad$ Abstand zwischen Blase 1 und Blase 2

$t \quad$ Zeit

$\vec{u} \quad$ Geschwindigkeit eines Fluidelementes

$\vec{u}_{0} \quad$ ungestörtes Geschwindigkeitsfeld 
$\vec{u}_{1} \quad$ Schallschnelle durch anregenden Schalldruck

$\vec{u}_{2} \quad$ zeitunabhängige Strömungskomponente - akustische Strömung

$\vec{u}_{1 \mathrm{a}} \quad$ rotationsfreie Schallschnelle im freien Medium

$\vec{u}_{1 \mathrm{~b}} \quad$ inkompressible Schallschnelle nahe einer Grenzschicht

$\vec{v} \quad$ Geschwindigkeitsfeld

$\Pi_{\mathrm{r}} \quad$ durch Schallabstrahlung der Blase von der Blase dissipierte Energie

$\Pi_{\text {th }} \quad$ durch thermische Effekte von der Blase dissipierte Energie

$\Pi_{\mathrm{v}} \quad$ durch viskose Effekte von der Blase dissipierte Energie

$\alpha \quad$ Dämpfungskoeffizient

$\alpha_{0} \quad$ linearer Dämpfungkskoeffizient

$\alpha_{\mathrm{nl}} \quad$ nichtlinearer Dämpfungskoeffizient

$\beta \quad$ Blasenvolumen im zweiphasigen Medium

$\kappa \quad$ Polytropenexponent

$\mu^{\prime} \quad$ Volumenviskosität

$\mu \quad$ Scherviskosität

$\omega \quad$ Kreisfrequenz

$\omega_{0} \quad$ Resonanzfrequenz der Blase

$\omega_{\mathrm{A}} \quad$ Kreisfrequenz des anregenden Schallfelds

$\phi \quad$ Phasenwinkel

$\rho \quad$ Dichte

$\rho_{0} \quad$ statische Dichte

$\rho_{1} \quad$ Dichteschwankung durch anregenden Schalldruck

$\rho_{2} \quad$ zeitunabhängige Dichtekomponente

$\sigma \quad$ Oberflächenspannung 
$\tau \quad$ Schalllaufzeit

$\vartheta \quad$ Dämpfungsfaktor

$\langle\ldots\rangle$ Zeitmittelwert 


\section{Literaturverzeichnis}

[1] C. ECKART: Vortices and streams caused by sound waves. Physical Review 73(1), 68-76, 1948.

[2] W. L. NyborG: Acoustic streaming. In: W. P. MAson (Herausgeber): Physical Acoustics, Band 2B, Seiten 265-331, New York, 1965. Academic Press.

[3] L. K. Zarembo: Acoustic streaming. In: L. D. RozenberG (Herausgeber): High-Intensity Ultrasonic Fields, Seiten 135-199, New York, 1971. Plenum Press.

[4] J. Lighthill: Acoustic streaming. Journal of Sound and Vibration 61(3), 391-418, 1978.

[5] M. O. Lamminen, H. W. Walker und L. K. Weavers: Mechanisms and factors influencing the ultrasonic cleaning of particle-fouled ceramic membranes. Journal of Membrane Science 237(1), 213-223, 2004.

[6] G. W. Gale und A. A. Busnaina: Roles of cavitation and acoustic streaming in megasonic cleaning. Particulate Science and Technology 17(3), 229-238, 1999.

[7] A. A. Busnaina und F. DAI: The removal of submicron particles in liquid-based cleaning. Journal of Adhesion 67(1-4), 181-193, 1998.

[8] O. Dahlem, J. Reisse und V. Halloin: The radially vibrating horn: A scaling-up possibility for sonochemical reactions. Chemical Engineering Science 54(13), 2829-2838, 1999.

[9] J. C. Rife, M. I. Bell, J. S. Horwitz, M. N. Kabler, R. C. Y. AuyEUnG und W. J. KIM: Miniature valveless ultrasonic pumps and mixers. Sensors and Actuators A: Physical 86(1-2), 135-140, 2000. 
[10] F. G. Blake: The onset of cavitation in liquids. I. Cavitation threshold sound pressures in water as a function of temperature and hydrostatic pressure. Technischer Bericht 12, Harvard University, Acoustic Research Laboratory, 1949.

[11] W. LaUterborn (Herausgeber): Cavitation and Inhomogenities in Underwater Acoustics, Proceedings of the First International Conference 1979, Göttingen, Berlin, 1980. Springer Verlag.

[12] F. R. Young: Cavitation. McGraw-Hill Book Company, London, 1989.

[13] T. Nowak und R. MetTin: Unsteady translation and repetitive jetting of acoustic cavitation bubbles. submitted to Physical Review Letters.

[14] S. W. DÄHNkE und F. J. KeIL: Modeling of linear pressure fields in sonochemical reactors considering an inhomogeneous density distribution of cavitation bubbles. Chemical Engineering Science 54(13), 2865-2872, 1999.

[15] R. JAMShIDI und G. BREnNER: Dissipation of ultrasonic wave propagation in bubbly liquids considering the effect of compressibility to the first order of acoustical Mach number. Ultrasonics 53(4), 842-848, 2013.

[16] J. KolB und W. L. NyborG: Small-scale acoustic streaming in liquids. Journal of the Acoustical Society of America 28, 1237-1242, 1956.

[17] P. Tho, R. Manasseh und A. Ooi: Cavitation microstreaming patterns in single and multiple bubble systems. Journal of Fluid Mechanics 576(1), 191-233, 2007.

[18] A. A. Doinikov und A. BouAKaz: Acoustic microstreaming around a gas bubble. Journal of the Acoustical Society of America 127(2), 703-709, 2010.

[19] J. R. Blake, G. S. Keen, R. P. Tong und M. Wilson: Acoustic cavitation: the fluid dynamics of non-spherical bubbles. Philosophical Transaction of the Royal Society of London. Series A: Mathematical, Physical and Engineering Sciences 357(1751), 251-267, 1999.

[20] T. NowAK: Experimentelle Untersuchung von Kavitationsstrukturen an Sonotroden. Diplomarbeit, Drittes Physikalisches Institut der Georg-AugustUniversität Göttingen, 2008.

[21] A. Brotchie, F. Grieser und M. Ashokkumar: Characterization of acoustic cavitation bubbles in different sound fields. Journal of Physical Chemistry B 114(34), 11010-11016, 2010. 
[22] O. Philip, W. D. Schmidl und Y. A. Hassan: Development of a high speed particle image velocimetry technique using fluorescent tracers to study steam bubble collapse. Nuclear Engineering and Design 149, 375-385, 1994.

[23] R. J. Adrian und J. Westerweel: Particle Image Velocimetry, Band 30 der Reihe Cambridge Aerospace Series. Cambridge University Press, New York, 2010.

[24] A. Mandroyan, R. Vinnet, Y. Bailly, M.-L. Doche und J.-Y. Hihn: Modification of ultrasound induced activity by the presence of an electrode in a sonoreactor working at two low frequencies (20 and $40 \mathrm{kHz}$ ). Part I: Active zone visualization by laser tomography. Ultrasonics Sonochemistry 16(1), 88-96, 2009.

[25] A. Mandroyan, M.-L. Doche, J.-Y. Hihn, R. Vinnet, Y. Bailly und L. Simonin: Modification of the ultrasound induced activity by the presence of an electrode in a sono-reactor working at low frequencies (20 and $40 \mathrm{kHz})$. Part II: Mapping flow velocities by particle image velocimetry (PIV). Ultrasonics Sonochemistry 16(1), 97-104, 2009.

[26] R. H. NiLson und S. K. GRIFfiths: Enhanced transport by acoustic streaming in deep trench-like cavities. Journal of the Electrochemical Society 149(4), G286-G296, 2002.

[27] J. A. Jensen, P. Pocwiardowski, P. O. Persson, L. Hultman und P. MøLLER: Acoustic streaming enhanced electrodeposition of nickel. Chemical Physics Letters 368(5), 732-737, 2003.

[28] K. J. Park, M. J. Kim, T. Lim, H.-C. KoO und J. J. Kim: Conformal CU seed layer formation by electroless deposition in non-Bosch through Silicon vias. Electrochemical Solid-State Letters 15(5), D26-D28, 2012.

[29] Q. QI und G. J. Brereton: Mechanisms of removal of micron-sized particles by high-frequency ultrasonic waves. Ultrasonics, Ferroelectrics and Frequency Control, IEEE Transactions on 42(4), 619-629, 1995.

[30] J. FRIEND und L. Y. YEO: Microscale acoustofluidics: Microfluidics driven via acoustics and ultrasonics. Reviews of Modern Physics 83(2), 647-704, 2011. 
[31] Z. Guttenberg, A. Rathgeber, S. Keller, J. O. RÄdler, A. WiXForth, M. Kostur, M. Schindler und P. TAlkner: Flow profiling of a surface-acoustic-wave nanopump. Physical Review E 70(5), 056311 (1-12), 2004.

[32] T. Franke, A. R. Abate, D. A. Weitz und A. Wixforth: Surface acoustic wave $(S A W)$ directed droplet flow in microfluidics for PDMS devices. Lab on a Chip 9(18), 2625-2627, 2009.

[33] L. Y. YEO und J. R. FRIEnd: Ultrafast microfluidics using surface acoustic waves. Biomicrofluidics 3, 012002 (1-23), 2009.

[34] K. D. Frampton, S. E. Martin und K. Minor: The scaling of acoustic streaming for application in micro-fluidic devices. Applied Acoustics 64(7), 681-692, 2003.

[35] L. Johansson, J. Enlund, S. Johansson, I. Katardjiev, M. Wiklund und V. YAntCheV: Surface acoustic wave-induced precise particle manipulation in a trapezoidal glass microfluidic channel. Journal of Micromechanics and Microengineering 22(2), 025018 (1-11), 2012.

[36] M. K. Tan, R. Tueung, H. Ervin, L. Y. Yeo und J. Friend: Double aperture focusing transducer for controlling microparticle motion in trapezoidal microchannel with surface acoustic waves. Applied Physics Letters 95(13), 134101 (1-3), 2009

[37] L. Johansson, J. Enlund, S. Johansson, I. Katardjiev und V. YANTCHEV: Surface acoustic wave induced particle manipulation in a PDMS channel - principle concepts for continuous flow applications. Biomedical Microdevices 14(2), 279-289, 2012.

[38] T. Frommelt, M. Kostur, M. Wenzel-Schäfer, P. Talkner, P. HANGG und A. Wixforth: Microfluidic mixing via acoustically driven chaotic advection. Physical Review Letters 100(3), 034502 (1-4), 2008.

[39] K. Sritharan, C. J. Strobl, M. F. Schneider, A. Wixforth und Z. GuttenberG: Acoustic mixing at low Reynold's numbers. Applied Physics Letters 88(5), 054102 (1-3), 2006.

[40] G. J. Brereton. und B. A. Bruno: Particle removal by focused ultrasound. Journal of Sound and Vibration 173(5), 683-698, 1994. 
[41] D. G. Seiler und C. K. Campbell: Fabrication of thin film interdigital transducers for surface acoustic wave devices operating at gigahertz frequencies. Thin Solid Films 62(1), 109-114, 1979.

[42] J. Estevadeordal und L. Goss: PIV with LED: particle shadow velocimetry (PSV). In: 43rd AIAA Aerospace Sciences Meeting and Exhibit, Meeting Papers, Seiten 12355-12364, 2005.

[43] C. G. Zoski (Herausgeber): Handbook of Electrochemistry. Elsevier Science Publishers Ltd., Oxford, 2007.

[44] Comsol, Burlington, MA 01803 USA: Comsol Multiphysics, Version 4.3a, 2012. Finite Elemente Simulations Software.

[45] Lord RaYleigh: Theory of Sound. Dover, New York, 1896.

[46] H. Schlichting: Boundary Layer Theory. McGraw-Hill, New York, 1955.

[47] L. N. Liebermann: The second viscosity of liquids. Physical Review 75(9), 1415-1422, 1949.

[48] D. H. Trevena: Cavitation and Tension in Liquids. Adam Hilger, Bristol, 1987.

[49] W. Lauterborn: Cavitation. In: Encyclopedia of Acoustics, Band 1, Seiten 263-270, Hoboken, 1997. John Wiley \& Sons.

[50] D. Obreschkow, P. Kobel, N. Dorsaz, A. de Bosset, C. NicolLIER und M. FARHAT: Cavitation bubble dynamics inside liquid drops in microgravity. Physical Review Letters 97(9), 094502 (1-4), 2006.

[51] W. Lauterborn: Erzeugung und Hochfrequenzkinematographie von Hohlräumen in Wasser mit einem Rubin-Laser. Research Film 7, 25-31, 1970.

[52] W. Lauterborn: Kavitation durch Laserlicht. Acustica 31, 51-78, 1974.

[53] D. Silberrad: Propeller erosion. Engineering 93, 33-35, 1912.

[54] T. G. Leighton: The Acoustic Bubble. Academic Press, London, 1994.

[55] C. E. Brennen: Cavitation and Bubble Dynamics, Band 44 der Reihe Oxford Engineering Science. Oxford University Press, New York, 1995. 
[56] A. Lippert, P. Engesser, G. Ferrell, J. Klitzke, M. Köffler, F. Kumnig, J. Leberzammer, R. Obweger, A. Pfeuffer, H. Sax und H. OKORN-SCHMIDT: Behaviour of a well-designed megasonic cleaning system. Solid State Phenomena 103-104, 155-158, 2005.

[57] K. Mørch: Reflections on Cavitation Nuclei in Water. Physics of Fluids 19, $072104(1-7), 2006$.

[58] V. F. K. BJerknes: Fields of Force. Columbia University Press, New York, 1906.

[59] V. F. K. BJerknes: Die Kraftfelder. Vieweg und Sohn, Braunschweig, 1909 .

[60] M. MinnaERT: On musical air-bubbles and the sounds of running water. The London, Edinburgh, and Dublin Philosophical Magazine and Journal of Science 16(7), 235-248, 1933.

[61] R. Mettin: From a single bubble to bubble structures in acoustic cavitation. In: T. Kurz, U. PARlitz und U. KAatze (Herausgeber): Oscillations, Waves and Interactions, Seiten 171-198, Göttingen, 2007. Universitätsverlag Göttingen.

[62] O. LouisnARD: A simple model of ultrasound propagation in a cavitating liquid. Part I: Theory, nonlinear attenuation and traveling wave generation. Ultrasonics Sonochemistry 19(1), 56-65, 2012.

[63] O. Louisnard: A simple model of ultrasound propagation in a cavitating liquid. Part II: Primary Bjerknes force and bubble structures. Ultrasonics Sonochemistry 19(1), 66-76, 2012.

[64] C. Campos-Pozuelo, C. Granger, C. Vanhille, A. Moussatov und B. Dubus: Experimental and theoretical investigation of the mean acoustic pressure in the cavitation field. Ultrasonics Sonochemistry 12(1-2), 79-84, 2005 .

[65] R. Mettin, J.-O. Claussen und C. Campos-Pozuelo: Sound propagation and bubble motion in a cavitating channel. In: 19th International Congress on Acoustics, Band 38 der Reihe Revista de Acústica, Seiten 1-6, Madrid, 2007. Sociedad Espanola de Acústica (SEA).

[66] P. Koch, D. Krefting, T. Tervo, R. Mettin und W. Lauterborn: Bubble path simulations in standing and traveling acoustic waves. In: Proceedings of ICA 2004, Seiten V3571-V3572, Kyoto, 2004. 
[67] R. Mettin, I. Akhatov, U. Parlitz, C.-D. Ohl und W. Lauterborn: Bjerknes forces between small cavitation bubbles in a strong acoustic field. Physical Review E 56(3), 2924-2931, 1997.

[68] L. D. Landau und E. M. Lifschitz: Hydrodynamik, Band 6 der Reihe Lehrbuch der Theoretischen Physik. Akademie Verlag, Berlin, 5. Auflage, 1991.

[69] R. Mettin und T. Nowak: Experimentelle Beobachtung der sprunghaften Blasenbewegung in Kavitationsfeldern. In: U. JEKOSCH und R. HoffMAnn (Herausgeber): Fortschritte der Akustik - DAGA 2008 Dresden, Seiten 479-480. Deutsche Gesellschaft für Akustik e. V. (DEGA), 2008.

[70] T. Nowak, R. Mettin und W. Lauterborn: Bubble motion and jetting at sonotrodes. In: M. M. Boone und T. U. Delft (Herausgeber): NAG/DAGA 2009, International Conference on Acoustics, Rotterdam, Seiten 712-714, Berlin, 2009. Deutsche Gesellschaft für Akustik e. V. (DEGA).

[71] G. K. Batchelor: An Introduction to Fluid Dynamics. Cambridge University Press, New York, 2000.

[72] V. G. Levich: Physicochemical Hydrodynamics. Prentice-Hall Inc., Englewood Cliffs, 1962.

[73] L. Wissmann: Laborbericht 200\%. Drittes Physikalisches Institut der Georg-August-Universität Göttingen, unveröffentlicht.

[74] O. M. LoRD RAyleigh: On the pressure developed in a liquid during the collapse of a spherical cavity. The London, Edinburgh, and Dublin Philosophical Magazine and Journal of Science 34(200), 94-98, 1917.

[75] M. S. Plesset: The dynamics of cavitation bubbles. Journal of Applied Mechanics 16, 277-282, 1949.

[76] B. E. Noltingk und E. A. Neppiras: Cavitation produced by ultrasonics. Proceedings of the Physical Society Section B 63(9), 674-685, 1950.

[77] E. A. Neppiras und B. E. Noltingk: Cavitation produced by ultrasonics: Theoretical conditions for the onset of cavitation. Proceedings of the Physical Society Section B 64(12), 1032-1038, 1951.

[78] H. PORITSKY: The collapse or growth of a spherical bubble or cavity in a viscous fluid. In: E. STERnBERG (Herausgeber): Proceedings of the first US National Congress on Applied Mechanics, Seiten 813-821, New York, 1952. 
[79] J. B. Keller und M. Miksis: Bubble oscillations of large amplitude. Journal of the Acoustical Society of America 68(2), 628-633, 1980.

[80] A. Pluta: Dynamik wechselwirkender Kavitationsblasen. Diplomarbeit, Drittes Physikalisches Institut der Georg-August-Universität Göttingen, unveröffentlicht.

[81] M. L. Calvisi, O. Lindau, J. R. Blake und A. J. Szeri: Shape stability and violent collapse of microbubbles in acoustic traveling waves. Physics of Fluids 19, 047101 (1-15), 2007.

[82] A. J. Reddy und A. J. Szeri: Coupled dynamics of translation and collapse of acoustically driven microbubbles. Journal of the Acoustical Society of America 112(4), 1346-1352, 2002.

[83] A. J. REDdy und A. J. Szeri: Shape stability of unsteadily translating bubbles. Physics of Fluids 14(7), 2216-2224, 2002.

[84] S. Sakamoto und Y. Watanabe: Effects of existence of micro bubbles for increase of acoustic streaming. Japanese Journal of Applied Physics 38(5B), 3050-3052, 1999.

[85] H. Nomura und T. Kamakura: Acoustic radiation force acting on a small bubble in progressive waves. In: W. LAUTERBORN und T. KurZ (Herausgeber): Nonlinear Acoustics at the Turn of the Millenium, 15th International Symposium on Nonlinear Acoustics, ISNA 15, Göttingen, Germany, Band 524 der Reihe AIP Conference Proceedings, Seiten 363-366, Melville, New York, 2000. American Institute of Physics.

[86] M. S. Plesset und A. Prosperetti: Bubble dynamics and cavitation. Annual Review of Fluid Mechanics 9, 145-185, 1977.

[87] K. W. Commander und A. Prosperetti: Linear pressure waves in bubbly liquids: Comparision between theory and experiments. Journal of the Acoustical Society of America 85(2), 732-746, 1989.

[88] H. Mitome, T. Kozuka, T. Tuziuti und L. Wang: Quasi acoustic streaming induced by generation of cavitation bubbles. In: Ultrasonics Symposium, 1997. Proceedings, $199^{7}$ IEEE, Band 1, Seiten 533-536. IEEE, 1997.

[89] S. A. EldER: Cavitation microstreaming. Journal of the Acoustical Society of America 31(1), 54-64, 1959. 
[90] D. Kröninger, K. Köhler, T. Kurz und W. Lauterborn: Particle tracking velocimetry of the flow field around a collapsing cavitation bubble. Experiments in Fluids 48(3), 395-408, 2010.

[91] A. Smolianski, H. HaArio und P. Luukka: Vortex shedding behind a rising bubble and two-bubble coalescence: A numerical approach. Applied Mathematical Modelling 29, 615-632, 2005.

[92] C. Wang und J. Cheng: Cavitation microstreaming generated by a bubble pair in an ultrasound field. Journal of the Acoustical Society of America 134, 1675-1682, 2013.

[93] T. NowAK und R. MetTin: Acoustic streaming: comparison of cavitating and non-cavitating water. In: AIA-DAGA 2013, Proceedings of the International Conference on Acoustics, Merano, Seiten 481-484. Deutsche Gesellschaft für Akustik e. V. (DEGA), 2013.

[94] C. Willert, B. Stasicki, J. Klinner und S. Moessner: Pulsed operation of high-power light emitting diodes for imaging flow velocimetry. Measurement Science Technology 21(11), 1-12, 2010.

[95] W. Thielicke und E. J. Stamhuis: PIVLab 1.32 - Time-resolved digital particle image velocimetry tool for matlab. pivlab.blogspot.de, 2012.

[96] MathWorks: Matlab, version R2011b, 2011. Matrixorientiertes Programmpaket zur Lösung und Visualisierung mathematischer Probleme.

[97] R. J. AdRIAn: Particle-imaging techniques for experimental fluid mechanics. Annual Review of Fluid Mechanics 23, 261-304, 1991.

[98] E. J. STAmHUIS: Basics and principles of particle image velocimetry (PIV) for mapping biogenic and biologically relevant flows. Aquatic Ecology 40(4), 463-479, 2006.

[99] L. A. CRum: Acoustic cavitation series: Part five Rectified diffusion. Ultrasonics 22(5), 215-223, 1984.

[100] H. KutTrufF: Physik und Technik des Ultraschalls. S. Hirzel Verlag, Stuttgart, 1988.

[101] P. Bradshaw: An Introduction to Turbulence and its Measurement. Pergamon Press, Oxford, 1971. 
[102] Y. D. Afanas'ev und S. I. Voropaev: Horizontal submerged jet in a stratified fluid. Fluid dynamics 28(6), 757-762, 1993.

[103] H. NyQUiST: Certain topics in telegraph transmission theory. Transaction of the American Institute of Electrical Engineers 47, 617-644, 1928.

[104] A. Tokuhiro, M. Maekawa, K. Iizuka, K. Hishida und M. Maeda: Turbulent flow past a bubble and an ellipsoid using shadow-image and PIV techniques. International Journal of Multiphase Flow 24, 1383-1406, 1998.

[105] S. Hosokawa und A. Tomiyama: Bubble-induced pseudo turbulence in laminar pipe flows. International Journal of Heat and Fluid Flow 40, 97-105, 2013.

[106] T. Nowak., A. Thiemann, F. Holsteyns, A. Lippert und R. MetTIN: Messung der akustisch induzierten Strömungen mit und ohne Kavitation. In: J. Becker-SchweITzer und G. NotBohm (Herausgeber): Fortschritte der Akustik - DAGA 2011 Düsseldorf, Seiten 923-924, Berlin, 2011. Deutsche Gesellschaft für Akustik e. V. (DEGA).

[107] T. Nowak, C. Cairos, F. Holsteyns, A. Lippert und R. Mettin: Acoustic streaming at gigasonic piston-like transducers. In: H. HANSELKA (Herausgeber): Fortschritte der Akustik - DAGA 2012 Darmstadt, Seiten 457-458, Berlin, 2012. Deutsche Gesellschaft für Akustik e. V. (DEGA).

[108] H. Okorn-Schmidt, F. Holsteyns, A. Lippert, D. Mui, M. KawaguChi, C. Lechner, P. Frommhold, T. Nowak, F. Reuter, M. Banchs i Piqué, C. Cairós und R. Mettin: Particle cleaning technologies to meet advanced semiconductor device process requirements. submitted to ECS Journal of Solid State Science and Technology.

[109] F. Kohlrausch: Praktische Physik: zum Gebrauch für Unterricht, Forschung und Technik, Band 3. B. G. Teubner, Stuttgart, 23. Auflage, 1986.

[110] C. Cairos, T. Nowak., F. Reuter und R. Mettin: Studying cavitation and acoustic streaming by electrochemistry. In: H. HANSELKA (Herausgeber): Fortschritte der Akustik - DAGA 2012 Darmstadt, Seiten 453-454, Berlin, 2012. Deutsche Gesellschaft für Akustik e. V. (DEGA).

[111] W. P. Mason: Piezoelectric Crystals and their Application to Ultrasonics, Band 4. D. Van Nostrand Company, Inc., Princeton, 1959. 
[112] D. Sindayihebura, L. Bolle, A. Cornet und L. Johannes: Theoretical and experimental study of transducers aimed at low-frequency ultrasonic atomization of liquids. Journal of the Acoustical Society of America 103(3), 1442-1448, 1998. 



\section{Danksagung}

Bedanken möchte ich mich...

... bei Prof. Dr. Christoph F. Schmidt für die Ermöglichung dieser Arbeit.

... bei Prof. Dr. Werner Lauterborn für die Übernahme des Korreferats und die vielen hilfreichen wissenschaftlichen Diskussionen.

... bei Dr. Robert Mettin für die Betreuung der Arbeit, die tatkräftige Unterstützung bei wissenschaftlichen und organisatorischen Problemen und für die engagierte Leitung der Arbeitsgruppe.

... bei allen Mitgliedern des Christian Doppler Labors für Kavitation und Mikroerosion für die erfolgreiche Zusammenarbeit, die fachlichen und nicht-fachlichen Diskussionen, die schnelle Hilfe bei Problemen und die abwechslungsreichen Fahrten zu Konferenzen und Review-Meetings, dabei besonders bei Philipp Frommhold und Andrea Thiemann.

... bei Alexander Lippert und Dr. Frank Holsteyns für die gute Zusammenarbeit, die interessanten wissenschaftlichen Fragestellungen und die abwechslungsreichen Abende in Göttingen und auf Review-Meetings.

... bei den Mitarbeiterinnen und Mitarbeitern der feinmechanischen Werkstatt und der Elektronikwerkstatt des Dritten Physikalischen Instituts, dabei besonders bei den Leitern Dieter Hille und Dr. Karl Lautscham, außerdem bei Thomas Geiling für die engagierte Hilfe bei IT-Problemen aller Art.

... bei den Sekretärinnen Elke Zech, Nicole Rehbein, Sabine Huhnold und Ursula Hahn-Wörgötter für die hilfreiche Unterstützung bei formalen Dingen.

... bei allen Mitarbeiterinnen und Mitarbeitern des Dritten Physikalischen Institutes für die Hilfe bei Software-Problemen und chemischen und optischen Fragestellungen, dabei besonders bei Hendrik Söhnholz. 
... bei allen meinen Freundinnen und Freunden für die gemeinsam verbrachte Zeit, dabei besonders bei Maik Schubert für die täglichen Mittagspausen und das Korrekturlesen der Theorie.

... besonders bei meinen Eltern für die Ermöglichung des Studiums, die Unterstützung und das Korrekturlesen dieser Arbeit.

... besonders bei Anissa für die Unterstützung, die Geduld in stressigen Phasen und das Korrekturlesen dieser Arbeit.

... bei der Christian Doppler Forschungsgesellschaft und der Lam Research AG für die Finanzierung dieser Arbeit. 

